
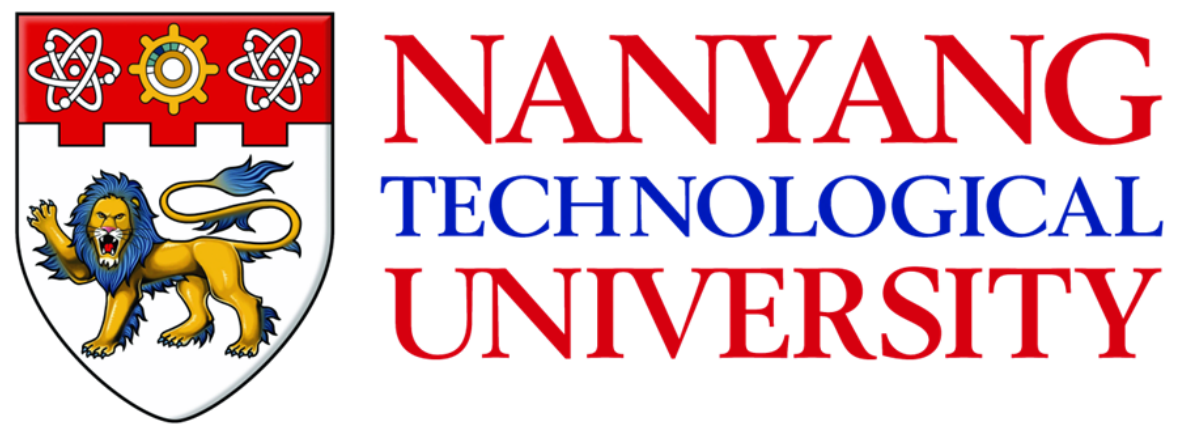

\title{
A MOLECULAR DISSECTION OF THE RUBISCO ACTIVATION SYSTEM FROM RICE
}

DEVENDRA SHIVHARE

SCHOOL OF BIOLOGICAL SCIENCES 



\section{A MOLECULAR DISSECTION OF THE RUBISCO ACTIVATION SYSTEM FROM RICE}

\section{DEVENDRA SHIVHARE}

\section{School of Biological Sciences}

A thesis submitted to the Nanyang Technological University in partial fulfillment of the requirement for the degree of Doctor of Philosophy 



\section{Acknowledgments}

First and foremost, I would like to express my profound gratitude to my supervisor, Asst Prof. Oliver Mueller-Cajar, for his continuous encouragement and expert guidance. His expert knowledge and never ending passion for science has inspired me throughout this journey. His constant support and patience has helped me complete this thesis successfully. I am thankful to Prof. Daniela Rhodes and Asst Prof. Guillaume Thibault for being my thesis advisory committee. Their critical inputs have helped me frame a meaningful thesis.

I am very grateful to Dr. Tsai Yi Chin (Candace) for being an immediate mentor and helping me get started with most of the technical skills. She also creates a fun loving environment that makes research a much more joyful experience. I extend a hearty thanks to Tobi for his intellectual input during my project. Our interesting discussion related to science and personal life has always been a good experience.

Nitin, my lab mate has been much more than a colleague to me. He has been a friend as well as a brother during this phase of $\mathrm{PhD}$. He has helped me in every level of this research including the wrapping up of this thesis. My heartfelt thanks to him for each and every help. I am very thankful to Ramya for extending her help in designing my figures and added a creative touch to it. Having her around has definitely made work in lab a better experience.

My special thanks to Zhijun, Liu Di, Zhen Guo, Jed, Jian Ann, Steve and Lynette for all their help and support during my doctoral research. I would also like to thank all the past members of the lab including all the FYPs and attachment students

I would like to thank Geeta, for helping me edit my thesis. Another special person who arrived to the lab is Alex. He started his journey in our lab around the same time when I started writing my thesis. He sat right next to me and kept giving constant support to finish my thesis. A big thanks for his encouragement and making me believe that I can do it. 
Most importantly I would like to thank my friends, Dhiraj, Akshaya, Arvind and Tofayel for being the family in SBS. Their emotional support and love has definitely made my stay, a pleasant one here.

I would love to extend my deepest gratitude to my loving family for their encouragement and support. In no words, I can express my gratitude towards my mother. It's her believe, patience and prayers that I am able to finish this thesis.

I dedicate this thesis to the most important person of my life, my late father, who always trusted my ability and gave me all the encouragements and strength throughout my life. It is his blessing that today I am able to fulfil his dream.

I would like to thank my better half, Reeti for her love, trust, encouragement and my in-laws for their support and patience.

I gratefully acknowledge the financial support rendered by the Nanyang Technological University of Singapore in the form of Research Scholarship and its excellent research facility.

Finally, I am grateful to almighty god for all his blessings. 


\section{Table of contents}

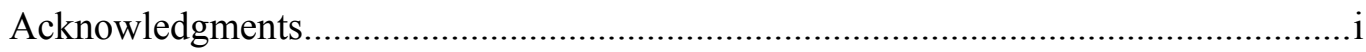

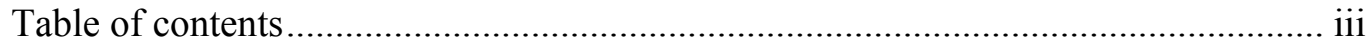

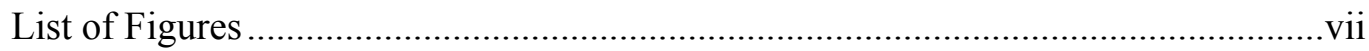

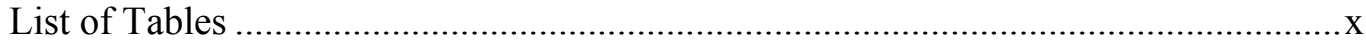

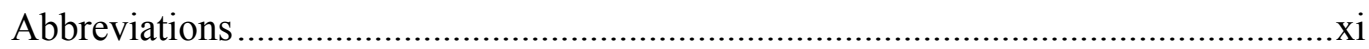

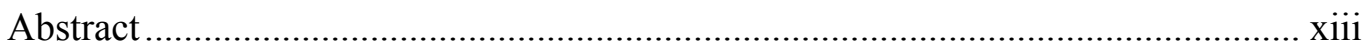

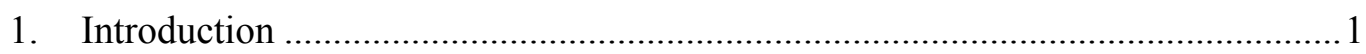

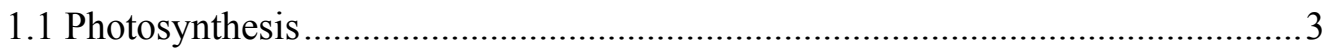

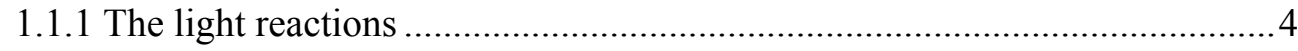

1.1.2 The dark reactions (The Calvin-Benson cycle) .......................................... 6

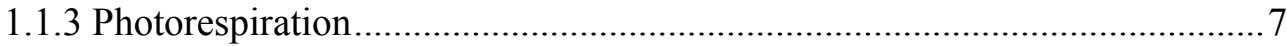

1.1.4 Carbon concentrating mechanisms ......................................................... 9

1.2 Ribulose 1, 5- bisphosphate carboxylase/oxygenase (Rubisco)....................... 10

1.2.1 The structure and classification of Rubisco................................................ 11

1.2.2 Reaction mechanism: carbamylation and catalysis .................................13

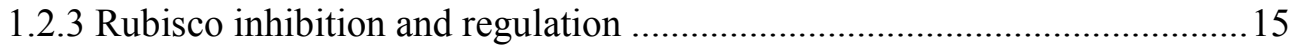

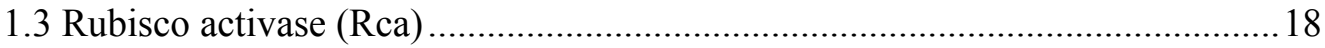

1.3.1 Rubisco activases are members of the AAA + family of proteins .............. 19

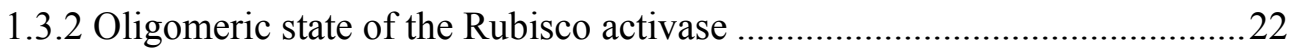

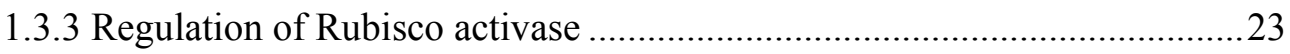

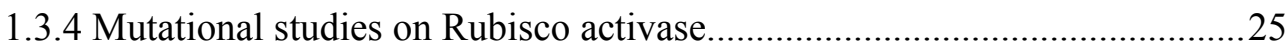

1.3.5 The role of Rubisco activase in regulating photosynthesis at elevated

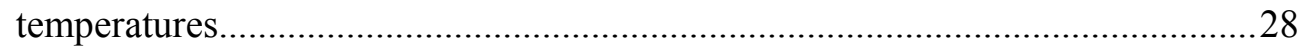

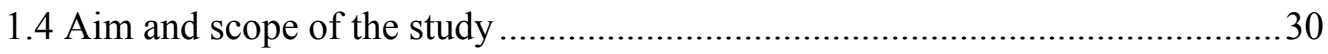

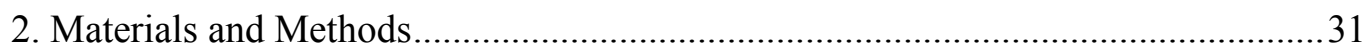

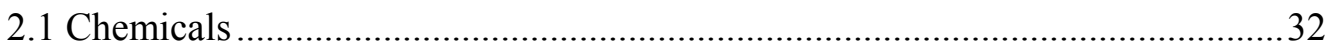

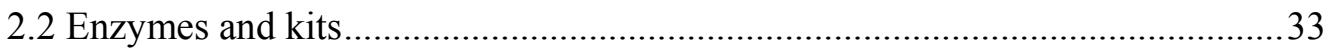

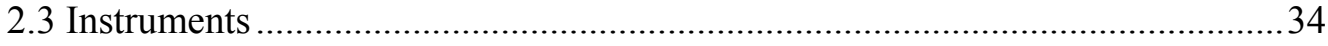

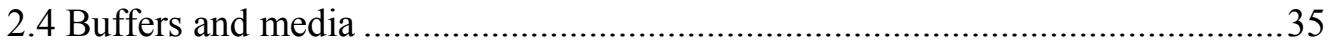

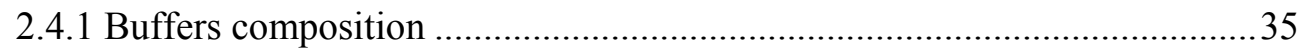

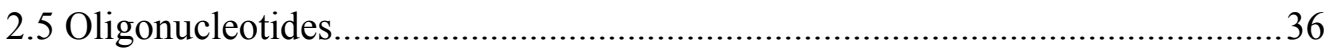

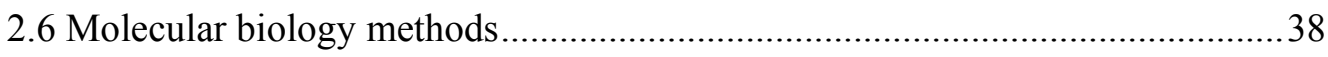




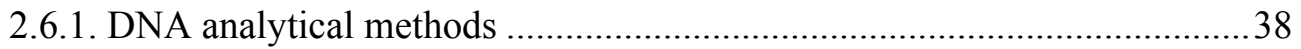

2.6.2 Competent $E$. coli cells preparation and transformation ............................39

2.6.3 Plasmid and DNA-fragment purification ................................................. 40

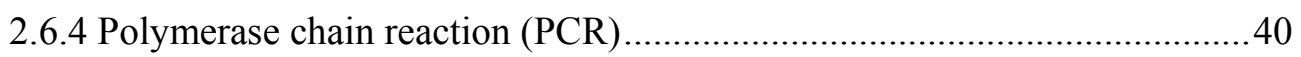

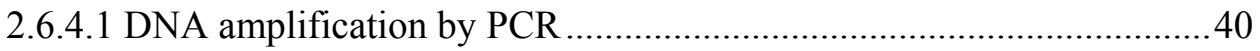

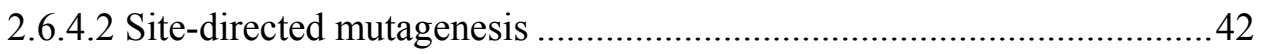

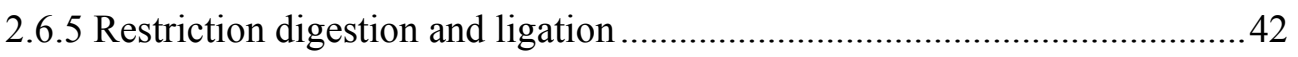

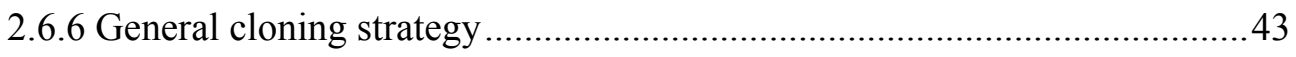

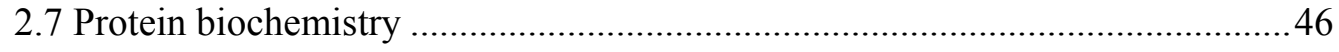

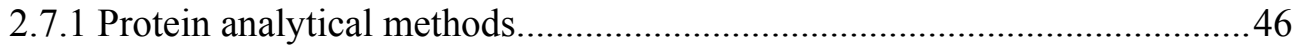

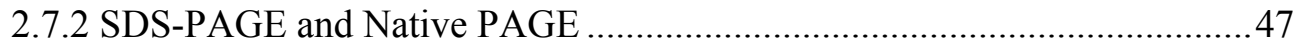

2.7.3 Coomassie blue staining of SDS and native PAGE .................................. 48

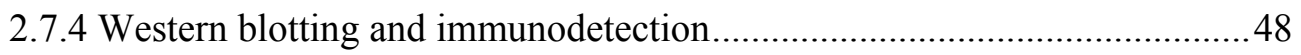

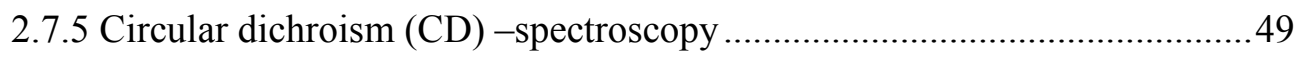

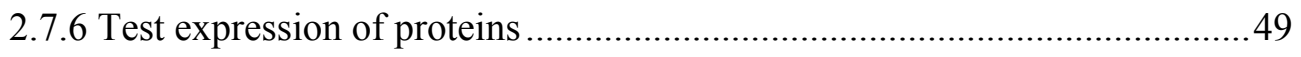

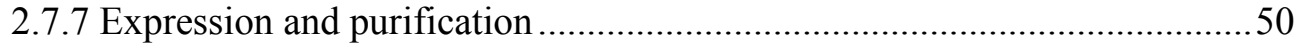

2.7.7.1 Purification of $\mathrm{His}_{6} \mathrm{Ub}$-fusion constructs .........................................5

2.7.8 Purification of Rubisco from the leaves of Oryza sativa...........................51

2.7.9 Analytical size exclusion chromatograpghy ...........................................52

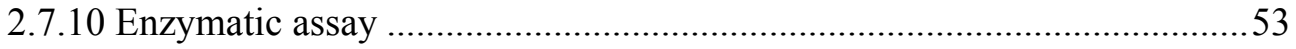

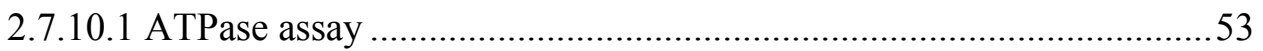

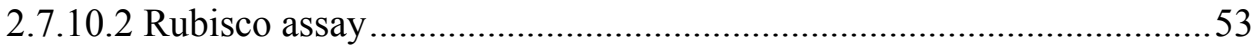

2.7.10.3. Variations of enzymatic assay ....................................................5 54

3. Biochemical characterization of the Rubisco activation system of the staple crop

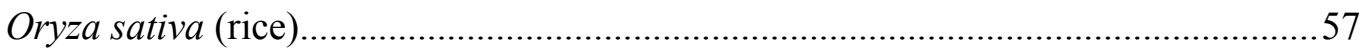

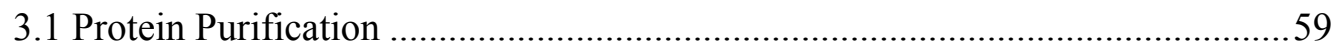

3.1.1 Purification of Oryza sativa Rubisco activase (Rca) isoforms...................59

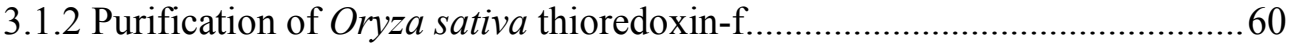

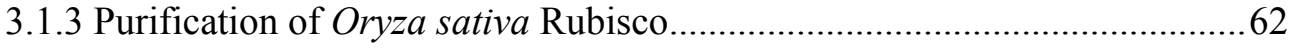

3.2 Recombinant Rca isoforms were biochemically active and can activate

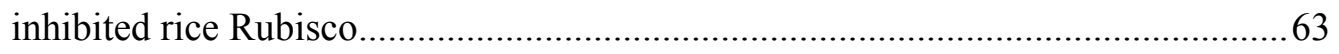

3.3 Redox regulation of the OsRca $\alpha$ in the presence of thioredoxin-f.....................66 
3.3.1 Evidence for covalent complex formation between OsRca $\alpha$ and thioredoxin-f

3.4 Characterization of Rubisco activase thermostability

3.4.1 Rice Rubisco activases are also relatively thermolabile.

3.5 Subunit mixing with OsRca $\alpha$ reduces the ATPase activity of the OsRca $\beta \ldots \ldots . . .74$

3.5.1 Characterization of the ATPase inactive OsRca mutants. .76

3.5.2 Further evidence for hetero-oligomerization between Rca isoforms .78

3.6 Subunit mixing and redox state does not affect the thermotolerance of the rice activase

4. Characterization of a thermostable Rubisco activase from Agave tequilana 83

4.1 Purification of Agave tequilana Rca isoforms .85

4.2 Both agave isoforms were biochemically active and can activate inhibited rice

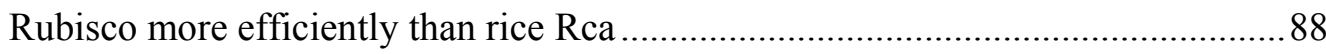

4.3 Agave Rca isoforms are highly thermostable ..... 90

4.4 Rice and agave activase subunits form hetero-oligomers displaying intermediate functionality but subunit-specific thermostability. 92

4.5 Characterization of chimeric mutants localized thermostability and high activase activity to the nucleotide binding domain (NBD) of AtRca $\beta$ 95

4.6 ATPase activity of AtRca isoform was not enhanced in presence of DTT and thioredoxin- $f$ of rice

4.7 Mutational analysis of various residues at NBD revealed that a single glutamate (217) to glutamine (E-Q) substitution confers high in vitro activase activity to OsRca $\beta$

4.8 Mutational analysis of the newly identified surface loop residues revealed a key lysine (216) important for Rubisco-Rca interaction.

5. Generation of a rice Rubisco activase molecular toolbox and towards an improved mechanistic appreciation. 106

5.1 Subunit doping experiment with Walker B mutants indicates strong cooperativity between subunits

5.2 Validating the phenotype of key OsRca variants, studied previously in other plant Rca systems

5.2.1 Rubisco interaction site mutants display variable levels of importance in context of oligomer function 113

5.3 Structure based identification of novel Rubisco interacting residues 119

5.3.1 The key pore loop 1 mutant (A143V) reveals strong subunit coupling with respect to Rubisco activase activity 
5.4 Mutational analysis of phophorylating residue at the NTD indicates its role in Rubisco-Rca interaction and might be an alternate regulatory mechanism in plants under light dark transition

5.5 Mutation of conserved arginine (R293V) at the Rca subunit interface ablates its

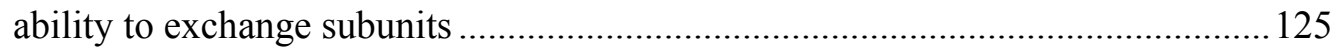

6. A preliminary characterization of the putative Rubisco activase isoform (Rca2) of rice......

6.1 Purification of OsRca2

$6.2 \Delta \mathrm{N} 21$ OsRca2 migrates as a monodisperse species by gel filtration and native PAGE

$6.3 \Delta \mathrm{N} 210$ sRca2 was ATPase inactive and unable to activate rice Rubisco but forms ATPase functional hetero-oligomers with OsRca $\beta$.

6.4 Demonstration of a novel complex between $\Delta \mathrm{N} 21$ OsRca2 and OsRca $\beta$ when

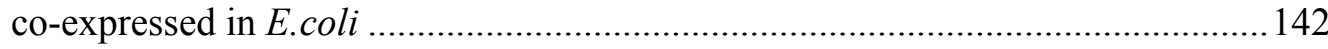

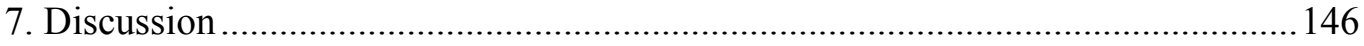

7.1 Rice activase isoforms exhibit typical regulatory behaviour and have similar thermostability to other characterized homologues .............................................. 147

7.2 Subunit exchange and cooperativity between Rca isoforms ........................... 149

7.3 Comparison between biochemical and thermal properties of rice (C3) and agave (CAM)

7.4 Insight into thermostability and Rubisco activase mechanism gleaned through

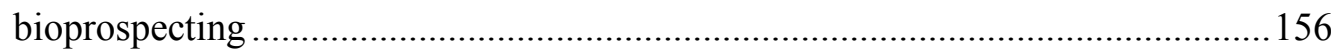

7.5 New insights into the Rubisco activation mechanism...................................157

7.6 Biochemical insights into the regulation of Rca by phosphorylation ...............163

7.7 Distant classes of Rubisco activase have convergently evolved to remodel their

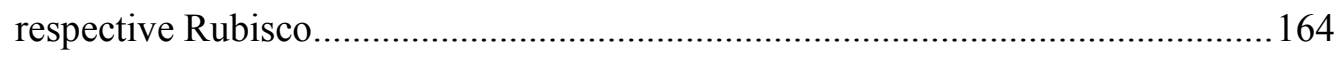

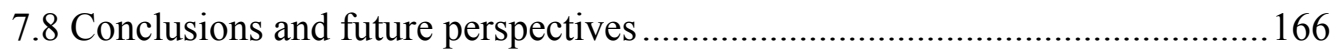

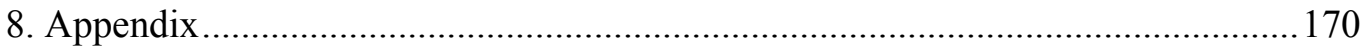

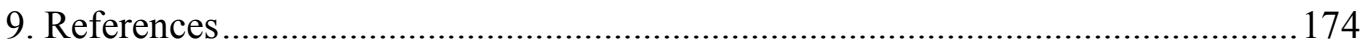




\section{List of Figures}

Fig. 1.1: Schematic illustration of the basic process of photosynthesis that takes place in the chloroplast......

Fig. 1.2: Light dependent reactions of photosynthesis...............................................5

Fig. 1.3: The Calvin-Benson cycle.................................................................

Fig. 1.4: Schematic overview of the photorespiratory pathway................................

Fig. 1.5: Representatives of the four forms of Rubisco.............................................11

Fig. 1.6: The carboxylation and oxygenation reaction catalyzed by Rubisco.............14

Fig. 1.7: Regulation of Rubisco by Rubisco activase.................................................16

Fig. 1.8: Structure and domain architecture of Rca from Nicotiana tabacum.............21

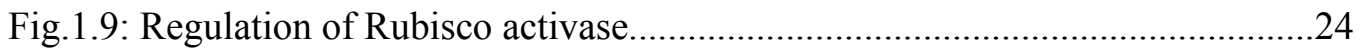

Fig. 1.10: Domain architecture of Rubisco activase with a bird eye view of mutational

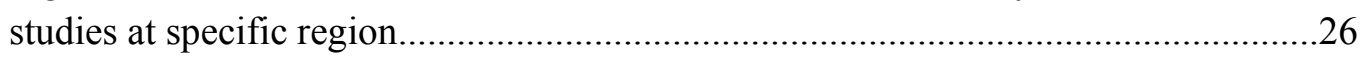

Fig. 1.11: Activase based model of thermal inhibition of photosynthesis at high

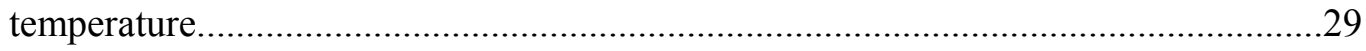

Fig. 2.1: Schematic representation of the generation of chimeric mutants..................46

Fig. 2.2: Representation of the reactions that occurred during an ATPase activity

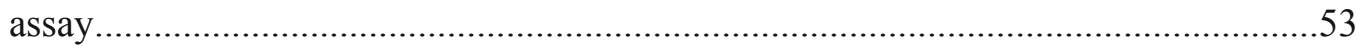

Fig. 2.3: Representation of reactions that occurred during a Rubisco activity

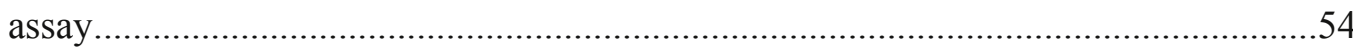

Fig. 3.1: Representative purification of OsRca $\alpha$ and OsRcaß...................................59

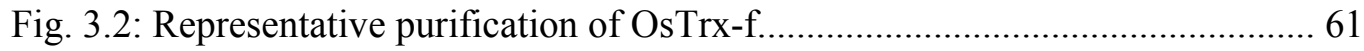

Fig. 3.3: Representative purification of Rubisco................................................... 63

Fig. 3.4: Biochemical properties of Rubisco activase isoforms from Oryza sativa. .66

Fig. 3.5: ATPase activity of OsRca $\alpha$ was stimulated in presence of DTT and thioredoxin $-\mathrm{f}$

Fig. 3.6: SDS-PAGE analysis of the reaction mixtures containing OsRca $\alpha$ and Trx-

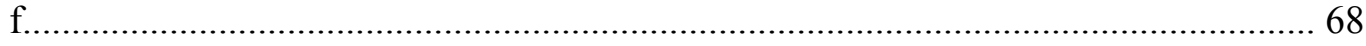

Fig. 3.7: Decline in ATPase Activity of Rubisco activase upon heat treatment......... 70 
Fig. 3.8: CD spectroscopic profile of Rubisco activases at different temperature........72

Fig. 3.9: Thermostability analysis of Rubisco activase ...........................................73

Fig. 3.10: Subunit mixing with OsRca $\alpha$ reduces the ATPase activity of OsRca $\beta \ldots . . . .75$

Fig. 3.11: Mutations at the Walker A and Walker B motifs of Rubisco activase abolish

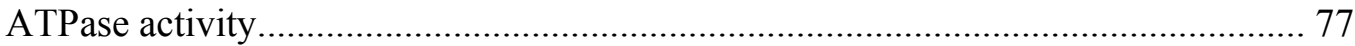

Fig. 3.12: Subunit interaction study using analytical gel filtration and SDS-PAGE...79

Fig. 3.13: Effect of subunit mixing and Thioredoxin-f on thermostability..................82

Fig. 4.1: Schematic diagram of a day night cycle in a typical CAM plant..................85

Fig. 4.2: Sequeunce alignment of Oryza sativa and Agave tequilana activase

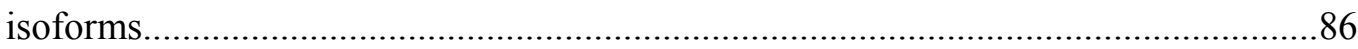

Fig. 4.3: Representative purification of AtRca $\alpha$ and AtRcaß ................................ 87

Fig. 4.4: Biochemical properties of Rubisco activase isoforms from Oryza sativa and

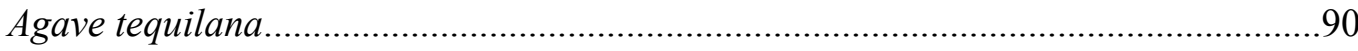

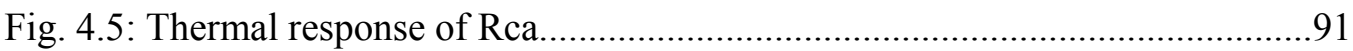

Fig. 4.6: Inter-species subunit interaction between the Agave and the rice

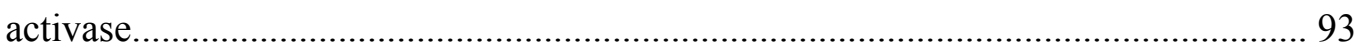

Fig. 4.7: Thermal and biochemical characterization of chimeric mutants................. 95

Fig. 4.8: Comparative effect of rice thioredoxin-f on ATPase activity of AtRca $\ldots . . . . .99$

Fig. 4.9: Thermal and biochemical characterization of site-directed point mutants in

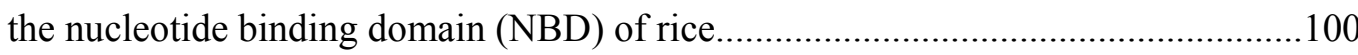

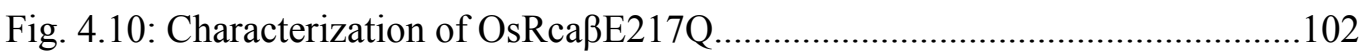

Fig. 4.11: Characterization of the newly identified surface loop residues................ 104

Fig. 5.1: Theoretical model to predict the effect of subunit doping..........................109

Fig. 5.2: Titration of WT $\beta$-Rca with increasing concentration of ATPase inactive

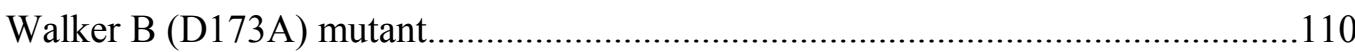

Fig. 5.3: Characterization of Rubisco activase mutants....................................... 113

Fig. 5.4: Titration of WT $\beta$-Rca with increasing concentration of interaction site

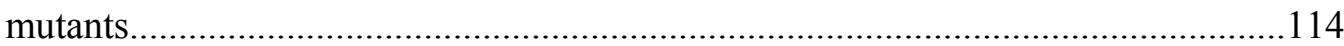

Fig. 5.5: Titration of $\Delta \mathrm{N} 67$ Rca with increasing concentrations of different mutants. 
Fig. 5.6: Mutational analysis of pore loop 1, its extension and surface residues.......120

Fig. 5.7: Titration of WT $\beta$-Rca with increasing concentrations of pore loop 1 mutant (A143V).

Fig. 5.8: Mutation of the phosphorylated residue at the N-terminal domain of rice

Rca... 125

Fig. 5.9: Mutation of conserved arginine at Rca interface.

Fig. 6.1: Phylogenetic tree of plant and algal activase and putative Rca2 sequences 130

Fig. 6.2: Protein sequence alignment between plant Rca and Rca2 isoforms 132

Fig. 6.3: Representative purification of $\Delta \mathrm{N} 21 \mathrm{OsR}$ ca2 134

Fig. 6.4: Rca2 eluted as monodispersed species as analyzed by analytical gel filtration chromatography and native PAGE 135

Fig. 6.5: Biochemical characterization of $\Delta \mathrm{N} 21$ OsRca2. 137

Fig. 6.6: Biochemical characterization of OsRca2..... 138

Fig. 6.7: Hypothesized scheme for the formation of functional hetero-oligomer between OsRca2 and OsRcaßD173A.....

Fig. 6.8: Co-expression studies between $\Delta \mathrm{N} 21$ OsRca2 and Rca $\beta$. 143

Fig. 6.9: Complex formation between $\Delta \mathrm{N} 210$ sRca 2 and Rca $\beta$ during coexpression. 144

Fig. 7.1: Protein sequence alignment of Rubisco activase from selected species.... 159

Fig. 7.2: Mechanistic model for Rubisco activation in plant...... 162

Fig. 7.3: Mechanism of Rubisco activation in different system. 165

Fig. 8.1: Tissue specific expression level of Rca2 and Rubisco activase as obtained from Arabidopsis eFP browser. 


\section{List of Tables}

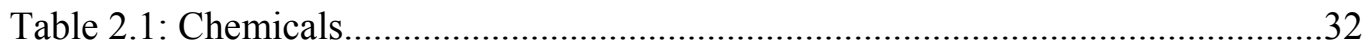

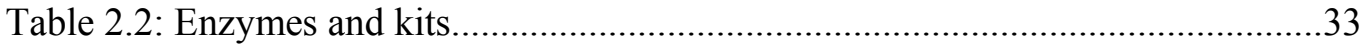

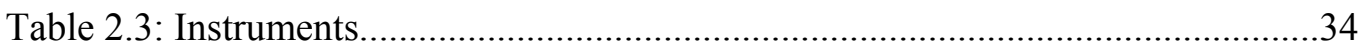

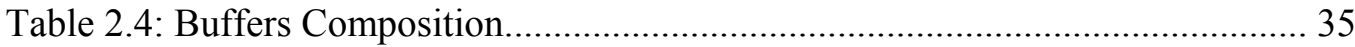

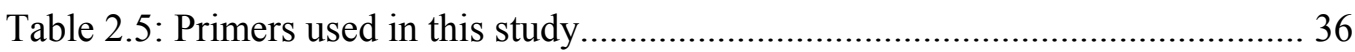

Table 2.6: Restriction digestion reaction mix....................................................... 40

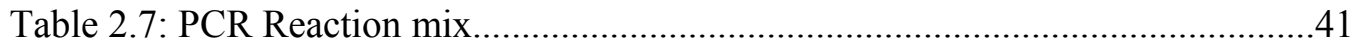

Table 2.8: Temperature cycles for DNA amplification by PCR ................................ 41

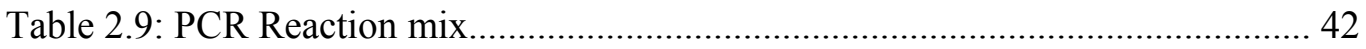

Table 2.10: Temperature cycles for DNA amplification for site-directed

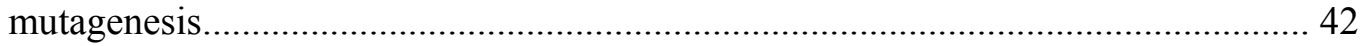

Table 2.11: Extinction co-efficient and molecular weight..................................... 47

Table 7.1: Comparison of the in vitro data on thermostability of Rca among higher plants................................................................ 153

Table 7.2: Summary of rice activase mutants evaluated in this study......................160

Table 8.1: List of species and accession numbers for Rca2 and Rca sequences from plants and green algae used for the phylogenetic analysis.......................................173 


\section{Abbreviations}

\begin{tabular}{|c|c|}
\hline $2 \mathrm{PG}$ & 2-Phosphoglycolate \\
\hline $3 \mathrm{PG}$ & 3-Phosphoglycerate \\
\hline AAA+ & ATPase associated with various cellular activities \\
\hline ADP & Adenosine 5'-diphosphate \\
\hline APS & Ammonium persulphate \\
\hline A. tequilana & Agave tequilana \\
\hline ATP & Adenosine 5'-triphosphate \\
\hline ATP $\gamma \mathrm{S}$ & Adenosine 5'-thiotriphosphate \\
\hline AtRca & Agave tequilana Rubisco activase \\
\hline CA1P & 2-Carboxy-arabinitol 1-phosphate \\
\hline CABP & 2-Carboxy-arabinitol-1,5-bisphosphate \\
\hline CAM & Crassulacean acid metabolism \\
\hline $\mathrm{CCM}$ & Carbon concentrating mechanism \\
\hline $\mathrm{CD}$ & Circular dichroism \\
\hline $\mathrm{CO}_{2}$ & Carbon dioxide \\
\hline$\Delta$ & Deletion \\
\hline DTT & Dithiothreitol \\
\hline E.coli & Escherichia coli \\
\hline ECM & Carbamylated/activated Rubisco \\
\hline ER & RuBP-inhibited Rubisco \\
\hline EDTA & Ethylenediaminetetraacetic acid \\
\hline EM & Electron microscopy \\
\hline HEPES & N-(2-hydroxyethyl)piperacin-N'-2-ethanesulfonic acid \\
\hline $\mathrm{His}_{6} \mathrm{Ub}$ & Hexa-histidine tagged ubiquitin \\
\hline IMAC & Immobilized metal ion affinity chromatography \\
\hline IPTG & Isopropyl $\beta$-D-thiogalactopyranoside \\
\hline LB & Lysogenic broth media \\
\hline $\mathrm{Mg}^{2+}$ & Magnesium ion \\
\hline $\mathrm{MgCl}_{2}$ & Magnesium chloride \\
\hline $\mathrm{NaCl}$ & Sodium chloride \\
\hline
\end{tabular}




\begin{tabular}{|c|c|}
\hline $\mathrm{NAD}+/ \mathrm{NADH}$ & Nicotinamide adenine dinucleotide oxidized / reduced \\
\hline $\mathrm{NADP}+$ / NADPH & $\begin{array}{l}\text { Nicotinamide adenine dinucleotide phosphate oxidized } \\
\text { /reduced }\end{array}$ \\
\hline Native-PAGE & Native polyacrylamide gel electrophoresis \\
\hline Ni-NTA & Nickel-Nitrilotriacetic acid \\
\hline OD & Optical density \\
\hline O. sativa & Oryza sativa \\
\hline OsRca & Oryza sativa Rubisco activase \\
\hline PCR & Polymerase chain reaction \\
\hline $\mathrm{PEP} / \mathrm{C}$ & Phosphoenolpyruvate/Carboxylase \\
\hline PMSF & Phenylmethylsulfonylfluoride \\
\hline Rbc & Rubisco \\
\hline $\mathrm{RbcL}$ & Rubisco large subunit \\
\hline $\mathrm{RbcS}$ & Rubisco small subunit \\
\hline Rca & Rubisco activase \\
\hline RT & Room temperature \\
\hline Rubisco & Ribulose 1,5-bisphosphate carboxylase/oxygenase \\
\hline RuBP & Ribulose 1,5-bisphosphate \\
\hline SDS-PAGE & $\begin{array}{l}\text { Sodiumdodecylsulphate polyacrylamide gel } \\
\text { electrophoresis }\end{array}$ \\
\hline SEC & Size exclusion chromatography \\
\hline TAE & TRIS-acetate-EDTA \\
\hline TEMED & $\mathrm{N}, \mathrm{N}, \mathrm{N}^{\prime}, \mathrm{N}^{\prime}$-tetramethylethylenediamine \\
\hline Tris & Tris-(hydroxymethyl)-aminomethane \\
\hline TV & PGemT Vector \\
\hline $\mathrm{Ub}$ & Ubiquitin \\
\hline Usp2 & Ubiquitin specific-protease 2 \\
\hline WT & Wild-type \\
\hline
\end{tabular}




\begin{abstract}
Ribulose 1,5-bisphosphate carboxylase/oxygenase (Rubisco) forms inhibited complexes with its own substrate RuBP and other sugar phosphates. The AAA+ protein Rubisco activase (Rca) counteracts this issue by removing these inhibitors and thus maintaining Rubisco in its functional state. Inactivation of Rca at moderately high temperatures is associated with the loss of the activation state of Rubisco. Here we present a detailed characterization of the Rubisco activase from rice (Oryza sativa). We then compare its properties to a highly functional and thermostable Rca system from the desert plant, Agave tequilana. An extensive mutational study identified a number of residues that are likely involved in the Rubisco-Rca interaction. Finally we present a modified model for Rubisco activation in plants. Our results indicate that bioprospecting and careful biochemical characterization of Rca homologues will lead to both mechanistic insights and identification of candidate proteins for engineering enhanced thermotolerance in rice.
\end{abstract}





\section{Introduction}


The continuously growing human population, reduction of cultivatable land due to urbanisation, and global climate change are threatening our long-term global food security [1]. Collectively these factors represent major challenges for breeders and farmers to maintain food supply in excess of demand. The primary challenge in agricultural research is to increase crop productivity within the available area to sustain the demand of unsustainably growing population in the future. Past and recent efforts involved the green revolution, traditional breeding, genetic engineering, and so on. However, the productivity gains achieved through them are beginning to lag. An important approach to address this problem is to improve the natural efficiency of crop plant photosynthesis $[2,3]$.

Photosynthesis is the basis of life on earth which provides humanity with food, energy and materials. Ribulose 1, 5-bisphosphate carboxylase/oxygenase (Rubisco) is a key enzyme of photosynthesis, responsible for $\mathrm{CO}_{2}$ fixation in the majority of photosynthetic organisms including crop plants. In spite of its important role in nature, Rubisco is a rather slow and confused catalyst [4, 5]. Its inability to differentiate between $\mathrm{CO}_{2}$ and $\mathrm{O}_{2}$ gives rise to a set of side reactions called photorespiration, which result in reduced photosynthetic efficiency [6]. Apart from this, Rubisco is also prone to inhibition by its own substrate RuBP (ribulose 1,5bisphosphate) and other sugar phosphates [7]. In plants, this problem is alleviated by a molecular motor, the AAA+ ATPase Rubisco activase (Rca) $[8,9]$.

Rca performs remodelling of Rubisco and facilitates the release of sugar phosphate inhibitors in an ATP dependent manner. However, Rca is a thermolabile enzyme and this property is believed to be directly associated with the inhibition of photosynthesis at moderately high temperature $[10,11]$. There is strong evidence that expressing more thermostable forms of Rca can lead to improved photosynthetic performance at supraoptimal temperatures $[12,13]$. Due to such findings and its critical position in regulating the carbon fixation process, Rca is currently considered as target for crop improvement strategies $[14,15]$. However, other opinions suggest that the deactivation of Rubisco at elevated temperature may instead be an important regulatory response to protect the plant during heat stress $[16,17]$.

The molecular mechanism underlying the thermolability of photosynthesis is of particular interest due to the predicted effects of global climate change [18]. Detailed 
biochemical and structural characterization of the system's components will assist in clarifying these issues. A comprehensive mechanistic understanding of an agriculturally relevant Rubisco regulation system in plants like Oryza sativa will develop our ability to predict and modify the behaviour of their carbon fixation machinery. Ultimately, this will provide the new avenue for future crop improvement derived from such work.

\subsection{Photosynthesis}

Photosynthesis is the process of conversion of light energy into chemical energy, resulting in the formation of organic compounds from carbon dioxide and water using energy from the sun. It is one of the fundamental processes on earth, performed by plants, algae and some specialised bacteria [19]. It is not only a source of energy for autotrophs but also for all other non-photosynthetic organisms including ourselves that are unable to produce organic materials from inorganic carbon sources. Thus, almost all living beings directly or indirectly depend on photosynthesis for their survival.

Photosynthesis is a compartmentalized process that takes place in the chloroplast of plants and consists of the light reactions (photosynthetic electron transport) which take place in the thylakoid membranes and the dark reactions (photosynthetic carbon metabolism) in the stroma [20]. During the light reactions, light energy from the sun is captured by chlorophyll and converted into chemical energy in the form of NADPH and ATP, with the liberation of molecular oxygen. The dark reactions, also known as the Calvin-Benson cycle utilize these molecules for the carbon fixation and production of carbohydrates (sugars). 


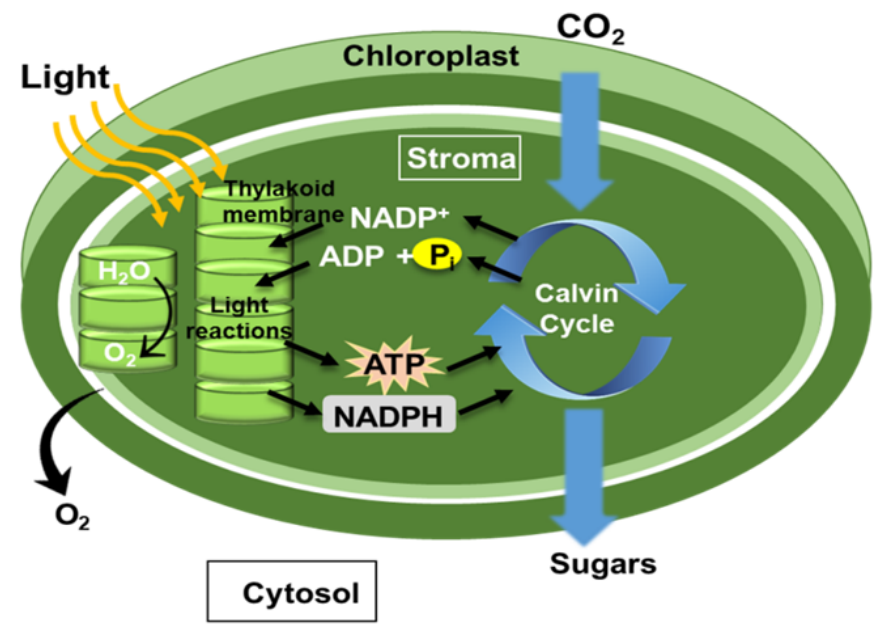

Fig. 1.1: Schematic illustration of the basic process of photosynthesis that takes place in the chloroplast. Modified from [21]

\subsubsection{The light reactions}

These set of reactions take place in the thylakoid membranes of the chloroplast and generate ATP and reducing equivalents from light energy derived from sun. Light in the form of photons is captured by chlorophyll and other pigment molecules (light harvesting complexes) present in the thylakoid membranes. The energy is then utilized to generate a linear electron flow from photosystem (PS) II to I (reaction centres). The electron transport chain begins with oxidation of water molecule yielding energetic electrons used to reduce $\mathrm{NADP}^{+}$to NADPH in the stroma. The electron flow also drives the generation of a chemiosmotic gradient that is utilized to phosphorylate ADP to form ATP (Fig. 1.2A).

The process initiates with the concurrent excitation of electrons from specially-bound chlorophyll molecules in the reaction centres of PSI and PSII. The energy cascaded by the pigments in PSI is used by the special P700 chlorophyll (pigment having absorption maxima at $\lambda \sim 700 \mathrm{~nm}$ ), to excite an electron to a higher energy level $\left(\mathrm{P} 700^{*}\right)$, which enables an adjoining electron acceptor molecule to capture the excited electron. Electrons derived from P700 are eventually passed down to adjacent lower energy carrier molecules and reaches the ultimate electron carrier $\mathrm{NADP}^{+}$, creating an electron transport chain. Two electrons transported in the system combine with $\mathrm{H}^{+}$ ions and $\mathrm{NADP}^{+}$to form NADPH (Fig. 1.2A). The electron deficient $\mathrm{P}^{+} 00^{+}$is 
replenished by electrons originating from PSII. Similar to PSI, light energy excites an electron of the specially-bound P680 chlorophyll molecule so as to be absorbed by the neighbouring electron acceptor molecule. The electrons cascade through a series of carrier molecules in a different electron transport chain to be finally transferred to the $\mathrm{P} 700^{+}$molecule of the PSI and making it functional for the next cycle [19]. The electron transport chain of PSII establishes a proton gradient across the thylakoid membrane which fuels the production of ATP from ADP by chloroplast $\mathrm{F}_{1} \mathrm{~F}_{0}$ ATP synthase [22, 23] (Fig. 1.2A). The electron deficient $\mathrm{P} 680^{+}$, which ultimately contributed the electrons to $\mathrm{P} 700^{+}$, is replaced by the electrons generated by splitting of water into hydrogen ions $\left(\mathrm{H}^{+}\right)$and oxygen gas $\left(\mathrm{O}_{2}\right)$ [24]. These electrons are used to regenerate P680 of PSII. Electron transport between two photosystems through the electron transport chain complex to reduce $\mathrm{NADH}^{+}$is also referred as the " $\mathrm{Z}$ scheme" (Fig. 1.2B). Protons are pumped into the thylakoid lumen to generate the proton gradient powering ATP synthesis. This is driven by cyclic or non-cyclic electron transport across the photosystems $[22,23]$.

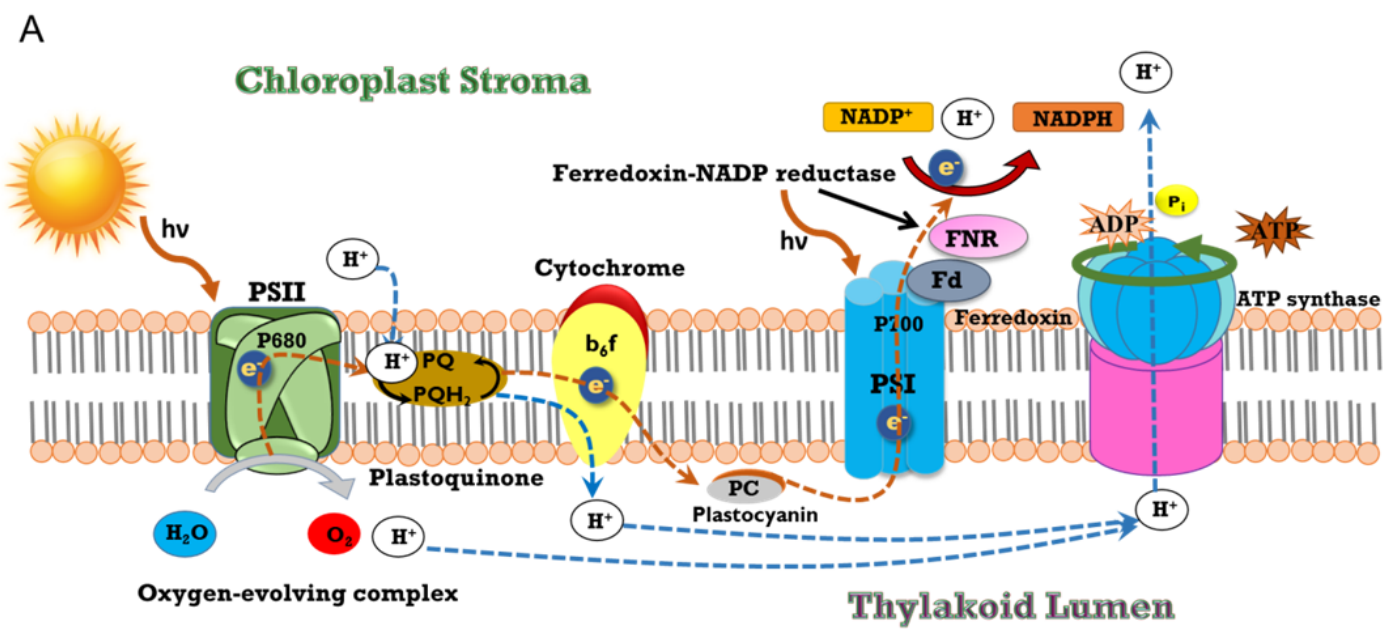




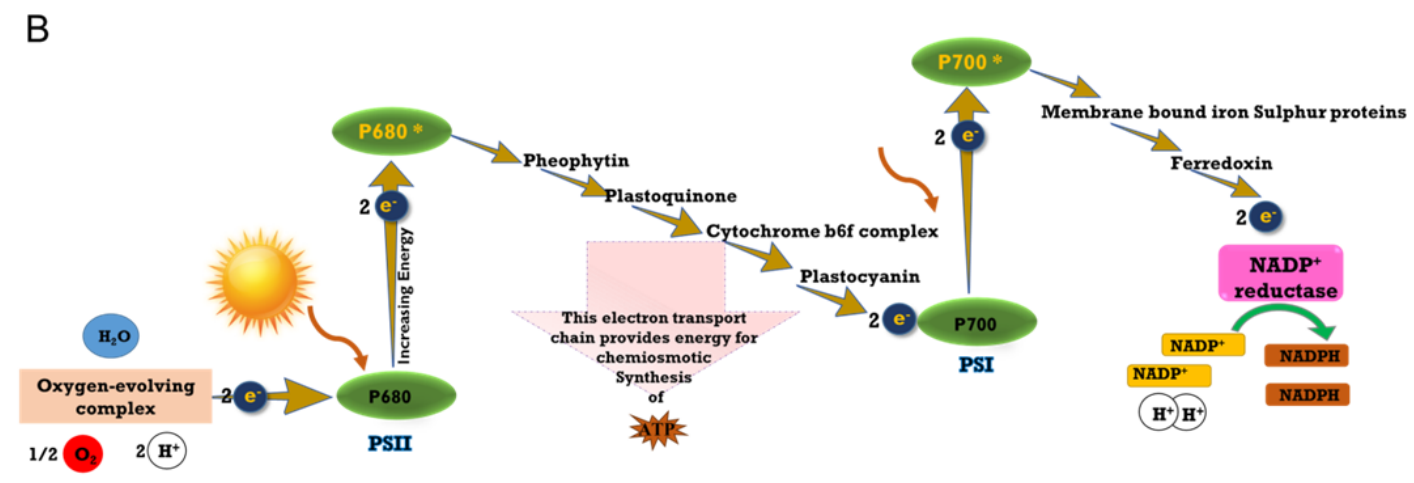

Fig. 1.2: Light dependent reactions of photosynthesis. (A) Schematic representation of the events taking place during the light reactions. The electron transport chain created across the thylakoid membrane from PSII to PSI ultimately reducing NADP ${ }^{+}$to NADPH. (B) Z-scheme of the linear electron flow across two photosystems. Electrons are derived from the oxidation of water molecules at the oxygen evolving complex (OEC) and pass through PSII to plastoquinone (PQ/PQH $)$. Oxidation of $\mathrm{PQH}_{2}$ occurs at the cytochrome b6f complex (b6f) where half of the electrons are linearly transferred to $\mathrm{NADP}^{+} / \mathrm{NADPH}$ coupled via plastocyanin (PC), PSI, ferredoxin (Fd), and ferredoxin-NADP+ oxidoreductase (FNR). The other half of the electrons returns to the $\mathrm{PQH}_{2}$ pool. Protons released at the $\mathrm{OEC}$ and the cyclic reduction and oxidation of $\mathrm{PQ} / \mathrm{PQH}_{2}$ set up a protons gradient across the thylakoid membrane. This chemiosmotic potential is used to produce ATP from ADP at the chloroplast $\mathrm{F}_{1} \mathrm{~F}_{0}$ ATPase. Modified from [19, 23]

\subsubsection{The dark reactions (The Calvin-Benson cycle)}

The Calvin-Benson or Calvin-Benson-Bassham cycle, named so after their discoverers [25], consists of a series of enzymatic oxidation-reduction reactions. Fig. 1.3 represents the schematic overview of the pathway. This pathway encompasses three phases: carbon fixation, reduction, and regeneration of substrate. Carbon fixation is the first step in which RuBP is carboxylated in the presence of atmospheric $\mathrm{CO}_{2}$ to produce two molecules of 3-phosphoglycerate (3PG). This reaction is catalysed by the world's most abundant enzyme, ribulose 1, 5-bisphosphate carboxylase/oxygenase (Rubisco) [26, 27]. The reduction step consists of a series of reactions utilizing the end products from the light reactions (ATP and NADPH) to phosphorylate 3PG to 1, 3-bisphosphoglycerate, which is subsequently reduced and dephosphorylated to glyceraldehydes 3-phosphate (G3P). In the regeneration phase, RuBP is regenerated by using carbon from G3P. This is accomplished by a series of carbon reshuffling reactions. In the final reaction, the precursor ribulose 5- phosphate is regenerated in an ATP-dependent manner to RuBP by phosphoribulokinase. Fivesixths of the G3P is used for the synthesis of sugars, which are then used to make all of the organic molecules needed by the plant. Overall, the Calvin-Benson cycle 
generates one molecule of G3P from three molecules of $\mathrm{CO}_{2}$ at the cost of nine molecules of ATP and six molecules of NADPH [28]. The net reaction of CalvinBenson cycle is therefore:

$$
\begin{array}{r}
3 \mathrm{CO}_{2}+5 \mathrm{H}_{2} \mathrm{O}+6 \mathrm{NADPH}+9 \mathrm{ATP}+3 \mathrm{RuBP} \longrightarrow \mathrm{G} 3 \mathrm{P}+3 \mathrm{H}^{+}+6 \mathrm{NADP}^{+}+9 \mathrm{ADP} \\
+8 \mathrm{P}_{\mathrm{i}}+3 \mathrm{RuBP}
\end{array}
$$

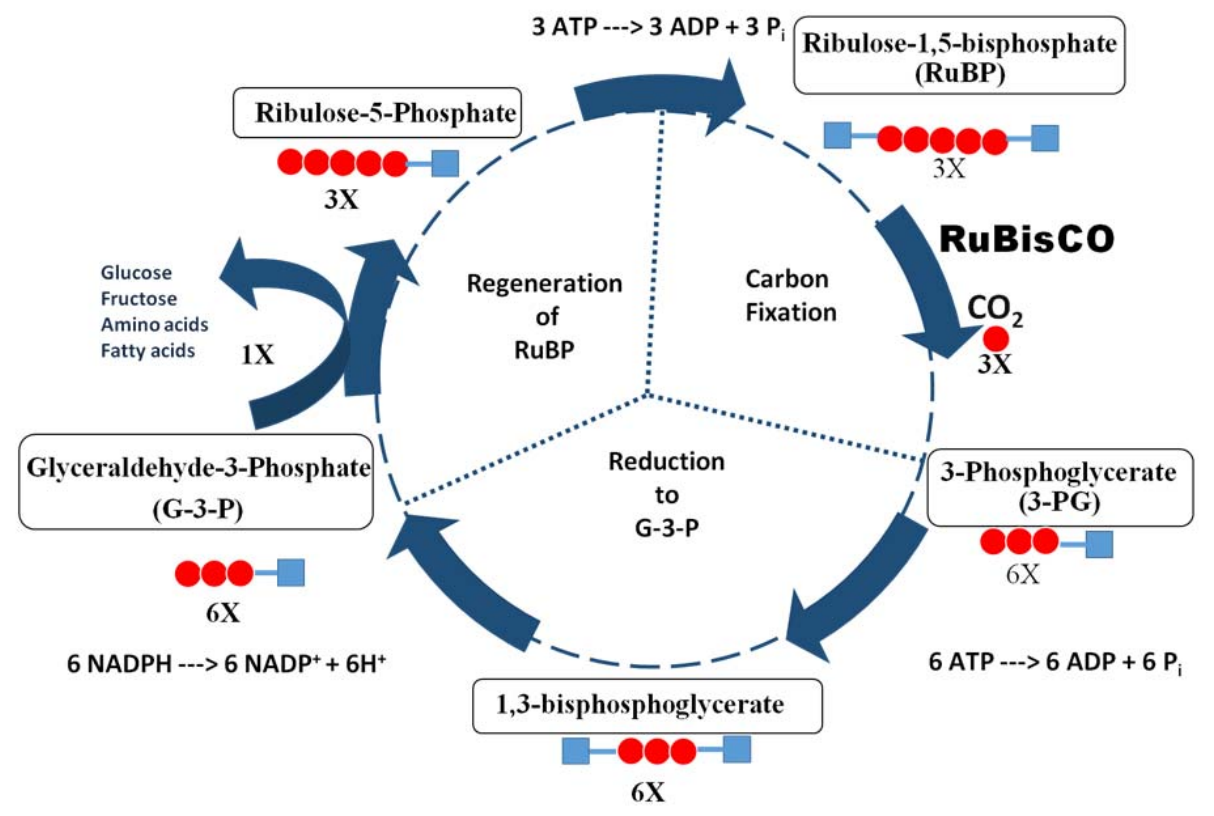

Fig. 1.3: The Calvin-Benson cycle. RuBP (5-carbon molecule) with the addition of $\mathrm{CO}_{2}$ forms an unstable 6-carbon molecule (Carbon fixation phase). This 6-carbon molecule immediately breaks down into two 3-carbon molecules (3-PG) which is phosphorylated to form 1,3-bisphosphoglycerate molecules and subsequently reduced to form G-3-P by the consumption of ATP and NADPH (Reduction phase). Some of the G-3-P formed is shunted out of Calvin-Benson cycle to produce glucose (Carbohydrate formation) and other, more complex organic molecules. The remaining glyceraldehyde-3-phosphate is used to regenerate the 5-carbon $\mathrm{CO}_{2}$ acceptor molecule by a series of reactions (Regeneration phase).

\subsubsection{Photorespiration}

Rubisco also catalyzes the oxygenation of RuBP under low $\mathrm{CO}_{2}$ conditions. This futile side reaction leads to formation of one molecule of 2-phosphoglycolate (2PG) and one molecule of 3PG [29]. For plants, 2PG is considered a harmful compound, which needs to be converted into 3PG via an energy consuming photorespiratory pathway [30]. It is salvaged by a series of reactions taking place in the chloroplast, cytosol, mitochondria and peroxisome (Fig. 1.4) where it is converted into glycerate and recycled back to the chloroplast to be utilized in Calvin-Benson cycle [6]. 
The cycle begins in the chloroplast stroma where resulting 2PG from the oxygenation reaction is dephosphorylated by 2-phosphoglycolate phosphatase (PGP) into glycolate. This glycolate is transported from the chloroplast to the peroxisome where a peroxisomal enzyme glycolate oxidase (GOX) oxidized it into glyoxylate. The produced glyoxylate is transaminated into glycine by serine-glyoxylate transaminase (SGT) and glutamate-glyoxylate transaminase (GGT). The glycine then enters the mitochondria where two molecules of glycine are converted into serine by the action of glycine decarboxylase (GDC) and serine hydroxymethyltransferase (SHMT). Additionally, one molecule of ammonia and $\mathrm{CO}_{2}$ is released. The serine formed, is sent back to the peroxisome and produces hydroxypyruvate by the action of SGT which is subsequently reduced to glycerate by hydroxypyruvate reductase (HPR). Glycerate is exported from peroxisome to the chloroplast, where it is finally converted into 3PG by the plastidial glycerate 3-kinase (GLYK), thus completing the cycle.

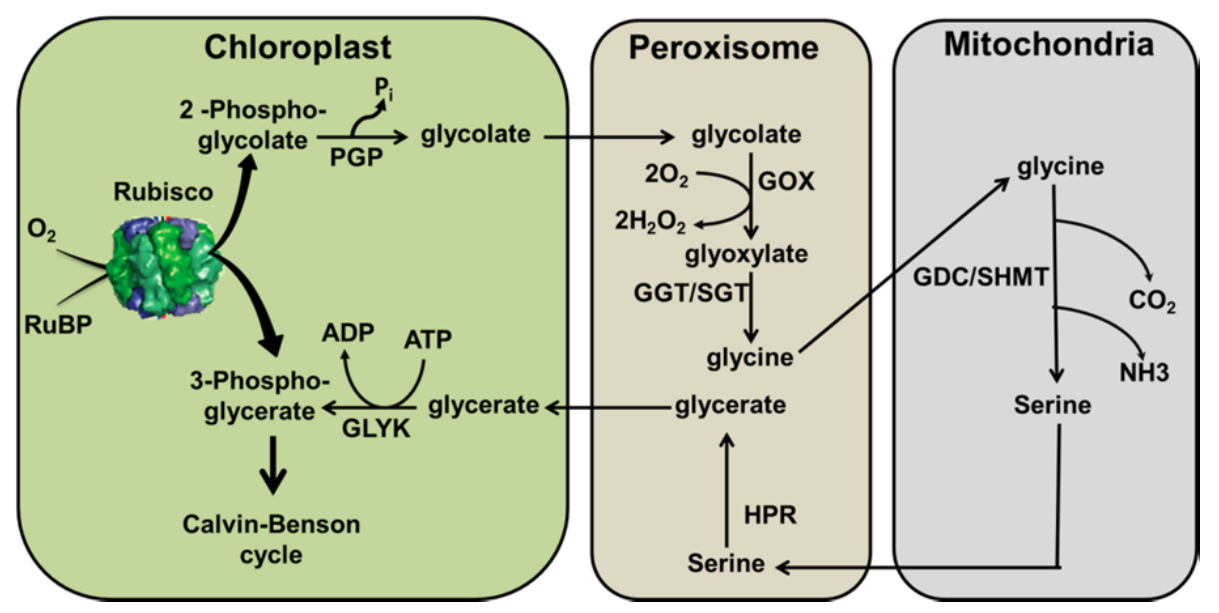

Fig. 1.4: Schematic overview of the photorespiratory pathway. Rubisco catalyzes the oxygenation of RuBP, a major side reaction leading to formation of 2-phosphoglycolate. In plants, this molecule has to be salvaged back to 3-phosphoglycerate by the photorespiratory cycle. The pathway involves a series of reactions taking place in chloroplast, peroxisome and mitochondria as shown in the figure.

The oxygenation reaction costs one ATP molecule and results in loss of one $\mathrm{CO}_{2}$ molecule, reducing the net efficiency of photosynthesis. It has been reported that significant improvement in the photosynthetic efficiency could be achieved if photorespiration is repressed by augmenting carbon dioxide in the proximity of Rubisco using free air $\mathrm{CO}_{2}$ enrichment (FACE) studies [31-33]. Plants grown under a high $\mathrm{CO}_{2}$ fertilized environment in such studies show repressed photorespiration and 
produce more biomass. Also, plants carrying a metabolic bypass for photorespiration demonstrate improved photosynthesis and increased biomass [34, 35]. This seems to provide a promising approach for improving the photosynthetic productivity in many C3 plants that confront a significant drain in metabolic energy due to high rates of photorespiration. However, the growth rate enhancement achieved by plants with repressed oxygenation reactions was smaller than predicted by theoretical models [33], when such plants were grown under a high $\mathrm{CO}_{2}$ environment. Some researchers suggest that reducing photorespiration will have a negative impact on plant physiology. For instance, several studies have reported the importance of photorespiration in processes such as plant defence and nitrate assimilation, hinting at beneficial aspects of photorespiration $[36,37]$.

\subsubsection{Carbon concentrating mechanisms}

The significant loss of metabolic energy caused by operation of the photorespiratory pathway has resulted in a strong evolutionary selection pressure to minimize the oxygenation reactions. Therefore, in spite of contrasting reports on the importance and benefits of photorespiration, many photosynthetic organisms including most aquatic microalgae have evolved efficient carbon concentrating mechanisms (CCM). The multiple independent CCM's are employed by different organisms where Rubisco resides in an environment experiencing an elevated local $\mathrm{CO}_{2}$ concentration.

Cyanobacteria and many proteobacteria possess a specialised micro-compartment enclosed by a proteinaceous, icosahedral shell known as carboxysomes [38]. The shell self-assembles and contains Rubisco and carbonic anhydrase [39]. Inorganic carbon in the form of bicarbonate is pumped into the cells by active transport. The co-localised carbonic anhydrase converts this bicarbonate into gaseous $\mathrm{CO}_{2}$ near the site of Rubisco, effectively increasing local $\mathrm{CO}_{2}$ levels and thus minimises the competing oxygenation reaction.

In most algae, such as Chlamydomonas reinhardtii, Rubisco is placed in a distinct stromal microcompartment known as the pyrenoid [40]. In green algae, the pyrenoid is surrounded by starch-like oligosaccharides unlike the carboxysome [41]. Due to difficulties in purification and stabilization of the pyrenoid, the mechanistic details of the CCM in algae are still elusive. 
Some terrestrial plants have evolved pathways to concentrate $\mathrm{CO}_{2}$ at the Rubisco active site in syndromes known as C4 and CAM photosynthesis [42]. C4 plants such as maize possess a characteristic leaf anatomy called Kranz anatomy. Initial $\mathrm{CO}_{2}$ fixation by PEP carboxylase takes place in specialized cells called mesophyll cells followed by shuttling of fixed carbon in the form of malate or aspartate to bundle sheath cells where the Rubisco is located. Thus Rubisco is isolated from atmospheric oxygen and remains saturated with $\mathrm{CO}_{2}$ released by decarboxylation of these organic acids. Given the advantages of $\mathrm{C} 4$ cycles, initiatives have been taken to incorporate C4 features into $\mathrm{C} 3$ plants like rice $[43,44]$.

CAM plants are adapted to survive in hot and arid conditions. The carbon fixation in such plants takes place during night when ambient temperature is low and the stomata are open allowing $\mathrm{CO}_{2}$ to enter. They remain closed during day to prevent loss of water. The $\mathrm{CO}_{2}$ is fixed into oxaloacetic acid by the action of PEP-carboxylase at night. Subsequently, this oxaloacetate is converted into malate and stored in vacuoles. During day, when temperature is high and stomata are closed to prevent water loss, this malate is shuttled into the chloroplasts and decarboxylated into pyruvate, releasing $\mathrm{CO}_{2}$ which then enters the Calvin-Benson cycle for fixation [45]. CAM photosynthesis reflects a temporal separation of $\mathrm{CO}_{2}$ uptake and fixation, while $\mathrm{C} 4$ photosynthesis represents spatial separation of the two processes.

\subsection{Ribulose 1, 5- bisphosphate carboxylase/oxygenase (Rubisco)}

Rubisco (EC.4.1.1.39) is believed to be the most abundant protein on earth [26] and is the key enzyme of the dark reactions. It often makes up $50 \%$ of the total soluble protein found in plant leaf tissue and accounts for the majority of the protein in some phototrophic microbes $[5,29]$. It catalyses the initial steps of photosynthetic carbon fixation by converting $\mathrm{RuBP}$ and $\mathrm{CO}_{2}$ to two molecules of 3PG. Rubisco has slow catalytic properties and due to its inability to differentiate between $\mathrm{CO}_{2}$ and $\mathrm{O}_{2}$, it also catalyses the oxygenation of RuBP which leads to formation of toxic $2 \mathrm{PG}[4,5]$. This compound has to be converted back to $3 \mathrm{PG}$ at the cost of energy and $\mathrm{CO}_{2}$, which reduces the efficiency of photosynthesis [6]. Apart from this, Rubisco is prone to inhibition by its own substrate and other inhibitory sugar phosphates, some of them generated by its own active site, leading to a phenomenon called fallover [7, 46]. All these drawbacks might have compelled its abundant expression in nature. The 
manipulation of kinetic properties of Rubisco has been the objective for many years and widely attempted for improving photosynthetic yield in higher plants [47, 48]. Rubisco from different species shows different kinetics and substrate specificity. For instance, Rubisco from red algae like Griffithsia monilis show at least three fold higher $\mathrm{CO}_{2} / \mathrm{O}_{2}$ specificity $\left(\mathrm{S}_{\mathrm{C} / \mathrm{O}}\right)$ compared to any other plant Rubisco [49-51].

\subsubsection{The structure and classification of Rubisco}

The Rubisco enzyme found in higher plants consists of large subunits (RbcL) 52 $\mathrm{kDa}$ and small subunits $(\mathrm{RbcS}) \sim 15 \mathrm{kDa}$ in a hexadecameric stoichiometry, $\mathrm{L}_{8} \mathrm{~S}_{8}$ [52]. The fundamental structural unit of all Rubiscos is a dimer of large subunits $\left(\mathrm{RbcL}_{2}\right)$. Rubisco is classified into four major forms namely form I-IV (Fig. 1.5) of which only the first three catalyze RuBP-dependent carbon fixation reactions (carboxylation/oxygenation) [53, 54]. Form IV enzymes termed as Rubisco-like proteins (RLP) and do not catalyse the $\mathrm{CO}_{2}$ fixation reaction [55].
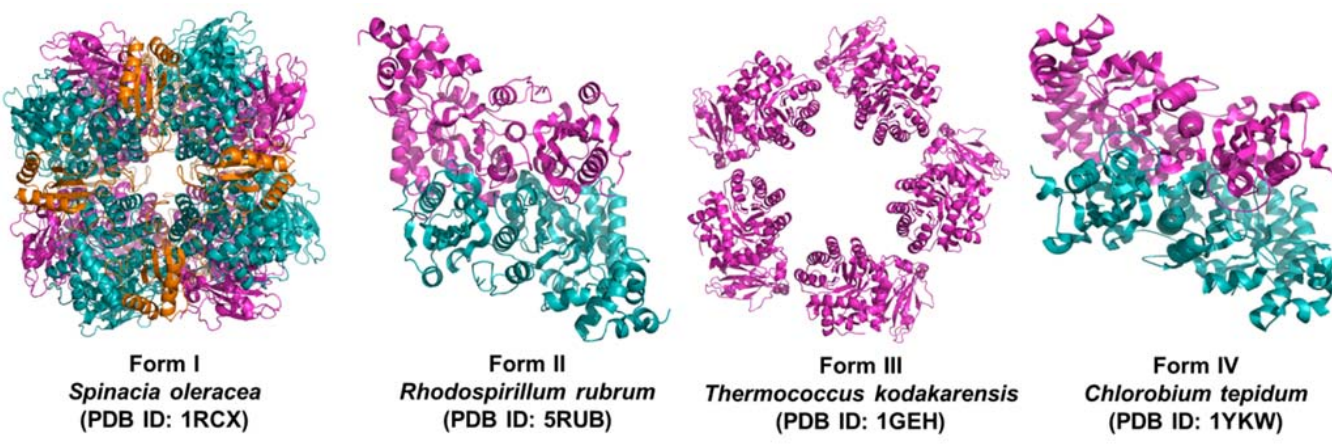

Fig. 1.5: Representatives of the four forms of Rubisco. All forms are made up of the functional units of antiparallel dimers of large subunits. Form I forms tetramers of dimers and is the only form that comprises of the small subunits. Form II is made up of dimers or oligomers $(n=2-5)$, the dimeric form is shown here. Form III Rubisco forms tetramers or pentamers of dimers, shown here is the pentameric form. Form IV enzymes are comprised of a single antiparallel dimer of large subunits. PDB accession numbers are indicated in brackets under the species name $[53,56]$.

Out of the four types, only form I has a small subunit and exists as a hexadecameric complex $\left(\mathrm{L}_{8} \mathrm{~S}_{8}\right)$ of four antiparallel $\mathrm{RbcL}_{2}$ which is crowned by four RbcS subunits at the top and bottom [53]. This is the most common form of Rubisco and is further classified into four sub groups: Form IA-D. Form IA and Form IB are commonly referred to as green-type Rubisco [53] found in plants, green algae, cyanobacteria and proteobacteria. In plants and green algae the $\mathrm{RbcL}$ is encoded by chloroplast, whereas 
$\mathrm{RbcS}$ is encoded by the nuclear genome [57]. Form IC and form ID are referred as Red-type Rubisco [53] in which RbcL and RbcS are encoded in the same operon [58] and is found in some proteobacteria, red algae and other non-green algae.

The overall fold of the RbcL monomer is well-conserved in all forms of Rubisco. It is made up of a $\mathrm{N}$-terminal $\alpha+\beta$ domain and a larger $\mathrm{C}$-terminal $\alpha / \beta$ TIM barrel domain $\left(\beta_{8} \alpha_{8}\right)$ [59]. The active site is at the intradimer $\left(\operatorname{RbcL}_{2}\right)$ interface between the Ndomain of one subunit and the $\mathrm{C}$-domain of the adjacent subunit. Consequently, the functional unit of Rubisco is an $\mathrm{L}_{2}$ dimer with two active sites per dimer [4].

Although the form I RbcL amino acid sequences are highly conserved, the RbcS sequences are more diverse with only $30-40 \%$ homology between different species [59]. Even though they are not strictly required for enzymatic function, the small subunits are important for maximal catalytic rates and substrate specificity [60-62]. The green and red type Rubisco enzymes differ in several structural features, the most notable being an extension in the C-terminal domains of the small subunits in the redtype enzymes. The $\mathrm{C}$-terminal extensions form a small barrel that protrudes into the solvent channel of the holoenzyme [63]. It is possible that the structural variance between the two subclasses could justify the higher substrate specificity for $\mathrm{CO}_{2}$ over $\mathrm{O}_{2}$ of some red-type Rubiscos as compared to green-type enzymes.

Form II Rubisco found in proteobacteria and dinoflagellates is active as $\mathrm{RbcL}_{2}$. In many chemoautotrophs Form II often co-exists with Form I Rubisco with different numbers of RbcL dimeric pairs, $\left(\mathrm{L}_{2}\right) \mathrm{n}$. This form is reported to have a poor $\mathrm{CO}_{2} / \mathrm{O}_{2}$ specificity [52]. Although the residues involved in catalysis are conserved, the sequence homology between From I and Form II large subunits is only 30 \% [52, 64].

Form III Rubisco exists in some thermophilic archaea, which lack the Calvin-Benson cycle but catalyzes the carboxylation reaction of RuBP, which is generated via an alternate pathway [65]. They exist as dimeric structure, however pentamers of dimers $\left(\mathrm{RbcL}_{2-5}\right)$ were also found as active form [66-70].

Form IV Rubisco are known as Rubisco-like proteins (RLP) which forms antiparallel dimers of large subunits similar to Form II however they are not genuine Rubiscos $[56,71]$. Form IV Rubiscos demonstrate dissimilarity in critical residues at active sites and do not catalyse $\mathrm{CO}_{2}$ fixation $[55,72]$. The RLPs from Bacillus subtilis and 
Geobacillus kaustophilus are reported to be involved in a methionine salvage pathway by performing an enolase-type function similar to Rubisco on a different substrate $[71,73]$. The RLP from green sulphur bacteria Chlorobium tepidum is involved in thiosulphate oxidation [72].

\subsubsection{Reaction mechanism: carbamylation and catalysis}

The Rubisco apo form (E) requires two cofactor, $\mathrm{CO}_{2}$ and $\mathrm{Mg}^{2+}$, to bind at the active site to perform its catalytic function [74]. The active site for carboxylation is present at the interface between $\mathrm{N}$-terminal domain of one subunit and the $\mathrm{C}$-terminal domain of the adjacent subunit of the antiparallel RbcL2 [75]. Activation takes place by the binding of a non-substrate $\mathrm{CO}_{2}$ molecule with the $\varepsilon$-amino group of lysine 201 (spinach numbering) located at the catalytic sites to form a carbamate (EC). The carbamate is stabilized by the addition of $\mathrm{Mg}^{2+}$ to form the active complex (ECM) $[74,76]$. These spontaneously occurring, reversible reactions are only possible in the 'open state' of the enzyme, which allows solvent access to the binding pockets [47]. The addition of $\mathrm{Mg}^{2+}$ at the Rubisco active site is important for the positioning of the RuBP for the electrophilic attack of the substrate $\mathrm{CO}_{2}$ molecule [5]. The substrate $\mathrm{RuBP}$ can then proceed to bind at the active site, triggering the closure of $\alpha / \beta$ TIMbarrel loop 6 over the active site. The extended conformation of loop 6 is then fixed in position by the flexible $\mathrm{C}$-terminal tail of $\mathrm{RbcL}$, excluding the active site from solvent $[77,78]$. Rubisco then proceeds with the carboxylation of RuBP. The active site opens and closes during every catalytic cycle, mostly involving movement of loop 6 [79].

During catalysis, in the 'closed state', loop 6 forms a latch- like structure on top of the substrate and reaction intermediates that are deeply buried in the active site of the complex. Carboxylation is initiated when the carbamylated K201 abstracts a proton from RuBP to form the enediol isomer of RuBP (enolisation) at the active site [29, 76]. This enediol form makes a nucleophilic attack on the substrate $\mathrm{CO}_{2}$ molecule to form a ketonic acid intermediate. This unstable 6-carbon product intermediate of carboxylation is subsequently hydrated and cleaved to produce the two molecules of 3PG [80]. The oxygenation reaction takes place when $\mathrm{O}_{2}$ molecules compete at the active site instead of $\mathrm{CO}_{2}$ molecules. The mechanism is similar to $\mathrm{CO}_{2}$ fixation where the enediol isomer makes a nucleophilic attack on the $\mathrm{O}_{2}$ molecule resulting in an intermediate, which is hydrolysed to form one $3 \mathrm{PG}$ and one 2PG molecule [80]. The 
energy released from the bond cleavage between the two phosphate substituents is likely required to permit the active site to open, allowing release of the products at the end of catalysis. [81].

A

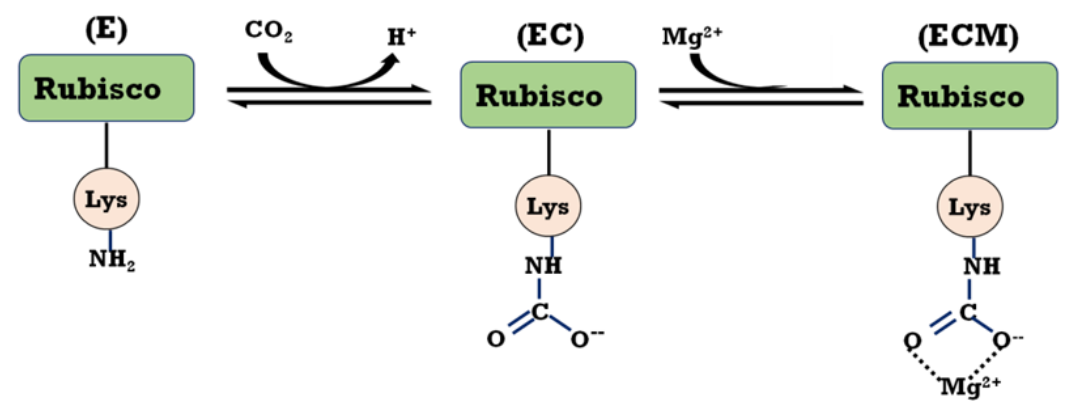

B
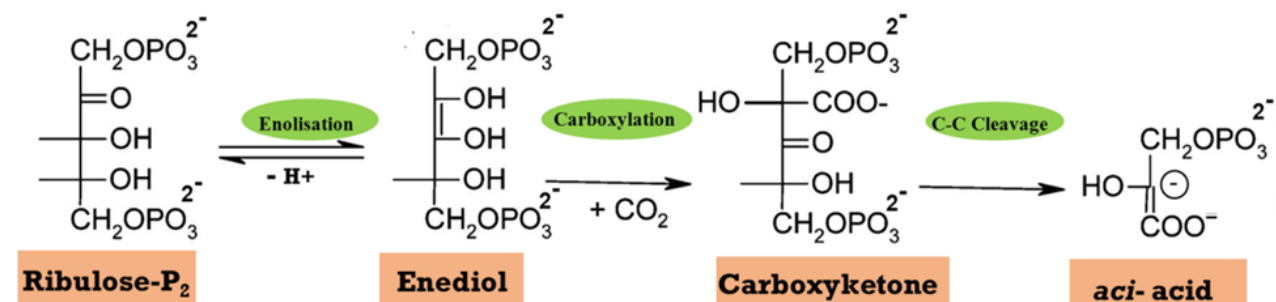

Enediol

Carboxyketone

aci- acid
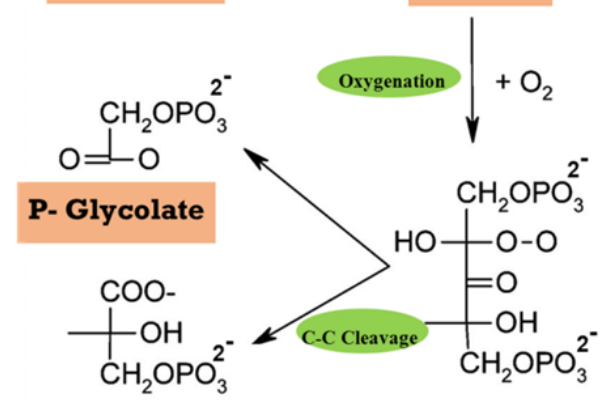

Peroxyketone

P- Glycerate

Fig. 1.6: The carboxylation and oxygenation reaction catalyzed by Rubisco. (A) Carbamylation of Rubisco by $\mathrm{CO}_{2}$ and $\mathrm{Mg}^{2+}$. Rubisco is activated after carbamylation of the $\varepsilon$-amino group of Lys201 by a non-substrate $\mathrm{CO}_{2}$ and binding of $\mathrm{Mg}^{2+}$ to the active site. $\mathrm{Mg}^{2+}$ orients $\mathrm{RuBP}$ and substrate $\mathrm{CO}_{2}$ to be fixed at the active site. (B) Carboxylase activity of Rubisco. RuBP forms an enediolate intermediate. $\mathrm{CO}_{2}$ is polarized by the amino group of lysine 334 and undergoes nucleophilic attack by the enediolate, producing a branched six-carbon intermediate. C-3 of this intermediate is hydroxylated by a water molecule forming a $\beta$-keto acid intermediate. One molecule of 3PG is formed as a result of aldol cleavage. Oxygenase activity of Rubisco. Addition of $\mathrm{O}_{2}$ to RuBP results in an unstable intermediate that splits into two molecules, 2PG and 3PG. Modified from [19, 82] 


\subsubsection{Rubisco inhibition and regulation}

Rubisco is known to form inhibited complexes with its own substrate RuBP and a number of other sugar phosphates. Binding of RuBP to the decarbamylated form of Rubisco results in an inhibited complex known as ER which is catalytically inactive [83]. Additionally, several misfire products generated during catalysis bind to noncarbamylated Rubisco and prevent catalysis [7]. In many plants other naturally occurring sugar phosphates such as the nocturnal inhibitor 2-carboxy-arabinitol-1phosphate (CA1P) bind to the carbamylated form to make stable inactive complexes (Fig. 1.7) [84, 85].

The affinity for RuBP is low in the carbamylated state in order to facilitate cleavage and release at the active site, while under decarbamylated state the affinity is high and therefore Rubisco becomes inactive in decarbamylated state [86, 87]. Thus, different inhibitors bind to Rubisco in the carbamylated and non-carbamylated state with different affinities. RuBP and its misfire products like D-xylulose 1,5-bisphosphate (XuBP), glycero-2,3-pentodiulose-1,5-bisphosphate (PDBP) favours binding to the non-carbamylated enzyme (E) to form the non-carbamylated inhibited complex (EI). Other inhibitors such as CA1P and the non- physiological transition state analogue 2carboxy-D-arabinitol 1,5-bisphosphate (CABP) binds to carbamylated active Rubisco (ECM) and form a carbamylated inhibited complex (ECMI) (Fig. 1.7) [88, 89]. Rubiscos with ligands occupying the substrate binding sites in carbamylated and uncarbamylated state are structurally similar, indicating that cofactors do not play a critical role in substrate binding to the active sites $[90,91]$. Thus, irrespective of whether RuBP or slowly cleavable misprotonation products like XuBP bind in decarbamylated state or CA1P, CABP binds to either form, there is no favourable route for opening and release of product exists. The satisfactory path only exists for ligand RuBP in carbamylated and Mg-bound state for reopening of closed complexes [81]. For instance, a half-life of 530 days was determined for the release of CABP from carbamylated Rubisco in spinach, much higher than other inhibitors, making the binding almost irreversible [88, 92]. 


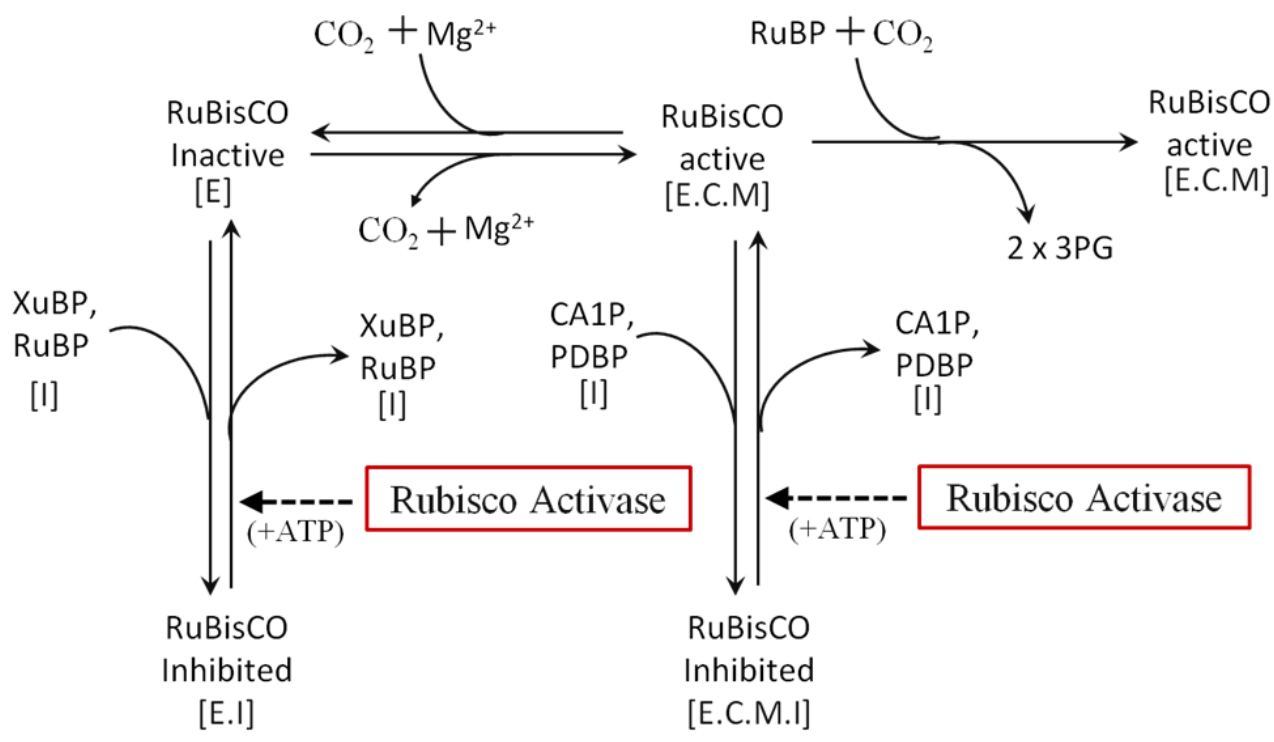

Fig. 1.7: Regulation of Rubisco by Rubisco activase. (A) Decarbamylated Rubisco (E) binds to $\mathrm{CO}_{2}$ and $\mathrm{Mg}^{2+}$ to become the active holoenzyme (E.C.M) which actively fixes $\mathrm{CO}_{2}$ by carboxylation of its substrate RuBP. E form can also bind substrate RuBP or inhibitors to form a dead inhibited complex (E.I). E.C.M can also become inhibited by its nocturnal inhibitor 2-carboxy-arabinitol-1- phosphate (CA1P) or other substrate analogue, D-glycero-2,3-pentodiulose-1,5-bisphosphate (PDBP), 3ketoarabinitol-1,5bisphosphate (KABP) to form inhibited complex (E.C.M.I). The helper enzyme Rubisco activase remodels the Rubisco in both situations by removing the inhibitory sugar-phosphates. Modified from [7].

The inhibition of Rubisco activity due to binding of sugar phosphates in the plant is part of the physiological regulation of the enzyme in vivo; however activity of activated Rubisco also declines in a non-linear manner under in vitro assay conditions. This phenomenon is known as 'fallover' [93-96]. It is the outcome of the accumulation of misfire products during catalysis of RuBP (oxygenation or carboxylation) which block the active sites and inhibit Rubisco activity. The presence of non-cleavable inhibitors also blocks Rubisco active sites. Although they are present in much smaller quantities compared to RuBP, they can form slow tightly bound inhibited complexes [96-98]. Therefore, when active photosynthesis takes place, these inactive Rubisco complexes would accumulate and obstruct $\mathrm{CO}_{2}$ fixation. The fallover is less severe at high temperature due to loose binding of inhibitors in spite of a higher rate of formation of misfire products [99]. This could be the outcome of enhanced flexibility of the Rubisco active site at higher temperature leading to an increased rate of release of inhibitors from the active site. 
The tendency of inhibition by such mechanisms differs between Rubisco from phylogenetically diverse sources $[82,100,101]$. Generally there exists a compromise between substrate specificity $\left(\mathrm{S}_{\mathrm{c} / \mathrm{o}}\right)$ and catalytic rate of the Rubisco in different species $[102,103]$. According to the prevailing model, Rubisco enzyme exhibiting higher substrate specificity favours tight binding of transition state intermediates of carboxylation and consequently the cleavage of tightly bound reaction intermediates becomes slower. Tight binding favours carboxylation over oxygenation but the slower rate unfortunately also favours aggravated misfire product formation [103]. Also, highly selective enzymes form slow-tight binding complexes with inhibitors, indicating that the active sites of these enzymes are more rigid. For instance, plant Rubiscos possessing high specificity, forms inhibited complexes with RuBP and display fallover. Rubisco of red algae with high specificity do not exhibit fallover inhibition, but when inhibited complexes are formed, they are extremely stable [82, 100]. Recently, a red algal Rubisco from Cyanidioschyzon merolae was reported to form a stable inhibited complex only at high temperature $\left(45^{\circ} \mathrm{C}\right)[104]$ that did not reactivate spontaneously at $25{ }^{\circ} \mathrm{C}$. Rubisco from green algae with intermediate specificity forms the inhibited complex with RuBP but does not show fallover when assayed [101]. Whereas, less specific Rubisco from cyanobacteria neither form inhibited ER complexes nor display fallover $[82,101,105,106]$. Thus, highly specific Rubiscos exhibiting slower catalytic rates are particularly prone to form highly stable dead-end inhibited complexes.

In order to overcome this problem of inhibition, during the course of evolution, nature has recruited a molecular chiropractor of Rubisco called Rubisco activase (Rca) [9, 107]. Rca utilizes energy from ATP hydrolysis to perform its function; subsequently other phosphatases assist in degradation of non-RuBP inhibitors [85, 108, 109]. Fig. 1.7 shows the schematic representative mechanism involved in Rubisco regulation by Rca. Since Rca is responsible for maintaining the activation state of Rubisco, various evidence has been put forward after its discovery that proves that Rca is critical to maintain Rubisco functionality in vivo $[7,8]$. Rca has been found to exist in all the plant species surveyed and belongs to the AAA+ family of proteins, which are known to execute chaperone- like functions in other homologous and heterologous systems [29]. 


\subsection{Rubisco activase (Rca)}

Rubisco activase was discovered as an act of serendipity, derived from large scale screens of arabidopsis mutants that required high $\mathrm{CO}_{2}$ for growth. It was observed that the strain was unable to grow at ambient $\mathrm{CO}_{2}$ level and accumulated RuBP due to lack of activation of the Rubisco, but survived at high $\mathrm{CO}_{2}$ levels $[110,111]$. This strain was therefore named as rca for Rubisco activation. Later, it was found that two polypeptide bands were missing on a two dimensional polyacrylamide gel electrophoresis in the $r c a$ mutant during analysis of soluble polypeptides from the arabidopsis mutants and wild-type strains. Biochemical and genetic analysis confirmed that the two missing polypeptides were responsible for the rca mutant phenotype and this protein was later named as Rubisco activase [9].

Identifying the presence of Rca in soluble protein extracts of various $\mathrm{C} 3$ and $\mathrm{C} 4$ plants species confirmed that Rca-dependent regulation of Rubisco was pervasive in higher plants [112]. In the same year, it was also discovered that the Rca is an ATPdependent remodeler of Rubisco [113], which promotes the release of bound sugar phosphates from the active site of both carbamylated and decarbamylated forms of Rubisco [107, 109]. Addition of Rca to uncarbamylated Rubisco showed no activity loss or to carbamylated Rubisco showed no fallover. Thus, it applies to Rubisco inhibited by CA1P or other inhibitors that are formed during catalysis apart from RuBP alone. However, Rubisco activase is not directly involved in carbamylation or binding of magnesium ion at the active site [107].

Twenty-five years after the discovery of green-type Rca in plants, in 2011 a red-type Rubisco activase $(\mathrm{CbbX})$ from $\alpha$-proteobacteria was reported [114]. Last year, a CbbQO complex (purple-type) was found to be functional as Rubisco activase in chemoautotrophic bacteria [115]. Recently, a hetero-oligomeric red-type Rubisco activase was described in a thermophilic red alga $C$. merolae that operates as an hetero-oligomeric complex of a plastid and nuclear-encoded CbbX isoform (CmNP) [104]. Although all these Rubisco activases employ distinct mechanisms, the energy required to remodel inhibited Rubisco complexes is generated by an AA+ protein module. Therefore, in order to generate a foundation for functional understanding of Rubisco activase, it is necessary to appreciate the basic structural and functional aspects of $\mathrm{AAA}+$ proteins in different systems. 


\subsubsection{Rubisco activases are members of the AAA+ family of proteins}

A sequence comparison with related ATPases revealed that Rca belongs to the family of AAA+ (ATPases associated with diverse cellular activities) ring-shaped p-loop ATPase motor proteins [116]. AAA+ proteins as the name suggests, have been described to perform an array of functions at the cellular level and are ubiquitously found across kingdoms of life. The overarching theme in the function of AAA+ proteins involves conformational remodelling of their target substrate (a macromolecule such as protein or polynucleotides) by utilizing energy from ATP hydrolysis $[117,118]$. They have an active role in various cellular processes such as protein folding and degradation, DNA replication, membrane fusion, vesicle trafficking, transcriptional activation, motor activity etc. [117-119]. Class I AAA+ proteins (e.g. ClpA, ClpB, ClpC and HSP104) contain two highly conserved AAA domains, referred to as AAA-1 (or D1) and AAA-2 (or D2), separated by a variable length linker sequence. Class II proteins (e.g. ClpX, HslU), including all known Rubisco activases, contain only a single AAA domain $[117,120]$.

$\mathrm{AAA}+$ proteins consist of a distinct set of conserved sequences and are characterized by nucleotide binding domain (NBD) consisting of a N-terminal $\alpha / \beta$ Rossman fold and a less conserved C-terminal 4-helix bundle (hb) subdomain [117]. An $\alpha / \beta$ Rossman fold forms the core of the N-terminal subdomain with a $\beta$-sheet of parallel strands, arranged in a $\beta 5-\beta 1-\beta 4-\beta 3-\beta 2$ fashion at its core (Fig. 1.8B). This large AAA+ subdomain comprises the signature Walker A, B and sensor I motifs [121] essential for nucleotide binding and hydrolysis. The nucleotide binding domain also contains conserved arginine residues called arginine fingers for intersubunit communication which affects ATP hydrolysis and subunit oligomerization [122]. Another typical feature of the AAA+ proteins are the pore loops facing the central cavity that is lined by residues from the individual subunits [117]. The underlying mechanism of action often involves protein threading, where the substrate protein is translocated through a central pore formed by a ring shaped oligomer of AAA+ protein subunits [123-125]. In the $\alpha$-helical subdomain, a conserved sequence motif called sensor II, characterized by a conserved arginine is essential for nucleotide binding and hydrolysis $[121,122]$. In many cases AAA + ATPases act on a large number of substrate proteins, such as the general protein disaggregation machine Hsp104/ClpB or the motor of the bacterial degradation machinery ClpX. Generally, 
$\mathrm{AAA}+$ proteins form homohexameric arrangements with a central pore, stabilised by the presence of nucleotides. The AAA + modules of class I proteins therefore form two stacked hexameric rings [126, 127], while class II proteins form single hexameric ring $[118,128]$.

To date, three plant Rca crystal structures have been published. A $3.0 \AA$ model of the tobacco AAA+ domain (residues $68-360$ out of 383 total) [129], a $1.9 \AA$ model of the creosote bush C-terminal small domain (residues 250-351 out of 379 total) [130] and a $2.9 \AA$ model of the arabidopsis Rca (residues $65-362$ out of 416 total) [131]. These structures reveal the organisation of the apo state and architecture of the AAA+ domain.

Fig. 1.8B shows the crystal structure of Rubisco activase from tobacco in apo state [129]. The structure represents a typical AAA+ topology with large AAA+ domain comprising the nucleotide binding $\alpha / \beta$ subdomain and small $\alpha$-helical domain characterized by a 4 helix bundle. Rca also comprises the N-terminal domain (67 residues) and a C-terminal extension (23 residues), which were not resolved in the structure due to flexibility. Also the four flexible loop regions were not resolved in the structure and were extrapolated using dotted lines. The $\alpha / \beta$ subdomain has a Rossmann-fold typical for the AAA+ proteins, which constitute key signature motifs like Walker A, B and sensor 1 region essential for ATPase function [117]. The small C-terminal domain with 4-helix bundle ( $\alpha 6-10)$ fold at its core differs from the typical AAA + fold as helix 8 is elongated followed by small helical insertion $(\alpha 9)$ responsible for substrate specificity between Rubisco of the Solanaceae and nonSolanaceae family. Helix 9 is further connected to helix 10 through a linker (16 residues), that is totally separated from the main $\alpha$-helical sub-domain. The helix 8 in the structure of C-terminal of creosote bush is longer and consists of specificity domain. These models suggested a conformational plasticity in the specificitydetermining region that was responsible for differentiation between solanaceous (eg. tobacco) and non- solanaceous (eg. spinach) Rubiscos [47, 132]. 
A

\begin{tabular}{|c|c|c|c|}
\hline & & 252 & 360 \\
\hline $\begin{array}{c}\text { N-terminal } \\
\text { domain }\end{array}$ & AAA+ NBD & & \\
\hline
\end{tabular}

B
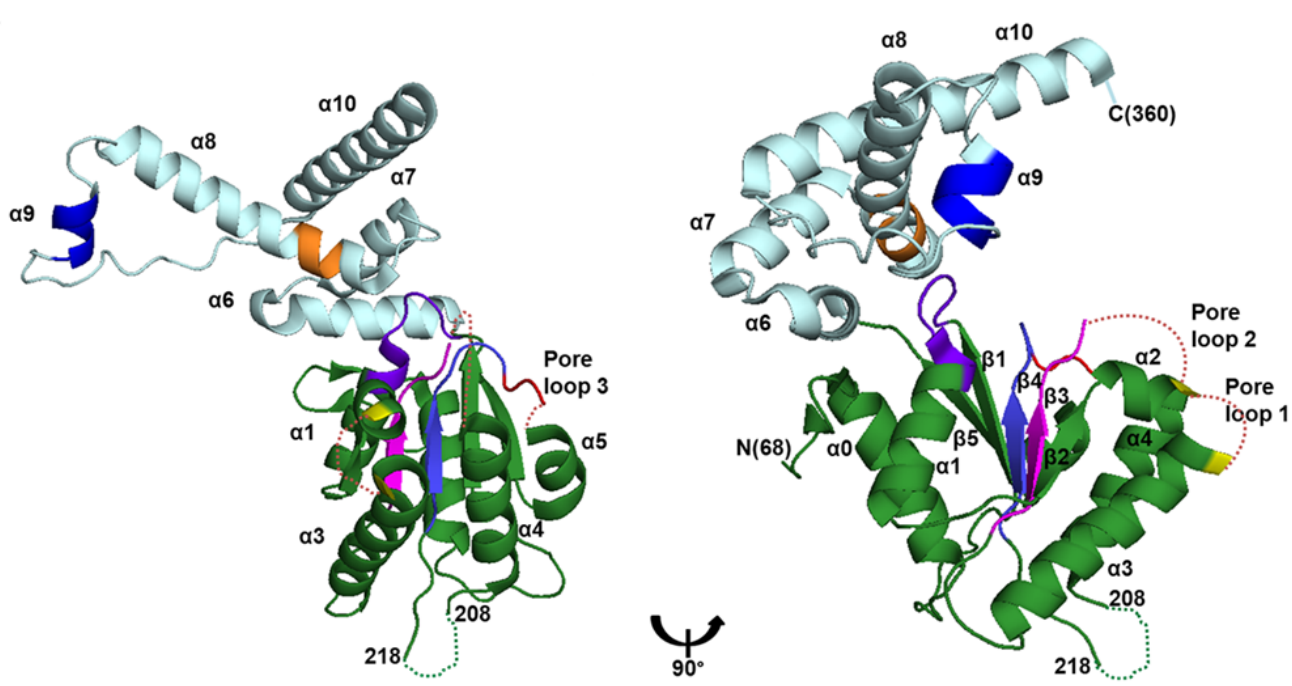

C

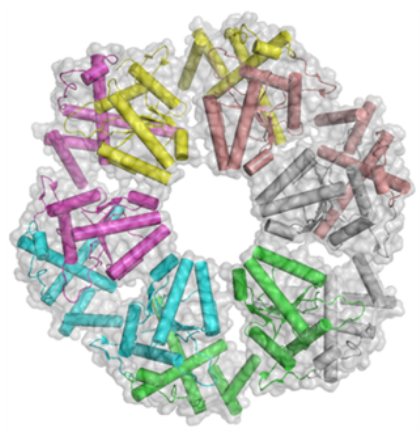

Top

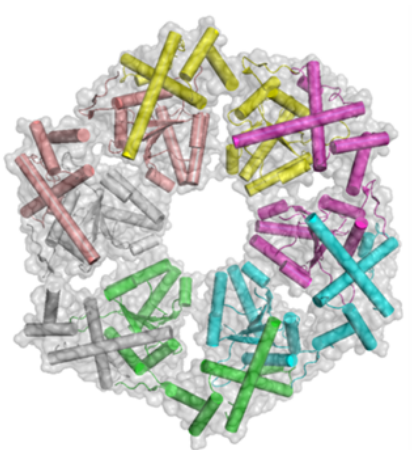

Bottom

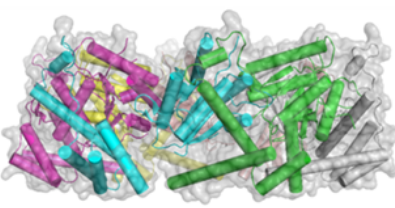

Side

Fig. 1.8: Structure and domain architecture of Rca from Nicotiana tabacum. (A). Domain architecture of tobacco activase (NtRca) showing the N-terminal domain, AAA+ nucleotide binding $\alpha / \beta$ sub-domain, $\alpha$ helical small subdomain $(\mathrm{hb})$ and $\mathrm{C}$-terminal extension. NBD: nucleotide binding domain, hb: $\alpha$-helical subdomain. (B) Ribbon representation of the crystal structure of NtRca (3T15). Disordered loops are indicated by dotted lines. Two views related by $90^{\circ}$ are shown. The $\alpha / \beta$ and the $\alpha-$ helical subdomains are indicated in pale cyan and green, respectively. The canonical AAA+ structural motifs are indicated as follows: Walker A (purple), Walker B (magenta), sensor I (tv blue) and sensor II (orange). The disordered pore loops are indicated as light red dots. The specificity helix (H9) is shown in blue. Another disordered loop (residue 208-218) and chain termini are indicated. (C) A hexameric model of NtRca (3ZW6).Top, bottom and side view of the surface of hexameric ring is shown, overlaid by the cylindrical representation. Each subunit is shown in different colour. The images were generated using pymol software. 


\subsubsection{Oligomeric state of the Rubisco activase}

The structural organisation of Rubisco activase oligomers has been a controversial issue. The published crystal structures do not provide a true functional oligomeric form of Rca.

Rubisco activase from diverse species are variedly polydisperse in nature. The oligomeric state of Rca in solution was observed to be varied in a concentration and nucleotide dependent manner. Various oligomeric sizes (50 600 kDa) from 1-16 subunits were reported, with larger species observed at higher enzyme concentrations [133-135]. The specific ATPase, Rubisco activation activity, and the oligomeric size increased with increasing concentration and in presence of macromolecular crowding agents such as polyethylene glycol [136].

It has been reported that a complex of no more than two to four subunits is required for biological activity of tobacco Rca [135] in a study using small angle x-ray scattering (SAXS) and analytical ultracentrifugation. However, this study was conducted in the absence of nucleotides under various dilutions. It has been hypothesized that Rca may form open spiral structures in solution [135, 137, 138] rather than a closed hexamer. It has also been interpreted that a large oligomer of more than eight subunits and up to sixteen subunits is the active state of activase [133, 134, 139]. However, this appears inconsistent with the canonical active functional oligomeric form (hexamer) of an AAA+ protein [120]. Therefore, it is possible that transient hexamers may exist in solution in the presence of nucleotide, however formation of hexamers and their dissociation is too rapid to be detected by the reported methods and therefore a range of oligomers were observed.

Biochemical analysis of interface mutants in tobacco revealed 2.9-3.7 and 3.5-5.4 as minimal subunits necessary for ATPase and Rubisco activation activity respectively [129] providing evidence for the hexamer as minimal functional unit. Indeed mutating the arginine 294 (R294) residue of tobacco Rca resulted in the formation of stable hexamers in the presence of $\mathrm{Mg}$.ATP $\gamma \mathrm{S}$ as observed by mass spectroscopy [140], negative stain EM [129], and analytical ultracentrifugation [141]. The biochemical analysis of this variant (R294V) revealed it to be fully functional, further providing a 
strong evidence in support of the hexamer as active species. Also, recently the $\alpha$ isoform and an equimolar mixture of $\alpha$ and $\beta$ isoform of spinach was observed to form a stable hexamer in presence of $\mathrm{Mg}$. ATP $\gamma \mathrm{S}$ as the first instance for any wild type Rca [141]. The non-homologous bacterial red-type activase CbbX was observed to change its oligomeric form in response to the presence of an allosteric regulator [114]. Thus it is quite possible that activase may acquire variable oligomeric states in solution but forms transient hexamers as an active species in presence of nucleotide, especially when engaging inhibited substrate Rubisco.

\subsubsection{Regulation of Rubisco activase}

In most plants, including arabidopsis, spinach, and rice [142-144], Rubisco activase occurs as two isoforms often as a result of alternative splicing of a single transcript. Consequently, the isoforms are essentially identical with the $\alpha$ isoform having a $\sim 40$ residue $\mathrm{C}$-terminal extension, which contains two conserved cysteine residues that are essential for its regulation $[145,146]$. However, in some plants like cotton, two isoforms are generated by different genes rather than splicing [147]. The activase is constitutively ATPase active and in contrast to many other AAA+ proteins the ATPase activity of activase is not stimulated by its protein substrate [109].

The ATPase activity of Rubisco activase is inhibited by ADP and regulated by the ATP/ADP ratio in the chloroplast stroma (Fig. 1.9A). This modulation of ATPase activity of activase also regulates Rubisco activity in response to light fluctuations $[113,148]$. It has been shown that the $\alpha$ isoform is more sensitive to ADP inhibition than the $\beta$ isoform $[146,148]$.

The $\alpha$ isoform is also regulated by changes in the redox state of two cysteine residues located at its C-terminal extension by thioredoxin- $\mathrm{f}$ [146]. Thioredoxins are a class of small redox proteins involved in several regulatory processes in plants [149]. Apart from Rubisco activase, thioredoxin-f regulates the activity of several other CalvinBenson cycle enzymes such as fructose 1,6-bisphosphatase, sedoheptulose 1,7bisphosphatase, phosphoribulokinase, and glyceraldehydes 3-phosphate dehydrogenase $[149,150]$. Trx- $f$ is reduced in the light via ferredoxin and ferredoxinthioredoxin reductase (FTR) using electrons originating from the action of 
photosystem I [151]. Reduced thioredoxin-f then diffuses throughout the stroma and reduces oxidized target proteins in the light leading to their activation (Fig. 1.9B).

In vitro, the redox regulation of Rca can be mediated by thioredoxin-f, along with a DTT electron source, which makes the ATP/ADP ratio much less inhibitory, causing an increase in ATPase and Rubisco activation activities of the $\alpha$ isoform. A similar effect was not observed in the presence of DTT and thioredoxin-m. This effect is reversible when DTT is replaced by oxidized glutathione leading to a reduction in activity [146]. Substituting either of these two cysteine residues from the C-terminal extension to alanine reduces the ATP/ADP sensitivity of the activase and the light responsiveness of Rubisco activity in vivo and in vitro [146, 152].

A

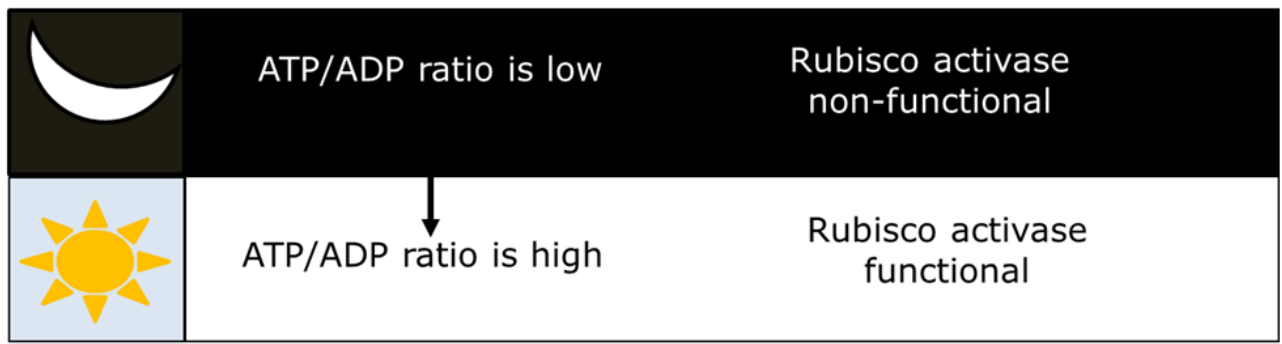

B

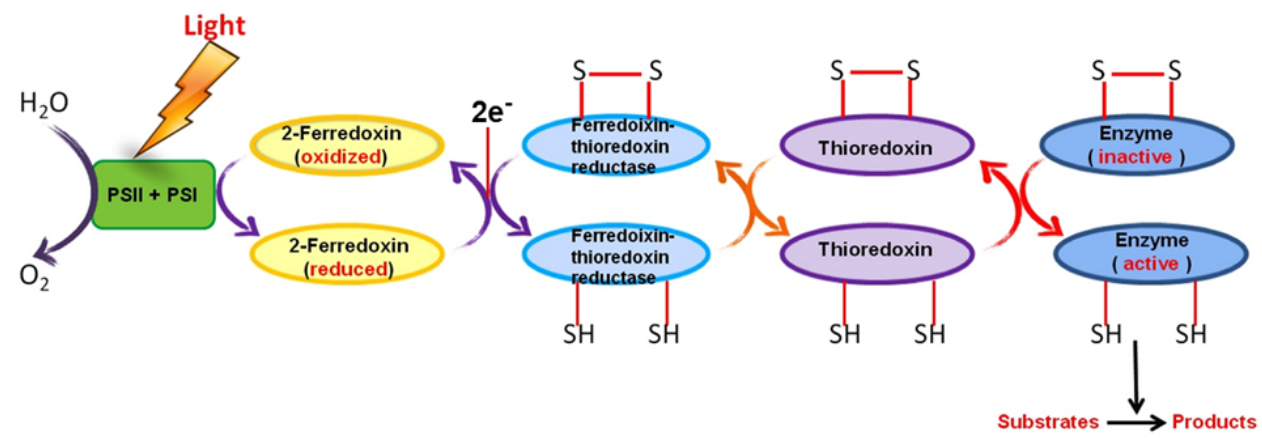

Fig.1.9: Regulation of Rubisco activase (A) By ATP/ADP ratio. During the day when the light reactions generate $\mathrm{ATP}$, the $\mathrm{ATP} / \mathrm{ADP}$ ratio is high, which activates activase. In the dark when ATP/ADP ratio is low activase becomes inactive due to inhibition by ADP. (B) By ferrodoxinthioredoxin system. Ferrodoxin is reduced by the photosynthetic electron transport chain. The reducing equivalent generated along with ferrodoxin reduces the disulphide group in the iron-sulphur enzyme ferrodoxin reductase. The reduced ferrodoxin reductase reduces the disulfide bond in thioredoxin. The reduced thioredoxin then reduces the disulfide bond of target enzymes to activate them. The activated enzyme gets deactivated by the oxidation thiol groups of reduced thioredoxin in the dark which in turn oxidizes the reduced enzyme. Modified from [153]. 
Although the $\alpha$ isoform is critical for redox regulation and important in regulating Rubisco activation in response to fluctuating light irradiance, interestingly some species like tobacco lack an $\alpha$ isoform. Plants expressing only the $\beta$ isoform did not show any downregulation in Rubisco activity during a light-dark transition [152] and the $\beta$ isoform was not observed to be redox sensitive when assayed alone. The regulation of $\beta$ isoform by the ADP/ATP ratio varies among different species. For instance, the $\beta$ isoforms of tobacco is reportedly more prone to inhibition by ADP than the $\beta$ isoform of arabidopsis [154]. Subunit mixing experiments between $\alpha$ and $\beta$ isoforms under in vitro assay conditions validated a regulatory effect of the $\alpha$ isoform on the $\beta$ isoform in arabidopsis. At the equimolar ratio, the $\beta$ isoform gets totally inhibited by the presence of $\alpha$ isoform at an ATP/ADP ratio of 1:3 under oxidizing conditions [155], indicating that the $\alpha$ isoform allosterically modulates the activity of $\beta$ isoforms.

Under in vivo conditions, reducing equivalents increase with light illumination, hence it was assumed that redox chemistry of the $\alpha$ isoform modulates activase activity in response to light and regulates Rubisco activity. Later it was confirmed by a study expressing just a single isoform in vivo. Arabidopsis rca mutants expressing only the $\alpha$ isoform respond to light fluctuations and Rubisco activation was downregulated under light-limiting condition, while the expression of just the $\beta$ isoform did not downregulate Rubisco activity in response to light [152]. Based on the mutational and cross-linking study, the C-terminal extension of the $\alpha$ isoform in the oxidized state folds back towards the nucleotide binding pocket, perturbing its activity $[155,156]$. Furthermore, there is fluctuation in the diurnal expression of activase in plants with maximum expression at the onset of the photocycle and lowest at night [157], which closely mimics the circadian rhythm of Rubisco activation.

\subsubsection{Mutational studies on Rubisco activase}

Multiple mutational studies have been performed in the past to elucidate the determinants of activase function, long prior to structural information becoming available. Based on this body of work, some critical residues involved in ATPase and activase function were identified by mutational and biochemical analysis and were consistent with AAA+ domain architecture. Fig. 1.10 provides an overview of the prior mutational studies conducted on Rubisco activase and highlights residues 
responsible for particular functions. The work has been reviewed by Portis (2003) and Stotz et al. (2011).

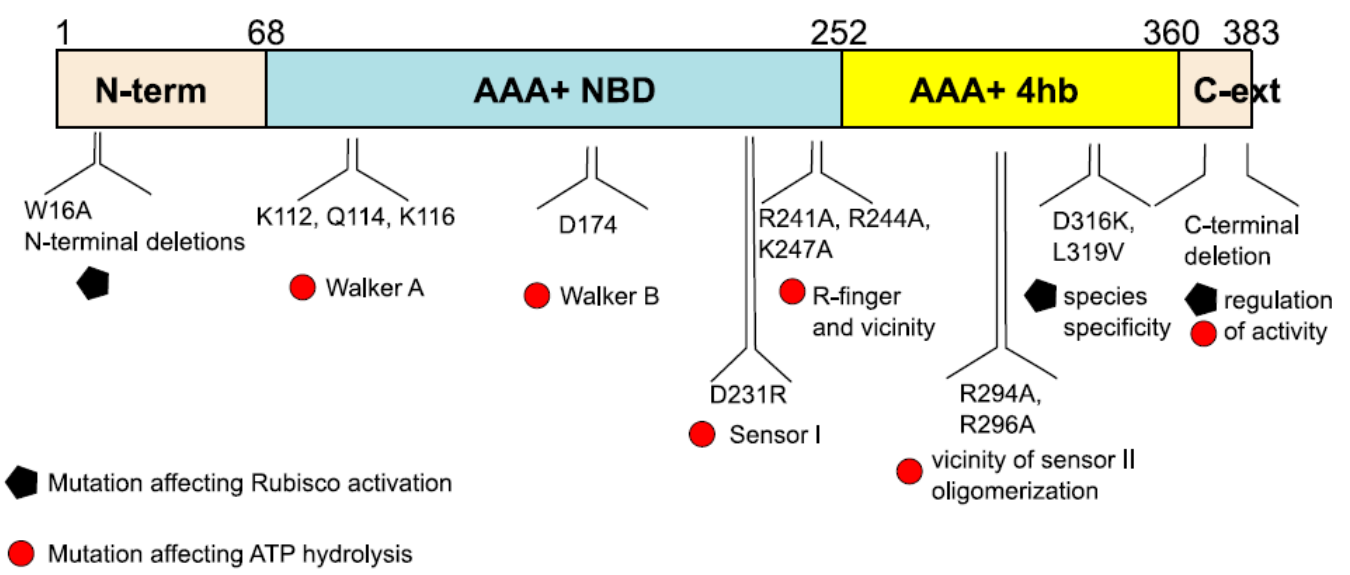

Fig. 1.10: Domain architecture of Rubisco activase with a bird eye view of mutational studies at specific region. Specific regions are mentioned (N-term: N-terminal domain. NBD: nucleotide binding $\alpha / \beta$-subdomain. 4-hb: 4-helix bundle subdomain. C-ext: C-terminal extension). Residues are highlighted (tobacco numbering) with their specific effect. Reproduced from [158].

In many $\mathrm{AAA}+$ proteins, the $\mathrm{N}$-terminal domain acts as an adaptor for engaging substrate proteins. Consistently, mutations and truncations of the N-terminal region had little effect on ATPase activity but critically impaired Rubisco activation [159, 160]. The conserved tryptophan (16) residue was critically implicated in Rubisco activation in tobacco and spinach. The deletion of residues upstream of this tryptophan residue preserved the activation function, while in spinach, truncating just the initial 12 (W11 in spinach) residues led to loss of activation function [159, 160]. Furthermore, deletion of the initial 50 residues at the N-terminal domain led to no loss of ATPase function but almost complete loss of Rubisco activation without any observed increase in intrinsic fluorescence [159]. This suggests that the N-terminal domain is associated with Rubisco activation alone and not with ATPase function.

Critical residues were identified to be crucial for ATPase activity in the NBD even before Rca was classified as an AAA+ protein. The residues identified, belong to the featured motifs of typical AAA+ proteins and are important for maintaining ATPase function. For instance, lysine (116) at the Walker A motif was implicated in nucleotide binding using photo-affinity labelling experiments with nucleotide 
analogues $[148,161]$. Other residues including glutamine (114) and lysine (112) were also found to be important for ATPase activity [148, 162]. The sensor 1 region and Walker B motif was suggested to be involved in nucleotide hydrolysis and in coordination of ATP [163], while arginine finger $(241,244)$ and a lysine $(247)$ in its vicinity were responsible for intersubunit communication or oligomerization and nucleotide hydrolysis $[164,165]$. The mutations of glutamine (114) to aspartate or glutamate led to a stimulation of ATPase activity and enhanced activation. The acidic substitution mutant showed less inhibition by ADP suggesting its involvement in ADP inhibition $[162,166]$.

Importantly, the two amino acid substitutions (D316K and L319V) targeted at a small helical insertion in the C-terminal 4-helix bundle domain were found to confer Rubisco specificity between Solanaceae and non-Solanaceae plant family [132]. The corresponding interaction partner residues R/P89 and K/D94 (spinach numbering) were later found in Rubisco $[167,168]$. Since the residue substitutions involved in specificity reversibility were charge switched mutations, a direct physical interaction was suggested between Rubisco and Rca due to formation of a salt bridge between these residues [132].

The two conserved arginine residues in the vicinity of sensor 2 have been found to be important for ATP hydrolysis. Mutation of arginine to alanine (R294A/R296A) led to reduced ATPase and activation activity [165]. Mutation of R294 also affects oligomerization and another variant of this mutant (R294V) in tobacco was observed to form stable hexamers in presence of $\mathrm{Mg} \cdot \mathrm{ATP} \gamma \mathrm{S}$ and possessed a WT-like biochemical phenotype [140, 141, 158].

The C-terminal extension of plant Rca is less conserved as compared to central AAA+ subdomains and varies in length in the two Rca isoforms ( $\alpha$ and $\beta$ ) in many species. Mutation of the conserved tyrosine (Y361A) completely abolished ATPase and activation activity in tobacco [129]. Truncation of the $\mathrm{C}$-terminal $(\Delta \mathrm{C} 363)$ reduced ATPase activity but stimulated activation rate, while another truncation $(\Delta C 360)$ led to a total loss of ATPase and activase functions indicating the role of conserved tyrosine in regulation $[129,160]$. Furthermore, mutation of two conserved cysteines in the extended C-terminus of the $\alpha$ isoform reduced its sensitivity towards ADP inhibition suggesting the role of C-terminus in redox regulation [146]. The role of the 
flexible $\mathrm{C}$-terminus in allosteric regulation has been demonstrated for other AAA+ protein spastin [128].

\subsubsection{The role of Rubisco activase in regulating photosynthesis at elevated temperatures}

Since Rubisco activase is critically responsible for maintaining the activation state of Rubisco in vivo, the mechanism and regulation of Rca are the focus of current research. However, Rca is a thermolabile enzyme and inactivation of Rca often limits photosynthetic efficiency at moderately elevated temperatures [11]. Therefore, activase with improved thermal stability is believed to be result in plants with improved photosynthetic performance and growth phenotypes under moderate heat stress $[12,18,169]$. This hypothesis has been strongly supported by expressing more thermostable variants of Rca in arabidopsis, which resulted in plants with higher rate of photosynthesis and growth at elevated temperature $[12,13]$.

It has also been argued that deactivation of Rubisco at elevated temperature may be a regulatory response to protect the plant during heat stress $[16,17,170]$. Photosystem II was considered as the major cause of thermal damage in the past [171] however, PS II was reported to be unaffected at temperature that inhibits $\mathrm{CO}_{2}$ fixation $[172,173]$. Other electron transport limitations such as RuBP regeneration, stability of the thylakoid membrane and ATP/ADP levels were also supposed to constrain photosynthesis at high temperature $[16,174,175]$. Also, the solubility of $\mathrm{CO}_{2}$ and Rubisco specificity $\left(\mathrm{S}_{\mathrm{c} / \mathrm{o}}\right)$ reduces at high temperature favouring the oxygenation reaction leading to production of more misfire products $[99,176]$. Therefore, it was hypothesized that activase may function as a fuse to reduce Calvin-Benson cycle flux as a regulatory mechanism to protect Rubisco and the photosynthetic apparatus at high temperature [16].

It has been mentioned that conformational flexibility of Rca is required for it to remodel Rubisco conformation and this likely be the reason why Rca cannot be thermostable [15]. Furthermore, research comparing a warm-season plant and a cool season plant also showed that there was a trade-off between Rca thermostability and its activity at low temperature [15, 154, 169]. The analysis of different genetic variants of rice yielded similar results where a rice species (Oryza australiensis) 
endemic to hot and arid zone displayed higher thermotolerance than the crop species (Oryza sativa) at the cost of catalytic activity [177].

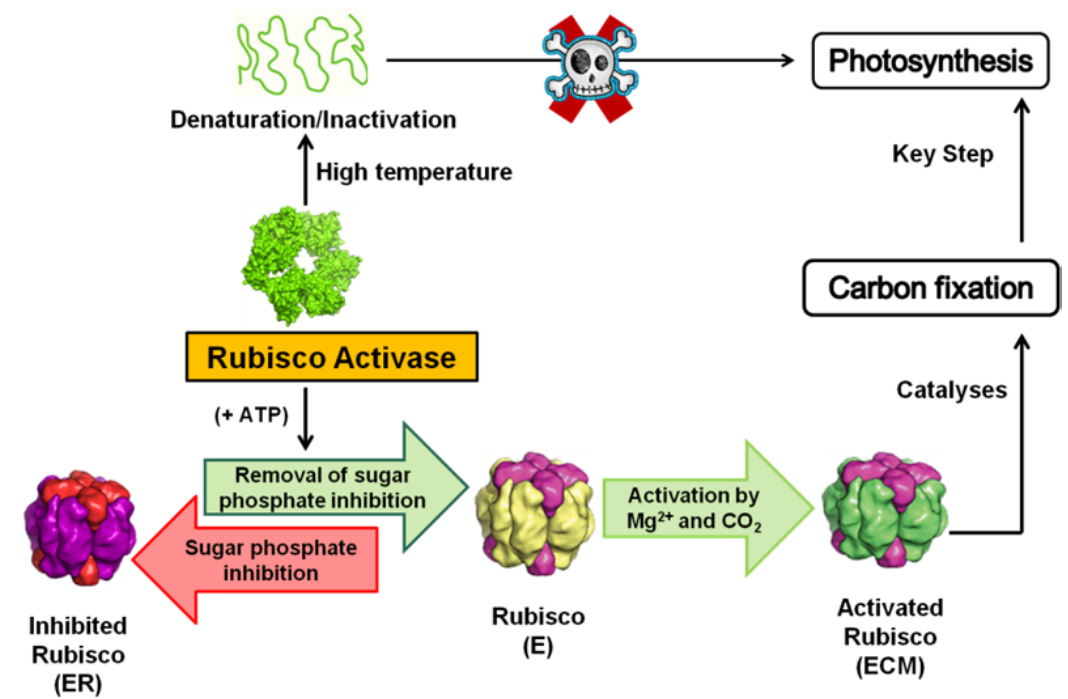

Fig. 1.11: Activase based model of thermal inhibition of photosynthesis at high temperature. At high temperature, activase denatures and thus fails to maintain the activation state of Rubisco, impairing photosynthesis.

The stability of Rca varies between different species, with $\alpha$ isoform reported to be more thermostable than the $\beta$ isoform in spinach but not in cotton and arabidopsis $[147,166]$ where both isoforms have similar thermostability. In spinach, the $\alpha$ isoform also confers its thermostability to the $\beta$ isoform $[141,178]$. Rca shows enhanced thermostability in presence of nucleotide, with ADP and ATP guarding the $\beta$ isoform of arabidopsis and cotton against heat inactivation $[134,138]$. Improved thermotolerance of the $\alpha$ and the $\beta$ isoform was observed in spinach in presence of ADP, while the $\alpha$ isoform of spinach showed a dramatic increase in its thermal stability in presence of ATP $\gamma \mathrm{S}[141,178]$. The molecular mechanism underlying the thermolability of photosynthesis is of particular pertinence due to the predicted effects of global climate change [18]. Further insights from structural and biochemical work may generate the possibility to discover and engineer more thermostable variants. 


\subsection{Aim and scope of the study}

Rubisco activase is critical for maintenance and regulation of photosynthesis in plants. However, the thermolability of activase limits its ability to perform its function at supraoptimal temperature. It has been suggested that improving activase properties would result in plants with improved photosynthetic properties and growth $[10,12$, 18]. The mechanism of this AAA+ remodelling enzyme has remained elusive in spite of recently available incomplete three dimensional crystal structures [129-131]. The aim of the current study therefore was to take advantage of newly gained structural and biochemical understanding to obtain a comprehensive mechanistic view of the behaviour of this agriculturally relevant Rubisco activation system in the key crop plant Oryza sativa (rice). Additionally, characterization of a Rca homologue from a challenging desert environment was undertaken to explore the possibility of discovering naturally thermostable plant Rubisco activases. This study aimed to generate a comprehensive molecular toolbox to address pertinent questions in plant Rubisco regulation from a biochemical angle. In long term, we aim to build a solid biochemical foundation for the next wave of crop improvement technologies, targeting at enhancing efficiency of photosynthesis to improve crop yield. 
2. Materials and Methods 


\subsection{Chemicals}

All the chemicals used in the study were of at least analytical grade. Chemicals other than the ones listed below were purchased from Sigma-Aldrich:

\begin{tabular}{|c|c|}
\hline Reagent & Supplier \\
\hline Glycerol & \multirow{3}{*}{ Affymetrix } \\
\hline Sodium phosphate, monobasic, $98 \%$, ACS reagent grade & \\
\hline Yeast extract & \\
\hline Coomassie brilliant blue, G-250 & Applied Chemistry \\
\hline Bacto agar & BD, Difco \\
\hline Tryptone & BD, Difco \\
\hline $30 \%$ Acryl/ bis solution $37.5: 1$ & \multirow{5}{*}{ Bio-Rad } \\
\hline EDTA disodium salt di-hydrate & \\
\hline Ethidium bromide (10 mg/ml Sol.) & \\
\hline TEMED & \\
\hline Tris & \\
\hline dNTP Set $100 \mathrm{mM}$ solutions & \multirow{2}{*}{ Fermentas } \\
\hline X-Gal & \\
\hline Bleach $5 x$ concentrate & GB bleach \\
\hline Kanamycin sulphate & Gibco \\
\hline Ampicillin & \multirow{3}{*}{ Gold Biotechnology } \\
\hline Chloramphenicol & \\
\hline IPTG & \\
\hline SDS, Sodium dodecyl sulphate & Hoefer \\
\hline SYBR Safe ${ }^{\circledR}$ DNA gel stain & Invitrogen \\
\hline Acetic acid (Glacial) $100 \%$ anhydrous & \multirow{6}{*}{ Merck } \\
\hline Ethanol 99.5-99.8\%, absolute, GR grade for analysis & \\
\hline Methanol, GR, $\min 99.8 \%$ & \\
\hline $\mathrm{N}, \mathrm{N}$-Dimethyl formamide AR & \\
\hline Sodium chloride & \\
\hline Sodium hydroxide, analysis grade pellets, $99 \%$ & \\
\hline Magnesium chloride hexahydrate & MP Biomedicals \\
\hline
\end{tabular}




\begin{tabular}{|c|c|}
\hline Surface disinfectant & Pharmacon-Virkon \\
\hline Ammonium persulphate (molecular grade) & \multirow{2}{*}{ Promega } \\
\hline Proteinase $\mathrm{K}$ & \\
\hline Phosphoenolpyruvate (PEP) & \multirow{2}{*}{ Roche } \\
\hline Creatine Kinase & \\
\hline DTT, Cleland's Reagent & \multirow{2}{*}{ Thermo Scientific } \\
\hline HisPur $^{\mathrm{TM}}$ Ni-NTA resin & \\
\hline Agarose (molecular grade) & Vivantis \\
\hline Polyethylene glycol (PEG) 3350 20\% (v/v) & Sigma \\
\hline
\end{tabular}

Table 2.1: Chemicals

\subsection{Enzymes and kits}

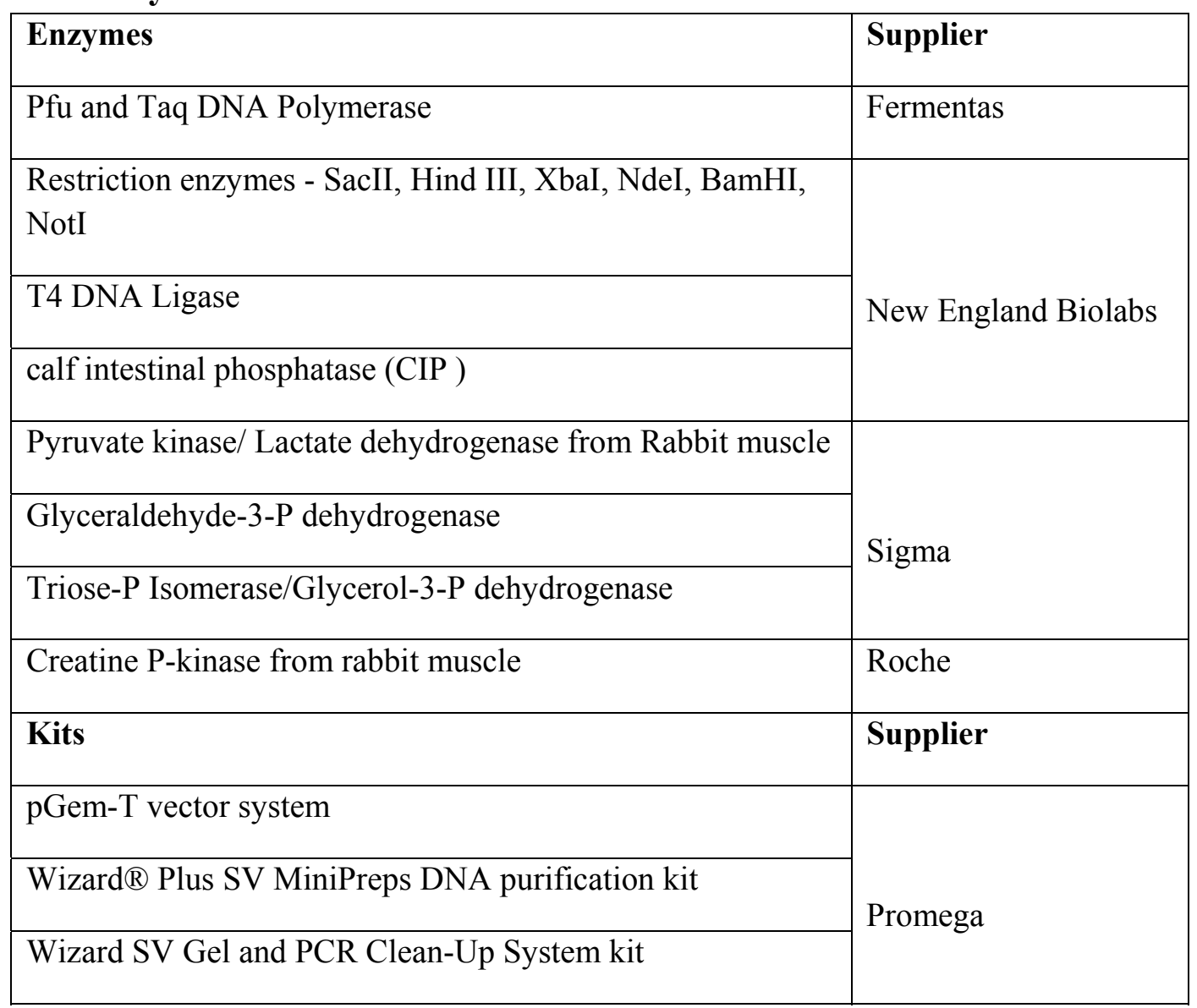

Table 2.2: Enzymes and kits 


\subsection{Instruments}

\begin{tabular}{|c|c|}
\hline Instruments & Supplier \\
\hline TB2 Thermo block & \multirow{2}{*}{ BioMetra } \\
\hline Professional TRIO thermocycler & \\
\hline Mini-protean tetra system & \multirow{3}{*}{ Bio-Rad } \\
\hline Power pac Basic & \\
\hline Power pac $\mathrm{HC}$ & \\
\hline Thermo mixer comfort & Eppendorf \\
\hline PL-600 pH/mV/Temp meter & Ezodo \\
\hline Akta Purifier Frac900, Akta micro & \multirow{4}{*}{ GE health care } \\
\hline Superdex 200 16/600, 10/300, 3.2/30 & \\
\hline MonoQ HR 10/100 & \\
\hline SourceQ 15 and 30 & \\
\hline Minicentrifuge & Green liv \\
\hline Z300K refrigirated Bench top centrifuge & Hermle \\
\hline Safe imager 2.0 & \multirow{3}{*}{ Invitrogen } \\
\hline Trans-Blot & \\
\hline Semi dry Transfer cell & \\
\hline Bench top Microcentrifuge & Kintaro \\
\hline Excella E25 Incubater & \multirow{2}{*}{ New Brunswick } \\
\hline Innovo 44R & \\
\hline Rocker 400 & Rocker \\
\hline Vortex genie2 & Scientific industries \\
\hline UV-1800 spectophotometer & \multirow{2}{*}{ Shimadzu } \\
\hline Cps controller & \\
\hline Sorval Micro centrifuge & \multirow{5}{*}{ Thermo Scientific } \\
\hline Nanodrop 2000 & \\
\hline Forma $900\left(-80^{\circ} \mathrm{C}\right)$ & \\
\hline MaxQ 6000 incubator & \\
\hline Cimarec strirrer & \\
\hline Sonics Sonicator & Vibra cell \\
\hline
\end{tabular}

Table 2.3: Instruments 


\subsection{Buffers and media}

Media were prepared with deionised, double-distilled water and subsequently autoclaved.

LB medium:

- $\quad 10 \mathrm{~g} / 1$ Tryptone

- $5 \mathrm{~g} / \mathrm{l}$ Yeast extract

- $10 \mathrm{~g} / \mathrm{l} \mathrm{NaCl}$

- $\quad$ (15 g/l agar for solid medium)

- $\quad$ pH 7.1 adjusted with $\mathrm{NaOH}$

\subsubsection{Buffers composition}

\begin{tabular}{|c|c|}
\hline Buffers & Composition \\
\hline Coomassie destaining solution & $10 \%$ ethanol, $7 \%$ acetic acid \\
\hline Coomassie staining solution & $\begin{array}{l}0.1 \%(\mathrm{w} / \mathrm{v}) \text { Serva Coomassie Blue R-250, } 40 \% \\
\text { ethanol, } 8 \% \text { acetic acid }\end{array}$ \\
\hline $5 \mathrm{xDNA}$ loading dye & $\begin{array}{l}0.1 \mathrm{M} \text { EDTA, } 0.1 \%(\mathrm{w} / \mathrm{v}) \mathrm{SDS}, 0.025 \%(\mathrm{w} / \mathrm{v}) \text { bromo } \\
\text { phenol blue, } 40 \% \text { glycerol }\end{array}$ \\
\hline $\begin{array}{l}\text { Electrophoresis migration } \\
\text { buffer }\end{array}$ & $\begin{array}{l}120 \mathrm{mM} \text { Tris- } \mathrm{HCl}, 134 \mathrm{mM} \text { glycine, } \\
0.1 \% \text { SDS }\end{array}$ \\
\hline TAE & $40 \mathrm{mM}$ Tris-acetate, $\mathrm{pH} 8.5,2 \mathrm{mM}$ EDTA \\
\hline TBS & $50 \mathrm{mM}$ Tris- $\mathrm{HCl} \mathrm{pH} 8.0,137 \mathrm{mM} \mathrm{NaCl}, 2.7 \mathrm{mM} \mathrm{KCl}$ \\
\hline TBS-T & $\begin{array}{l}50 \mathrm{mM} \text { Tris- } \mathrm{HCl} \mathrm{pH} 8.0,137 \mathrm{mM} \mathrm{NaCl}, 2.7 \mathrm{mM} \mathrm{KCl} \text {, } \\
0.1 \% \text { Tween } 20\end{array}$ \\
\hline PonceauS staining solution & $0.1 \%(\mathrm{w} / \mathrm{v})$ PonceauS, $5 \%$ aceticacid \\
\hline Lysis buffer A & $\begin{array}{l}50 \mathrm{mM} \text { Tris- } \mathrm{HCl} \mathrm{pH} 8.0,50 \mathrm{mM} \mathrm{NaCl}, 10 \mathrm{mM} \\
\text { imidazole }\end{array}$ \\
\hline Lysis buffer B (Elution buffer) & $\begin{array}{l}50 \mathrm{mM} \text { Tris- } \mathrm{HCl} \mathrm{pH} 8.0,50 \mathrm{mM} \mathrm{NaCl}, 200 \mathrm{mM} \\
\text { imidazole }\end{array}$ \\
\hline Purification buffer $\mathrm{A}$ & $20 \mathrm{mM}$ Tris- $\mathrm{HCl} \mathrm{pH} 8.0,50 \mathrm{mM} \mathrm{NaCl}$ \\
\hline Purification buffer B & 20 mM Tris- $\mathrm{HCl} \mathrm{pH} 8.0,1 \mathrm{M} \mathrm{NaCl}$ \\
\hline Rubisco extraction buffer & $\begin{array}{l}50 \mathrm{mM} \text { Tris- } \mathrm{HCl} \mathrm{pH} 8.0,50 \mathrm{mM} \mathrm{NaCl}, 1 \mathrm{mM} \\
\mathrm{Na}_{2} \text { EDTA, } 5 \mathrm{mM} \text { DTT, } 1 \mathrm{mM} \text { PMSF, } 12.5 \% \text { glycerol, }\end{array}$ \\
\hline
\end{tabular}




\begin{tabular}{|c|c|}
\hline & $2 \%$ PVPP \\
\hline Rubisco resuspension buffer & $\begin{array}{l}50 \mathrm{mM} \text { Tris-HCl pH 8.0, } 50 \mathrm{mM} \mathrm{NaCl}, 1 \mathrm{mM} \\
\mathrm{Na}_{2} \text { EDTA, } 5 \mathrm{mM} \text { DTT, } 12.5 \% \text { glycerol }\end{array}$ \\
\hline Rubisco purification buffer A & $\begin{array}{l}\text { Purification Buffer } \mathrm{A}+0.1 \mathrm{mM} \mathrm{Na} \mathrm{ND}_{2} \mathrm{ETA}, 1 \mathrm{mM} \\
\text { DTT, } 12.5 \% \text { glycerol }\end{array}$ \\
\hline Rubisco purification buffer B & $\begin{array}{l}\text { Purification Buffer } \mathrm{B}+0.1 \mathrm{mM} \mathrm{Na} \mathrm{N}_{2} \mathrm{EDTA}, 1 \mathrm{mM} \\
\text { DTT, } 12.5 \% \text { glycerol }\end{array}$ \\
\hline Rubisco dialysis buffer & Same as Rubisco purification Buffer A \\
\hline 5x SDS-sample buffer & $\begin{array}{l}225 \mathrm{mM} \text { Tris- } \mathrm{HCl} \mathrm{pH} 6.8,50 \% \text { glycerol } \\
5 \% \mathrm{SDS}(\mathrm{w} / \mathrm{v}), 5 \% \text {-mercaptoethanol } \\
0.01 \% \text { bromphenolblue (w/v) }\end{array}$ \\
\hline Western blot transfer buffer & $50 \mathrm{mM}$ Tris- $\mathrm{HCl}, 20 \%$ methanol, $192 \mathrm{mM}$ glycine \\
\hline
\end{tabular}

Table 2.4: Buffers Composition

Buffers used for protein purification were vacuum filtered and degassed.

\subsection{Oligonucleotides}

Oligonucleotides used in the study were purchased from IDT (Singapore, Singapore).

\begin{tabular}{|l|l|}
\hline Name & Sequence \\
\hline$\Delta$ N67OsRcaSacIIfor & CTCCGCGGTGGTGACTTCGACAACACC \\
\hline T7 Term & GCTAGTTATTGCTCAGCGG \\
\hline OsRcaW15Afor & CAGGACAGGGCGAAGGGTCTC \\
\hline OsRcaW15Arev & GAGACCCTTCGCCCTGTCCTG \\
\hline OsRcaS23Afor & GCCTACGACATCGCCGATGACCAGCA \\
\hline OsRcaSS23Arev & TGCTGGTCATCGGCGATGTCGTAGGC \\
\hline OsRcaS23Dfor & GCCTACGACATCGACGATGACCAGCAG \\
\hline OsRcaS23Drev & CTGCTGGTCATCGTCGATGTCGTAGGC \\
\hline OsRcaF68Lfor & ACGTACGACCTCGACAACACCATG \\
\hline OsRcaF68Lrev & CATGGTGTTGTCGAGGTCGTACGT \\
\hline OsRcaG73Dfor & GACAACACCATGGACGGCTTCTAC \\
\hline OsRcaG73Drev & GTAGAAGCCGTCCATGGTGTTGTC \\
\hline OsRcaS90Tfor & GTCGTCCACATCACCAAGAACTTCATG \\
\hline OsRcaS90Trev & CATGAAGTTCTTGGTGATGTGGACGAC \\
\hline
\end{tabular}




\begin{tabular}{|c|c|}
\hline OsRcaT95Nfor & AAGAACTTCATGAACCTCCCCAAC \\
\hline OsRcaT95Nrev & GTTGGGGAGGTTCATGAAGTTCTT \\
\hline OsRcaK115Afor & GGGTCAGGGAGCATCCTTCCAGT \\
\hline OsRcaK115Arev & ACTGGAAGGATGCTCCCTGACCC \\
\hline OsRcaA143Vfor & AGCGGCAACGTGGGAGAGCCG \\
\hline OsRcaA143Vrev & CGGCTCTCCCACGTTGCCGCT \\
\hline OsrcaG144A for & GGCAACGCCGCAGAGCCGGCG \\
\hline OsrcaG144A rev & CGCCGGCTCTGCGGCGTTGCC \\
\hline OsrcaE145A for & GGAGCGCCGGCGAAGCTGATC \\
\hline OsrcaE145A rev & GATCAGCTTCGCCGGCGCTCC \\
\hline OsRcaK148Afor & CCGGCGGCGCTGATCAGGCAG \\
\hline OsRcaK148Arev & CTGCCTGATCAGCGCCGCCGG \\
\hline OsRcaQ152Afor & CTGATCAGGGCGCGGTACCGT \\
\hline OsRcaQ152Arev & ACGGTACCGCGCCCTGATCAG \\
\hline OsRcaE156Afor & TACCGTGCGGCGGCAGACATC \\
\hline OsRcaE156Arev & GATGTCTGCCGCCGCACGGTA \\
\hline OsRcaD159Afor & GCGGCAGCCATCATCAAGAAGGGG \\
\hline OsRcaD159Arev & ССССTTCTTGATGATGGCTGCCGC \\
\hline OsRcaK162Afor & GCAGACATCATCGCGAAGGGGAAG \\
\hline OsRcaK162Arev & CTTCCCCTTCGCGATGATGTCTGC \\
\hline OsRcaD173Afor & CTTCATCAACGCTCTGGACGCGG \\
\hline OsRcaD173Arev & CCGCGTCCAGAGCGTTGATGAAG \\
\hline OsRcaY214Afor & CCCGGGATGGCCAACAAGGAGGAC \\
\hline OsRcaY214Arev & GTCCTCCTTGTTGGCCATCCCGGG \\
\hline OsRcaN215Afor & GGGATGTACGCCAAGGAGGACAAC \\
\hline OsRcaN215Arev & GTTGTCCTCCTTGGCGTACATCCC \\
\hline OSRcaK216Afor & ATGTACAACGCGGAGGACAACCCC \\
\hline OSRcaK216Arev & GGGGTTGTCCTCCGCGTTGTACAT \\
\hline OsRcaE217Qfor & TACAACAAGCAGGACAACCCCCGT \\
\hline OsRcaE217Qrev & ACGGGGGTTGTCCTGCTTGTTGTA \\
\hline OSRcaD218Afor & AACAAGGAGGCCAACCCCCGTGTC \\
\hline OSRcaD218Arev & GGGGTTGTCCTCCGCGTTGTACAT \\
\hline
\end{tabular}




\begin{tabular}{|c|c|}
\hline OSRcaN219A for & AAGGAGGACGCCCCCCGTGTC \\
\hline OSRcaN219Arev & GACACGGGGGGCGTCCTCCTT \\
\hline OsRcaP220Afor & AAGGAGGACAACGCCCGTGTC \\
\hline OsRcaP220Arev & GACACGGGCGTTGTCCTCCTT \\
\hline OsRcaR293Vfor & TTCGGCGCTCTTGTAGCCCGTGTTTAC \\
\hline OsRcaR293Vrev & GTAAACACGGGCTACAAGAGCGCCGAA \\
\hline OsRcaK315Dfor & AACATTGGCGACAGGCTGGTGAAC \\
\hline OsRcaK315Drev & GTTCACCAGCCTGTCGCCAATGTT \\
\hline OsRcaR321Afor & CTGGTGAACTCGGCGGAGGGCCCA \\
\hline OsRcaR321Arev & TGGGCCCTCCGCCGAGTTCACCAG \\
\hline OsTrx-fC42S for & TGGTGCGGCCCTTCCAAGGTGATGGCA \\
\hline OsTrx-fC42Srev & TGCCATCACCTTGGAAGGGCCGCACCA \\
\hline OsRca2 $\Delta 21 \_$SacIIfor & AACCGCGGTGGTGAGGAGGGGCG \\
\hline OsRca2 $\Delta 21$ NotIrev & TTGCGGCCGCTTAATCATCTAAATTCTT \\
\hline pUC57(-Apo1)for & ACGGCCAGTGAAATCGAGCTCGGTA \\
\hline pUC57(-Apo1)rev & TACCGAGCTCGATTTCACTGGCCGT \\
\hline OsRca $\beta(G 738 \mathrm{~A})$ for & GGGCGTATGGAGAAATTCTACTGGGCT \\
\hline OsRca $\beta(G 738 \mathrm{~A}) \mathrm{rev}$ & AGCCCAGTAGAATTTCTCCATACGCCC \\
\hline AtRca $\alpha$ G381_K382ins(S)for & AAATTTCTACGGATCCAAAGCGGCCCAG \\
\hline AtRcaaG381_K382ins(S)rev & CTGGGCCGCTTTGGATCCGTAGAAATTT \\
\hline OsRca $\alpha$ G381_Q382ins(S)for & TTCCTTCTACGGATCCCAAGGAGCACAG \\
\hline OsRca $\alpha$ G381_Q382ins(S)rev & CTGTGCTCCTTGGGATCCGTAGAAGGAA \\
\hline
\end{tabular}

Table 2.5: Primers used in this study

\subsection{Molecular biology methods}

\subsubsection{DNA analytical methods}

UV spectroscopy (Nanodrop 2000 (Thermo scientific)) was used to determine the DNA concentrations. The measurements were carried out at $260 \mathrm{~nm}$, where one absorption unit equals to $50 \mathrm{ng} / \mu \mathrm{l}$ of DNA. DNA samples were subjected to horizontal agarose gels electrophoresis for separation according to size. The DNA samples to be applied on the gel were mixed with 5X DNA loading dye. Agarose gel electrophoresis was performed in $1 \%(\mathrm{w} / \mathrm{v})$ TAE-agarose gels supplemented with 
SYBR-SAFE (1:10000) for DNA visualization in 1X TAE buffer. The gels were run at constant $100 \mathrm{~V}$ and DNA fragments were visualized by blue light (Invitrogen). Cloned constructs were verified using a restriction enzyme digest analysis with suitable restriction enzymes and DNA sequencing by 1st base (Singapore). For one sequencing reaction, $1 \mu \mathrm{g}$ of plasmid was needed. Sequencing results were analysed using the programs BioEdit (Ibis Biosciences, USA) and MultAlin (http://multalin.toulouse.inra.fr/multalin/) for comparative sequence alignment analysis.

\subsubsection{Competent $E$. coli cells preparation and transformation}

Chemically competent DH5 $\alpha$ and BL21 cells were prepared by the $\mathrm{CaCl}_{2}$ method [179]. Cells from glycerol stock were revived by streaking onto an agar plate and growing overnight $(8-16 \mathrm{~h})$ at $37^{\circ} \mathrm{C}$. A single colony was inoculated in LB media and grown overnight in INNOVA 44R incubator shaker (New Brunswick Scientific) at 37 ${ }^{\circ} \mathrm{C}, 220 \mathrm{rpm}$. The overnight grown culture was used to inoculate $11 \mathrm{of}$ media and grown to mid-log phase $\left(\mathrm{OD}_{600}=0.5\right)$ at $37^{\circ} \mathrm{C}, 220 \mathrm{rpm}$. After chilling the cells on ice for $20 \mathrm{~min}$, the culture was centrifuged for $30 \mathrm{~min}$ at $4000 \mathrm{rpm}$ at $4{ }^{\circ} \mathrm{C}$ and pelleted cells were resuspended gently in $20 \mathrm{ml}$ ice-cold $0.1 \mathrm{M} \mathrm{CaCl}_{2}, 15 \%$ glycerol (autoclaved). The stock culture was prepared as stated above and stored at $-80{ }^{\circ} \mathrm{C}$ until used.

For transformation, $50 \mu \mathrm{l}$ of chemically competent cells were thawed on ice and mixed with $\sim 100 \mathrm{ng}$ plasmid DNA. After incubation on ice for $30 \mathrm{~min}$, cells were heat shocked at $42{ }^{\circ} \mathrm{C}$ for $90 \mathrm{~s}$ and allowed to cool down before $200 \mu \mathrm{l}$ recovery media (LB media without antibiotics) were added. Cells were incubated with shaking at 37 ${ }^{\circ} \mathrm{C}$ and $1200 \mathrm{rpm}$ for $45 \mathrm{~min}$ to $1 \mathrm{hr}$. Cells were spun down at $8000 \mathrm{rpm}$ for $30 \mathrm{sec}$, $150 \mu \mathrm{l}$ supernatant media was discarded and the pellet was resuspended in the remaining supernatant to spread on to selection plates (LB-Agar plates) supplemented with respective antibiotics $(200 \mu \mathrm{g} / \mathrm{ml}$ ampicillin, $34 \mu \mathrm{g} / \mathrm{ml}$ chloramphenicol, 30 $\mu \mathrm{g} / \mathrm{ml}$ kanamycin) and incubated overnight at $37^{\circ} \mathrm{C}$. For T-Vector, 5-bromo-4-chloro3 -indolyl- $\beta$-D-galactopyranoside (X-GAL) was also supplemented to the agar plates for subsequent screening of colonies with insert. A single colony of the transformants was picked and inoculated in liquid medium for further analysis. 


\subsubsection{Plasmid and DNA-fragment purification}

For the amplification of plasmid DNA, E. coli DH5 $\alpha$ were grown for $12-16 \mathrm{~h}$ in LB medium containing the appropriate antibiotics at $37{ }^{\circ} \mathrm{C}$ at $220 \mathrm{rpm}$. Plasmids were subsequently purified via anion exchange chromatography using the Wizard Plus SV Miniprep DNA Purification System (Promega Corporation, USA), according to the manufacturer's instructions. Concentration of the isolated plasmid was determined by UV spectroscopy by measuring absorbance at $260 \mathrm{~nm}$ using the Nanodrop 2000 Spectrophotometer (Thermo Scientific, Singapore). Restriction digestion followed by agarose gel electrophoresis was performed to verify the presence of the insert and plasmid DNA. In order to purify or isolate DNA-fragments after agarose gel electrophoresis or enzymatic reactions, the Wizard SV Gel and PCR Clean-Up system was used according to the manufacturer's instructions.

\begin{tabular}{|l|c|}
\hline \multicolumn{1}{|c|}{ Reagent } & Volume \\
\hline 10x NEB Buffer & $2 \mu \mathrm{l}$ \\
\hline Restriction enzyme 1 & $0.3 \mu \mathrm{l}$ \\
\hline Restriction enzyme 2 & $0.3 \mu \mathrm{l}$ \\
\hline Plasmid/DNA & $1 \mu \mathrm{g}$ \\
\hline Autoclaved water & Variable \\
\hline Total volume & $\mathbf{2 0} \boldsymbol{\mu l}$ \\
\hline
\end{tabular}

Table 2.6: Restriction digestion reaction $\mathbf{m i x}$

\subsubsection{Polymerase chain reaction (PCR)}

Amplification of DNA fragments with point mutations and insertions was achieved by PCR. To amplify genes from plasmids and after site directed mutagenesis, a high fidelity polymerase with proof reading ability, Pfu polymerase (Thermo scientific, Singapore) was used.

\subsubsection{DNA amplification by PCR}

Plasmid/genomic DNA was used as template for PCR, set up in a total volume of 20 $\mu l$ on ice with the appropriate concentration of the constituents as mentioned below. 


\begin{tabular}{|l|c|}
\hline \multicolumn{1}{|c|}{ Reagent } & Volume \\
\hline Pfu 10X Reaction Buffer w/20 $\mathrm{mM} \mathrm{MgSO}_{4}$ & $2 \mu \mathrm{l}$ \\
\hline $10 \mu \mathrm{M}$ forward primer & $0.5 \mu \mathrm{l}$ \\
\hline $10 \mu \mathrm{M}$ reverse primer & $0.5 \mu \mathrm{l}$ \\
\hline Pfu DNA polymerase (1.2U) & $0.5 \mu \mathrm{l}$ \\
\hline Template DNA & $1-30 \mathrm{ng}$ \\
\hline 2 mM dNTP & $2 \mu \mathrm{l}$ \\
\hline Autoclaved water & Variable \\
\hline Total Volume & $\mathbf{2 0} \boldsymbol{\mu l}$ \\
\hline
\end{tabular}

Table 2.7: PCR Reaction mix

All reagents were pipetted into a PCR tube kept on ice all the time, mixing of the reagents was done by short centrifugation. PCR thermo cycler (BioMetra) was pre heated to $95{ }^{\circ} \mathrm{C}$ before reaction tubes were placed inside the machine. The following PCR thermocycler program was used:

\begin{tabular}{|c|c|c|c|}
\hline Cycle Steps & Temperature & Time & Cycles \\
\hline Lid & $95^{\circ} \mathrm{C}$ & & \\
\hline Initial denaturation & $95^{\circ} \mathrm{C}$ & $2 \mathrm{~min}$ & 1 \\
\hline Cycle denaturation & $95^{\circ} \mathrm{C}$ & $20 \mathrm{sec}$ & \multirow{3}{*}{35} \\
\hline Annealing & Variable & $30 \mathrm{sec}$ & \\
\hline Extension & $72^{\circ} \mathrm{C}$ & $1 \mathrm{~min} / \mathrm{kb}$ & \\
\hline Final extension & $72^{\circ} \mathrm{C}$ & $10 \mathrm{~min}$ & 1 \\
\hline End & $16^{\circ} \mathrm{C}$ & $\infty$ & \\
\hline
\end{tabular}

Table 2.8: Temperature cycles for DNA amplification by PCR

For cloning into pGemT vector, polyA tail amplification was done by the addition of $0.2 \mu \mathrm{l}$ of Taq polymerase and $2 \mu \mathrm{l}$ of $2 \mathrm{mM}$ dATP to the amplified DNA followed by incubation at $72{ }^{\circ} \mathrm{C}$ for $30 \mathrm{~min}$ in the PCR thermocycler to introduce a desoxyadenosine (dA) tail via ligating with the desoxythymidine overhang. dA-tailed PCR product was mixed with 6x DNA loading dye (Thermo Scientific, Singapore) prior to loading into $1 \%$ TAE-agarose gel $(40 \mathrm{ml})$ supplemented with $4 \mu \mathrm{l}$ of SYBR Safe DNA Gel Stain (Invitrogen, Singapore). Five $\mu$ l of GeneRuler 1kb DNA Ladder (Thermo Scientific, Singapore) was loaded to serve as marker. Agarose gel electrophoresis was performed in 1xTAE buffer (40 mM Tris-acetate, $\mathrm{pH} 8.5,2 \mathrm{mM}$ EDTA) at $100 \mathrm{~V}$ for $30-45 \mathrm{~min}$ and DNA fragments were then purified from the gel using the Wizard SV Gel and PCR clean-Up system (Promega, USA) according to the 
manufacturer's protocol. The product was finally eluted in 50-70 $\mu 1$ of nuclease free water.

\subsubsection{Site-directed mutagenesis}

Site-directed mutagenesis was used to introduce point mutations and insertions in existing plasmids. Site directed mutagenesis of existing plasmids was done using QuikChange protocol (Stratagene) according to manufacturer's instructions (Table 2.9 and 2.10). Forward and reverse primers were self-complimentary and contained the desired mutations (Table 2.5). After the PCR, $0.5 \mu 1$ of DpnI were added to a $10 \mu \mathrm{l}$ of the reaction mix and incubated at $37{ }^{\circ} \mathrm{C}$ for $1 \mathrm{~h}$ to digest the methylated template plasmid before transformation into E.coli $\mathrm{DH} 5 \alpha$.

\begin{tabular}{|l|c|}
\hline \multicolumn{1}{|c|}{ Reagent } & Volume \\
\hline Pfu 10X Reaction Buffer w/20 $\mathrm{mM} \mathrm{MgSO}_{4}$ & $2 \mu \mathrm{l}$ \\
\hline $10 \mu \mathrm{M}$ forward primer & $0.72 \mu \mathrm{l}$ \\
\hline $10 \mu \mathrm{M}$ reverse primer & $0.72 \mu \mathrm{l}$ \\
\hline Pfu DNA polymerase (1.2U) & $0.38 \mu \mathrm{l}$ \\
\hline Template DNA & $30 \mathrm{ng}$ \\
\hline $2 \mathrm{mM}$ dNTP & $2 \mu \mathrm{l}$ \\
\hline Autoclaved water & Variable \\
\hline Total Volume & $\mathbf{2 0} \boldsymbol{\mu l}$ \\
\hline
\end{tabular}

Table 2.9: PCR Reaction mix

\begin{tabular}{|l|l|l|l|}
\hline \multicolumn{1}{|c|}{ Cycle Steps } & \multicolumn{1}{|c|}{ Temp. $\left({ }^{\circ} \mathrm{C}\right)$} & \multicolumn{1}{|c|}{ Time } & Cycles \\
\hline Lid & $95^{\circ} \mathrm{C}$ & & \\
\hline Initial denaturation & $95^{\circ} \mathrm{C}$ & $2 \mathrm{~min}$ & 1 \\
\hline Cycle denaturation & $95^{\circ} \mathrm{C}$ & $30 \mathrm{sec}$ & \multirow{2}{*}{18} \\
\hline Annealing & Variable & $1 \mathrm{~min}$ & \\
\cline { 1 - 3 } Extension & $68^{\circ} \mathrm{C}$ & $15 \mathrm{~min}$ & \\
\hline Final extension & $68^{\circ} \mathrm{C}$ & $15 \mathrm{~min}$ & 1 \\
\hline End & $16^{\circ} \mathrm{C}$ & $\infty$ & \\
\hline
\end{tabular}

Table 2.10: Temperature cycles for DNA amplification for site-directed mutagenesis

\subsubsection{Restriction digestion and ligation}

Expression vector for cloning or vector containing gene of interest or amplified PCR product ( $1 \mu \mathrm{g}$ DNA) were digested with appropriate restriction enzymes (20-60 U) in $20 \mu \mathrm{l}$ reactions with respective buffer according to manufacturer's recommendations. 
The digestion reaction was carried out at $37^{\circ} \mathrm{C}$ for $3 \mathrm{~h}$ or overnight (Table 2.6). Digested plasmid vector DNA was incubated for $1 \mathrm{~h}$ at $37{ }^{\circ} \mathrm{C}$ with calf intestinal phosphatase (CIP) for dephosphorylation. Restriction digestion of the plasmid produces linearized plasmids (vector) and DNA fragments flanked with respective overhangs. The digests, plasmid, and inserts, were separated by $1 \%$ agarose gel electrophoresis and the respective DNA bands were extracted for purification by the Wizard Plus SV gel clean DNA Purification System. Concentrations of the purified insert and plasmid DNA were measured using a Nanodrop2000 (Thermo-scientific, Singapore).

Following purification, ligation of purified DNA fragments (insert with the vector) was carried out at room temperature (RT) for $1 \mathrm{~h}$ or overnight at $4{ }^{\circ} \mathrm{C}$. The ligation reaction was performed in a total reaction mix of $10 \mu$ containing $\sim 100 \mathrm{ng}$ of digested plasmid DNA and 500 ng DNA insert incubated with $200 \mathrm{U}$ of T4 DNA ligase (Thermo-Scientific). The reaction was conducted in T4 DNA ligase buffer. The pGem-T vector system (Promega) was used according to manufacturer's instructions, for ligation of $\mathrm{dA}$ tailed PCR products. Following the ligation, the reaction mixture was either heat shock transformed into $E$. coli DH5a cells or DNA precipitation was carried out to prepare the ligated vectors for sequential restriction enzyme digestion. Appropriate constructs were verified by purifying the plasmid DNA from the transformed colonies grown overnight at $37{ }^{\circ} \mathrm{C}$ on the selective agar plates and subjecting to restriction digestion followed by sequencing the digest using 1 st base (Singapore). The sequencing results were validated by Multalin and BioEdit programs.

\subsubsection{General cloning strategy}

The PCR product was polyadenylated at both the 5' and $3^{\prime}$ ends to allow cloning of PCR product into a vector. The polyadenylated PCR product was run on $1 \%$ agarose gel and the DNA fragments were purified from the gel using the Wizard SV Gel and PCR Clean-Up system according to the manufacturer's instructions. Ligation of the polyadenylated PCR product into pGem-T was carried out according to manufacturer's instruction. The ligation mixture was transformed into DH5 $\alpha$ and plated on an ampicillin LB plate (200 $\mu \mathrm{g} / \mathrm{ml}$ ampicillin) supplemented with $25 \mu 1$ of $40 \mathrm{mg} / \mathrm{ml} \quad \mathrm{X}$-gal (5-bromo-4-chloro-3-indolyl- $\beta$-D-galactopyranoside) in 
$\mathrm{N}, \mathrm{N}$-dimethylformamide (DMF). X-gal enables blue white screening and only white colonies containing insert were inoculated into $6 \mathrm{ml} \mathrm{LB}$ medium supplemented with ampicillin. This culture was grown overnight in a shaking incubator at $37{ }^{\circ} \mathrm{C}, 220$ rpm. The plasmids were purified using Wizard Plus SV Miniprep DNA Purification System (Promega Corporation, USA) and their concentrations were measured using a Nanodrop 2000 Spectrophotometer (Thermo-scientific, Singapore) at $260 \mathrm{~nm}$. The plasmids were sequenced by 1 st base (Singapore). pGem-T vector with inserted sequences was digested with respective restriction enzymes either simultaneously or sequentially and the purified inserts were ligated into the pHue vector digested with the same enzymes and dephosphorylated by CIP.

In this study, the expression vector pHue was used for the heterologous expression of protein constructs in E. coli. Proteins were expressed with a N-terminal fusion to hexa-histidine tagged ubiquitin $\left(\mathrm{His}_{6} \mathrm{Ub}\right)$, which improves solubility and enables easy protein purification by immobilized metal-ion affinity chromatography (IMAC) [180]. The resultant pHue vector was transformed in DH5 $\alpha$ and screened for ampicillin resistant colonies, which was eventually picked and grown in LB medium supplemented with ampicillin and the correct insert was confirmed by sequencing. Mutations in the inserts were generated by site directed mutagenesis. Cleavage of the tag by the deubiquitinating enzyme Usp2 leaves the native N-terminus of the protein of interest [181].

The coding sequence of the mature $\alpha$ (long) and the $\beta$ (short) isoform of Oryza sativa Rubisco activase (Uniprot accession code: P93431-1 and P93431-2, expressed amino acids: 48-466 and 48-433 respectively), Thioredoxin-f of Oryza sativa (Uniprot accession code: Q8S091, expressed amino acids 73-186) and the $\alpha$ (long) and the $\beta$ (short) isoform of Rubisco activase of Agave tequilana (locus 3705 and 27298, expressed amino acids: 57-474 and 58-435 respectively [182]) were synthesised (GenScript, USA) with flanking SacII and HindIII restriction sites at 5' and 3' sites respectively and were cloned in pUC57 plasmid. pUC57 plasmid bearing subsequent insert was transformed into DH5 $\alpha$ cells and plated on ampicillin LB-agar plate (200 $\mu \mathrm{g} / \mathrm{ml})$. The successful colonies were used for the amplification of plasmid DNA in $E$. coli DH5 $\alpha$ which were grown for 12-16 h in LB medium containing ampicillin (200 $\mu \mathrm{g} / \mathrm{ml}$ ) at $37^{\circ} \mathrm{C}$ with $220 \mathrm{rpm}$ shaking. The plasmids were isolated as described above 
using Wizard Plus SV Miniprep DNA Purification System (Promega Corporation, USA) by following manufacturer's protocol. Both pUC57 and pHue plasmids were double or sequentially digested with SacII and HindIII restriction enzymes. The digested plasmid and plasmid insert was loaded on to $1 \%$ agarose gel and purified as described above. Ligation of these insert fragment with linearized plasmids was performed at room temperature for $1 \mathrm{~h}$ with a $10 \mu \mathrm{l}$ reaction mixture containing $1 \mu \mathrm{l}$ T4 DNA ligase buffer, $2 \mu \mathrm{l}$ vector, $6 \mu \mathrm{l}$ insert fragments and $1 \mu 1 \mathrm{~T} 4$ DNA ligase (Thermo-Scientific). The reaction mixture was transformed into E.coli DH5a cells and subsequently the plasmid was purified as described above. Approximately $500 \mathrm{ng}$ of plasmid was test cut using SacII and HindIII, to check the presence of genes of interest. These plasmids were transformed into E.coli BL21 competent cells for protein purification.

The coding sequence of the N-terminally truncated Rubisco activase (residue 67-386) from Oryza sativa was amplified with the primer pairs $\Delta$ N67 OsRcaSacIIfor and T7term from the plasmid pHueOsRca $\beta$. The PCR product was subsequently dA tailed, cloned into vector pGemT and then double digested with SacII and HindIII and finally ligated into the vector pHue. The expression plasmid was named as pHue $\Delta$ N67OsRca $\beta$. Mutations in the inserts were generated by site-directed mutagenesis unless indicated otherwise with the appropriate primer pairs (Table 2.5). All the mutations were confirmed by sequencing ( $1^{\text {st }}$ base, Singapore).

The WT template plasmids from rice and agave were used to introduced point mutations and insertions by PCR mutagenesis following the Quikchange protocol (Stratagene) for generating chimeric mutants of Rca with swapped domains and regions (pHueRcaßAtNOsC, pHueRcaßOsNAtC, pHueAtRcaaOsTail, pHueOsRca $\alpha$ AtTail) as shown in Fig. 2.1. Primers were designed for the removal of ApoI site from pUC57 backbone of AtRca $\beta$ and for the insertion of ApoI restriction enzyme site into OsRca $\beta$ via a substitution mutation without changing the amino acid composition. Similarly, primers were designed for the insertion of serine into AtRca $\alpha$ and OsRca $\alpha$ after amino acid residue G381 to create a BamH1 restriction enzyme site (Table 2.5) to facilitate the formation of chimeric mutant. Chimeric constructs between rice and agave were also cloned and expressed as His6-Ubiquitin fusions for protein purification. 


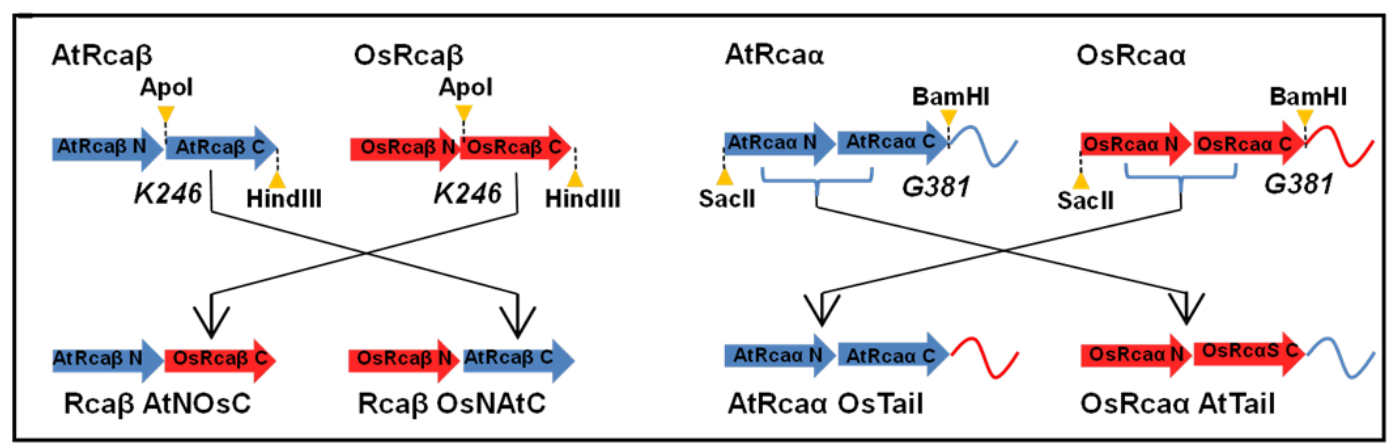

Fig. 2.1: Schematic representation of the generation of chimeric mutants. N-terminal domain corresponding to the regions after the lysine 246 in short activase isoform was interchanged to generate two chimeric mutants. Similarly, C-tail extension region after glycine 381 was switched for long activase isoform of rice and agave to generate another two chimeric mutants.

\subsection{Protein biochemistry}

\subsubsection{Protein analytical methods}

Protein sequence alignment- Protein sequences were aligned using the programs MultAlign [183] or Espript [184].

Protein structure analysis - Pymol [185].

Protein quantification- Protein mixtures were quantified by Bradford (BioRad) assay using bovine serum albumin as a standard. Two to ten $\mu \mathrm{g}$ of bovine serum albumin (BSA) was added to a mixture of $500 \mu \mathrm{l}$ autoclaved $\mathrm{H}_{2} \mathrm{O}$ and $500 \mu 1$ Bradford reagent and absorbance of the samples were measured at $595 \mathrm{~nm}$ on a spectrophotometer. Pure proteins were quantified by their absorbance at $280 \mathrm{~nm}$ by Nanodrop 2000 (Thermoscientific, Singapore) and the concentration was calculated using the extinction coefficients listed in Table 2.11. Extinction coefficients were calculated with the program ProtParam [186]. Extinction coefficients of the point mutations not listed were the same as for the respective wild-type OsRca. Final concentration of the protein is calculated using extinction coefficient and molecular weight.

$$
C=\left(A_{280} / \varepsilon\right) \times \text { Molecular Weight }
$$

C- Concentration of the protein in $\mathrm{mg} / \mathrm{ml}$

$\mathrm{A}_{280}-$ Absorbance measured by Nanodrop at $280 \mathrm{~nm}$ 
$\varepsilon-$ Extinction coefficient $\mathrm{M}^{-1} \mathrm{~cm}^{-1}$

\begin{tabular}{|c|c|c|}
\hline Protein & Extinction coefficient $\left(M^{-1} \mathrm{~cm}^{-1}\right)$ & Molecular weight (Da) \\
\hline OsRca $\alpha$ & 45840 & 46485.5 \\
\hline OsRca $\beta$ & 44350 & 42961.6 \\
\hline OsTrx-f & 12490 & 12569.7 \\
\hline$\Delta \mathrm{N} 67$ OsRca $\beta$ & 32890 & 35563.7 \\
\hline AtRca $\alpha$ & 45840 & 46491.7 \\
\hline AtRca $\beta$ & 41370 & 42041.9 \\
\hline OsRbc (active site) & 103250 & 67612.1 \\
\hline OsRca $\beta \mathrm{W} 15 \mathrm{~A}$ & 38850 & 42846.5 \\
\hline OsRcaßY214A & 42860 & 42869.5 \\
\hline RcaßAtNOsC & 42860 & 42158.8 \\
\hline RcaßOsNAtC & 42860 & 42358.2 \\
\hline OsRca $\alpha$ AtTail & 45840 & 46498.5 \\
\hline AtRca $\alpha$ OsTail & 45840 & 46652.8 \\
\hline$\Delta \mathrm{N} 21$ OsRca2 & 48360 & 42416.3 \\
\hline NtRca & 39880 & 42759 \\
\hline
\end{tabular}

Table 2.11: Extinction co-efficient and molecular weight

\subsubsection{SDS-PAGE and Native PAGE}

Protein analysis was performed under reducing, denaturing conditions by sodiumdodecylsulfate polyacrylamide gel electrophoresis (SDS-PAGE) [187]. In this method, the proteins were resolved based on their molecular weight and electrophoretic mobility. Gels were prepared as per standard protocol [179]. Samples were mixed with $5 \mathrm{X}$ SDS-sample buffer, boiled for $5 \mathrm{~min}$ at $100{ }^{\circ} \mathrm{C}$, and centrifuged for $2 \mathrm{~min}$ at RT at $16000 \mathrm{xg}$. Centrifuged samples were loaded into their respective wells and gel electrophoresis was performed in Mini Protean (Bio-rad) electrophoresis 
chambers in SDS-electrophoresis migration buffer (with SDS) at constant voltage of $100 \mathrm{~V}$ for $20 \mathrm{~min}$ followed by $140-180 \mathrm{~V}$ for the rest of the run. The proteins were electrophoretically separated in denatured conditions.

Native-PAGE samples were mixed with $5 \mathrm{x}$ native loading buffer (without SDS and $\beta$ mercaptoethanol). Samples were not boiled and the gel and tank buffer solutions were without SDS. Samples were loaded into their respective gel and subsequently into Bio-Rad electrophoresis chambers in the presence of their respective running buffer (Table 2.4). Gel electrophoresis was carried out in Mini Protean (Bio-Rad) electrophoresis chambers in the presence of electrophoresis migration buffer employing a starting constant voltage of $100 \mathrm{~V}$ followed by $140-160 \mathrm{~V}$ for the rest of the run. The proteins were electrophoretically separated in native conditions at $4{ }^{\circ} \mathrm{C}$ and at RT in denatured conditions.

\subsubsection{Coomassie blue staining of SDS and native PAGE}

Proteins that were separated by electrophoresis were briefly washed in water before staining by incubation of the gel in Coomassie Brilliant Blue (40\% methanol, $10 \%$ acetic acid, $0.1 \%$ Coomassie Brilliant Blue R-250) Coomassie blue is a triphenylmethane coloring agent. This dye integrates with cationic, nonpolar, and hydrophobic side chains of a polypeptide. Hence, Coomassie blue staining was used for the visualization of protein bands in polyacrylamide gels. Protein bands were fixed and stained by incubating the gels for $1 \mathrm{~h}$ with Coomassie blue staining solution. Consequently, the gel was incubated in destaining solution for $1 \mathrm{~h}$ or more to remove the background staining until the bands were clearly visible.

\subsubsection{Western blotting and immunodetection}

After SDS-PAGE, proteins were transferred onto a nitrocellulose membrane using transfer buffer (50 mM Tris-HCl pH 8.0, 20\% methanol, $192 \mathrm{mM}$ glycine) in a semidry western blot apparatus (BioRad). This transfer was performed at 15 volts for 15 min or 10 volts for $30 \mathrm{~min}$. The membrane was stained with Ponceau S staining solution $(0.1 \%(\mathrm{w} / \mathrm{v})$ Ponceau S, 5\% acetic acid) to ensure efficient transfer and destained with Milli-Q water before proceeding with the immunodetection. The 
membrane was blocked with 5\% (w/v) skimmed milk powder in TBS (50 mM Tris$\mathrm{HCl} \mathrm{pH} 8.0,137 \mathrm{mM} \mathrm{NaCl}, 2.7 \mathrm{mM} \mathrm{KCl}$ ) for 1 hour at room temperature.

The primary antibody anti-Rca or anti-Rubisco (Rabbit, polyclonal,) (Agrisera, Sweden) was diluted to 1:5000 in TBS-T (TBS, 0.1\% (v/v) TWEEN 2.0) and incubated with the membrane for $1 \mathrm{~h}$ at RT. The membrane was washed thrice with TBS-T and subsequently incubated in TBS-T for $15 \mathrm{~min}$. The secondary antibody peroxidase-conjugated anti-rabbit was diluted to 1:10000 in TBS-T and incubated with the membrane for $1 \mathrm{~h}$ at RT. The membrane was washed again (as above). The HRP coupled antibody was detected by enhanced chemiluminescence (ECL) staining with a 1:1 mix of ECL detection reagent 1 and 2 (Thermo Scientific) on an LAS-3000 Imagining system (Fuji).

\subsubsection{Circular dichroism (CD) -spectroscopy}

Structural integrity of purified Rca at different temperature was analyzed by CDspectroscopy. Proteins were desalted into buffer $\left(20 \mathrm{mM} \mathrm{K} \mathrm{HPO}_{4} / \mathrm{KH}_{2} \mathrm{PO}_{4} \mathrm{pH} 8.0\right.$ ) using the Bio-Spin6 chromatography column (Bio-Rad) according to manufacturer's instructions. Protein concentration was adjusted to a final concentration of $1 \mathrm{mg} / \mathrm{ml}$ and the CD spectra were recorded on a Chirascan ${ }^{\mathrm{TM}} \mathrm{CD}$-spectrometer (average of 3 passes, $240 \mathrm{~nm}$ to $190 \mathrm{~nm}, 0.1 \mathrm{~nm}$ data pitch, $20-50{ }^{\circ} \mathrm{C}$ ).

\subsubsection{Test expression of proteins}

Glycerol stock of the BL21 cells carrying the activase and Rubisco constructs were inoculated in LB media supplemented with respective antibody and grown overnight at $37{ }^{\circ} \mathrm{C}$ with shaking $(220 \mathrm{rpm})$ in an Innova44 incubator (New Brunswick Scientific). This overnight culture $(50 \mu \mathrm{l})$ was subcultured into a couple of fresh $5 \mathrm{ml}$ LB media supplemented with the same antibody. This culture was grown at $37{ }^{\circ} \mathrm{C}$ with shaking until the optical density reached $\sim 0.6$ at a wavelength of $600 \mathrm{~nm}$ $\left(\mathrm{OD}_{600}\right)$. Two different temperatures $\left(37^{\circ} \mathrm{C}\right.$ and $\left.23^{\circ} \mathrm{C}\right)$ were tested for expression of proteins. One culture was moved to $23{ }^{\circ} \mathrm{C}$ with shaking, while the other was kept at $37{ }^{\circ} \mathrm{C}$. Both cultures were induced with $0.5 \mathrm{mM}$ isopropyl-D-thiogalactopyranoside (IPTG). For co-expression of construct GroELES, initially $0.2 \%$ L-arabinose was added and $30 \mathrm{~min}$ later $0.5 \mathrm{mM}$ of IPTG was added to express the protein of interest 
in the construct. Cells were harvested by centrifugation at $4000 \mathrm{xg}$ for $10 \mathrm{~min}$ after 4 $\mathrm{h}$ or $12 \mathrm{~h}$ of induction. After removing the supernatant media, the cells were resuspended in $1 \mathrm{ml}$ of lysis buffer $(50 \mathrm{mM}$ Tris- $\mathrm{HCl} \mathrm{pH} \mathrm{8.0,50} \mathrm{mM} \mathrm{NaCl})$. Cells were lysed on ice by sonication at 30\% amplitude for $50 \mathrm{~s}$ using $10 \mathrm{~s}$ pulses alternated with $50 \mathrm{~s}$ of pause. The lysate $(50 \mu \mathrm{l})$ was added with $12.5 \mu \mathrm{l}$ X SDS sample buffer for analysis of total protein expression (total lysate sample). The lysate (300 $\mu \mathrm{l})$ was clarified by centrifugation at $6000 \mathrm{xg}$ and $4{ }^{\circ} \mathrm{C}$ for $15 \mathrm{~min}$ and $50 \mu \mathrm{l}$ of the supernatant was added with $12.5 \mu \mathrm{L}$ X SDS sample buffer for analysis of soluble protein expression (soluble sample). After discarding the remaining supernatant, the pellet was washed with $300 \mu \mathrm{l}$ MilliQ water and centrifuged at $16000 \mathrm{xg}$ and $4{ }^{\circ} \mathrm{C}$ for 15 min. The pellet was redissolved in $72.5 \mu \mathrm{l} 2 \mathrm{X}$ SDS sample buffer for analysis of insoluble protein expression (insoluble sample). The collected samples were heat denatured using a heat block at $100{ }^{\circ} \mathrm{C}$ and centrifuged at $16000 \mathrm{xg}$ and RT for $3 \mathrm{~min}$ prior to loading on $12.5 \%$ SDS polyacrylamide gel. Five $\mu 1$ of pre-stained marker (PageRuler Plus Prestained Protein Ladder 10-250 kDa; Thermo Scientific) was loaded alongside the samples. SDS-PAGE gel electrophoresis was performed at a starting voltage of $120 \mathrm{~V}$ followed by $160 \mathrm{~V}$ for the rest of the run.

\subsubsection{Expression and purification}

All the activase proteins and its variants used in this study were expressed in BL21 as a $\mathrm{His}_{6} \mathrm{Ub}$-fusion constructs using pHue expression vectors. BL21 cells carrying the respective plasmid construct were grown in LB supplemented with respective antibodies at $37{ }^{\circ} \mathrm{C}$ in an Innova44R incubator (New Brunswick Scientific) at moderate shaking $(200 \mathrm{rpm})$ to an optical density at a wavelength of $600 \mathrm{~nm}$ $\mathrm{OD}_{600}=0.6$. The culture was then cooled down at $23{ }^{\circ} \mathrm{C}$ for $\sim 20 \mathrm{~min}$ and induced with $0.5 \mathrm{mM}$ IPTG for $\sim 16 \mathrm{~h}$ at $23{ }^{\circ} \mathrm{C}$. Following induction, the culture was allowed to grow for $\sim 16 \mathrm{~h}$ at $200 \mathrm{rpm}$ at $23{ }^{\circ} \mathrm{C}$. After incubation, E.coli cells were harvested by centrifugation in JA-10 centrifuge with JA 10 rotor (Beckman Coulter) at $4000 \mathrm{xg}$ for 20 min at $4{ }^{\circ} \mathrm{C}$. Cell pellets were either stored at $-20{ }^{\circ} \mathrm{C}$ or directly lysed as described. 


\subsubsection{Purification of $\mathrm{His}_{6} \mathrm{Ub}$-fusion constructs}

$\mathrm{His}_{6} \mathrm{Ub}$-fusion constructs were captured by immobilized metal affinity chromatography (IMAC) and after cleavage of the ubiquitin moiety, further purified by anion exchange and gel filtration chromatography. Harvested cells from 11 of bacterial culture were resuspended in $40 \mathrm{ml}$ lysis buffer A $(50 \mathrm{mM}$ Tris- $\mathrm{HCl} \mathrm{pH} 8.0$, $50 \mathrm{mM} \mathrm{NaCl}, 10 \mathrm{mM}$ imidazole) supplemented with $0.3 \mathrm{mg} / \mathrm{ml}$ lysozyme to aid lysis and with $1 \mathrm{mM}$ PMSF for protease inhibition just before sonication. The cells were disrupted by ultrasonication on ice for $5 \mathrm{~min}$ with a sonicator $(15 \mathrm{~s}$ bursts with $30 \mathrm{~s}$ cooling on ice between bursts). Following removal of cell debris by centrifugation in an Avanti J-25 with JA25.50 rotor (Beckman Coulter) for 40 min at $4{ }^{\circ} \mathrm{C}$ and 20000 $\mathrm{g}$, the supernatant was applied to an affinity column packed with Ni-NTA resin (Thermo Scientific) equilibrated with lysis buffer A (Table 2.4). The His ${ }_{6} \mathrm{Ub}$-fusion protein was eluted using elution buffer $(50 \mathrm{mM}$ Tris- $\mathrm{HCl} \mathrm{pH} 8.0,50 \mathrm{mM} \mathrm{NaCl}$, and $200 \mathrm{mM}$ imidazole). The ubiquitin moiety was cleaved overnight at $4{ }^{\circ} \mathrm{C}$ using the deubiquitylating enzyme Usp2. The protein solution was then applied to an anion protein exchanger MonoQ 10/100 column (GE); proteins were eluted with a linear salt gradient of $500 \mathrm{mM} \mathrm{NaCl}$. Fractions containing protein of interest were combined and concentrated. Later, to further purify the proteins, size exclusion chromatography was performed by loading sample onto Superdex 200 16/600 column. Proteins were eluted with purification buffer A (Table 2.4). Fractions containing the protein were pooled and concentrated in an Amicon Ultra-15 centrifugal filter units (50-100 kDa cut-off) (Millipore, USA), 5\% glycerol was added and the protein was stored in aliquots at -80 ${ }^{\circ} \mathrm{C}$ after flash-freezing in liquid $\mathrm{N}_{2}$ for future use.

\subsubsection{Purification of Rubisco from the leaves of Oryza sativa}

Leaves of Oryza sativa were a kind gift from Prof. Prakash Kumar (NUS, Singapore). The Oryza sativa Rubisco $\left(\mathrm{OsRb}_{8} \mathrm{~S}_{8}\right)$ was purified from the leaves as previously described [188], with slight modifications. Briefly, $8 \mathrm{~g}$ of leaves from Oryza sativa were homogenized in liquid $\mathrm{N}_{2}$ using mortar and pestle to make fine powder and extracted with $40 \mathrm{ml}$ of Rubisco extraction buffer $(50 \mathrm{mM}$ Tris-Hcl pH 8.0, $50 \mathrm{mM}$ Nacl, $1 \mathrm{mM} \mathrm{Na} 2$ EDTA, 12.5\% Glycerol) containinng $1 \mathrm{mM}$ PMSF, one tablet of protease inhibitor cocktail, $5 \mathrm{mM}$ DTT and $2 \%$ Polyvinypyrrolidone (PVPP). The 
extract was filtered through four layer of miracloth. The cells were further disrupted by ultrasonication on ice for $5 \mathrm{~min}$ with a sonicator (15 s bursts with $30 \mathrm{~s}$ cooling on ice between bursts). Cell debris was removed by centrifugation in an Avanti J-25 with JA25.50 rotor (Beckman Coulter) for $30 \mathrm{~min}$ at $4{ }^{\circ} \mathrm{C}$ at $39000 \mathrm{xg}$. The supernatant was subjected to $33 \%$ ammonium sulphate $\left((\mathrm{NH} 4)_{2} \mathrm{SO} 4\right)$ precipitation and centrifuged for $30 \mathrm{~min}$ at $4{ }^{\circ} \mathrm{C}$ at $39000 \mathrm{xg}$. The supernatant was further subjected to $55 \%$ ammonium sulphate precipitation and centrifuged as earlier. The pellet obtained was dissolved in Rubisco resuspension buffer (Table 2.4). The resuspended solution was dialyzed extensively against Rubisco dialysis buffer overnight and applied to an anion protein exchanger MonoQ 10/100 (GE) column equilibrated with Rubisco purification buffer A. Protein was eluted with a linear salt gradient of up to $500 \mathrm{mM} \mathrm{NaCl}$ using Rubisco purification buffer B. Fractions containing Rubisco were combined and concentrated. To further purify the protein, size exclusion chromatography was performed by loading the protein onto a Superdex 200 16/600 column. Protein was eluted with Rubisco purification buffer A (Table 2.4). Fractions containing the protein were pooled and concentrated using Amicon Ultra-15 Centrifugal Filter Units (Millipore, USA). Protein was stored in aliquots at $-80{ }^{\circ} \mathrm{C}$ after flash-freezing in liquid $\mathrm{N}_{2}$.

\subsubsection{Analytical size exclusion chromatograpghy}

Analytical size exclusion chromatograpghy of proteins was performed on a Superdex 200 PC3.2/30 increase column equiliberated with buffer A or buffer A supplemented with $5 \mathrm{mM} \mathrm{MgCl}_{2}$ and $1 \mathrm{mM}$ ATP using Akta micro system (GE Healthcare). Protein-containing samples (50 $\mu \mathrm{l}$ each) were injected and runs were performed at RT at a flow rate of $0.075 \mathrm{ml} / \mathrm{min}$ in respective buffer. UV signal for the proteins were detected at $280 \mathrm{~nm}$. The positive fractions were collected and analyzed with SDS or native PAGE gels. The column was calibrated with globular proteins of known molecular weights like blue dextran $2000 \mathrm{kDa}$, ovalbumin $44 \mathrm{kDa}$, conalbumin 75 $\mathrm{kDa}$, aldolase $158 \mathrm{kDa}$, ferritin $440 \mathrm{kDa}$, thyroglobulin $669 \mathrm{kDa}$ from HMW gel filtration calibration kit (GE Healthcare). 


\subsubsection{Enzymatic assay}

\subsubsection{ATPase assay}

ATPase activity of the activases was measured spectrophotometrically using a coupled assay monitoring the oxidation of reduced nicotinamide adenine dinucleotide (NADH) [189] in buffer (100 mM Tricine $\mathrm{pH} 8.0,5 \mathrm{mM} \mathrm{MgCl}_{2}, 2 \mathrm{mM}$ phosphoenolpyruvate, $0.5 \mathrm{mM} \mathrm{NADH}, 2 \mathrm{mM}$ ATP, $20 \mathrm{U} / \mathrm{ml}$ pyruvate kinase, 30 $\mathrm{U} / \mathrm{ml}$ lactate dehydrogenase) with a final Rca concentration of $5 \mu \mathrm{M}$ at $25{ }^{\circ} \mathrm{C}$ unless otherwise stated. The oxidation of $\mathrm{NADH}$, which is directly coupled to the generation of ADP, was followed by the reduction in the absorbance at $340 \mathrm{~nm}$ on a UV-1800 spectrophotometer (Shimadzu). The final volume of the reaction was $100 \mu 1$.

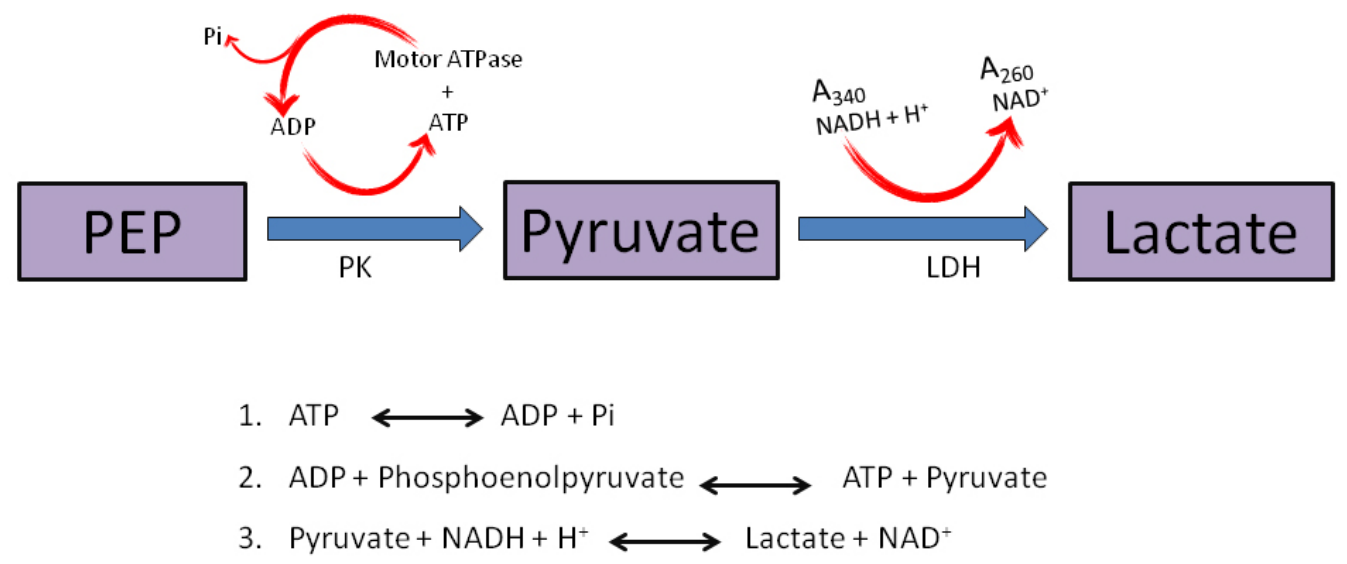

Fig. 2.2: Representation of the reactions that occurred during an ATPase activity assay. A decrease in absorbance indicates oxidation of reduced Nicotinamide adenine dinucleotide (NADH), which is proportional to ATP hydrolysis rates.

\subsubsection{Rubisco assay}

Rubisco activity assay was performed as previously described [190] with minor modifications. This assay measures the oxidation of NADH specrophotometrically in a $100 \mu \mathrm{l}$ reaction volume using a UV-1800 spectrophotometer (Shimadzu) at $340 \mathrm{~nm}$. The assay was performed in a buffer $(100 \mathrm{mM}$ Tricine $\mathrm{pH} 8.0,5 \mathrm{mM} \mathrm{MgCl} 2)$ with 3 $\mu l$ coupling enzymes mixture (creatine P-kinase $(2.5 \mathrm{U} / \mathrm{ml}$ ), glyceraldehyde-3-P dehydrogenase (2.5 units/ml), 3-phosphoglycerate kinase and triose-P isomerase/glycerol-3-P dehydrogenase (20/2 units/ml)), $20 \mathrm{mM} \mathrm{NaHCO} 3,0.5 \mathrm{mM}$ $\mathrm{NADH}, 2 \mathrm{mM}$ ATP, $10 \mathrm{mM}$ creatine-P, $1 \mathrm{mM}$ RuBP, with a final concentration of 0.5 
$\mu \mathrm{M}$ Rubisco active sites and 5 Rca protomer unless otherwise stated. The oxidation of $\mathrm{NADH}$, which is directly coupled to the generation of 3PG, was followed by the reduction in the absorbance at $340 \mathrm{~nm}$ on a spectrophotometer.

Fully carbamylated Rubisco (ECM) complex was made by incubating $20 \mu \mathrm{M}$ Rubisco active sites with $20 \mathrm{mM} \mathrm{NaHCO} 3,10 \mathrm{mM} \mathrm{MgCl} 2$ in buffer A (Table 2.4). RuBP inhibited Rubisco (ER) complex was made in same buffer by incubating $20 \mu \mathrm{M}$ Rubisco active sites with $4 \mathrm{mM}$ EDTA for $10 \mathrm{~min}$ at $25^{\circ} \mathrm{C}$. Later to this, $1 \mathrm{mM} \mathrm{RuBP}$ is added to make the final ER complex. RuBP was synthesised enzymatically from ribose-5-phosphate [191] and purified using anion exchange chromatography [192].

Activity of Rubiscos (ECM, ER) was calculated by the 3PG generated and activation rate of inhibited Rubiscos (ER to ECM) complexes is measured by 3PG produced with the addition of the respective activases. The $3 \mathrm{PG}$ produced was plotted against time to determine the activity of Rubisco in the presence and absence of activase. The rate of 3PG produced is directly proportional to the activity of the Rubisco. For quantitative analysis of Rca and its variants, the rate of 3PG produced or the rate ECM formation from ER was measured for the initial 4 min and compared with its wild type activase relative to the background rate of Rubisco activation.

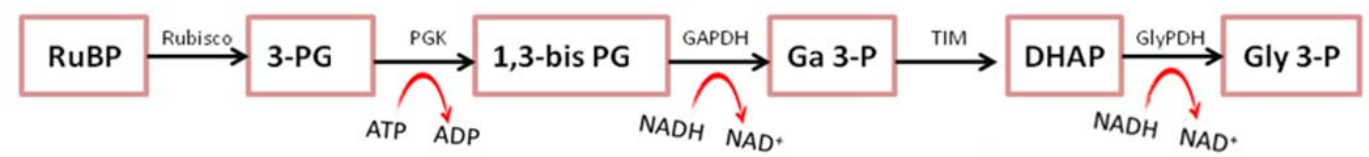

Fig. 2.3: Representation of reactions that occurred during a Rubisco activity assay. For each carboxylation of RuBP, two molecules of 3-phosphoglycerate (3-PG) were produced by Rubisco which were phosphorylated by 3-PG kinase (PGK). Glyceraldehyde-3-P dehydrogenase (GAPDH) reduces the bis-PGA to an aldehyde (Ga-3-P). Triose-phosphate isomerase (TIM) produces di-hydroxyacetone (DHAP) from Ga-3-P, glycerol-p dehydregenase (GlyPDH) reduces DHAP to glycerol 3-phosphate (Gly 3-P). Four NADH being oxidized per RuBP, leading to a decrease in absorbance at 340nm.

\subsubsection{Variations of enzymatic assay}

To investigate the effects of Trx-f and DTT, $25 \mu \mathrm{M} \alpha$ isoform was incubated at room temperature with $3.6 \mu \mathrm{M}$ rice Trx-f and/or $5 \mathrm{mM}$ DTT for $10 \mathrm{~min}$ before conducting assays. 
Thermostability test was done by heating the Rca at indicated temperature for $10 \mathrm{~min}$ then the assays were conducted at $25^{\circ} \mathrm{C}$ as described above.

To investigate the effects of temperature on the ATPase activity and Rubisco activation by activases, reaction mixes were incubated at the respective temperatures on a heat block for $10 \mathrm{~min}$ prior to measurement. The chamber of UV-1800 spectrophotometer (Shimadzu, Japan) was also heated using its thermostat along with quartz cuvette to the respective temperatures prior to measurement for $10 \mathrm{~min}$. The assay was initiated by mixing the activase to reaction mix after 10 min of heating and measurement was done at respective temperatures.

Subunit doping assays were performed by mixing mutant Rca in varying ratio with WT Rca for 10 min to yield a final concentration of $5 \mu \mathrm{M}$ total Rca in reaction mix. The Rubisco reactivation assay was then performed as described in previous section. To demonstrate the poisoning effect of subunit doping, WT Rca concentration was kept constant and mutant concentration was varied in different proportion and then the ATPase assay was performed as routine. To observe and analyse the inhibitory or stimulatory effect of mutant Rca on WT Rca, data was fitted to the theoretical plots for the population of hexamers at various ratios of WT to mutant assuming random mixing generated by mathematical modelling as earlier reported for other AAA+ proteins $[193,194]$. In this approach, mutant subunits are mixed with WT subunits to generate heterohexamer ensembles. Incorporation of mutant subunits into hexamers occurs according to a binomial distribution dictated by the WT: mutant ratio as:

$$
P(x)=\left(\begin{array}{c}
\mathrm{n} \\
\mathrm{x}
\end{array}\right) p^{\mathrm{x}}(1-p)^{\mathrm{n}-\mathrm{x}}
$$

Where $P$ is the probability that a hexamer (therefore, $\mathrm{n}=6$ ) contains $\mathrm{x}$ mutant subunits and $\mathrm{p}$ is the probability that a mutant subunit is incorporated. Experiments demonstrated that mutant and WT subunits have similar probability of being incorporated into a hexamer [193-195]. Consequently, $p$ is calculated as the molar ratio of mutant and WT protein present:

$$
p=\frac{\text { Mutant }}{(\text { Mutant }+ \text { WT })}
$$


Therefore, for any specified percentage of mutant subunits the probability distribution of Rca hexamers containing $0,1,2,3,4,5$ or 6 mutant subunits can be derived (see section 5). Activity versus $p$ plots could then be generated assuming each WT subunit makes an equal contribution to the total activity ( $1 / 6^{\text {th }}$ per subunit). Consequently, if subunits within the hexamer operate independently then activity should decline in a linear manner upon incorporation of defective mutant subunits. Conversely, if subunits are coupled then a specific number of subunits will be sufficient to eliminate activity. Thus zero activity is assigned to hexamers that are in breach of a specific threshold number of mutant subunits. In this way activity versus $p$ plots could be generated assuming one or more, two or more, three or more, four or more, five or more mutant subunits are required to eliminate activity.

The basic assumptions applied for developing mathematical models can be summarised as follows [194]:

1. The functional unit is hexamer

2. All subunits have similar tendencies to form oligomers.

3. Incorporation of one malfunctioning subunit into a hexamer reduces the activity by one-sixth of the wild type activity.

4. Within the observation window, activity is proportional to the number of active hexamers; thus bigger the fraction of the active units, the more activity is observed.

5. If inter-subunit coupling occurs, a certain number of incorporated mutant subunits into the hexamer causes complete loss of activity.

Assumptions 1-4 account for a linear decrease of activity upon incorporation of subunits as would be expected if subunit activity was independent of its neighbour, whereas assumption 5 includes a basis for inter-subunit coupling that gives rise to steep, non-linear decrease of activity. 


\section{Biochemical characterization of the Rubisco activation system of the staple crop Oryza sativa (rice)}


In plants, Rubisco activase (Rca) is an essential molecular chaperone due to its necessary role in maintaining Rubisco activity in vivo [110]. Rca is highly sensitive to heat stress which limits its capability to sustain the activation state of Rubisco at moderately high temperature $[10,11]$. Studies performed on arabidopsis revealed that improving the thermostability of activase can increase growth and photosynthetic efficiency under moderate heat stress $[12,13]$. Therefore, Rca is considered as a promising target for crop improvement, especially considering predicted climatic changes in future [18]. However, almost all detailed biochemical data concerning Rubisco activase function is derived from organisms such as arabidopsis, tobacco and spinach. This work focussed on biochemical characterization of the Rubisco activation system of an economically and socially important global crop species, Oryza sativa (rice) which could be genetically modified for facile application and commercialisation derived from such biochemical work.

Rice possesses two isoforms of Rca as a result of alternate splicing of a single gene transcript into a long $(\alpha)$ isoform $(46 \mathrm{kDa})$ and a short $(\beta)$ isoform $(43 \mathrm{kDa})$. The two isoforms are identical except for the $\alpha$ isoform having an additional 33 amino acids at the C-terminus, also substituting five amino acid immediately preceding this extension [144]. This C-terminal extension consists of a pair of cysteine residues responsible for regulating Rca activity via changes in the redox state of chloroplast, which in arabidopsis is modulated by thioredoxin-f [146]. The distribution and properties of Rca varies significantly among and within species. In most plants, the two isoforms are present in equimolar quantities, however, in rice, the $\beta$ isoform is much more abundant than the $\alpha$ isoform $[112,144]$ under non-stressed conditions. Importantly, heat stress in rice leads to dramatic upregulation of Rca [196]. Also, it was reported to be the most upregulated protein under heat stress in the wild rice species Oryza meridionalis [197], thus showing a relative changes in protein abundance and gene expression of the two Rca isoforms with heat. A biochemical characterization of Rca isoforms in rice has recently been reported [177]. In this chapter, we present a complementary detailed characterization of the two Rca isoforms covering various aspects of activase function such ATPase and Rubisco activase activity, redox regulation, thermostability and subunit-subunit interaction to interrogate this Rubisco activation system from the model plant Oryza sativa. 


\subsection{Protein Purification}

\subsubsection{Purification of Oryza sativa Rubisco activase (Rca) isoforms}

Long and short isoforms of Rubisco activase from Oryza sativa (OsRca $\alpha$ and OsRca $\beta$ respectively) were cloned and purified as described in materials and methods. All Rca constructs and mutants used in the study were heterologously expressed in E.coli BL21 as a N-terminal fusion with a hexa-histidine tagged ubiquitin ( $\mathrm{His}_{6} \mathrm{Ub}$ ) to improve the solubility of the respective protein upon expression $[180,181]$.

A

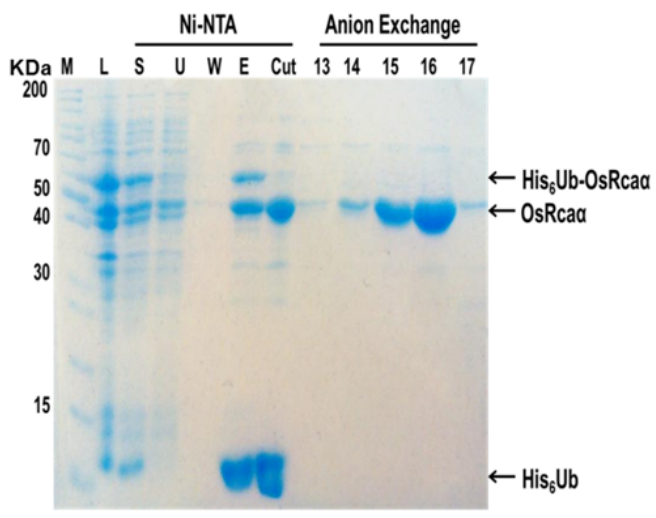

C

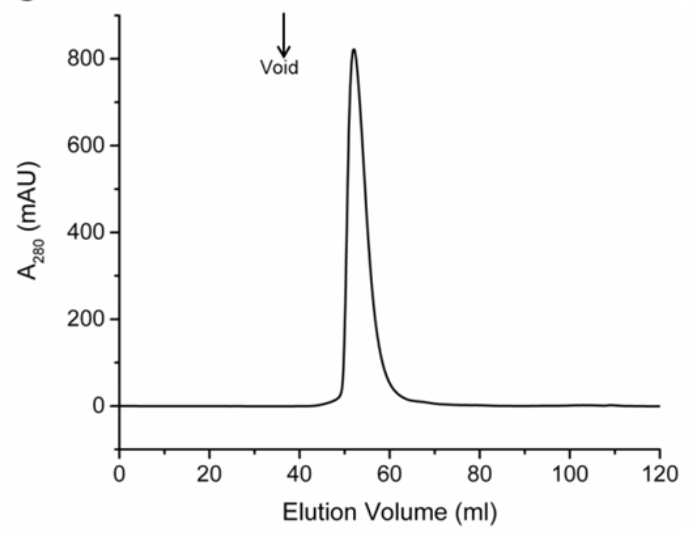

B
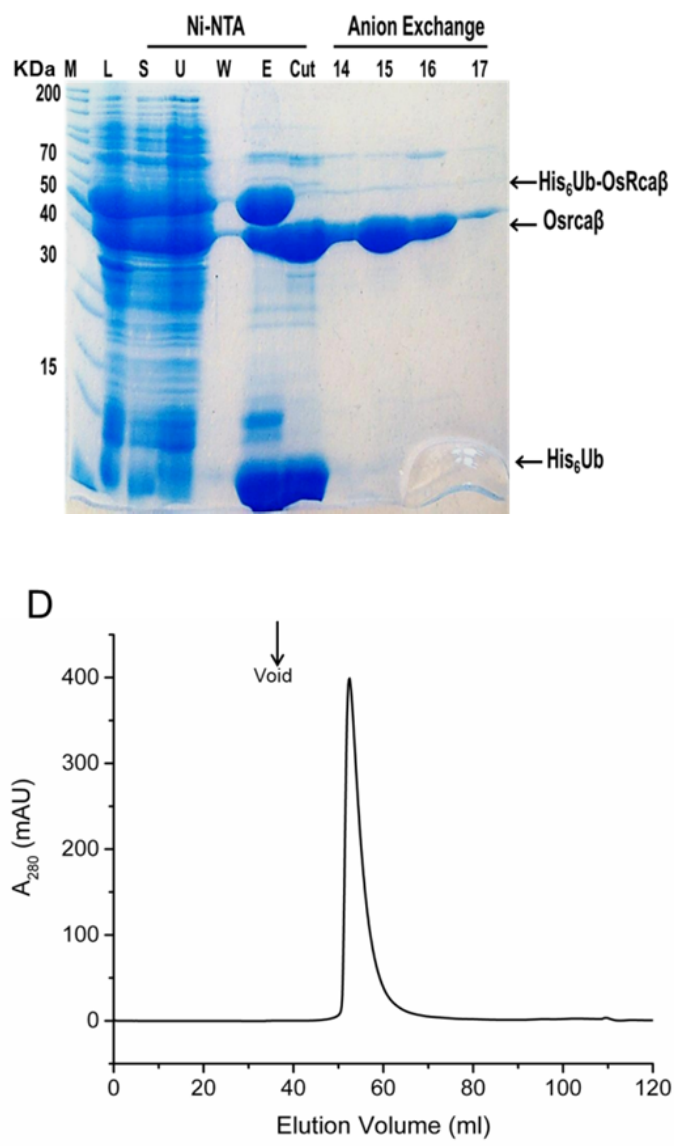


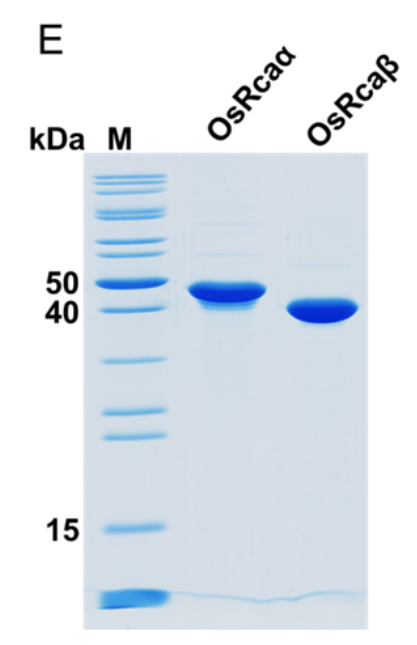

Fig. 3.1: Representative purification of OsRcad and OsRcaß. (A and B) 12.5\% SDS-PAGE analysis. Successive purification by IMAC (Ni-NTA), followed by USP2 mediated cleavage of Nterminal tag and anion exchange chromatography (AIEC) using a MonoQ 10/100 column yielded pure protein. (M: Molecular Weight Marker, L: Lysate, S: Soluble, Ni-NTA: Fractions after IMAC, U: Unbound, W: Wash, E: Final eluate, Cut: eluate after USP2 mediated cleavage of Ubiquitin. The numbers indicates the SDS samples from respective fractions of AIEC). (C and D) Size exclusion chromatograms of OsRca $\alpha$ and OsRca $\beta$ respectively using a Superdex 200 16/600 column showing a single peak of each Rca purified. (E) Final purified protein on 16\% SDS-PAGE gel.

A representative illustration of the purification of Rca isoforms is shown in Fig. 3.1. Rca isoforms were captured by immobilized metal ion affinity chromatography (IMAC) followed by precise cleavage of $\mathrm{His}_{6}$-Ubiquitin from the $\mathrm{N}$-terminus of the expressed Rca by ubiquitin specific protease (USP2). Overnight cleaved protein was subjected to anion exchange chromatography (AIEC) to yield highly pure protein as analyzed by SDS-PAGE. Whenever deemed necessary, further purification was performed by size exclusion chromatography (Fig. 3.1C and D). The average yield of protein from 11 culture of E.coli BL21 grown in LB media after induction with IPTG at $23{ }^{\circ} \mathrm{C}$ was found to be $\sim 15-18 \mathrm{mg}$ of pure protein.

\subsubsection{Purification of Oryza sativa thioredoxin-f}

Thioredoxin-f of rice (OsTrx-f) was heterologously expressed in E. coli BL21 as a Nterminal fusion with $\mathrm{His}_{6} \mathrm{Ub}$ similar to that described for the activases above. 
A

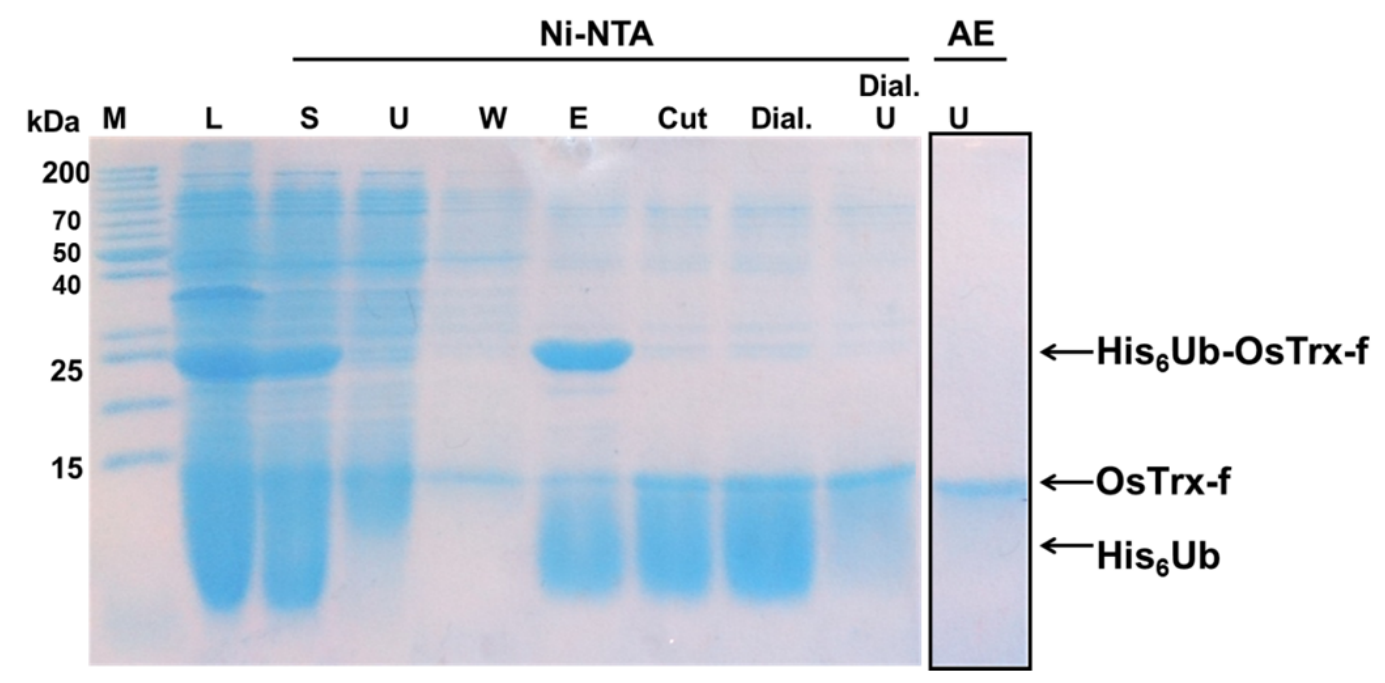

B

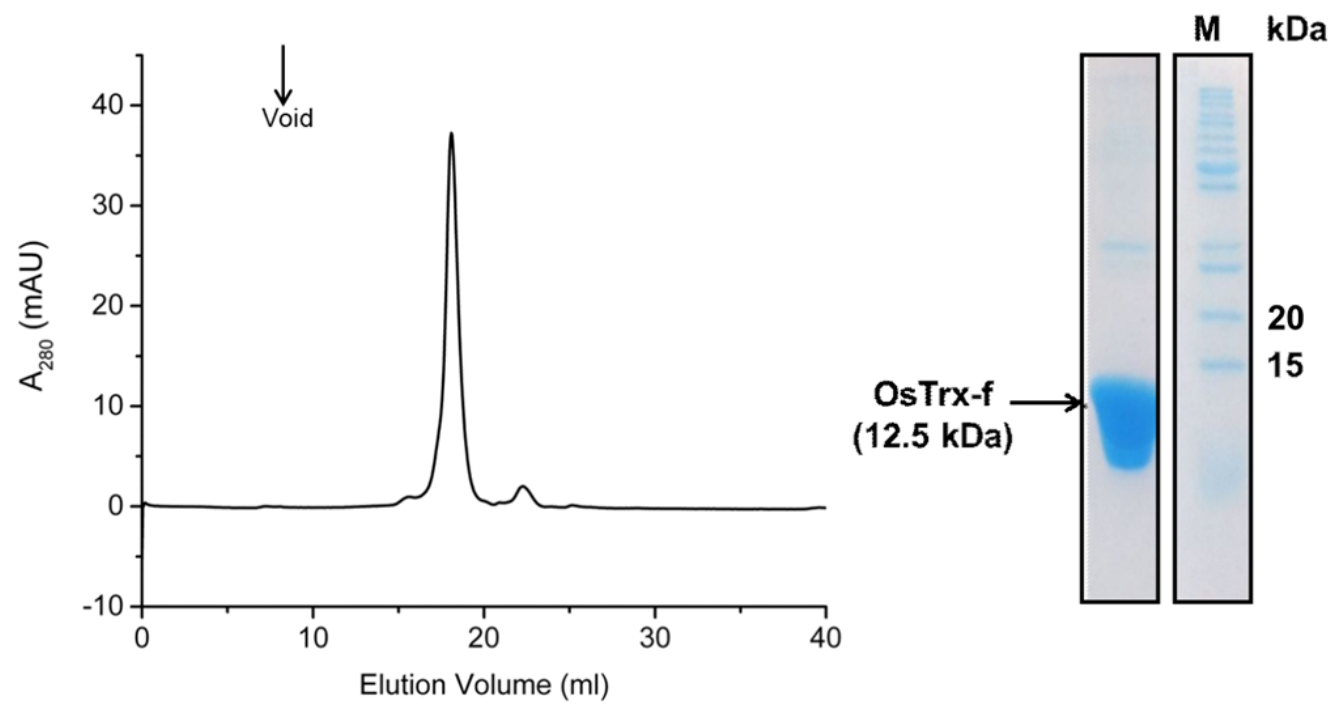

Fig. 3.2: Representative purification of OsTrx-f. (A). 16\% SDS-PAGE analysis. Successive purification by IMAC (Ni-NTA), followed by Usp2 mediated cleavage of N- terminal tag, dialysis and anion exchange chromatography (AIEC) using a MonoQ column. (M: Molecular Weight Marker, L:Lysate, S:Soluble, Ni-NTA: Fractions after IMAC, U: Unbound, W:Wash,: E: Final eluate, Cut : eluate after USP2 mediated cleavage of Ubiquitin., Dial.: fraction after dialysis, Dial. U: unbound fraction from Ni-NTA after dialysis. (B). Size exclusion chromatogram of OsTrx-f using a Superdex $20010 / 300$ column shows a single peak of purified thioredoxin-f (left). Final purified protein on 16\% SDS-PAGE (right).

The protein was purified by IMAC using Ni-NTA resins followed by cleavage by USP2 and dialyzed in buffer containing low imidazole concentration $(20 \mathrm{mM}$ Tris$\mathrm{HCl} \mathrm{pH} 8,50 \mathrm{mM} \mathrm{NaCl}$ and $10 \mathrm{mM}$ imidazole) to remove excess imidazole. The 
protein solution was passed back to Ni-NTA column equilibrated with dialysis buffer to separate the His 6 -ubiquitin. The flow through was collected as His 6 -ubiquitin would bind to the column and analyzed by 16\% SDS-PAGE as shown (Fig. 3.2A). Further purification was done by anion exchange chromatography, however, protein was not found to bind onto the column and collected as flow through which was passed again to the Ni-NTA column to finally yield $\mathrm{His}_{6}$-ubiquitin free protein. The protein was further purified using size exclusion chromatography yielding a single peak (Fig $3.2 \mathrm{~B})$.

\subsubsection{Purification of Oryza sativa Rubisco}

Oryza sativa Rubisco was directly purified from 10-12 week old plant leaves as described previously [188] and in methods (section 2.7.8). Ammonium sulphate precipitation at $33 \%$ and $55 \%$ was performed. Rubisco was found to be precipitated at $55 \%$ ammonium sulphate salt in the pellet. Further steps included the desalting of protein using dialysis in a low salt buffer followed by anion exchange chromatography which yielded highly pure protein as analyzed by SDS-PAGE (Fig. 3.3A). Whenever necessary, further purification was carried out by size exclusion chromatography (Fig. 3.3B). A typical yield from $8 \mathrm{~g}$ of fresh rice leaf material was found to be $\sim 10-12 \mathrm{mg}$ of pure protein. 
A

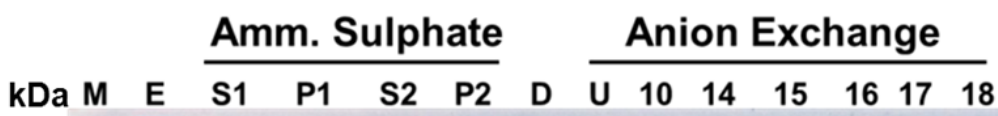
200

70

B

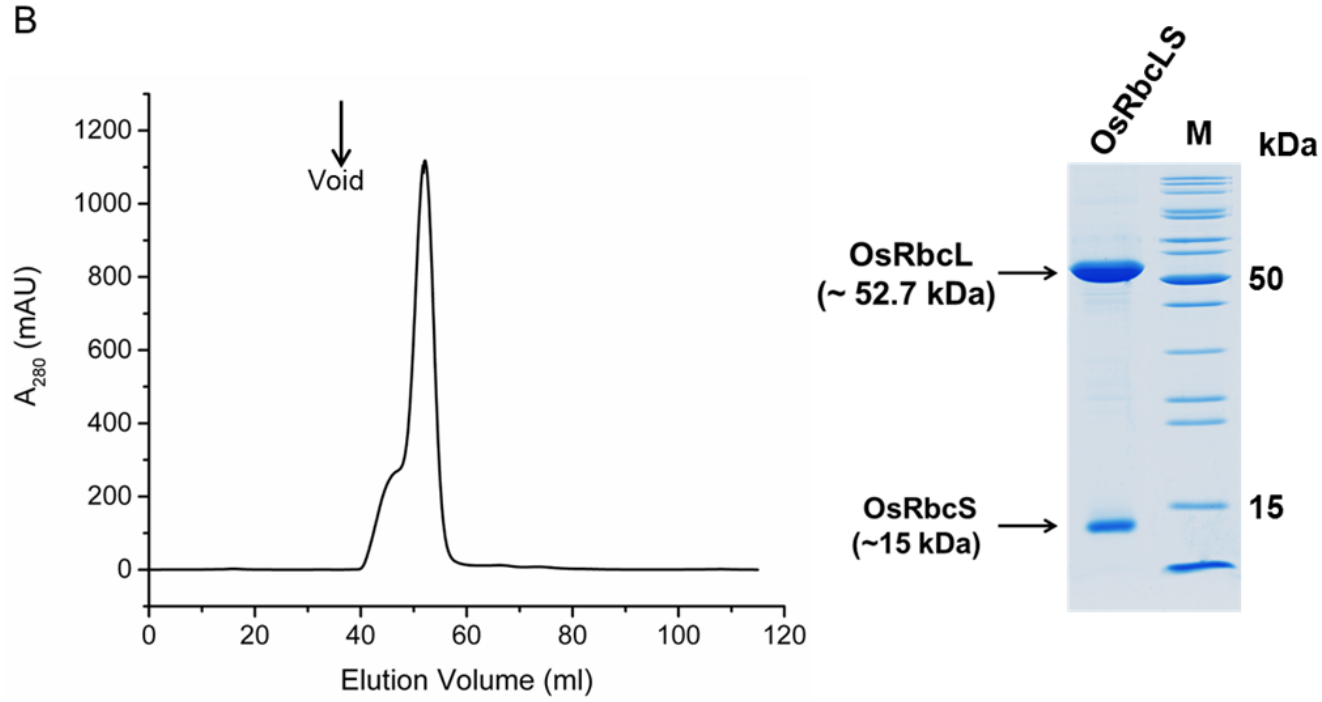

Fig. 3.3: Representative purification of Rubisco. (A) $16 \%$ SDS-PAGE analysis. Successive purification by ammonium sulphate precipitation, followed by dialysis and anion exchange chromatography (AIEC) yielded pure protein. (M: Molecular Weight Marker,E: Extract, S:Soluble fraction after ammonium sulphate precipitation: Pellet after ammonium sulphate precipitation., D: Dialyzed fraction, U: Unbound fraction on MonoQ. The numbers indicates the SDS samples from respective fractions of AIEC on a MonoQ 10/100 column. (B) Size exclusion chromatogram of Rubisco on a Superdex 200 16/600 column (left). Purified fractions on a 16\% SDS-PAGE clearly indicating large and small subunits of Rubisco (right).

\subsection{Recombinant Rca isoforms were biochemically active and can activate inhibited rice Rubisco}

In order to test the activity of Rubisco activases to be used further in the study, the biochemical activities of the rice Rca were assessed by their ability to activate inhibited Rubisco complex and hydrolyse ATP. Thus, Rubisco activation as well as 
ATPase activity assays were performed using continous enzyme-coupled spectrophotometric assays at $25^{\circ} \mathrm{C}[189,190]$ as described in section 2.7.10.

The activated carbamylated Rubisco (ECM) complex was generated by incubating 20 $\mu \mathrm{M}$ Rubisco active sites with $20 \mathrm{mM} \mathrm{NaHCO}{ }_{3}, 10 \mathrm{mM} \mathrm{MgCl} 2$ in buffer A (Table 2.4). The RuBP inhibited Rubisco (ER) complex was made in the same buffer by incubating $20 \mu \mathrm{M}$ Rubisco active sites with $4 \mathrm{mM}$ EDTA to chelate free magnesium ions for $10 \mathrm{~min}$ at $25{ }^{\circ} \mathrm{C}$. After this, $1 \mathrm{mM} \mathrm{RuBP}$ was added to make the final ER complex. The Rubisco activity was then measured using the spectrophotometric assay at $25{ }^{\circ} \mathrm{C}[190]$ in presence of $20 \mathrm{mM} \mathrm{NaHCO}_{3}$ and $5 \mathrm{mM} \mathrm{MgCl}_{2}$ in the final reaction mixture (section 2.7.10). The concentrations of Rubisco and activase were chosen within the subsaturating limits at $0.5 \mu \mathrm{M}$ ECM or ER (active sites) and $5 \mu \mathrm{M}$ activase protomer. The assay monitors the continous oxidation of NADH leading to a reduction in absorbance at $340 \mathrm{~nm}$. This reduction in absorbance is directly coupled to production of 3-phosphoglycerate (3PG) and can be plotted against time to determine the activity of Rubisco in presence and absence of activase (Fig. 3.4). The 3PG values after particular time interval could be derived from the absorbance values using the following equation:

$$
3 \mathrm{PG}(\mu \mathrm{M})=-\left(\mathrm{A}_{\mathrm{T}}-\mathrm{A}_{0}\right) / 0.00622 / 2
$$

Where, $A_{T}$ and $A_{0}$ are the absorbance values at time $T$ and zero and 0.00622 is extinction coefficient of NADH in $\mu \mathrm{M}^{-1} \mathrm{~cm}^{-1}$. Since formation of one $3 \mathrm{PG}$ required oxidation of two NADH in the assay (see scheme in section 2.7.10.2), the amount of 3PG produced will be half of NADH oxidized [190].

Since, production of two $3 \mathrm{PG}$ utilizes consumption of one RuBP, the rate of $\mathrm{CO}_{2}$ fixed or rate of ECM could be determined by:

$$
\text { Rate of ECM }\left(\mathrm{V}_{\mathrm{ECM}}\right)=\mathrm{RuBP} \text { consumed } \mathrm{s}^{-1} / \text { concentration of } \mathrm{ECM}
$$

Where, RuBP consumed will be half of 3PG produced in equation (1) as per assay stoichiometry [190]

The fully activated rice ECM fixed $\mathrm{CO}_{2}$ at a rate of $0.72 \pm 0.10 \mathrm{~s}^{-1}$ (rate of ECM) (from Fig. 3.4A data). RuBP inhibited complex (ER) also possess residual Rubisco activity 
in absence of activase. However, the rate of $3 \mathrm{PG}$ production was negligible compared to ECM (Fig. 3.4A).

The Rubisco activase activity was determined using the method of Lan and Mott [198] without fitting the polynomial function to the time course of the Rubisco activity assay. Instead the gradient between the two time points in the data could be directly utilized to obtain the first derivative of the curves. The concentration of ECM was then calculated using the equation below and plotted against time.

$$
[E C M]=-\left[\left(\mathrm{A}_{2}-\mathrm{A}_{1}\right) /\left(\mathrm{T}_{2}-\mathrm{T}_{1}\right) / 0.00622\right] / \mathrm{NADH} \text { oxidized } / \mathrm{V}_{\mathrm{ECM}}
$$

Where, $\left(A_{2}-A_{1}\right) /\left(T_{2}-T_{1}\right)$ is the gradient between two time points, and 0.00622 is extinction coefficient of NADH in $\mu \mathrm{M}^{-1} \mathrm{~cm}^{-1}$, NADH oxidized for one second will be 4 , thus the value of it will be depend on the difference in time point ( 1 RuBP $=$ $2 * 3 \mathrm{PG}=4 \mathrm{NADH})[190]$.

A linear function was then fitted through the origin to obtain the rate of ECM formation. The obtained rate of ECM in presence of activase was normalized by substracting the residual rate of ECM formation by ER complex in absence of activase (Fig. 3.4B) to quantitate actual activase activity for total Rca protomer in reaction $(5 \mu \mathrm{M})$. To obtain the activity per $\mu \mathrm{M}$ Rca protomer in $\mathrm{nM} E C M \min ^{-1}$ as shown in Fig. 3.4C, the normalized values were first divided by 5 and then multiplied by a factor of $1000 * 60$ (conversion factor for $\mu \mathrm{M}$ to $\mathrm{nM}$ and second to minute)

Both isoforms of OsRca were found to be capable of activating RuBP inhibited Rubisco active sites (ER) and hydrolyzing ATP but at variable rates (Fig. 3.4A and C). The short isoform (OsRca $\beta)$ was found to be significantly faster ( 2.5-3 fold) in activating ER complex than the long isoform (OsRca $)$. In terms of ATPase activity also, OsRca $\beta$ possessed $\sim 3$-fold higher ATPase rate than OsRca $\alpha$ under assay conditions. Consistent with these findings, a recent study [177], also reported a comparable trend, where OsRcaß displayed more than 2-fold difference in terms of Rubisco activation and ATPase activity under similar concentration of activase. 
A
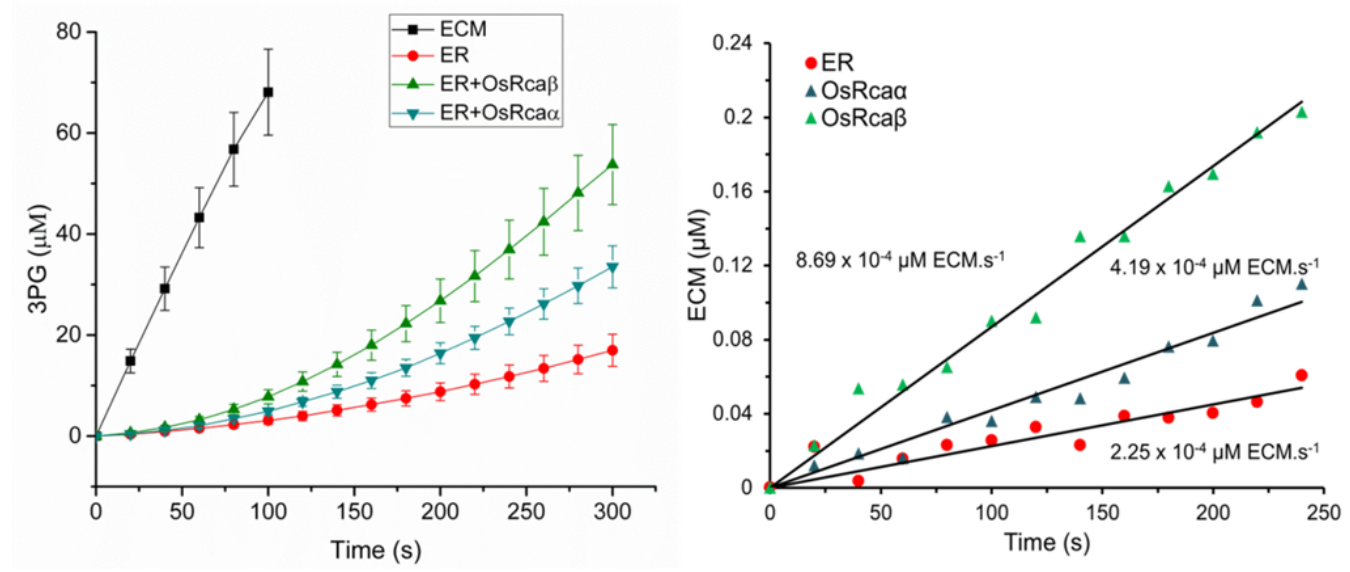

B

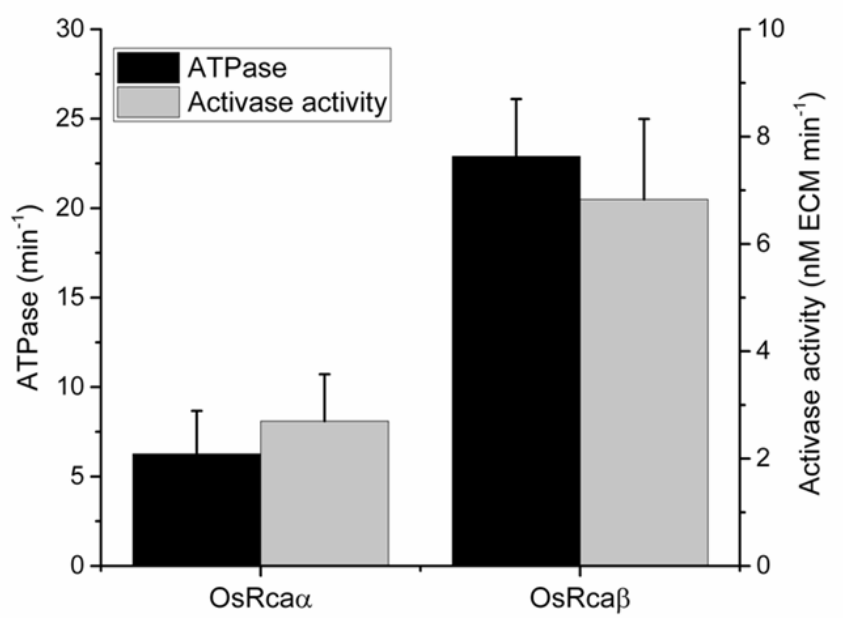

Fig. 3.4: Biochemical properties of Rubisco activase isoforms from Oryza sativa. (A) Rubisco activation assay of RuBP-inhibited Rubisco (ER) of rice by its Rca isoforms performed at $25^{\circ} \mathrm{C}$ with $0.5 \mu \mathrm{M}$ Rubisco active sites and $5 \mu \mathrm{M}$ activase protomer (left). Representative quantification of activase activity. ECM concentration was calculated using the gradient between two time points and plotted against time. A linear function was fitted through the origin to obtain the activase activity (right). (B) ATPase activity assays $\left(5 \mu \mathrm{M}\right.$ Rca protomer, $\left.25^{\circ} \mathrm{C}\right)$ and quantified Rubisco activase activities of WT Rca isoforms. Error bars indicate the standard deviations of mean values of three or more independent experiments.

\subsection{Redox regulation of the OsRca in the presence of thioredoxin-f}

The low activity of OsRca $\alpha$ we observed above was likely attributable to the fact that Rubisco activase in most plant species is regulated via changes in the redox state of two cysteine residues located at C-terminal extension of $\alpha$ isoform [146]. The redox state is modulated by the electron carrier thioredoxin- $f$, thus providing a mechanism for the light regulation of Rubisco in vivo [150, 199, 200]. 


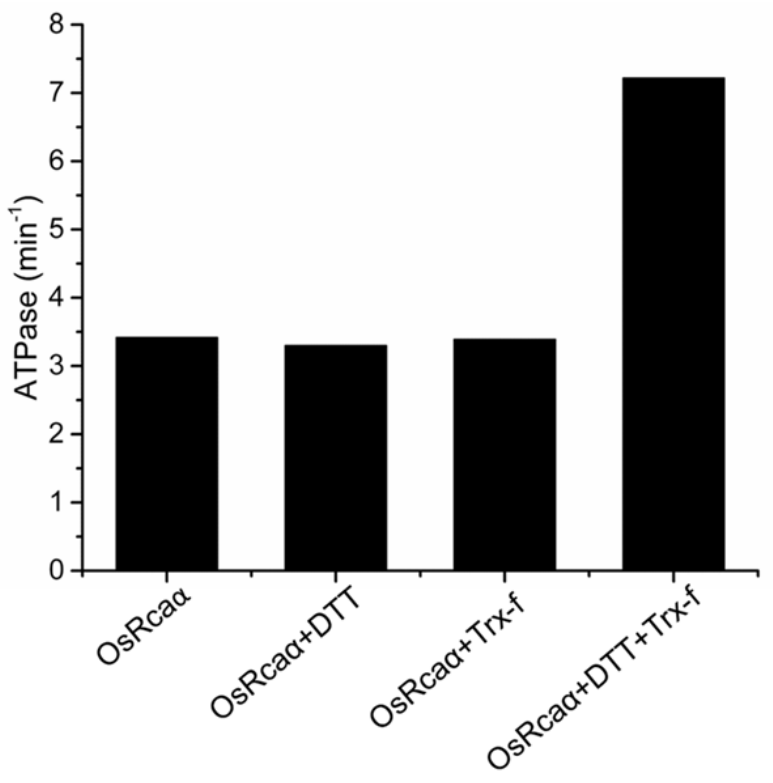

Fig. 3.5: ATPase activity of OsRcaa was stimulated in presence of DTT and thioredoxin -f. (A) Effect of thioredoxin-f on the ATPase activity of OsRca $\alpha$. OsRca $\alpha$ ( $25 \mu \mathrm{M}$ protomer) was preincubated with $5 \mathrm{mM}$ dithiothreitol (DTT) and $3.6 \mu \mathrm{M}$ rice thioredoxin- $\mathrm{f}$ (Trx-f) for 10 min before the measurement. Assays were performed at $25{ }^{\circ} \mathrm{C}$ with $5 \mu \mathrm{M}$ OsRca, $1 \mathrm{mM}$ DTT and $0.72 \mu \mathrm{M}$ rice Trx-f in the final reaction mix.

Under in vitro conditions, activity of $\alpha$ isoform could be modulated by changing the redox state in the presence of DTT and thioredoxin-f as shown (Fig. 3.5). It was observed that the ATPase activity of the isoform of rice was increased almost two fold in presence of DTT and thioredoxin-f. The redox study performed earlier with $\alpha$ isoform of arabidopsis reported 50\% increase in ATPase activity after treatment with DTT and thioredoxin-f [146]. However, DTT and thioredoxin alone were not capable of providing necessary reduction (Fig. 3.5) and works like a reductive relay where DTT acts as electron donor for thioredoxin to function as provided by photosystem-I in vivo [151].

\subsubsection{Evidence for covalent complex formation between OsRca $\alpha$ and thioredoxin-f}

Reduction of substrate takes place via formation of a transition state heterodisulfide between thioredoxin-f and substrate due to disulphide reduction by reduced thioredoxin [201]. It is possible to trap this interaction due to formation of a stable covalent heterodimers by generating a mutant of thioredoxin-f which replaces one of 
the active site cysteine involved in formation of intermolecular mixed disulfide intermediate [202, 203]. Therefore, we replaced one cysteine of rice thioredoxin-f to serine (C42S), which should permit formation of a mixed disulfide between thioredoxin-f and Rca [204].
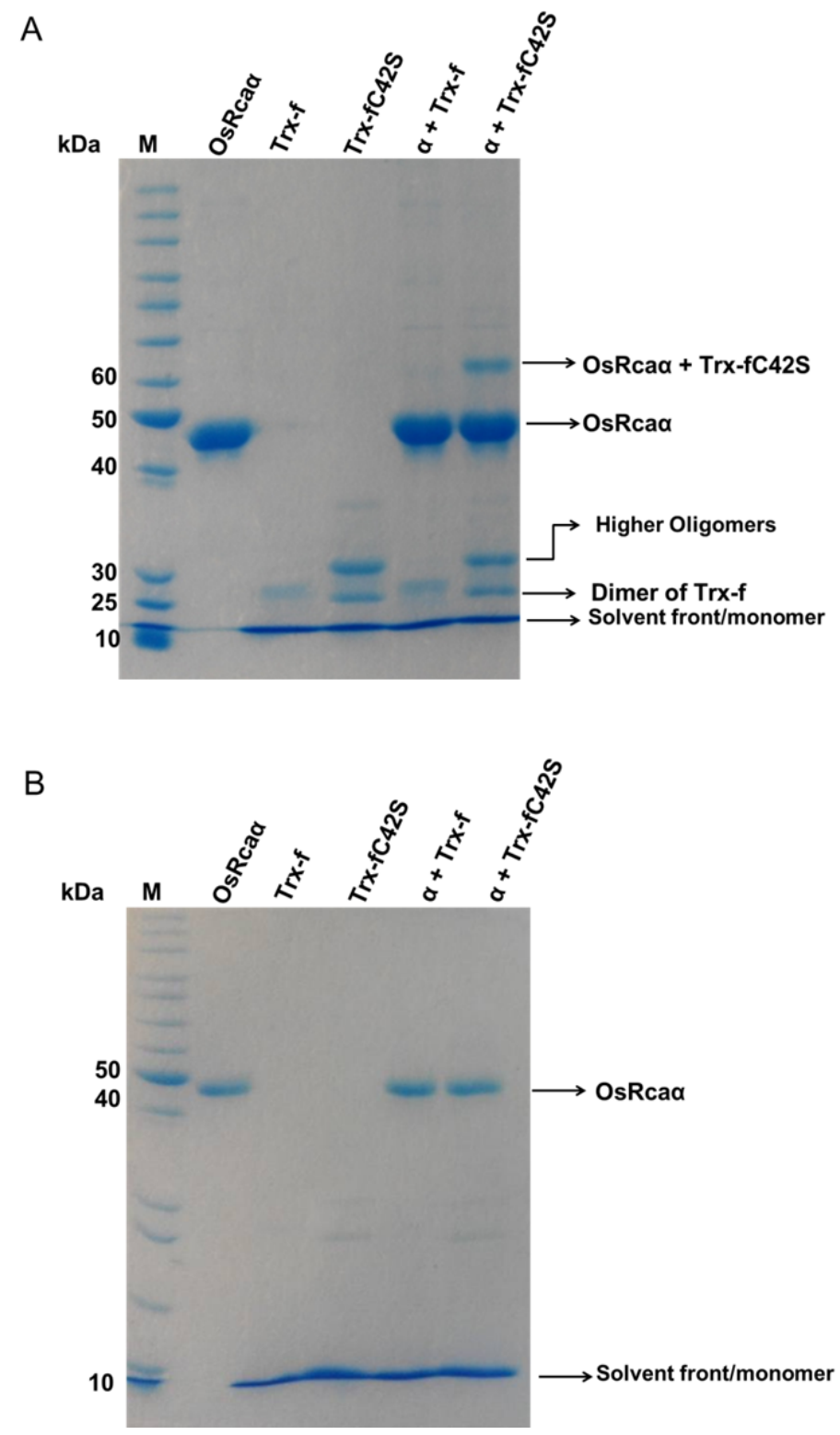

Fig. 3.6: SDS-PAGE analysis of the reaction mixtures containing OsRcao and Trx-f. (A) The two

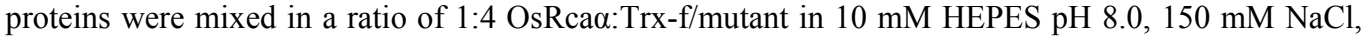
and incubated for $30 \mathrm{~min}$ at $25^{\circ} \mathrm{C}$ under aerated environment. Aliquots of the incubated mixtures were then separated on $10 \%$ SDS- PAGE gel under non-reducing condition without heat treatment. (OsRca $\alpha$ or $\alpha$ : $\alpha$ isoform of rice Rubisco activase, Trx-f: thioredoxin-f). (B) 12.5\% SDS-PAGE gel of the similar samples as A under reducing condition in presence of DTT. 
In order to obtain a stable disulphide mixed intermediate between OsRca $\alpha$ and Trx-f the two proteins were mixed in excess of Trx-f and its mutant (C49S) with OsRca $\alpha$ (1:4) under aerated condition for $30 \mathrm{~min}$ at room temperature and analyzed using SDS-PAGE. For control, all the proteins were run in isolation. The complex formation was only obtained in presence of the Trx-f mutant, while no complex formation was seen in case of WT Trx-f (Fig. 3.6A). There is formation of additional homo-dimers of Trx-f under non-reducing conditions, which were reversible in nature and could be easily eliminated under reducing conditions in the presence of DTT as reported previously (Fig. 3.6B) [204-206]. This was consistent with the reported structural and functional form of oxidised thioredoxin- $\mathrm{f}[207,208]$. Also, equilibrium binding study have revealed the evidence for dimer formation and its binding to chloroplastic FBPase in pea [209].

In case of thioredoxin mutant $(\mathrm{C} 42 \mathrm{~S})$, we observed an additional band above the dimer band (Fig. 3.6A). The formation of homo-dimer and higher molecular mass protein has been reported earlier for thioredoxin-f of tobacco for recombinant protein as well in planta under various non-reducing conditions, especially in presence of high salt [205]. It has also been reported that these dimers could be separated by high ionic strength into monomers [207]. The evidence presented for the formation of the stable complex between Rca $\alpha$ and Trx-f in this study may facilitate further structural and biochemical studies. For instance, a structural and functional understanding of the specificity between different thioredoxin isoforms and their substrate is still elusive $[210,211]$. Recent advancements in cryo-EM technology should permit characterization of such complexes at atomic resolution.

\subsection{Characterization of Rubisco activase thermostability}

A relatively low thermal stability causes denaturation of Rca at moderately elevated temperature which is believed to be directly responsible for shutting down photosynthesis in vivo [11]. However, the biophysical properties of activase vary between species with the Rca $\alpha$ reported to be more thermostable and conferring thermostability to Rca $\beta$ in spinach [141, 178], but not in arabidopsis [166]. Importantly, heat stress in rice leads to upregulation of Rca $[196]$ and it was reported to be the most upregulated protein in Oryza meridionalis [197]. However, such 
difference in relative abundance was not observed in arabidopsis under heat stress [212]. Thus, we performed a thermal characterization of both rice Rca isoforms.

\subsubsection{Rice Rubisco activases are also relatively thermolabile}

Fig. 3.7 shows the ATPase activity of OsRca $\alpha$ and OsRca $\beta$ isoforms after a $10 \mathrm{~min}$ heat treatment at various temperatures. It was found that in terms of activity, both isoforms possessed similar thermal stability and started losing their activity above 35 ${ }^{\circ} \mathrm{C}$. While, OsRca $\beta$ lost $50 \%$ of its maximum activity at $40{ }^{\circ} \mathrm{C}$, OsRca $\alpha$ lost $50 \%$ of its maximum activity at $37^{\circ} \mathrm{C}$ and was completely inactivated at $40^{\circ} \mathrm{C}$. OsRca $\beta$ lost most of its activity at $42{ }^{\circ} \mathrm{C}$ and became completely inactive at $45^{\circ} \mathrm{C}$. A decline in ATP hydrolysis rate was also reported in the recent study performed on rice activases after temperature exceeded $36-40{ }^{\circ} \mathrm{C}$ [177].

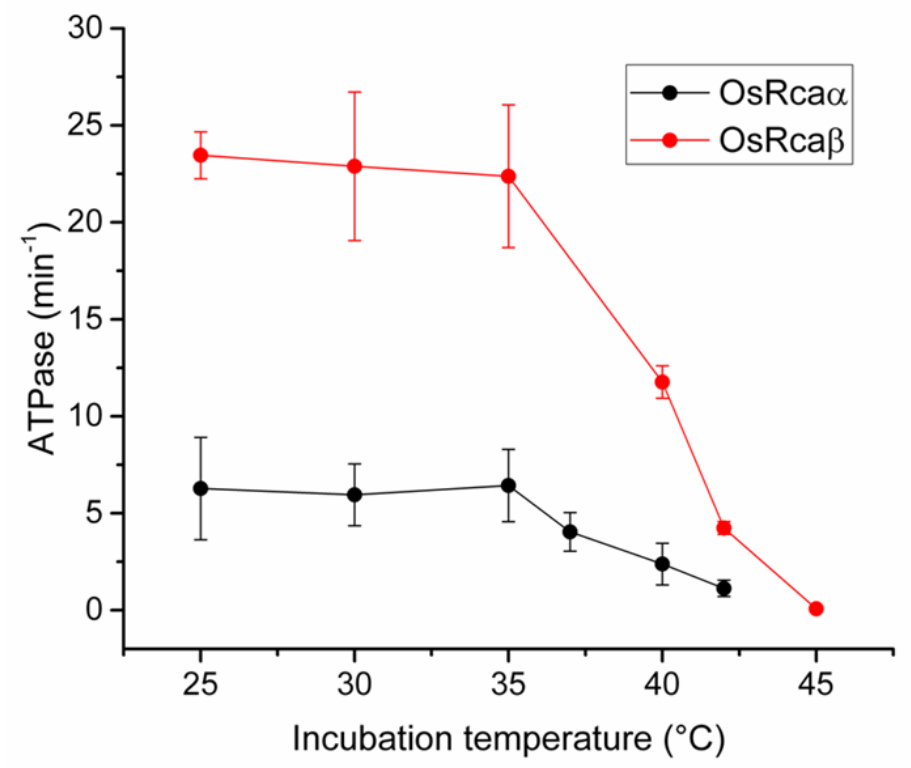

Fig. 3.7: Decline in ATPase Activity of Rubisco activase upon heat treatment. The activase isoforms $(260-280 \mu \mathrm{M})$ were pre-incubated for $10 \mathrm{~min}$ at indicated temperature. ATPase activity was then measured at $25^{\circ} \mathrm{C}$ with $5 \mu \mathrm{M}$ Rca (protomer) in the final reaction mix. Error bars indicate the standard deviation of mean values of three or more independent experiments.

To determine the structural integrity of Rubisco activase at different temperatures, the molar ellipticity of protein samples was determined by circular dichroism (CD). Experiments were performed and spectra were collected as described in materials and methods (section 2.7.6). 
The secondary structural elements could be estimated by their unique spectral pattern. For instance, $\alpha$-helices are characterized by a depression at 222 and $208 \mathrm{~nm}$ and an elevation at $193 \mathrm{~nm}$. On the other hand, $\beta$-sheets could be identified by a negative bands at $218 \mathrm{~nm}$ and a positive band at $195 \mathrm{~nm}$ [213]. The secondary structural content of Rca was determined by using the K2D2 algorithm [214]. At 20-25 ${ }^{\circ} \mathrm{C}$, OsRca $\alpha$ and $\beta$ predominantly possessed high $\alpha$ - helical content (56 and 58\% respectively), while $\beta$-sheet content was estimated to be $12 \%$ and $7 \%$ respectively. The CD spectra reported for the $\beta$-isoform of arabidopsis estimated $57-61 \% \alpha$-helix and $7-10 \% \beta$ sheet at $15{ }^{\circ} \mathrm{C}$ [134], while tobacco contained $44 \% \alpha$-helix and around $10 \% \beta$-sheet at $20{ }^{\circ} \mathrm{C}[158]$. At 45 or $50{ }^{\circ} \mathrm{C}$, rice $\alpha$-isoform possessed only $8 \% \alpha$ helixes, while the $\beta$-isoform retained $18 \% \alpha$-helixes. Earlier study on $\beta$-isoform of arabidopsis reported $8-22 \% \alpha$-helical content at $65{ }^{\circ} \mathrm{C}[134]$. Thus, the majority of $\alpha$ helical content was lost at high temperature consistent with denaturation of the protein.

The mid-point of thermal denaturation was approximately determined by visual inspection of the observed ellipticity versus temperature plot at $208 \mathrm{~nm}$ (Fig. 3.8) $[134,138]$. We observed, the mid-point for thermal denaturation (apparent $\mathrm{T}_{\mathrm{m}}$ ) lies at about $35-40{ }^{\circ} \mathrm{C}$ for both rice Rubisco activase isoforms. This was similar to the earlier report for arabidopsis $\beta$-isoforms, where the apparent $T_{m}$ was estimated to be about 40 ${ }^{\circ} \mathrm{C}$ using $\mathrm{CD}$ and mid-point for loss of activity at around $37^{\circ} \mathrm{C}[134]$. Based on such observations, it was concluded that the loss of activase function is associated with structural instability of Rca at elevated temperature [134]. Under identical incubation conditions (temperature), the ATPase activity of Rubisco activase decreased with increasing temperature, exhibiting a mid-point of about $37{ }^{\circ} \mathrm{C}$ and $40{ }^{\circ} \mathrm{C}$ for $\alpha$ and $\beta$ isoform respectively as shown in previous section. This clearly indicates that loss of activity of activase is due to the loss of secondary structure and denaturation of Rca at high temperature which was further confirmed by native PAGE analysis (Fig 3.9A) of the Rca isoforms. 

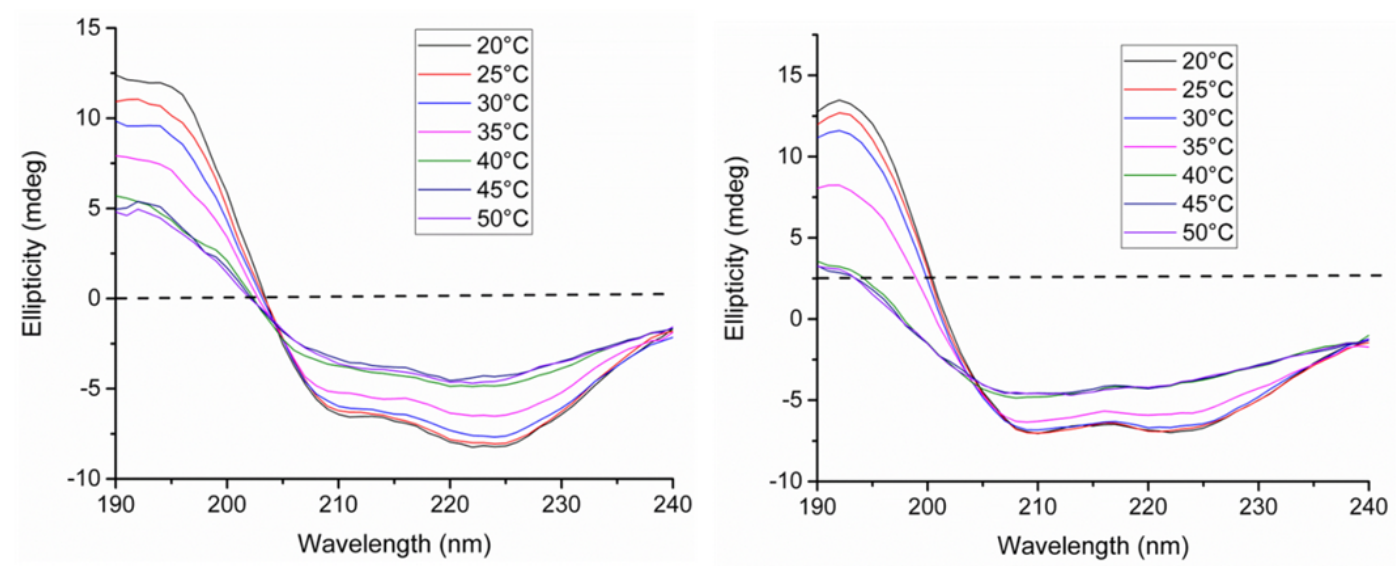

C

$\mathrm{D}$
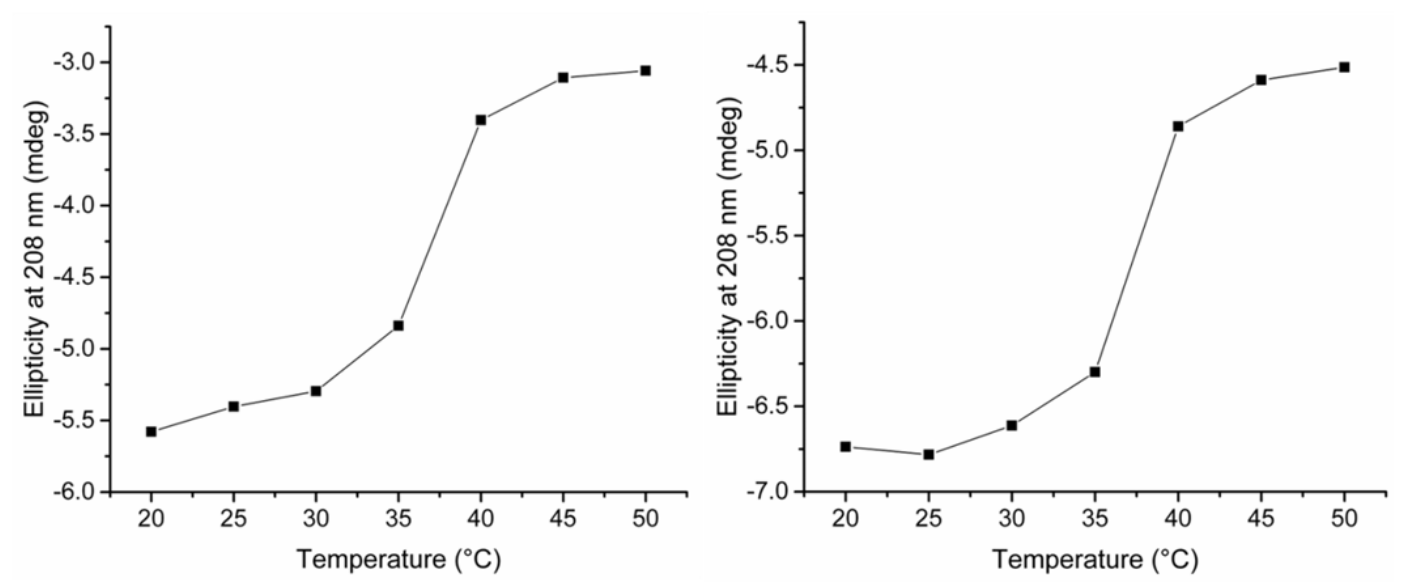

Fig. 3.8: CD spectroscopic profile of Rubisco activases at different temperature. (A and B) CD spectra of OsRca $\alpha$ and $\beta(1 \mathrm{mg} / \mathrm{ml})$ respectively recorded at different temperature $\left(20-50{ }^{\circ} \mathrm{C}\right)$. (C and D) Ellipticity of OsRca $\alpha$ and $\beta$ respectively monitored at $208 \mathrm{~nm}$ as a function of temperature derived from the CD curves.

We observed that at temperature $40{ }^{\circ} \mathrm{C}$ or above, Rca appears to form higher ordered aggregates which migrate more slowly on a 6\% Native PAGE gel. However, SDSPAGE and turbidity analysis, along with visual inspection of the Rca protein solution shows that although there is partial loss of activity at temperature above $35{ }^{\circ} \mathrm{C}$ but rice activase isoforms remain in solution and form soluble inactive aggregates (Fig. 3.9B and C). Contrary to this, tobacco Rca gets highly turbid when heated at $45{ }^{\circ} \mathrm{C}$ which was easily discernible. This was consistent with the earlier study performed on tobacco, where a rapid increase in light scattering was reported when temperature was increased above $40{ }^{\circ} \mathrm{C}$ [215]. Another study on leaf tissue of wheat and cotton also demonstrated formation of insoluble higher molecular mass aggregates at similar 
temperature using western blot analysis [216]. Although no precipitation was observed for rice activase isoform, ATPase activity was completely lost after heat treatment at such temperature (Fig. 3.7). The previous results from Barta et al (2010) and present data suggested that like in arabidopsis, in rice also, denaturation at high temperature leads to production of soluble inactive higher order aggregates. Should this behaviour extend to the in vivo systems, it may indicate different mechanisms of Rca reactivation. For instance, smaller soluble species should be easier to recover than aggregated, inclusion like forms.

A

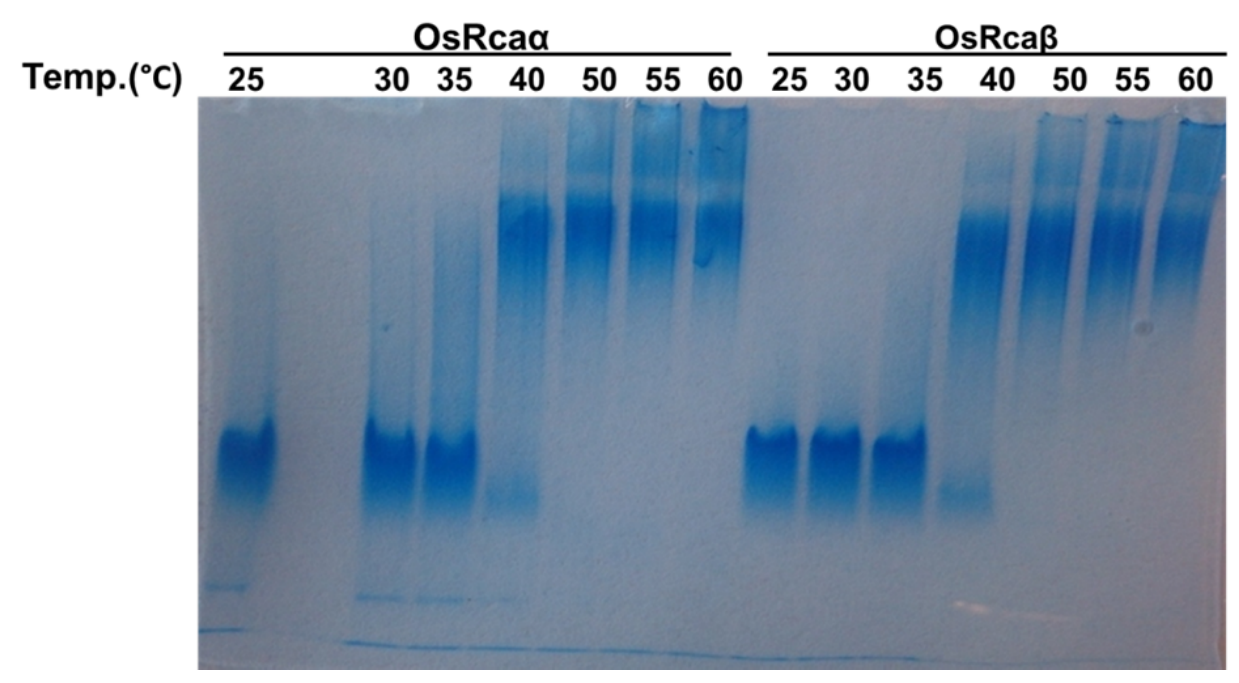

B

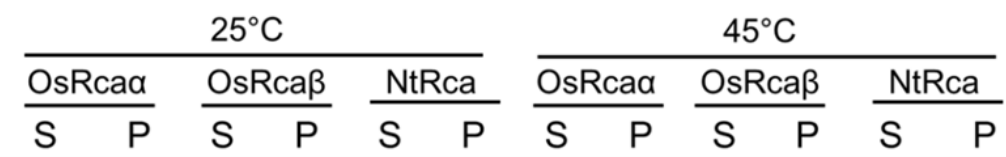

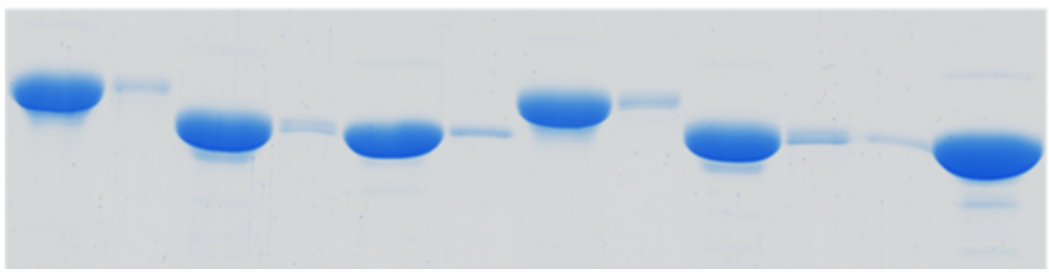




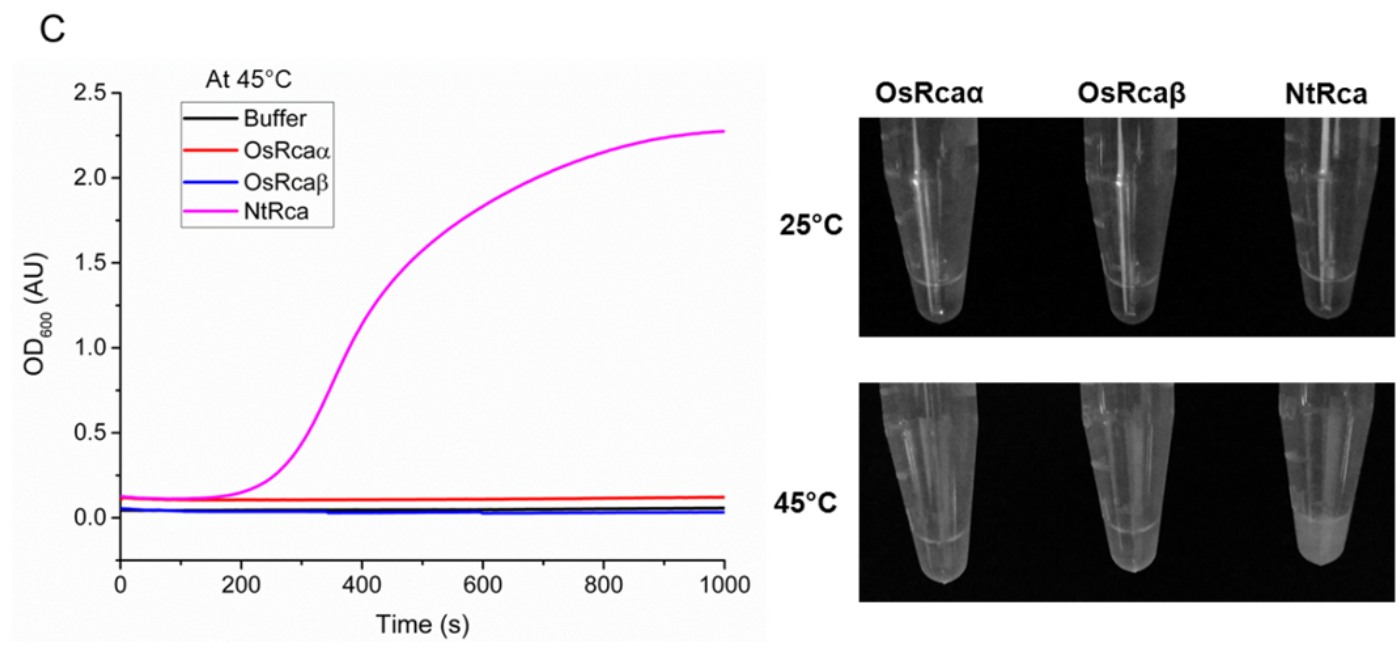

Fig. 3.9: Thermostability analysis of Rubisco activase. (A) $6 \%$ Native PAGE of Rca at different temperature. Rca $(3 \mathrm{mg} / \mathrm{ml})$ was heated at the respective temperature for $10 \mathrm{mins}$ and $10 \mu \mathrm{g}$ of final Rca was loaded on to the gel. (B) 12.5\% SDS-PAGE analysis of the soluble (S) and pellet (P) fraction OsRca and NtRca (tobacco) after incubating at respective temperature. (C) Turbidity analysis of the Rca at $45^{\circ} \mathrm{C}$. Rice (OsRca) and tobacco (NtRca) activase were diluted to $3 \mathrm{mg} / \mathrm{ml}$ and optical density $(600 \mathrm{~nm})$ scan against time was performed at $45^{\circ} \mathrm{C}$ (left). Activase samples $(3 \mathrm{mg} / \mathrm{ml})$ were incubated at indicated temperature (right) where rice activase isoforms stay in solution while tobacco Rca forms precipitate.

\subsection{Subunit mixing with OsRcad reduces the ATPase activity of the OsRcaß}

In many plants such as arabidopsis and spinach two isoforms are present in equimolar ratio [112] however, in rice, Rca $\beta$ is much more abundant than Rca $\alpha$ under nonstressed condition [144]. Interestingly, heat stress leads to upregulation of Rca in rice and its wild species Oryza meridionalis $[196,197]$. This differential distribution of two Rca isoforms in rice leaves should reflect physiological significance in vivo. We therefore hypothesized that in the oxidized state, OsRca $\alpha$ might regulate the activity of OsRcaß. The ability of Rca to rapidly exchange subunits has been exploited earlier to study the role of various mutations on activase function and subunit coordination [129, 163, 164]. Mixing of wild-type (WT) with ATPase compromised mutants generally have an inhibitory effect on ATPase activity of WT activase. However, the extent of inhibition may vary with the type or site of mutation determining the relative contribution of that residue or region in activase function $[129,163]$. This suggests that it is the nature of inhibition that decides the contribution of particular residue or site towards subunit cooperativity and not just the absence of 
activity of a particular mutant subunit. Therefore, subunit mixing experiments were performed with Rca $\alpha$ and $\beta$ to assess how the interaction of Rca $\alpha$ would affect the activity of the Rca $\beta$ in vitro.

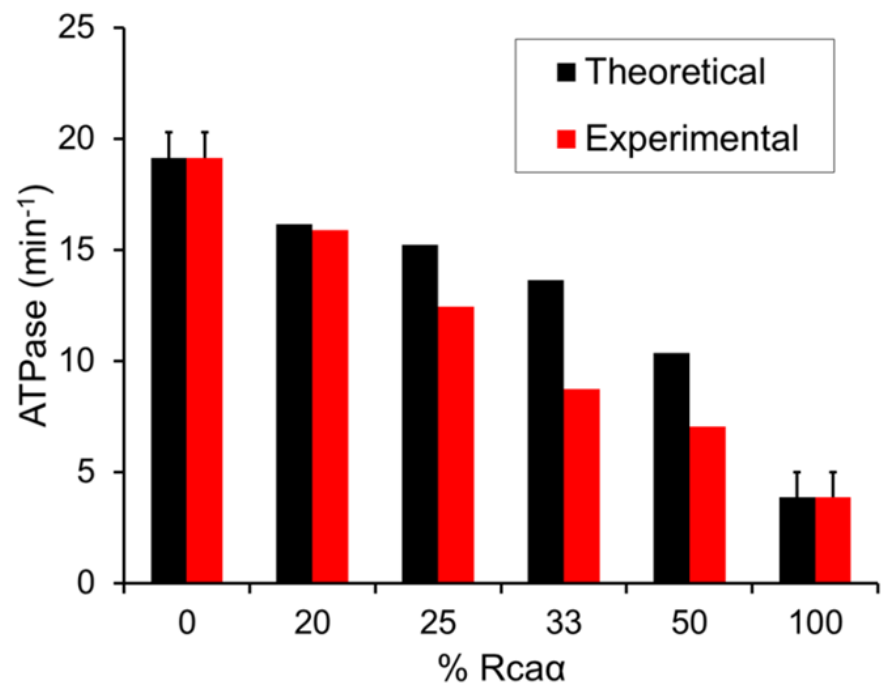

Fig. 3.10: Subunit mixing with OsRca $\alpha$ reduces the ATPase activity of OsRcaß. WT OsRca $\beta$ was pre-incubated with OsRca $\alpha$ at 270-280 $\mu \mathrm{M}$ Rca protomer concentration range for $10 \mathrm{~min}$. ATPase assays were then performed at $25^{\circ} \mathrm{C}$ by keeping the final concentration of Rca $\beta$ as constant $(5 \mu \mathrm{M})$ and gradually varying the concentration of Rca $\alpha$ isoform at different proportion in the final reaction mix as indicated. ATPase is given per Rca active site. The experimental values were calculated using the

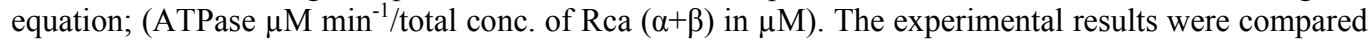
with the theoretical values, assuming subunits behave independently resulting in an additive ATPase rate which can be calculated using the equation; (specific ATPase of Rca $\alpha^{*}$ concentration of Rca $\alpha$ ) + (specific ATPase of Rca $\beta^{*}$ concentration of Rca $\beta$ )/total concentration of Rca $(\alpha+\beta)$ in $\mu \mathrm{M}$.

The preliminary experiment in mixing indicates a reduction in the specific ATPase activity of the Rca mixture when ATPase assay was performed by keeping the concentration of OsRca $\beta$ as constant and titrating up the concentration of OsRca $\alpha$ (Fig. 3.10). Since, OsRca also contributes to the ATPase activity of the heterooligomer, ATPase activity was quantified per active site and therefore the experimental activities were shown in comparison to theoretical activities. Theoretical activities were calculated assuming the subunits were behaving independently and ATPase activity was enumerated as an arithmetic addition of the contribution of individual isoform in the mix based on their specific ATPase activity. We observed that the difference in experimental and theoretical values were not so significant at low proportion $(20 \%)$, however as the proportion of Rca $\alpha$ increased the difference became significant. At the equimolar concentrations (50\%), experimental ATPase 
activity was reduced to $7 \mathrm{~min}^{-1}$, while the theoretical activity was computed as $\sim 11$ $\min ^{-1}$. A lower experimental value than the theoretical one at all proportions clearly suggests the interaction between the two isoform subunits. However, the residual activity of OsRca $\alpha$ complicated the interpretation of the experiment. Thus, performing the above experiment with ATPase inactive mutants of Rca $\alpha$ would provide a better insight.

To further characterize the interaction among Rca subunits, mutations were designed covering key residues responsible for proper functioning of Rca in terms of ATPase activity which have been described in next section.

\subsubsection{Characterization of the ATPase inactive OsRca mutants}

It has been reported, that as typical for AAA+ ATPases, a Walker A lysine residue and a Walker B aspartate residue of Rubisco activase in spinach and tobacco respectively, is crucial for ATP binding and hydrolysis [148, 163]. So we mutated lysine 115 and aspartate 173 to alanine (numbering as per rice Rca) at these critical sites in the ATPase active site (Fig. 3.11A). Site-directed mutagenesis was performed to produce mutants of OsRca $\alpha$ and OsRca $\beta$ as described in material and methods. The mutations at above positions led to a complete loss of ATPase activity in the mutant proteins as expected (Fig. 3.11B). This permitted a more reliable interpretation of the experimental data in terms of subunit doping.

Subunit mixing experiments were then performed with these mutants as described earlier in section 3.5. This time, a reduction in ATPase activity of the OsRca $\beta$ at different ratios was observed without any interfering activity of the OsRca $\alpha$ unlike previously. We observed a reduction of 55-70\% in ATPase activity (Fig. 3.11C) at an equimolar ratio ( $5 \mu \mathrm{M}$ mutant), while a decline of $75-85 \%$ was observed when the mutant/WT ratio was increased to $2: 1$ (10 $\mu \mathrm{M}$ mutant) in the final reaction mix. The previous studies with ATPase inactive mutants such as D231R and D174A in spinach have reported a reduction of $80 \%$ and $40 \%$ ATPase activity respectively for tobacco Rca at 4:1 mutant/WT ratio [163]. Interestingly, here we found that mixing of ATPase inactive Rca $\beta$ mutants reduced the activities of the wild-type (WT) Rca $\beta$ subunits to a similar extent as Rca ${ }^{2}$ mutants (Fig. 3.11C). This suggested that the C-terminal extension of Rca $\alpha$ was not involved in modulating the activity of the oligomer. 

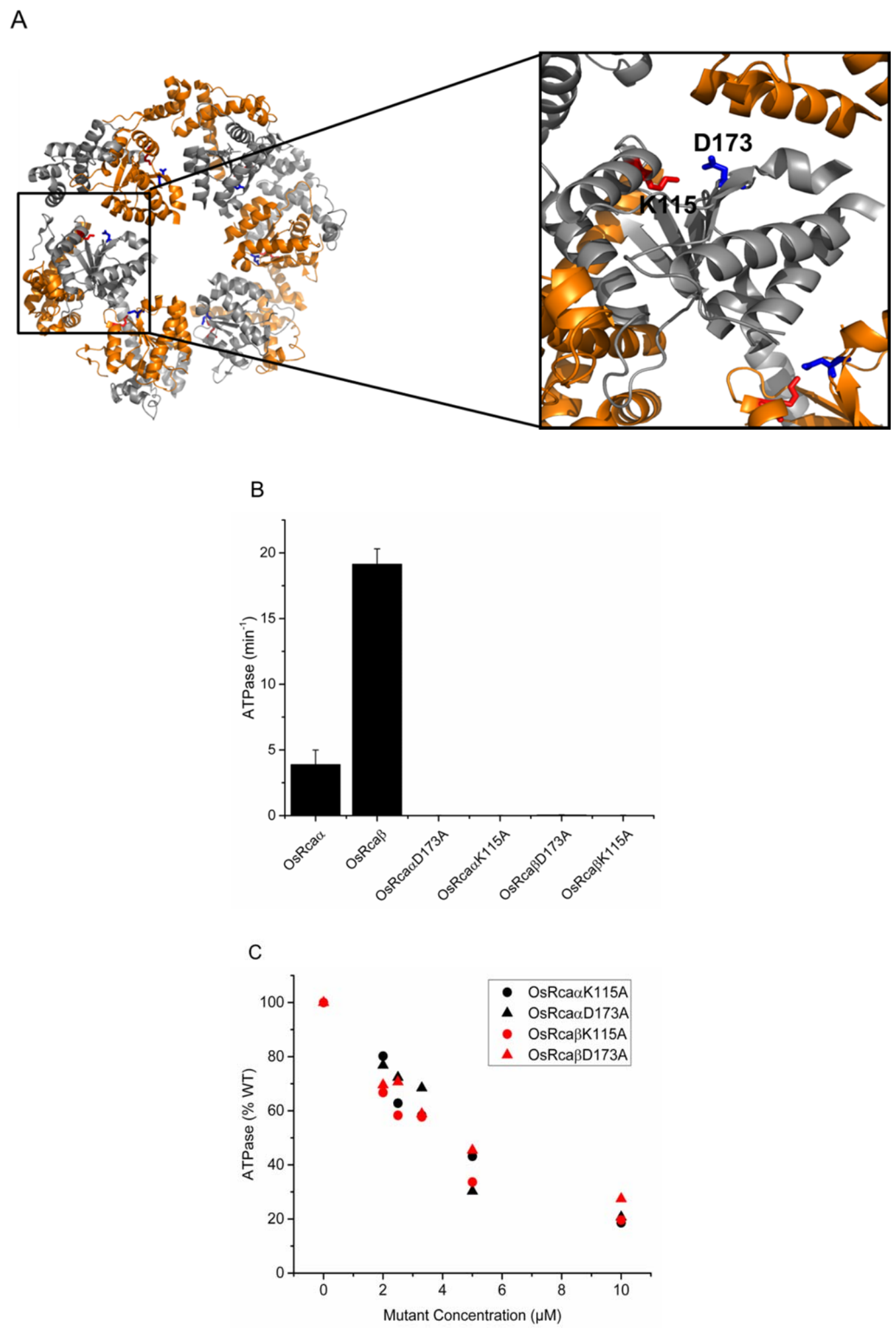

Fig. 3.11: Mutations at the Walker $A$ and Walker $B$ motifs of Rubisco activase abolish ATPase activity. (A) Ribbon structure of hexameric model of tobacco Rubisco activase (PDB id: 3ZW6) highlighting K115 and D173 residues targeted for mutagenesis in red and blue respectively. (B) ATPase assays were performed as usual $\left(5 \mu \mathrm{M}\right.$ protomer, $\left.25^{\circ} \mathrm{C}\right)$ with site directed mutants of Rca 
and Rca $\beta$ isoform responsible for ATPase activity. WT (wild-type) Rca $\alpha$ and Rca $\beta$ isoforms were used as positive control. (C) Effect of subunit mixing of ATPase inactive mutant of the $\alpha$ and the $\beta$ isoform on ATPase activity of the WT $\beta$ isoform. WT active OsRca $\beta$ was pre-incubated with the ATPase inactive mutants at 260-280 $\mu \mathrm{M}$ Rca protomer concentration range for $10 \mathrm{~min}$. ATPase assays were then performed at $25^{\circ} \mathrm{C}$ by keeping the final concentration of OsRca $\beta$ as constant $(5 \mu \mathrm{M})$ and titrating up the concentration of inactive mutant proteins at different proportion in the final reaction mix as indicated.

These results further validate that Rca subunits do not function independently but exhibit subunit cooperativity by rapidly exchanging subunits as reported previously for tobacco Rca [129, 163]. Thus, mechanistically Rca follows a concerted or subglobally cooperative mechanism of ATP hydrolysis where all or a specific minimal number of subunits are required to hydrolyse ATP simultaneously and oligomers containing less than the required number of active subunits become inactive [193, 194, 217]. These considerations are further discussed in detail in chapter 5. It also provides a mechanism to modulate the ATPase activity of the Rca $\beta$ subunits by Rca $\alpha$ isoform in vivo. By forming rapidly exchanging hetero-oligomeric mixtures of lowactivity Rca $\alpha$ and high-activity Rca $\beta$ isoforms, modulating the concentrations of Rca $\alpha$ allows the activity of the oligomer to be reduced with the concentration of the Rca .

\subsubsection{Further evidence for hetero-oligomerization between Rca isoforms}

Rubisco activases are polydisperse in nature and change their oligomeric states with concentration and in presence of nucleotides [133-135, 141]. This property of Rca was exploited to validate the physical interaction between Rca isoforms. Initially, we studied the elution behaviour of the rice activase isoforms using analytical gel filtration chromatography.

Both rice isoforms were eluted as polydisperse oligomers with a size corresponding to the apparent molecular weight of 75-440 kDa depending on their concentrations (Fig. 3.12A and B). Peaks corresponding to the larger oligomeric species were observed at higher concentration of activases and vice-versa. The $\alpha$ isoform eluted earlier than the $\beta$ isoform at low concentration (5 $\mu \mathrm{g}$ in $50 \mu \mathrm{l}, 0.1 \mathrm{mg} / \mathrm{ml}$ ). Therefore, this concentration of both Rca was chosen for another experimental run in the column to detect the shift in the peaks (Fig. 3.12 C). It was observed that when the same amount of $\alpha$ and $\beta$ isoform (5 $\mu \mathrm{g}$ each) were mixed and run together in the same buffer (see 
figure legend), the mixture was eluted as a single peak rather than two separate peaks of individual protein (Fig. 3.12C). The peak of the mixture was distinct as compared to the individual peak of each isoform alone (Fig. 3.12C). Moreover, the mixed sample of $10 \mu \mathrm{g}$ total proteins $(5+5)$ runs almost intermediately to $10 \mu \mathrm{g}$ of the individual protein (Fig. 3.12D), which clearly indicate the shift in the peak was due to the interaction between two isoform subunits and therefore proteins are behaving as a single entity in the mixture.

This was further validated by running the samples from eluted fractions obtained from the experiment in Fig. 3.12C on the SDS-PAGE (Fig. 3.12E). The SDS PAGE analysis of the earliest fractions of the mixed protein run indicates the presence of both isoform which runs as a single entity in mixture while the $\beta$ isoform was completely undetectable in the same fraction when eluted individually (Fig. 3.13E). This indicates changes in the elution pattern of the $\beta$ isoform when run together with the $\alpha$ isoform which is only possible when two subunits interact among each other.

A

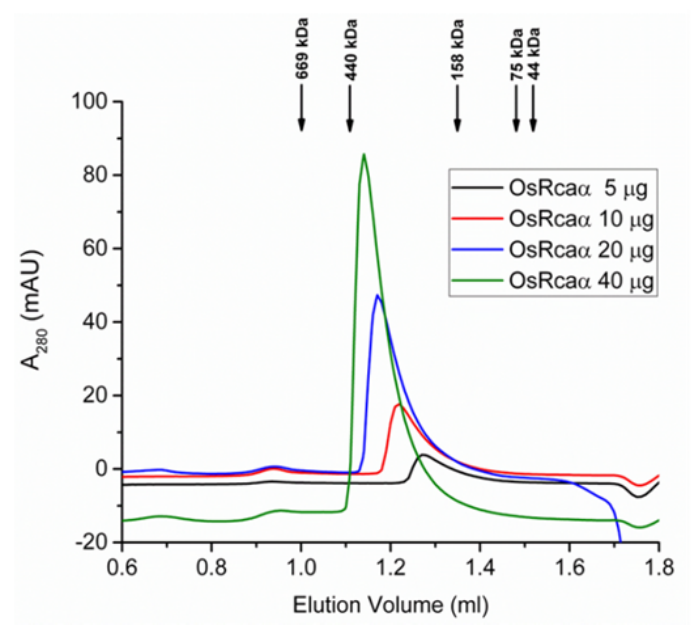

B

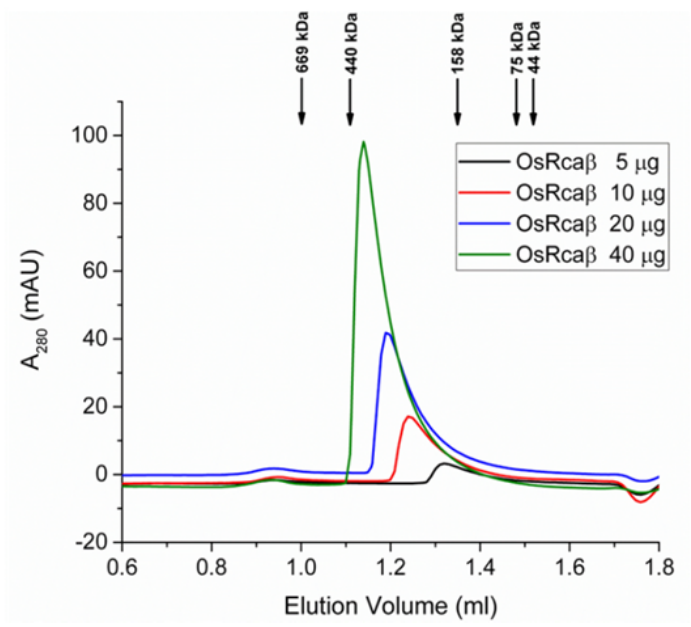



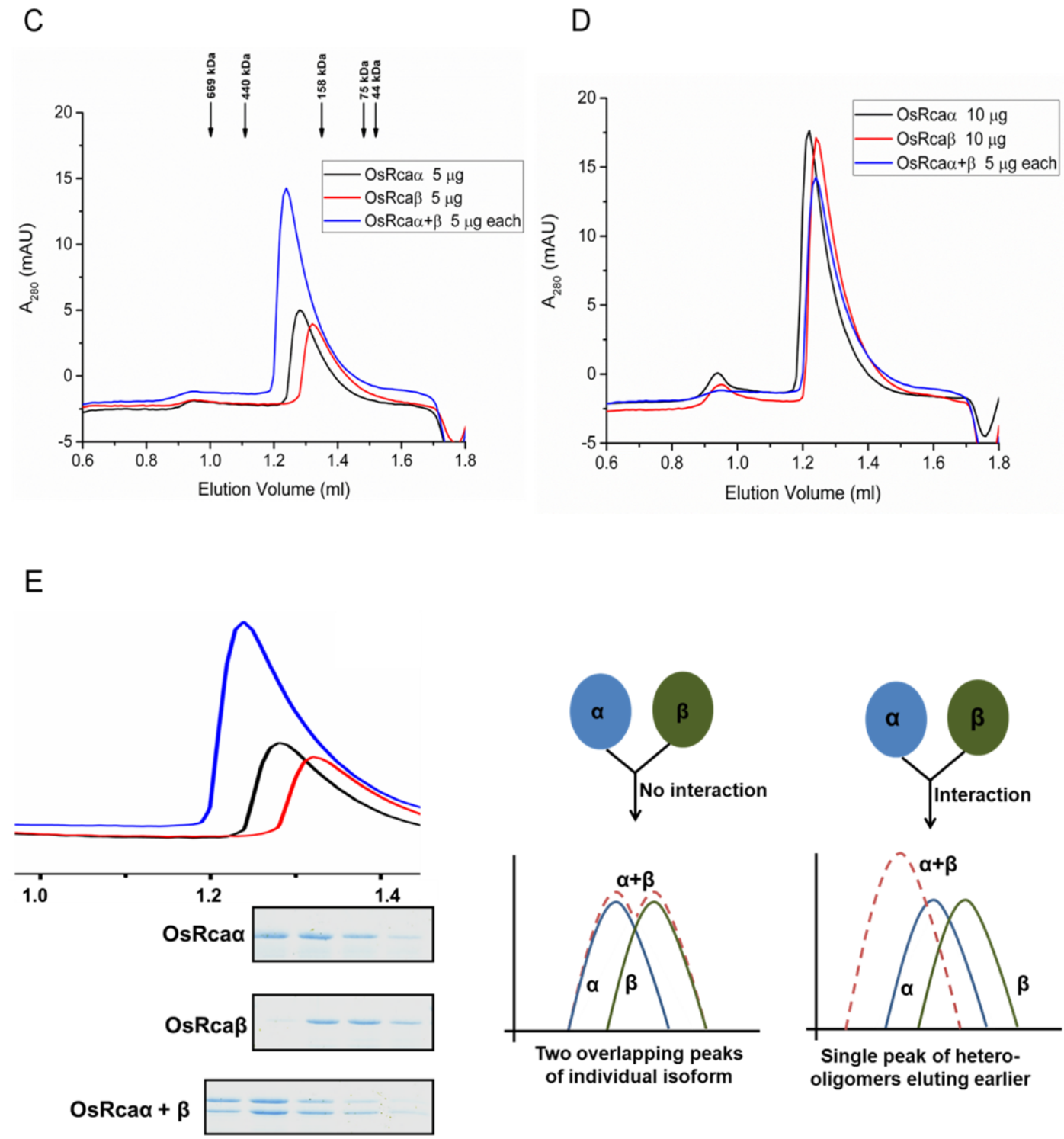

Fig. 3.12: Subunit interaction study using analytical gel filtration and SDS-PAGE: (A and B) Analytical gel filtration chromatography of OsRca $\alpha$ and $\beta$ at different concentration performed using a Superdex $2003.2 / 30$ increase column in buffer: $20 \mathrm{mM}$ Tris- $\mathrm{HCl} \mathrm{pH} \mathrm{8.0,50} \mathrm{mM} \mathrm{NaCl,} 5 \mathrm{mM} \mathrm{MgCl}$, $0.5 \mathrm{mM}$ ATP. (C and D) Analytical gel filtration chromatography of rice isoforms in mix $(\alpha+\beta)$ along with control elution peaks in same buffer as A and B. E. 12.5\% SDS-PAGE analysis of the samples from the fractions obtained from $\mathrm{C}$ (zoomed). Each fraction was $50 \mu \mathrm{l}$ in volume correspondingly. Bands have been positioned exactly below the fractions or respective elution volumes (see the magnified image) which were used for SDS- PAGE analysis as depicted (left). Schematic representation of the experiment and predicted outcomes differentiating the situation between interaction and non-interaction among two subunits (right). Scheme is only for illustrative purpose. 


\subsection{Subunit mixing and redox state does not affect the thermotolerance of the rice activase}

Considering the fact that Rca $\alpha$ has been reported to confer thermostability to Rca $\beta$ in spinach $[141,178]$ and observed to be the most upregulated protein under heat stress in the wild rice species Oryza meridionalis [197], we hypothesised that OsRca $\alpha$ may confer thermostability to rice Rubisco activase, possibly in the oxidized, low activity form. Hence, in vivo overexpression of the OsRca may represent a protective mechanism preventing denaturation of the activase in addition to downregulating $\mathrm{CO}_{2}$ fixation during heat stress. However, the redox state of the proteins in the earlier experiments was not reported [141, 178, 197]. So, we also hypothesized that the OsRca $\alpha$ may vary in its thermotolerance depending on whether the enzyme is reduced or oxidized.

We conducted subunit mixing experiments between the OsRca $\alpha$ and $\beta$ at an equimolar concentration at different temperatures (Fig. 3.13A). We also performed ATPase activity assay for OsRca $\alpha$ under reduced condition at different temperatures to test this hypothesis (Fig. 3.13). However, it was concluded that subunit mixing in rice did not further improve the thermal stability of rice Rubisco activase. Also, the change in redox state of Rca confirms the role of thioredoxin- $f$ in modulating the activity of Rca $\alpha$ isoform but did not improve the thermotolerance. 
A
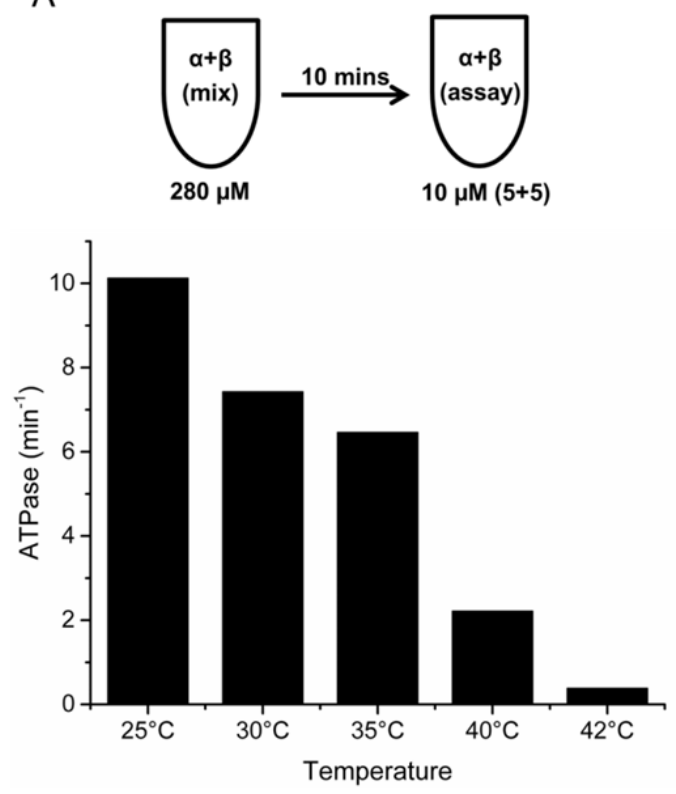

B
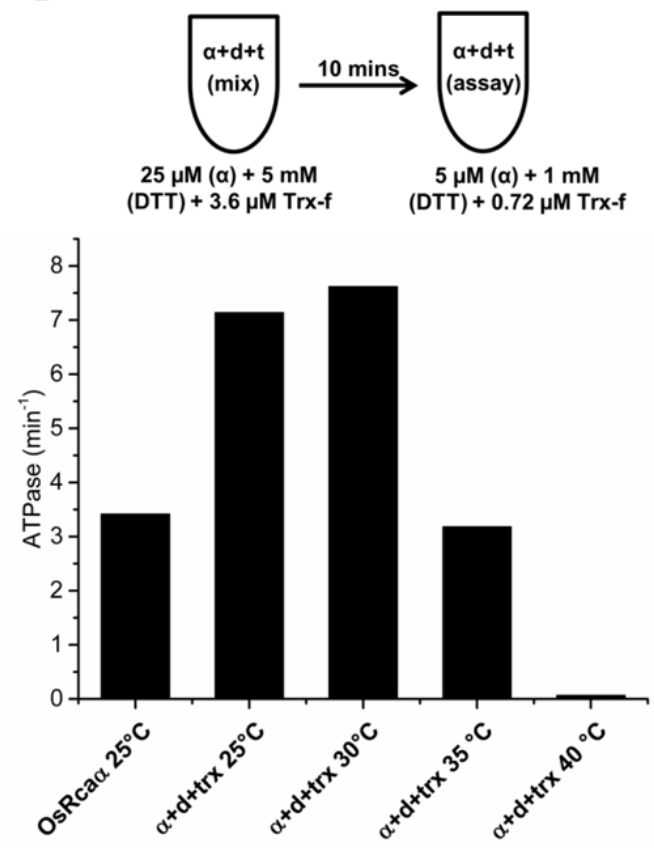

Fig. 3.13: Effect of subunit mixing and Thioredoxin-f on thermostability. (A) OsRca $\alpha$ and OsRca were mixed at around $280 \mu \mathrm{M}$ Rca protomer concentration and incubated at the indicated temperature for $10 \mathrm{~min}$. ATPase assays were then performed at $25^{\circ} \mathrm{C}$ at an equimolar concentration (1:1) of $5 \mu \mathrm{M}$

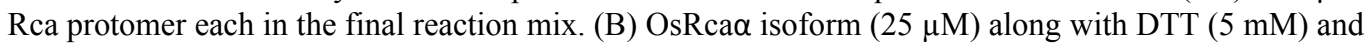
thioredoxin-f $(3.6 \mu \mathrm{M})$ were incubated separately and the mixture was heated at the indicated temperature for $10 \mathrm{~min}$. ATPase assays were then performed at $25^{\circ} \mathrm{C}$ with $5 \mu \mathrm{M}$ Rca protomer, $1 \mathrm{mM}$ DTT and $0.72 \mu \mathrm{M}$ thioredoxin-f in the final reaction mix. (OsRca $\alpha$ or $\alpha$ : $\alpha$ isoform of Rubisco activase, d: DTT, trx: thioredoxin-f)

In summary, we have presented a biochemical and biophysical characterization of rice activase isoforms in this chapter. The ability to rapidly assay defined subunit mixtures allows us to probe the function of the enzyme further. These ideas are further explored in chapter 5 . 


\section{Characterization of a thermostable Rubisco activase from Agave tequilana}


It has been demonstrated earlier and also in the present study (section 3.4) that Rubisco activase is a thermolabile enzyme. This property is believed to be directly responsible for the inhibition of photosynthesis at moderately high temperature $[10$, 11]. In contrast, higher plant Rubisco has been demonstrated to be thermostable even at elevated temperature, indicating that the loss of photosynthetic $\mathrm{CO}_{2}$ fixation is the result of thermal denaturation of Rca [10]. It has been predicted that the global temperature may rise by $2-5^{\circ} \mathrm{C}$ by the end of this century [218] and an estimation showed that each degree rise in temperature in an average growing season results in up to $17 \%$ decrease in yield [219]. Therefore it is necessary to take steps to minimise the effect of heat stress on the efficiency of photosynthesis for increasing crop yield and prepare the crops for the future climatic changes [18].

It has been reported that having a more thermostable Rca variants results in higher photosynthetic rate, improved developmental patterns, higher biomass and increased seed yields [12]. This study was conducted using gene shuffling to generate variants of the $\beta$ isoform of Arabidopsis thaliana followed by screening these mutants for more thermostable variants. Another study on the same plant species demonstrated that transforming the arabidopsis Rca mutant plants with a more thermostable chimeric activase between tobacco and arabidopsis containing the Rubisco recognition domain ( $\alpha$-helical subdomain) from arabidopsis showed a higher photosynthetic rate under moderate heat stress [13]. It has been predicted that introducing Rca from a warm season plants like cotton into a cool season species like Camelina would improve the thermotolerance of photosynthesis by as much as $5{ }^{\circ} \mathrm{C}$ $[15,220]$. Thus, thermal stability of the Rubisco activation process could be enhanced either by generating thermostable variants of Rubisco activase or by introducing or replacing the native Rubisco activase with more thermostable activase from other compatible organisms.

Plants from a high temperature environment may not require a particularly thermostable activase, since they often possess powerful leaf cooling mechanisms, especially transpiration $[175,221,222]$. We noted that this mechanism would not be available to CAM plants growing in a hot desert environment, where Calvin-Benson cycle function will be taking place in the light under high exposure of heat, with the stomata closed to minimize water loss [223]. Consequently we reasoned that, like 
other enzymes required during daytime, CAM activases should be relatively thermostable $[224,225]$. To test this hypothesis, we targeted an activase system from a CAM plant, Agave tequilana.

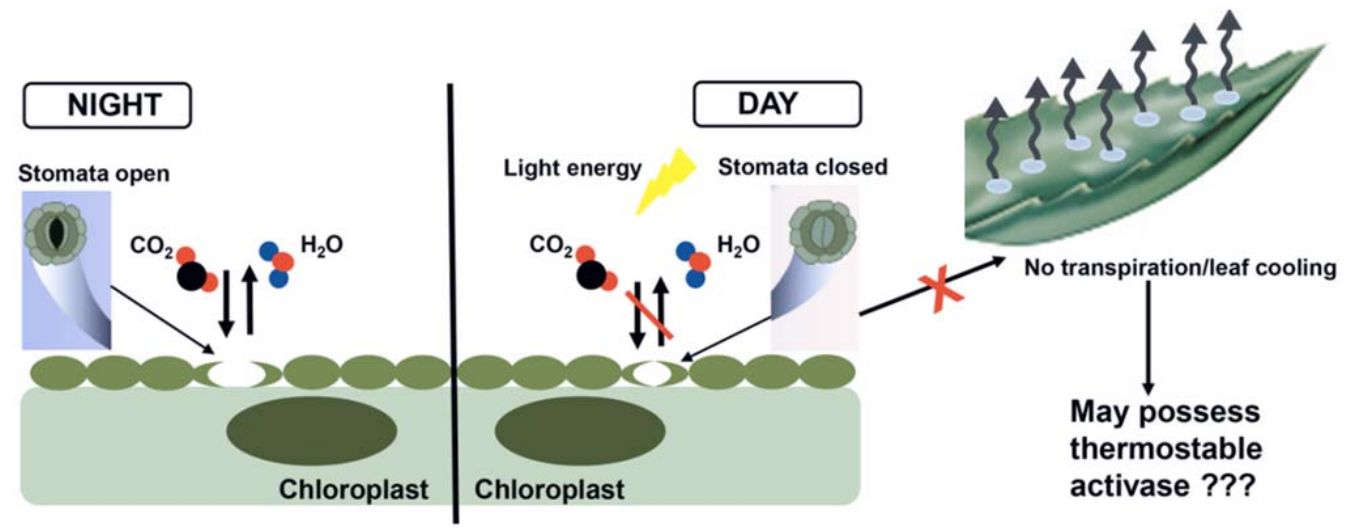

Fig. 4.1: Schematic diagram of a day night cycle in a typical CAM plant: A partial CAM pathway showing $\mathrm{CO}_{2}$ uptake and release of water through open stomata during night, while during day stomata remain closed to prevent the exit of internally released $\mathrm{CO}_{2}$ and water while performing photosynthesis.

In this chapter, we describe a biochemical characterization of a thermostable and highly effective activase system from agave in a comparative study with their rice homologues. This promising thermostable system was then utilized for engineering a more thermotolerant and efficient variant of rice activase and locating the regions and key residues responsible for such properties. This was attained by generating chimeric mutants between rice and agave by inter-swapping their major domains supplemented with site directed mutagenesis. Thus we have attempted to explore the natural diversity of activase proteins from different environments to mechanistically understand their biophysical and biochemical properties. This approach should assist in overcoming the limitations of existing activases like slow activation rate and thermolability.

\subsection{Purification of Agave tequilana Rca isoforms}

The genes encoding $\alpha$ and $\beta$ isoform of Rubisco activase of Agave tequilana (AtRca $\alpha$ and AtRca $\beta$ respectively) were identified from the transcriptome assemblies of $A$. tequilana [182]. Unlike rice, two Rca isoforms in agave are not the product of alternate splicing as the genes encoding them were found to be present on the different locus in the transcriptome data. Consequently, a few residues among the 
main part are different in two isoform (Fig. 4.2). The $\alpha$-isoform of agave possesses around 40 additional residues as a C-terminal extension. Aligning the amino acid sequences revealed that both isoforms of $A$. tequilana shows $\sim 86 \%$ sequence identity with the rice activases (Fig. 4.2).
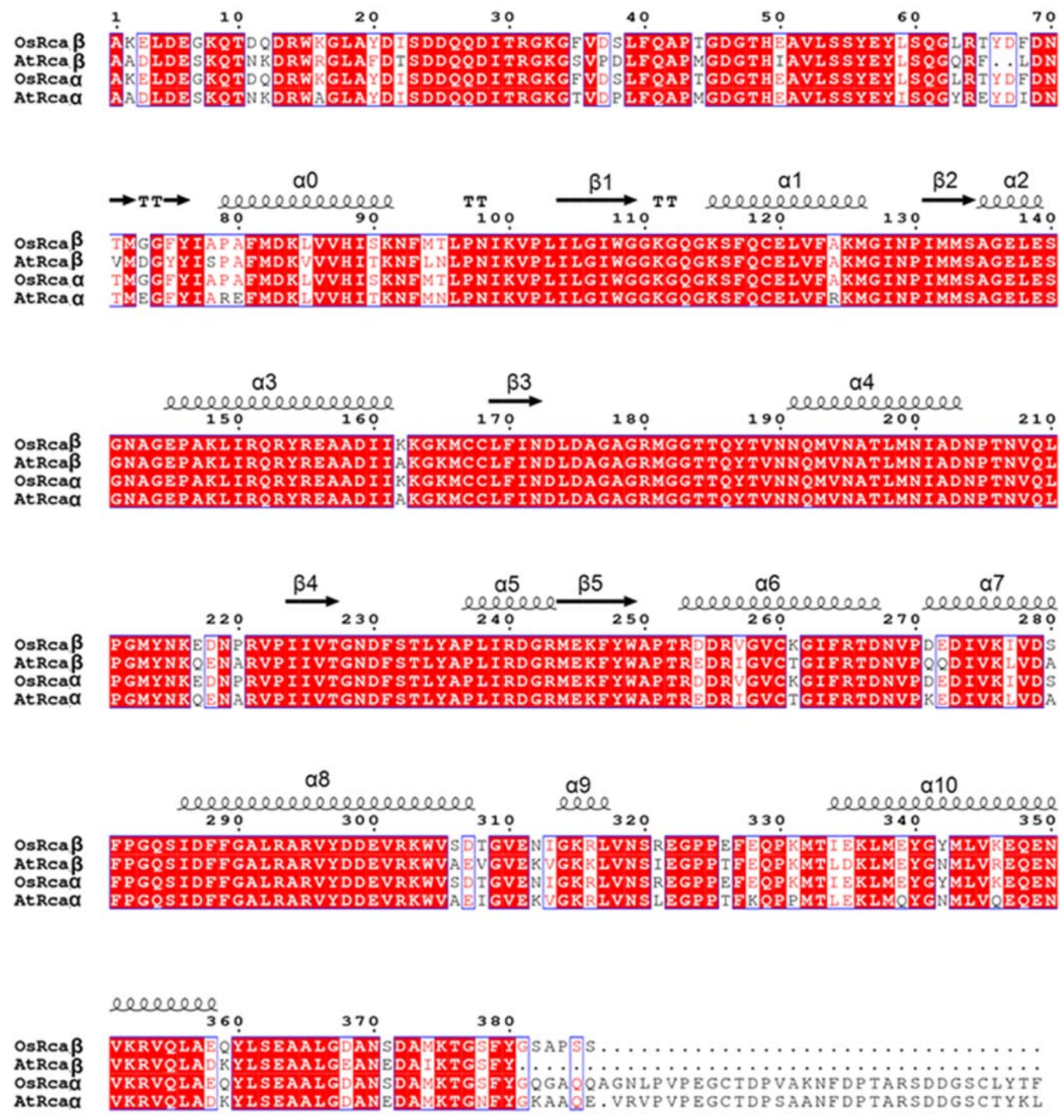

Fig. 4.2: Seqeunce alignment of Oryza sativa and Agave tequilana activase isoforms. Amino acid sequences of two Rubisco activase (Rca) isoforms of Oryza sativa and Agave tequilana were aligned using ESPript [184]. Rice and agave has two Rca isoforms $\alpha$ and $\beta$. The two isoform in rice (accession code: P93431-1 and P93431-2) are the product of alternate splicing only differing in the C-terminal sequence as shown. OsRca $\alpha$ has 33 extra amino acids at the C-terminal and also differ in 5 amino acid preceding to this extension. The two isoform in agave are the product of different gene (locus 3705 , and 27298) [182]. AtRca $\alpha$ also possesses around 40 extra residues as a C-terminal extension. 
Following gene synthesis, A. tequilana activases were heterologously expressed in E.coli BL21 as a N-terminal fusion with hexa-histidine tagged ubiquitin ( $\left.\mathrm{His}_{6} \mathrm{Ub}\right)$ and purified exactly as described for rice Rca. The average yield of protein from 11 culture of E.coli BL21 grown in LB media after induction with IPTG at $23{ }^{\circ} \mathrm{C}$ was found to be $\sim 12-15 \mathrm{mg}$ of pure protein.

A

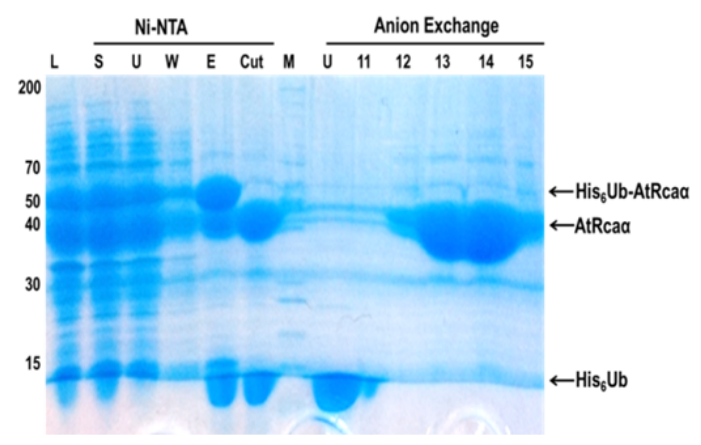

C

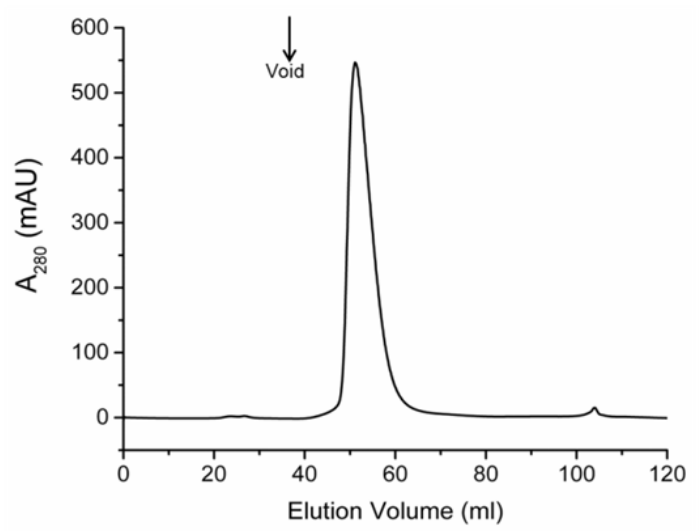

B

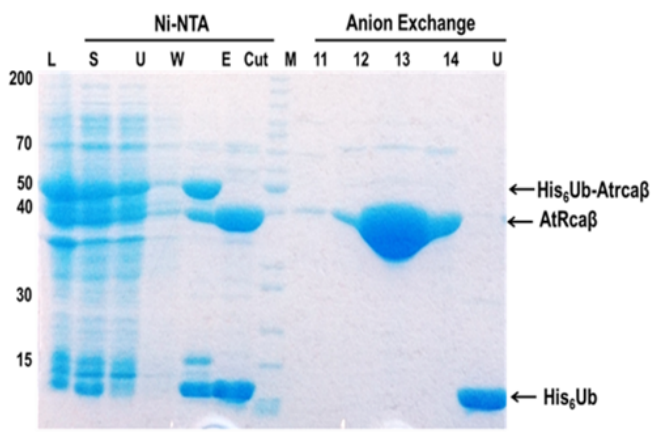

$\mathrm{D}$

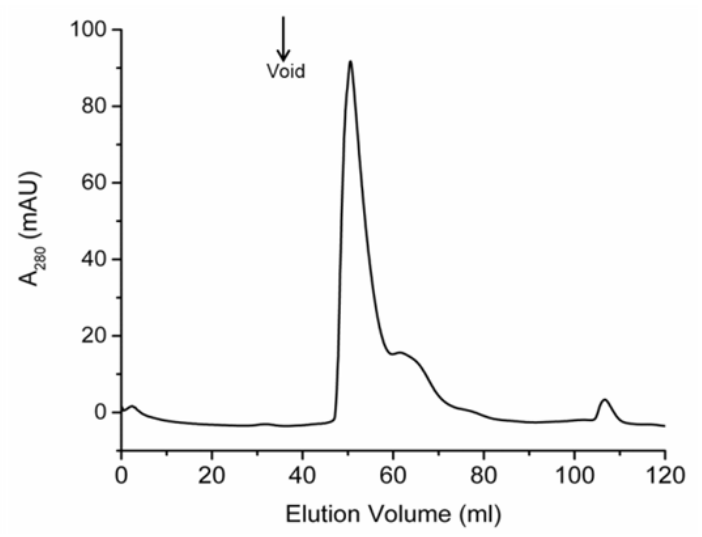




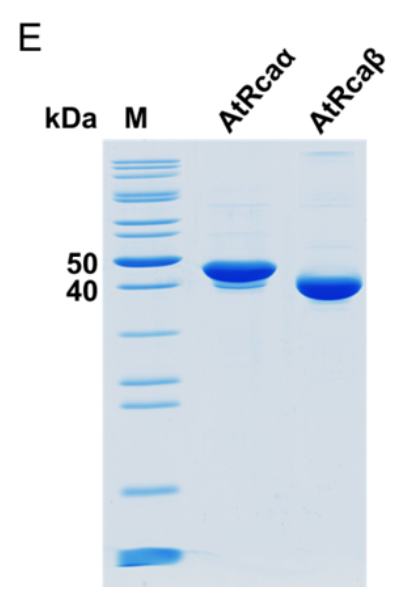

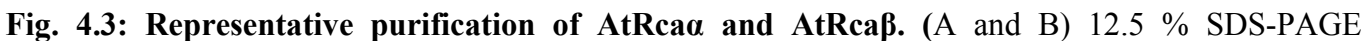
analysis. Successive purification by IMAC (Ni-NTA), followed by Usp2 mediated cleavage of Nterminal tag and anion exchange chromatography (AIEC) yielded pure protein.(M: Molecular Weight Marker, L:Lysate, S:Soluble, Ni-NTA: Fractions after IMAC, U: Unbound, W:Wash,: E: Final eluate, Cut : eluate after USP2 mediated cleavage of Ubiquitin. The numbers indicates the SDS sample from respective fraction of AIEC). (C and D) Size exclusion chromatograms of AtRca $\alpha$ and AtRca $\beta$ using a superdex 200 16/600 column, showing a single peak of each Rca. (E) Final purified protein on 16\% SDS-PAGE gel.

\subsection{Both agave isoforms were biochemically active and can activate inhibited rice Rubisco more efficiently than rice Rca}

Once the proteins of interest were in hand, first of all, spectrophotometric Rubisco activation assay was performed between RuBP inhibited rice Rubisco (ER) with agave activases to examine if rice Rubisco could be activated by agave activase. Generally plant activases tend to be broadly compatible, and incompatibility has only been demonstrated between activases and Rubiscos derived from Solanaceae and nonSolanaceae species [226]. Consistently, it was found that agave activases can activate rice Rubisco (Fig. 4.4A). The average activation rates for AtRca $\alpha$ and $\beta$ were around 42 and $36 \mathrm{nM} \mathrm{ECM} \min ^{-1}$ respectively compared to $\sim 2.7$ and 7-8 $\mathrm{nM} \mathrm{ECM} \min ^{-1}$ for OsRca $\alpha$ and $\beta$ respectively (Fig. 4.4C). It was surprising to us that their ability to activate rice Rubisco was 4-5 fold higher than the rice activase isoform (OsRca $\beta$ ) under the chosen assay conditions. Similarly, both isoforms of AtRca displayed a relatively high ATPase activity $\sim 32 \mathrm{~min}^{-1}$ (Fig. 4.4C) unlike OsRca, where the $\beta$ isoform showed a much higher activity $\left(\sim 22-25 \mathrm{~min}^{-1}\right)$ than the $\alpha$ isoform $\left(\sim 6-8 \mathrm{~min}^{-}\right.$ ${ }^{1)}$. 
Based on the above results one possiblity is that agave activases are more efficient due to their inherent high activity rather than enhanced Rubisco-Rca interaction. Alternatively, it could be postulated that agave activases possess higher affinity for the rice Rubisco than the cognate rice activases. To distinguish between these two possibilities, we incorporated polyethylene glycol (PEG 3350) in the Rubisco activation assay (Fig. 4.4B).

PEG is a crowding or solvent excluding reagent which has been described thermodynamically to promote self-association and activity coefficient of proteins [227]. Macromoleculer crowding approach using PEG 3350 has been previously demonstrated to stimulate the activase activity for plant Rca and the recently discovered CbbQO-type activase of chemoautotrophic bacteria [115, 136]. Moreover, PEG promotes self-association of Rca which not only increased the specific activity of the activase but also enhances the affinity for ATP and interaction of Rca and Rubisco [136]. The concentration of Rubisco ( $\sim 200 \mathrm{mg} / \mathrm{ml})$ and Rca $(\sim 7-20 \mathrm{mg} / \mathrm{ml})$ in chloroplast stroma is extremely high $[8,107]$, compared to in vitro assay conditions. Therefore, it has been speculated that this crowding environment allows Rubisco and activase to be in close proximity and facilitates them to function efficiently. Thus under in vitro assay conditions, crowding agents assist in simulating the in vivo environment causing higher interaction between Rca and Rubisco, ultimately leading to enhanced activation.

Consistently, we observed that activase activity of AtRca $\beta$ and OsRca $\beta$ was considerably enhanced by inclusion of 5\% v/v PEG 3350 in the assay (Fig. 4.4B). The activase activity of the AtRca $\beta$ increased by $\sim 2.8$ fold, while a remarkable $\sim 7$ fold stimulation was observed for OsRca $\beta$. This indicates that OsRca $\beta$ has a poor affinity for Rubisco under assay conditions, leading to slow activation which was overcome by the presence of PEG due to the crowding effect. AtRca $\beta$ on the other hand, already had a higher affinity for rice Rubisco and therefore did not benefit from the macromolecular crowding as much as cognate rice Rca. However, AtRca $\beta$ still maintained a 1.2 fold higher activation rate than OsRca $\beta$. Presence of PEG had no significant effect on the Rubisco activity of activated (ECM) and inhibited Rubisco (ER) complexes (Fig.4.4B) indicating that the stimulation was the outcome of enhanced protein-protein interaction between Rca and Rubisco. 

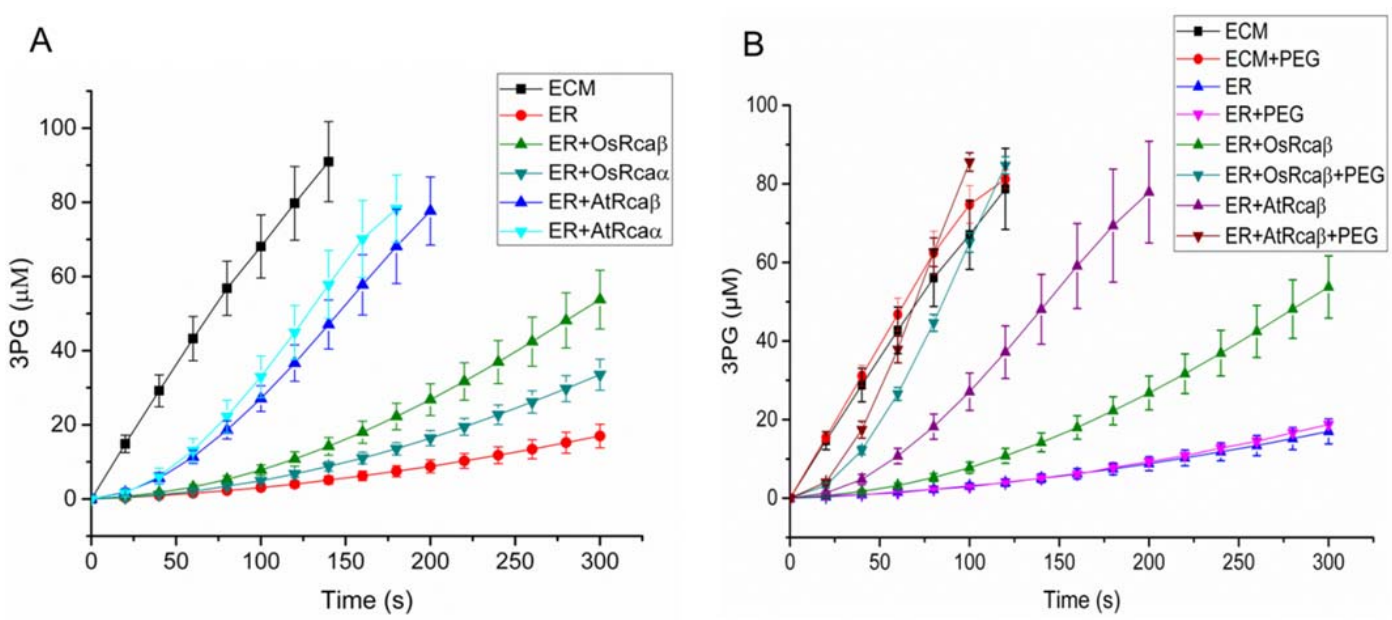

C

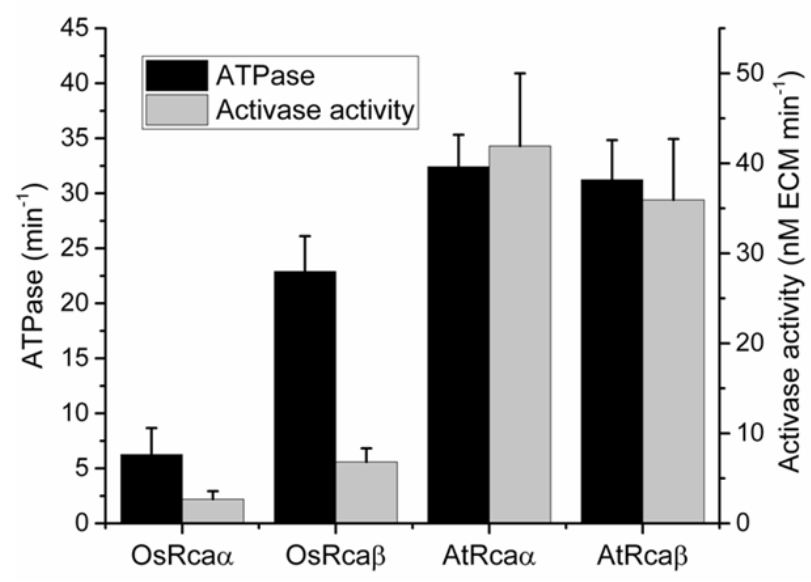

Fig. 4.4: Biochemical properties of Rubisco activase isoforms from Oryza sativa and Agave tequilana. (A) Rubisco activation assay of RuBP-inhibited rice Rubisco (ER) performed at $25^{\circ} \mathrm{C}(0.5$ $\mu \mathrm{M}$ Rubisco active sites, $5 \mu \mathrm{M}$ activase protomer) (B) Effect of crowding agent polyethylene glycol (PEG) 3350 on activase activity of the OsRca $\beta$ and AtRca $\beta$. Rubisco activation assays were performed as in A with inclusion of 5\% v/v PEG 3350 as indicated in the figure legends. (C) ATPase activity assays $\left(5 \mu \mathrm{M}\right.$ protomer, $\left.25^{\circ} \mathrm{C}\right)$ and quantified Rubisco activase activities of different Rca isoforms. Error bars indicate the standard deviations of mean values of three or more independent experiments.

\subsection{Agave Rca isoforms are highly thermostable}

Having established biochemical compatibility, thermal characterization of agave activases were performed in comparison to rice. The ATPase activity of the Rca $\alpha$ and $\mathrm{Rca} \beta$ of rice and agave after heat treatment at various temperatures is shown below (Fig. 4.5A) 
It was observed that while both the isoforms of rice were almost completely inactivated at $42{ }^{\circ} \mathrm{C}$, both agave isoforms still maintained their activity at $45{ }^{\circ} \mathrm{C}$. At higher temperatures, both isoforms start losing their activities. AtRca $\beta$ maintained around $60 \%$ of its activity at $50{ }^{\circ} \mathrm{C}$ and more than $40 \%$ of its activity at $52{ }^{\circ} \mathrm{C}$ before getting completely inactivated at $55{ }^{\circ} \mathrm{C}$. AtRca lost around $70 \%$ of its maximum activity at $47{ }^{\circ} \mathrm{C}$, and was completely inactivated at $50{ }^{\circ} \mathrm{C}$. Thus, the agave isoforms represented a highly thermostable Rubisco activase system for plants where each isoform of activase displayed $\sim 5-8{ }^{\circ} \mathrm{C}$ higher thermostablity as compared to their rice homologues in terms of their in vitro activities.

A

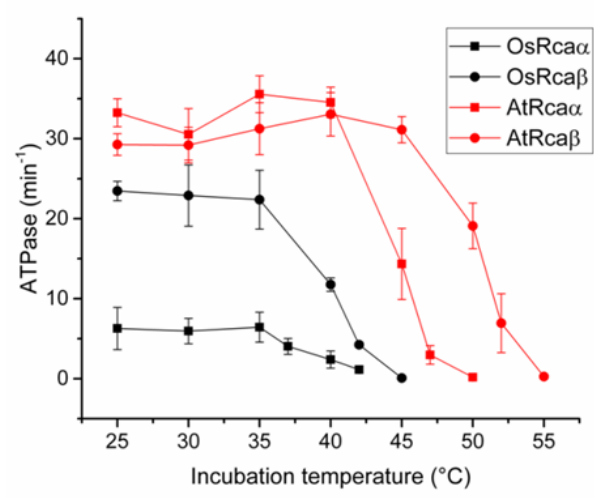

C

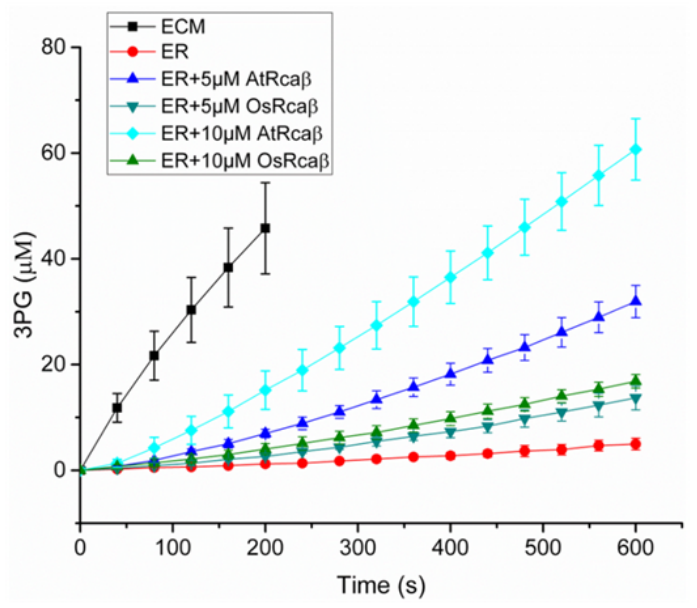

B

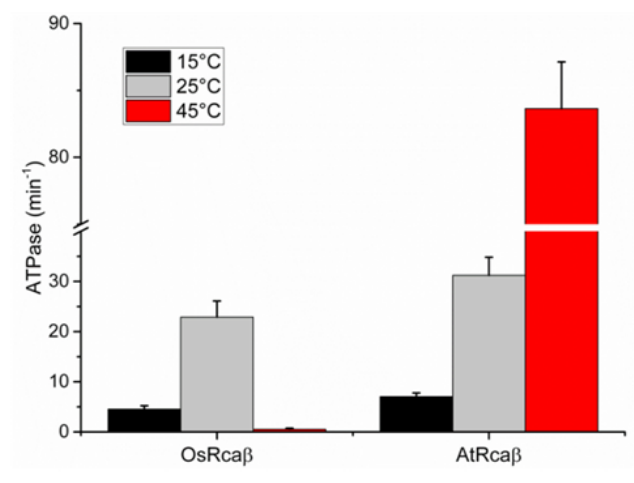

D

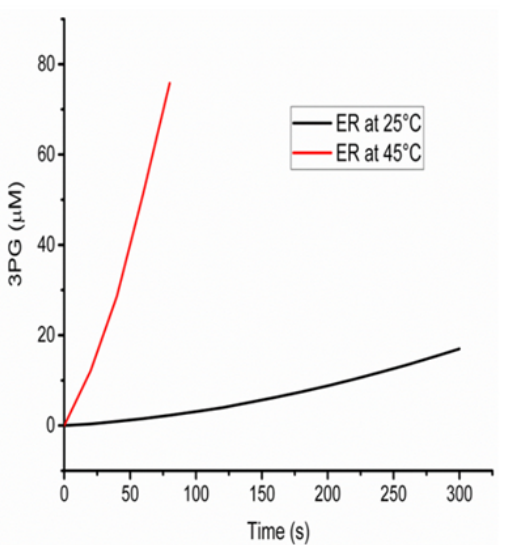

Fig. 4.5: Thermal response of Rca. (A) Comparative thermostability of activase isoforms in terms of ATPase activities. Activase isoforms (200-280 $\mu \mathrm{m}$ concentration range) were incubated at indicated temperature for $10 \mathrm{~min}$ and then ATPase activity was assayed at $25^{\circ} \mathrm{C}$ with $5 \mu \mathrm{M}$ Rca protomer in final reaction mix. (B) Comparative effect at low temperature. ATPase activity assay was performed at indicated temperature for OsRca $\beta$ and AtRca $\beta$. (C) AtRca $\beta$ has higher Rubisco activase activity than OsRca $\beta$ even at low temperature. Rubisco activation assays were performed at $15{ }^{\circ} \mathrm{C}$. (D) Rubisco activation assays could not be measured at $45^{\circ} \mathrm{C}$ due to instability of ER complex. Error bars indicate the standard deviations of mean values of three or more independent experiments. 
From the above data, it was quite evident that the agave activases are more potent at high temperature than the rice activases. However, it is commonly believed that increased thermostability is likely to trade-off with functionality at lower temperature [228] as previously observed for activases from different species [15, 229]. To test whether this was the case for the agave activases, we analyzed ATPase and activase activity of agave and rice Rca $\beta$ at a reduced temperature (Fig. 4.5B and C). It was observed that ATPase activity of AtRca $\beta$ and OsRca $\beta$ were drastically reduced at 15 ${ }^{\circ} \mathrm{C}$ (around 80\%) as expected from physical principles but quantitatively AtRca 3 still maintained higher ATPase rate (7-8 $\left.\mathrm{min}^{-1}\right)$ than OsRca $\left(4-5 \mathrm{~min}^{-1}\right)$ (Fig. 4.5B). At 45 ${ }^{\circ} \mathrm{C}$, OsRca $\beta$ became completely ATPase inactive, while a much higher ATPase activity $\left(\sim 85 \mathrm{~min}^{-1}\right)$ was observed for AtRca $\beta$.

Similarly, when the Rubisco activation assays were performed at $15{ }^{\circ} \mathrm{C}$, the activase activity of the OsRca $\beta$ was severely reduced and did not significantly improve with the concentration (Fig. 4.5C). While AtRca $\beta$ could still perform faster activation than OsRca $\beta$ even at $5 \mu \mathrm{M}$ concentration and full activation was achieved by increasing the concentration. It was clear from the above data that despite being thermostable AtRca $\beta$ proclaims high activity at either low or high temperature. However, Rubisco activation activity of Rca could not be measured at $45^{\circ} \mathrm{C}$ due to instability of rice ER complex at that temperature (Fig. 4.5D) as reported previously for tobacco ER [99]. Thus, AtRca $\beta$ exhibits no compromise in terms of their Rubisco activase activities as well as ATPase activity at low temperature compared to rice and displays superior functional characteristics at the temperatures tested.

\subsection{Rice and agave activase subunits form hetero-oligomers displaying intermediate functionality but subunit-specific thermostability}

Earlier studies of introducing thermostable activase into arabidopsis were performed using plants where the endogenous Rca had been deleted $[12,13]$. In rice such a line is not available. It would be more convenient to express thermostable activase in rice plants containing the endogenous activase. In earlier work, Rubisco activases from maize have been overexpressed in rice containing endogenous activases [230]. However, if the thermostable agave activases were to be engineered into rice plant in 
future, it is necessary to examine if activases from the two species interact with each other. And if so, it would be further important to ensure that an interaction between AtRca and OsRca should not result in loss of biochemical function due to the formation of 'inter-species' hetero-oligomeric complexes.

Therefore, first we characterized the biochemical properties of OsRca $\beta$ and AtRca $\beta$ mixture at an equimolar ratio (1:1). When the functional wild-type rice and agave Rca subunits were mixed and assayed, no reduction in functionality (both ATPase and Rubisco activase) was observed. Rather an intermediate level of activities were measured (Fig. 4.6A and B), suggesting the mixed species oligomers are not functionally impaired. However, it was difficult to distinguish between two distinct populations of homo-oligomers and the formation of functional hetero-oligomers. Therefore, subunit mixing experiments were performed between agave activase isoforms (AtRca $\alpha$ or AtRca $\beta$ ) and ATPase inactive Walker B mutant of rice (OsRcaßD173A). As shown previously for rice activase isoforms (section 3.5.1), a strong reduction in the ATPase activity of agave activase isoforms were observed (Fig 4.6C) when the concentration OsRcaßD173A was titrated keeping concentration of AtRca $\alpha$ or AtRca $\beta$ as constant. Taken together these results provide evidence for the formation of inter-species functional hetero-oligomers.

A

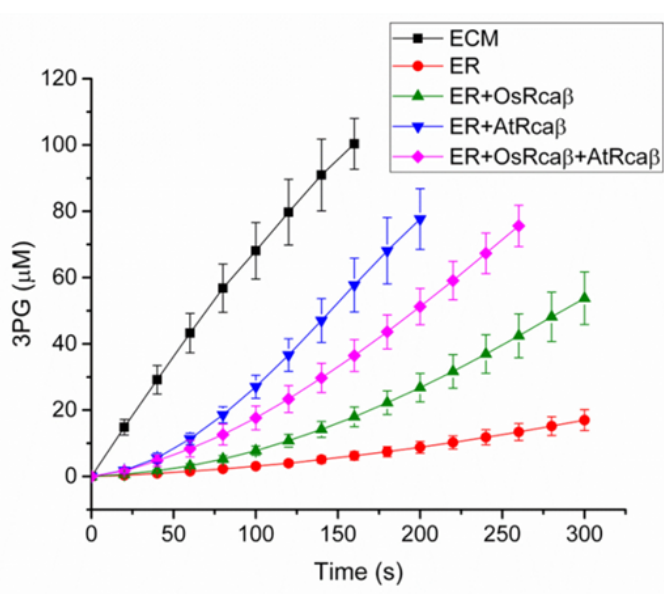

B

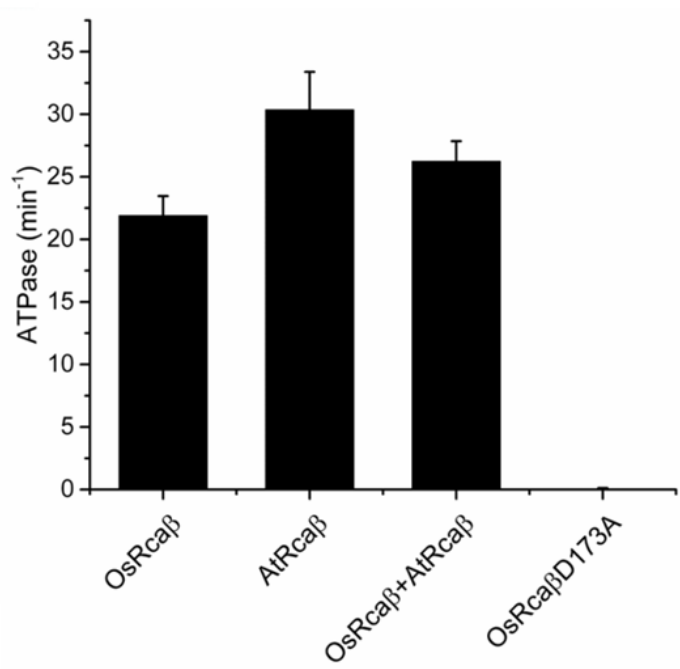



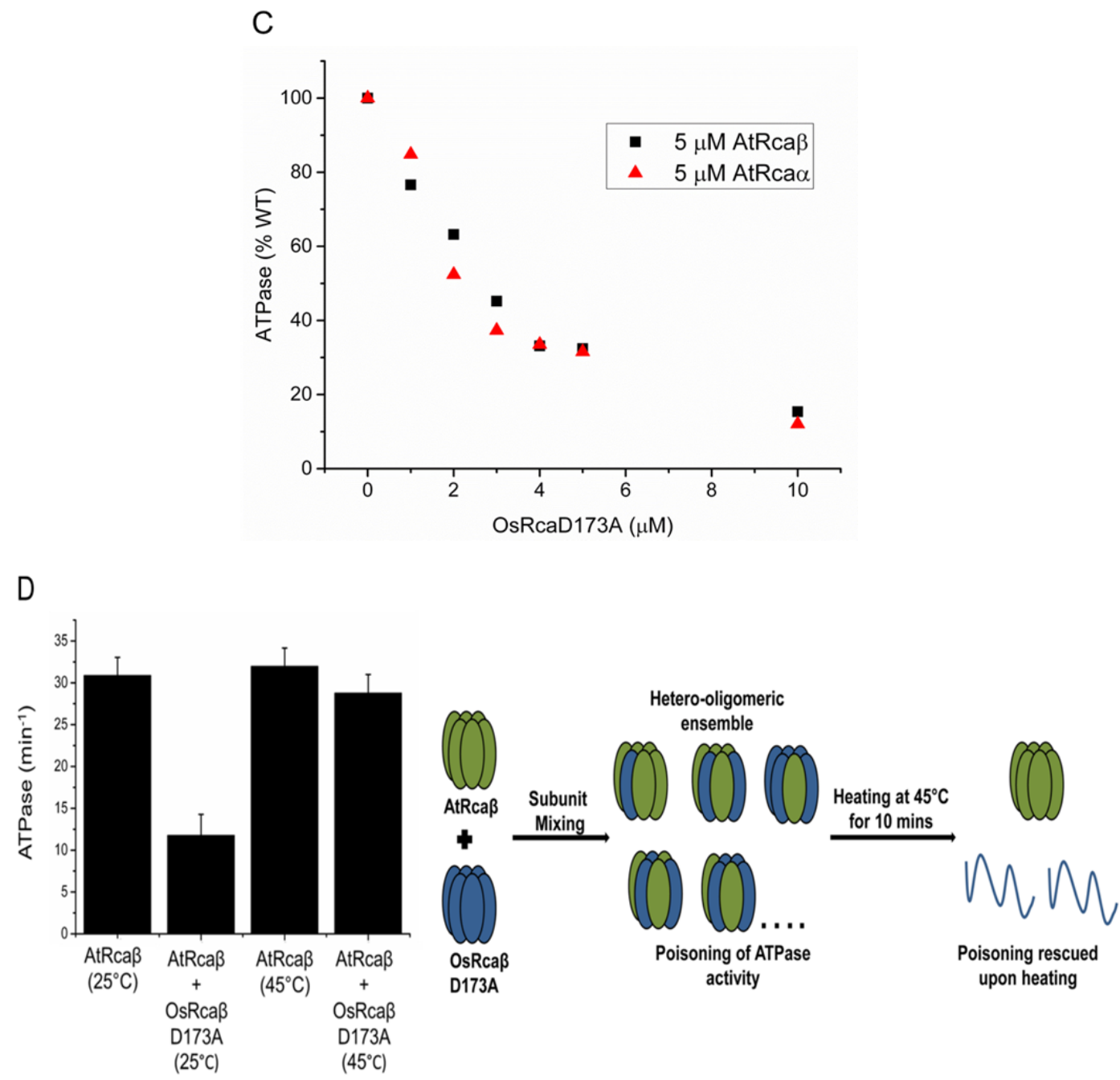

Fig. 4.6: Inter-species subunit interaction between the agave and the rice activase. Rubisco activation assay (A) and ATP assay (B) performed were performed at $25{ }^{\circ} \mathrm{C}$ using $5 \mu \mathrm{M}$ activase protomer $(2.5 \mu \mathrm{M}+2.5 \mu \mathrm{M}$ for AtRca $\beta+$ OsRca $\beta)$. ATPase is given per active site. (C) Evidence for hetero-oligomerization. AtRca was mixed with increasing amounts of ATPase inactive OsRcaßD173A for 10 minutes $(210-270 \mu \mathrm{M}$ concentration range). ATPase assay was then performed by maintaining the concentration of AtRca as constant $(5 \mu \mathrm{M})$ and varying the concentration of OsRcaßD173A in the final reaction mix. (D) Incubation at $45{ }^{\circ} \mathrm{C}$ restores the ATPase activity of impaired hetero-oligmers to the levels of AtRca $\beta$ wild-type. In (C) and (D) ATPase activity was calculated as turnovers per wildtype active site.

From the above results it was established that Rca functionality was maintained in terms of its activities. We then decided to ask whether hetero-oligomerization would affect the thermostability of the agave subunits. Therefore, we conducted an experiment where a 1:1 mixture of AtRca $\beta$ and OsRcaßD173A was heated together at $45{ }^{\circ} \mathrm{C}$ for $10 \mathrm{~min}$ before assay as depicted (Fig. 4.6D). It was observed that the 
subunit poisoning effect of OsRcaßD173A was rescued and AtRca $\beta$ almost regained its full original activity (Fig. 4.6D). This suggested that upon heating AtRca subunits remained in solution, while OsRca formed inactive higher order aggregates. Following the outcome from this experiment we predict that agave activases could perform their function independently at high temperature if expressed in vivo without adversely being affected by the lower thermostability of the rice isoforms.

\subsection{Characterization of chimeric mutants localized thermostability and high activase activity to the nucleotide binding domain (NBD) of AtRca $\beta$}

From the previous results it was clear that agave activases are highly thermostable as compared to their rice counterparts, in spite of high sequence identity ( $>86 \%)$ (Fig. 4.2). This prompted us to attempt to map the determinants of thermostability. Therefore, first chimeric mutants of Rca were generated by interchanging the nucleotide binding domain (NBD) and $\alpha$-helical $\mathrm{C}$-terminal sub-domain between rice and agave Rca (Fig. 4.7A).

A
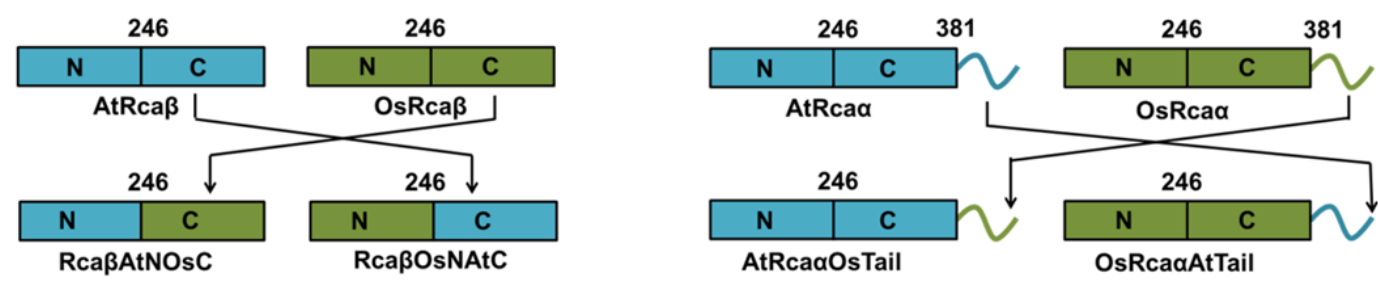

B

C
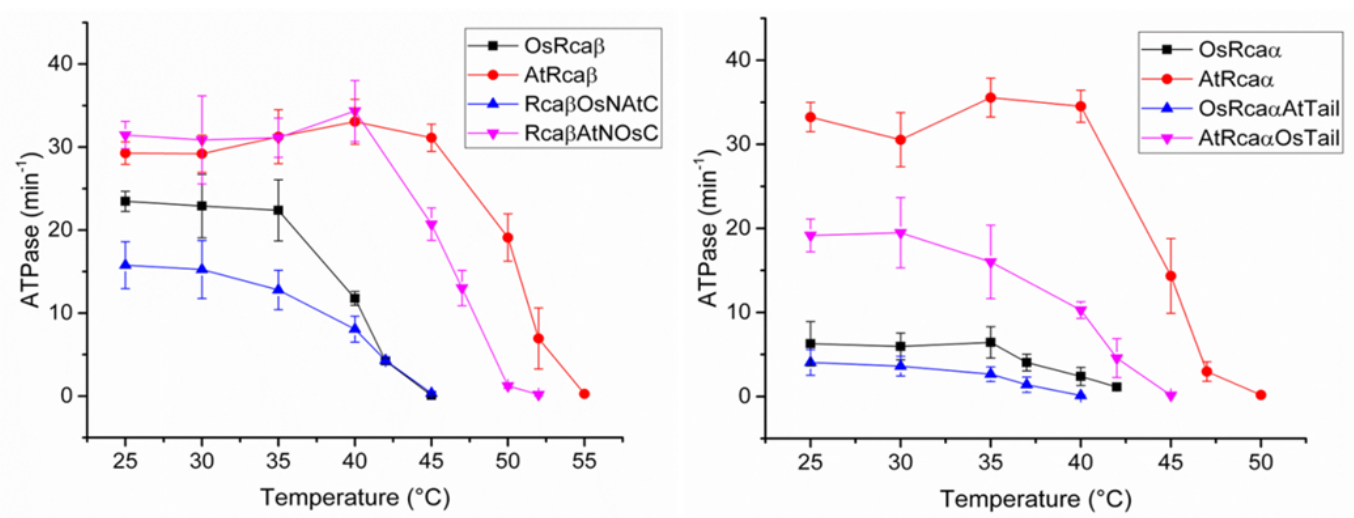


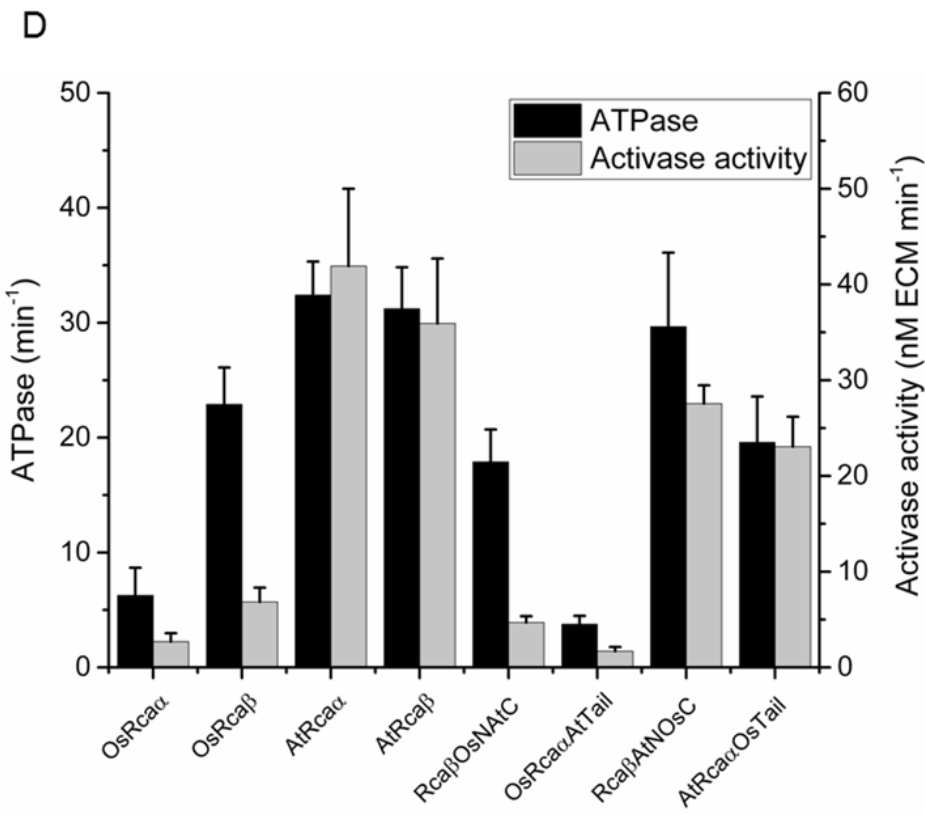

Fig. 4.7: Thermal and biochemical characterization of chimeric mutants. (A) Schematic diagram for the construction of chimeric mutants. (B \& C) Thermostability testing of chimeric mutants in terms of their ATPase activity compared to their wild type counterparts. ATPase assay was performed at 25 ${ }^{\circ} \mathrm{C}$ after incubating the proteins $(160-280 \mu \mathrm{M})$ at indicated temperature for $10 \mathrm{~min}$ such that the final concentration of the protein remains $5 \mu \mathrm{M}$ Rca protomer in the reaction mix. (D) ATPase and Rubisco activase activity of the chimeric mutants along with WT activases. Assayed at $25{ }^{\circ} \mathrm{C}, 5 \mu \mathrm{M}$ Rca protomer, $0.5 \mu \mathrm{M}$ ECM and ER. Error bars indicate the standard deviations of mean values of three or more experiments.

As observed for other activase homologues in previous studies [13, 154, 231], chimeric activases could be solubly expressed and purified in fully functional form (Fig. 4.7D). The thermal characterization of chimeric mutants showed that replacing the NBD of OsRca $\beta$ with the NBD of AtRca $\beta$ (RcaßAtNOsC) improves thermostability by $5-8{ }^{\circ} \mathrm{C}$ as compared to OsRca (Fig. 4.7B). This indicates that the NBD of agave can confer thermostability to rice activase. However, the improvement in thermostability of agave activase is not same as WT $\beta$-isoform of agave, and RcaßAtNOsC was observed to lose its functionality at a lower temperature (by $3{ }^{\circ} \mathrm{C}$ ) as compared to AtRca $\beta$. On the other hand, when the C-terminal domain of OsRca $\beta$ was replaced with the C-terminal domain of AtRcaß (RcaßOsNAtC), no improvement in thermostability was observed and the resultant chimeric mutants have the same thermostability as OsRcaß (Fig. 4.7B).

We were also interested to test whether the C-terminal extension of the AtRca $\alpha$ can confer thermostability to OsRca $\alpha$. Swapping the C-tail of AtRca $\alpha$ and OsRca $\alpha$ 
generated two chimeric activase mutants labelled as AtRca $\alpha$ OsTail and OsRca $\alpha$ AtTail (Fig. 4.7A). When the C-tail of OsRca $\alpha$ was transferred to AtRca $\alpha$ (AtRca $\alpha$ OsTail), the resulting chimeric mutant suffered from reduced thermostability by around $5{ }^{\circ} \mathrm{C}$ as compared to AtRca $\alpha$ (Fig. 4.7C). Similarly, when the C-tail of AtRca $\alpha$ was incorporated to OsRca $\alpha$ (OsRca $\alpha$ AtTail), the chimeric mutant displayed around a $2{ }^{\circ} \mathrm{C}$ decrease in its thermostability as compared to OsRca $\alpha$. This indicates that the C-tail of AtRca $\alpha$ could not confer thermostability to OsRca $\alpha$ and swapping of C-tail negatively impacts the thermostability of the respective Rca (Fig. 4.7C). Altogether these results suggested that residues responsible for thermostability can largely be attributed to the nucleotide binding domain. Our observation was consistent with the previous study where a chimeric activase mutant consisting NBD of tobacco and Cterminal of arabidopsis (Tob-Arab) displayed tobacco like thermostability [13].

Additionally, our results indicate that ATPase, and more importantly, Rubisco activase activity of the chimeric mutants were similar to the wild-type Rca whose NBD it bears (Fig. 4.7D). For example, activase activity of RcaßAtNOsC was observed to be around $75-80 \%$ of AtRca $\beta$ and approximately 4 times higher than OsRca $\beta$, while RcaßOsNAtC retained $70-75 \%$ activase activity compared to OsRca $\beta$ and only $12-15 \%$ of the AtRca $\beta$ activity. Similarly, activase activities of AtRca $\alpha$ OsTail were also observed to be at a level more similar to AtRca $\alpha(60 \%)$ than OsRca $(8.5$ fold) while OsRca $\alpha$ AtTail possess $60-65 \%$ activase activity with respect to OsRca $\alpha$ and only $4-6 \%$ activity relative to AtRca $\alpha$.

Furthermore, RcaßAtNOsC maintained almost full ATPase activity (100\%) of AtRca $\beta$ (Fig. 4.7D), suggesting that the NBD of AtRca $\beta$ is important for high levels of ATPase activities. Consistently, ATPase activity levels of AtRca $\alpha$ OsTail were also observed at similar level to AtRca $(60-65 \%)$. This is expected as AtRca $\alpha$ OsTail also contains the NBD of AtRca $\alpha$ which we believe to be important for high levels of ATPase activities. These results were further supported by the low levels of ATPase activity recorded in our data for both RcaßOsNAtC and OsRca $\alpha$ AtTail as compare to AtRca $\beta$ and AtRca $\alpha$ respectively. Rca $\beta$ OsNAtC retained only $60 \%$ ATPase activity compared to AtRca $\beta$, while OsRca $\alpha$ AtTail meagrely possess only $12-15 \%$ activity relative to AtRca $\alpha$. Collectively, these observations suggest that both high activase and ATPase activity of the chimeric mutants mapped to the NBD. Thus, it was 
intriguing to further study the residues in the NBD region in an attempt to precisely identify the determinants of these features.

\subsection{ATPase activity of AtRca isoform was not enhanced in presence of DTT and thioredoxin-f of rice}

As described previously (section 3.3), ATPase activity of OsRca $\alpha$ could be modulated by the action of thioredoxin- $\mathrm{f}(\operatorname{trxf})$ in presence of a reductant such as DTT. Therefore we investigated the effect of rice thioredoxin-f on AtRca (Fig.4.8). As a control experiment, a 2-fold increase in ATPase activity of was observed for OsRca $\alpha$ in presence of thioredoxin and DTT (Fig. 4.8A). Contrary to this, ATPase activity of AtRca $\alpha$ was not stimulated in presence of rice thioredoxin and DTT (Fig. 4.8B). This suggests that redox regulation should involve specific intra-protein interactions plausibly between the residues of $\mathrm{C}$-tail and other domains of activase as reported earlier $[155,156]$.

Interestingly, we have observed that ATPase activity of AtRca $\alpha$ was reduced in presence of DTT and thioredoxin (Fig 4.8B). This behaviour was contradictory to existing literature available on redox regulation of Rca and also to our results with rice activase. Is it really possible that an inverse regulatory mechanism could exist between thioredoxin- $f$ and agave activase? However, the reduction in activity was not significant enough to rule out the probability of experimental error and it would be too early to make a statement at this point. Further investigation is needed to validate the outcome and if so then follow up study should be conducted in future to understand the mechanistic aspect of this observation. The preliminary attempts to purify recombinant thioredoxin-f of agave were not successful. In the near future, further attempts would be made to purify recombinant thioredoxin-f isoform of agave and utilize it to conduct similar experiments. This will be useful to explore if the specificity of thioredoxin- $f$ is a crucial factor or a different regulatory mechanism of regulation may exist for agave as compared to rice. 
A

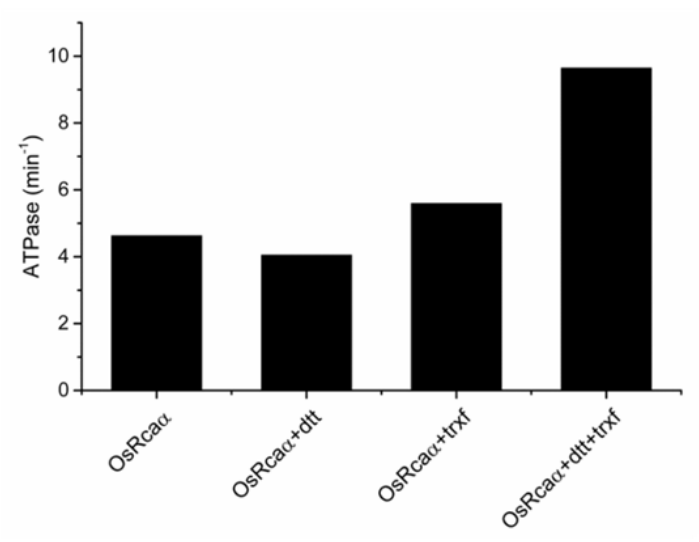

B

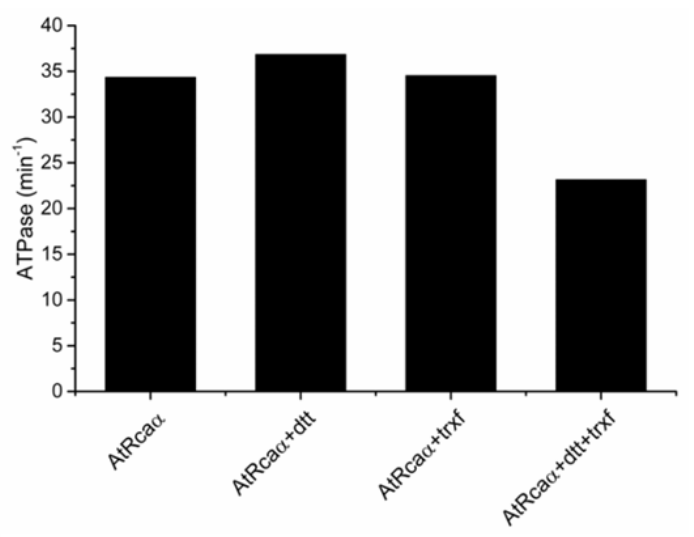

Fig. 4.8: Comparative effect of rice thioredoxin-f on ATPase activity of AtRcaa. Activases (A) OsRca, , (B) AtRca $(25 \mu \mathrm{M}$ each) were incubated with either $5 \mathrm{mM}$ dithiothreitol (dtt), $3.6 \mu \mathrm{M}$ rice thioredoxin-f (trxf) or both for 10 minutes prior to conducting assays. Assays were conducted at $25^{\circ} \mathrm{C}$ with $5 \mu \mathrm{M}$ Rca protomer, $1 \mathrm{mM}$ DTT and $0.72 \mu \mathrm{M}$ thioredoxin-f in the final reaction mix.

\subsection{Mutational analysis of various residues at NBD revealed that a single glutamate (217) to glutamine (E-Q) substitution confers high in vitro activase activity to OsRcaß}

In order to further identify the residues responsible for higher thermostability and activase activity of AtRca $\beta$, we initiated a comparative sequence-based mutagenesis study in the NBD of rice. Subsequently, site-directed point mutants were generated in the NBD of rice based on the sequence alignment between rice and agave Rca $\beta$ isoforms. Out of various uncommon residues, only the residues which are different in rice and both the isoform of agave were chosen (Fig. 4.9A). 


\section{A}

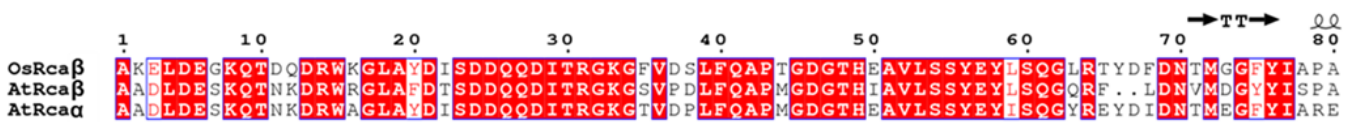

$\Delta \boldsymbol{\Delta}$

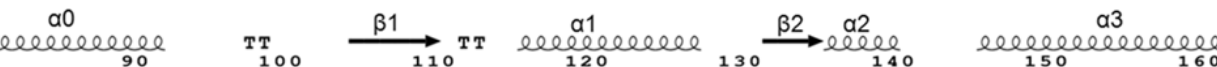

OsRCa $\beta$ FMDKLVVHIS SKNFMTLPNIKVP I ILGIWGGKGQGKSFQCELVFAKMGINP IMMSAGELES GNAGEPAKLIRQRYREAADI

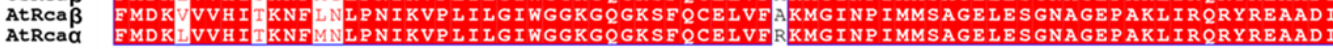
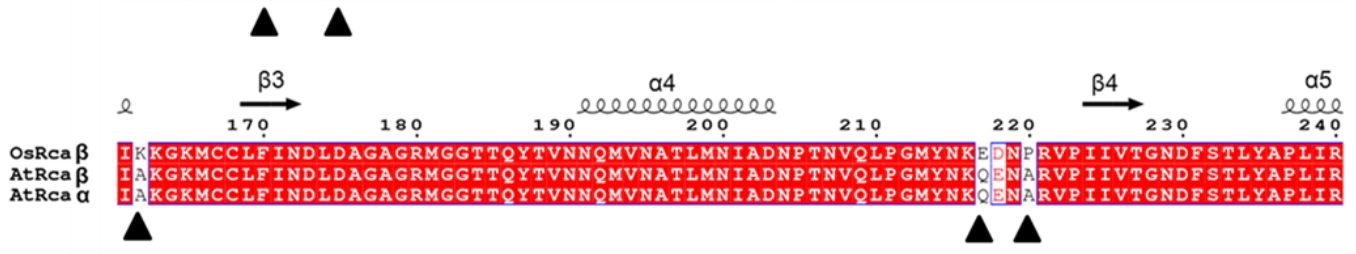

B

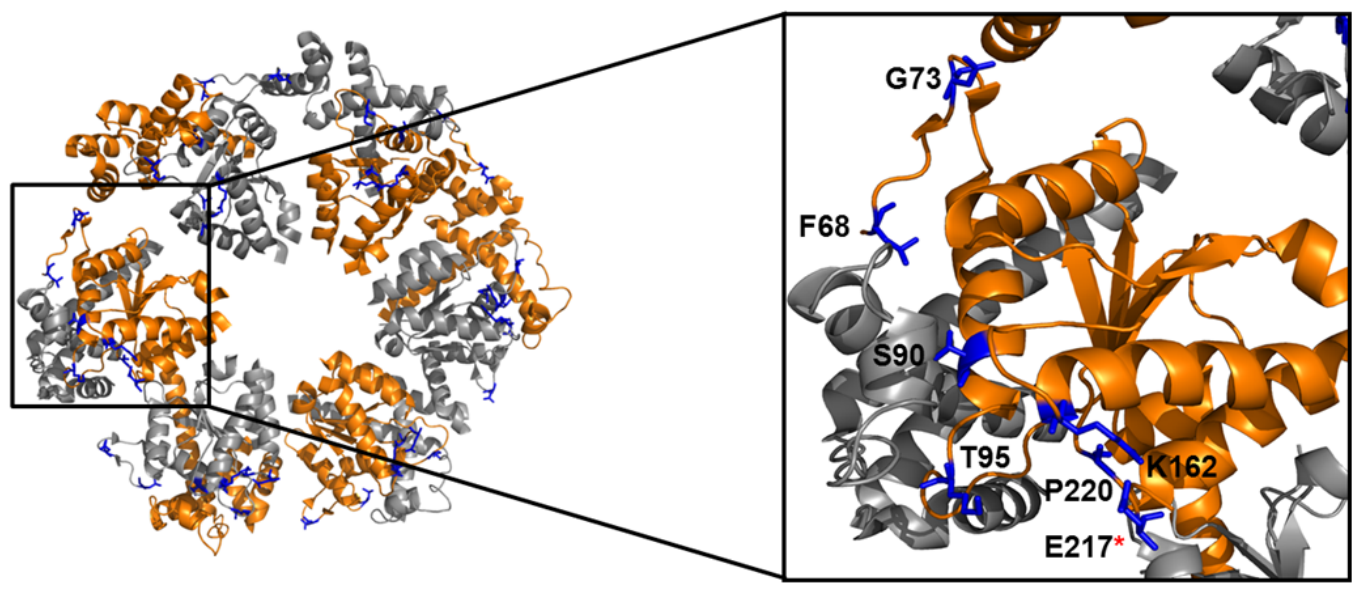

C
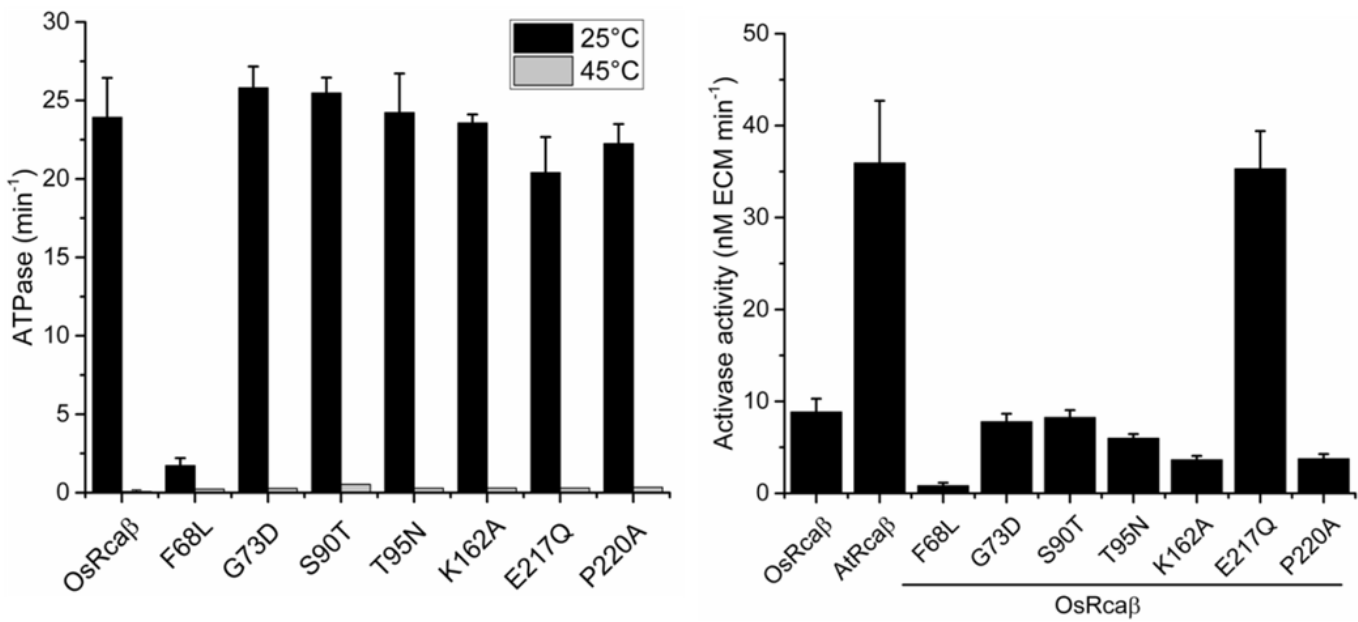
Fig. 4.9: Thermal and biochemical characterization of site-directed point mutants in NBD of rice. (A) Sequence alignment between rice and agave featuring NBD region highlighting mutated residues. (B) Ribbon representation of the hexameric model of tobacco Rca (PDB id: 3ZW6) (left) displaying the locations of the mutated residues in blue (right). *The position of E217 is not resolved in the structure and therefore the residue resolved adjacent to it (E219 in tobacco) has been displayed (C) ATPase assay was performed at $25{ }^{\circ} \mathrm{C}$, and after a 10 minute incubation at $45{ }^{\circ} \mathrm{C}$. (D) Biochemical characterization of the mutants. Rubisco activase activities of WT Rca isoforms measured at $25{ }^{\circ} \mathrm{C}$ with $5 \mu \mathrm{M}$ Rca protomer, $0.5 \mu \mathrm{M}$ ECM and ER (final concentration). Error bars indicate the standard deviations of mean values of three or more experiments.

Thermostability test on these point mutants revealed that all tested mutants possessed similar thermotolerance as OsRca $\beta$ and none of the point mutations could enhance its thermostability even to a slight extent (Fig. 4.9C). Biochemical characterization of the point mutant showed that all Rca mutants retained more or less similar base level of ATPase except F68L (Fig. 4.9C). The Rubisco activation activity of all the point mutants was also intact except F68L which only possessed $<10 \%$ of the activity, while K162A and P220A retained around 50\% of the activity. G73D, S90T, and T95N preserved $\sim 90 \%$ of the activity compared to WT. Interestingly, E217Q attained a significantly much higher activation activity ( $\sim 4$ fold) as compare to OsRca $\beta$ and conferred AtRca's high activation rate to OsRca (Fig. 4.9D).

Examination of the structure of Rca from tobacco [129] revealed that area around this residue was not resolved but appears to be a part of disordered element. This flexible loop is predicted to be present on the surface and in close proximity to helix 9 (within 17-22 $\AA$ ) of the adjacent subunit (Fig. 4.10A) which is responsible for recognition of Rubisco in plants $[129,132]$. Therefore, we hypothesized that this residue or loop is crucial for Rubisco remodelling and resulting charge difference due to mutation changes the extent of interaction at the Rubisco interacting sites. However, other variants of this mutant such as E217A, E217K possessed the similar phenotype (Fig. 4.9D). This suggested that this residue may not be directly involved in the proteinprotein interaction. Instead, mutation of this residue may cause conformational changes in and around the interacting region, which somehow results in better recruitment or a stronger interaction with Rubisco. 


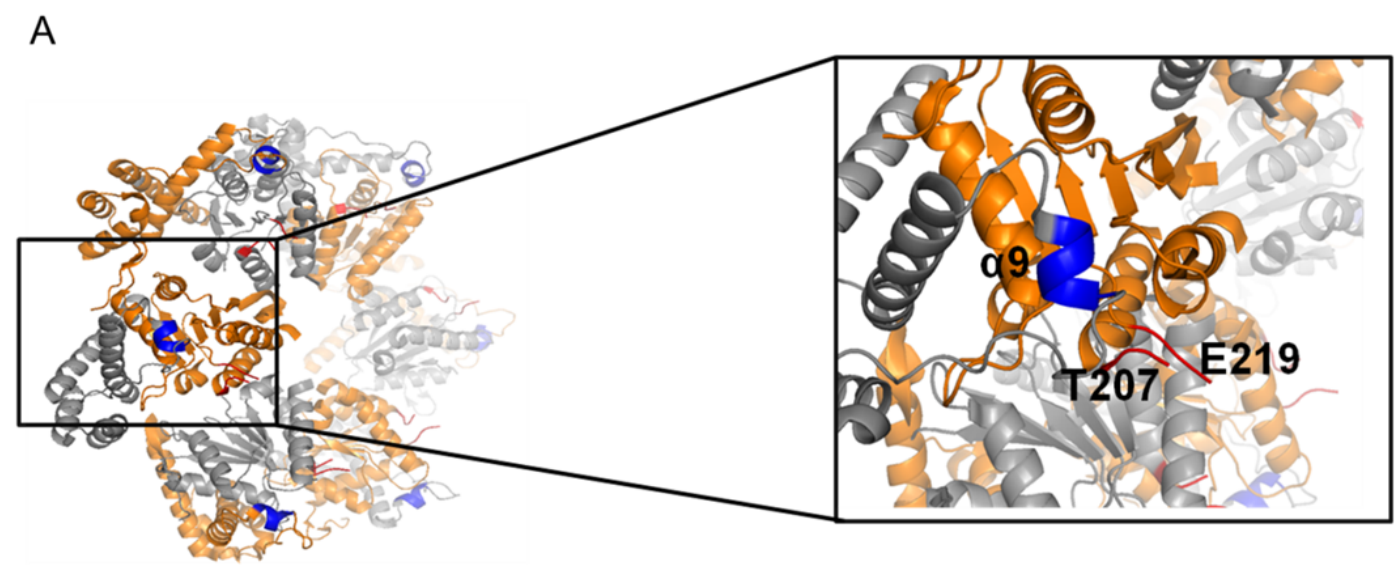

B

C
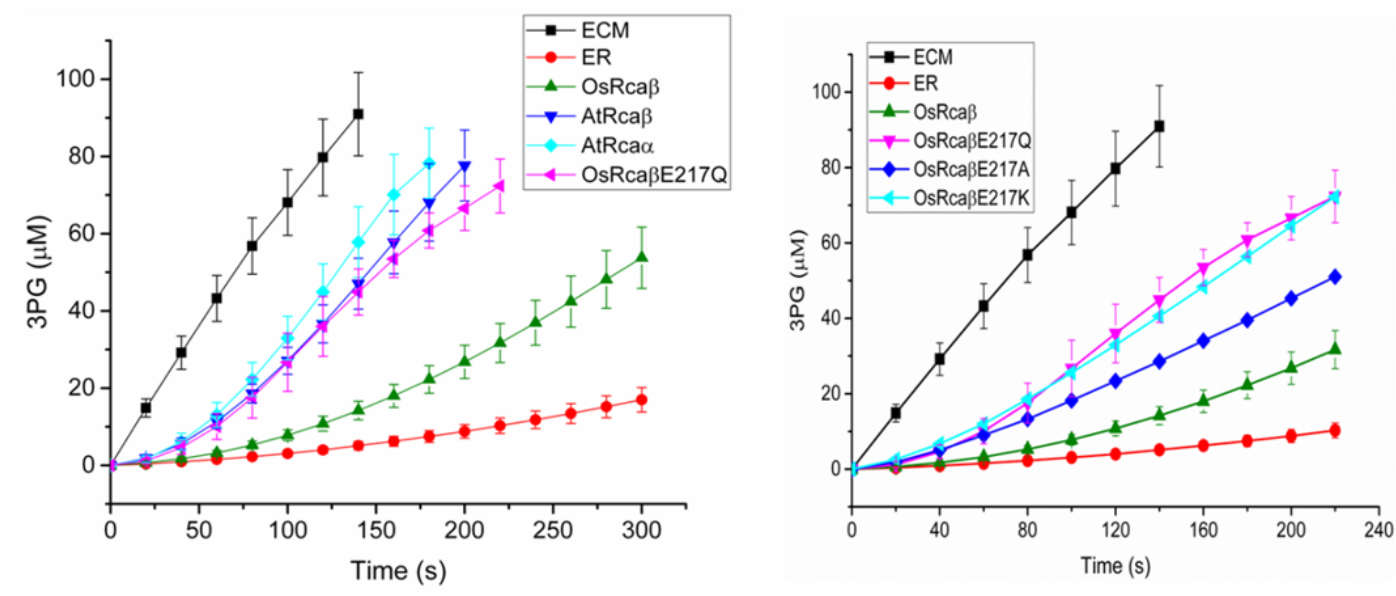

Fig. 4.10: Characterization of OsRcaßE217Q. (A) Ribbon structure of hexameric model of tobacco Rca (PDB id: 3ZW6) highlighting disordered loop which consists the mutated residue (red), along with helix $9(\alpha 9)$ region (blue) responsible of Rubisco recognition in plant. The numbering was as per tobacco. Rubisco activation assays for OsRcaßE217Q along with WTs Rca from rice and agave (B), and other E217 variants (C). Assays were conducted at $25{ }^{\circ} \mathrm{C}$ with $5 \mu \mathrm{M}$ Rca protomer concentration and $0.5 \mu \mathrm{M}$ ER and ECM. Error bars indicate the standard deviations of mean values of three or more experiments.

From the above data it can be concluded that mutational analysis of the NBD region could not locate the key residues responsible for thermostability. However a single glutamate to glutamine (E217Q) substitution can impart agave-like properties in terms of Rubisco reactivation activity (Fig. 4.10B). It was a promising outcome which prompted us to further study this unidentified loop in order to precisely determine its role in Rubisco-Rca interaction. 


\subsection{Mutational analysis of the newly identified surface loop residues revealed a key lysine (216) important for Rubisco-Rca interaction}

Following the previous observation, single point mutants were generated (Fig. 4.11A) to test the impact of mutation in this surface loop in terms of ATPase and Rubisco activation activity. Mutating the aromatic tyrosine residue (Y214A) led to around $80 \%$ loss of ATPase activity and an almost complete loss of Rubisco activase activity (Fig. 4.11C). Mutation of asparagine residues in the loop (N215A \& N219A) led to reduction of both ATPase as well as Rubisco activase activity. A reduction of around $45 \%$ and $80 \%$ in ATPase rate and decline of approximately $78 \%$ and $65 \%$ in Rca function was observed for N215A and N219A respectively. Mutating aspartate-218 to alanine was tolerated. However, mutating the lysine residue (K216A) adjacent to the glutamate residue (E217) characterized above led to loss of $85-90 \%$ of the Rubisco activation activity, while still retaining $\sim 70-75 \%$ of the ATPase activity. This was highly indicative of a possible role of this lysine residue in the Rubisco-Rca interaction. Creating a charge switch mutation of this variant (K216E) displayed an even stronger phenotype with almost complete abolition of activase function, while only maintaining $\sim 50 \%$ of the ATPase rate (Fig. 4.11C).

Taken together these observations argued for the importance of this surface loop, especially the lysine-216, in Rubisco-Rca interaction. Since structurally this loop appears to be close to helix 9 (Fig. 4.10A), it is quite possible that this loop might either be directly involved in Rubisco-Rca protein interaction through docking or might play an important role in conformational remodelling of the active site of Rubisco. 
A
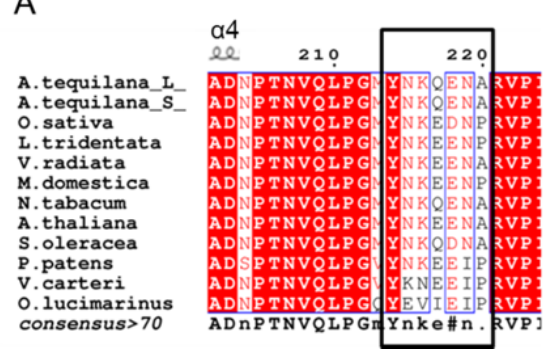

B

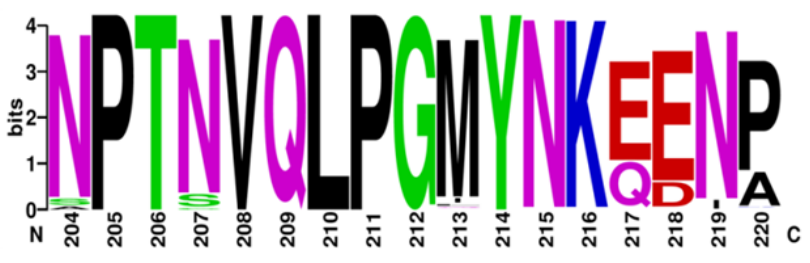

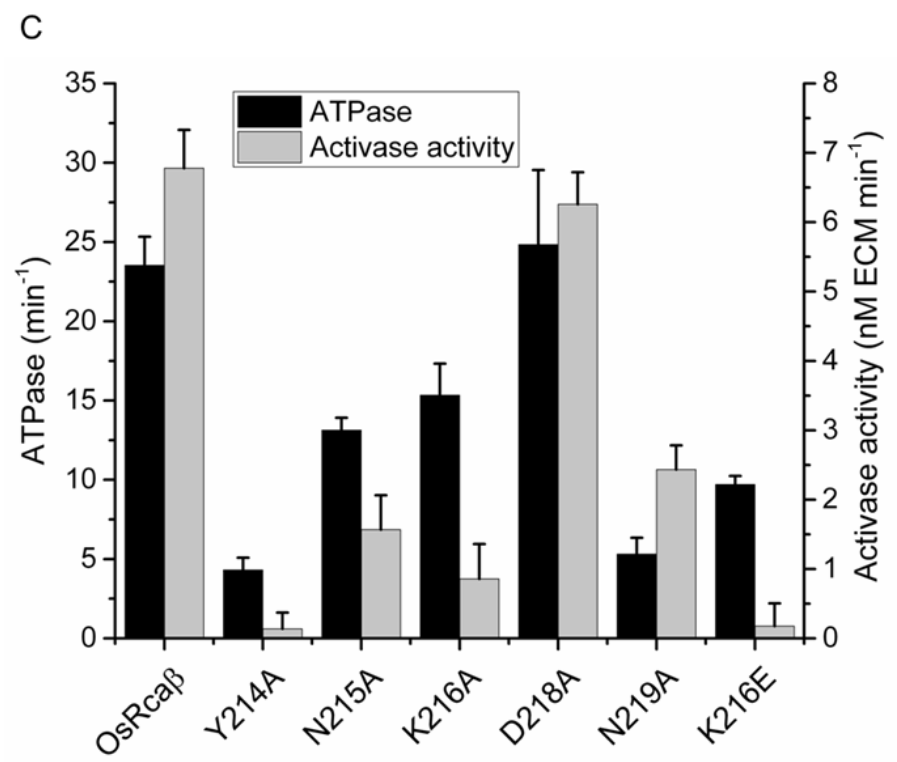

Fig. 4.11: Characterization of the newly identified surface loop residues. (A) Sequence alignment between rice and agave of the region targeted for mutational study highlighting the mutated residues. (B) Web logo representation [232] of sequence conservation for Rca residues in and around the surface loop (residues 207-217). Sequence logo was created using an alignment of Rca sequences from more than 200 plant species. Size of the letter denotes the level of conservation for particular residue proportional to other. (C) Biochemical characterization of the residues in the loop. ATPase and Rubisco activation assays were performed at $25^{\circ} \mathrm{C}$ with $5 \mu \mathrm{M}$ Rca protomer, $0.5 \mu \mathrm{M}$ ECM and ER. Error bars indicates the standard deviations of mean values of three or more independent experiments.

The web logo representation [232] of the sequence elements of this loop demonstrates high conservation level among Rca family in plants except for E217 and D218 (Fig. 4.11B). Glutamic acid (E) at position 217 was observed to be substituted by glutamine (Q) in most of the cases including for tobacco, agave and spinach (Fig. 4.11A and B). Considering the results for E217Q mutants in our study (section 4.7) it could be speculated that this substitution might be an affinity switch among Rca in plants. A comparative biochemical characterization of the other Rca homologues carrying this hypothetical switch needs to be performed in future. 
Unavailability of a full length atomic model of Rubisco activase until now, even after several attempts, limits our understanding in predicting the exact mechanism. Solving a full length structure of Rubisco activase would assist in planning other structurally guided mutations to understand the activation process comprehensively. Also additional Rca homologues should be considered for bioprospecting and careful biochemical characterization should be carried out to achieve mechanistic insights and identification of candidate proteins for engineering thermostable activase. Considering the data from this study other CAM plants could be a potential source for such exploration process due to their vast diversity and innate physiological properties. Here we predict that, since CAM has evolved convergently on multiple occasions $[42,233,234]$, different molecular solutions to thermostability may have evolved. 
5. Generation of a rice Rubisco activase molecular toolbox and towards an improved mechanistic appreciation 
The mechanism by which Rubisco activase remodels Rubisco enzyme to release sugar phosphate inhibitors is poorly understood in spite of several mutational studies and emerging structural information. Recently, strong evidence has been produced that red-type Rca (CbbX) functions by threading the C-terminus of the Rubisco large subunit through the central pore of the disc-shaped oligomer [104, 114]. However, an analogous mechanism for the plant system appears unlikely, as the C-terminus of the plant Rubiscos is significantly shorter. In addition, a key tyrosine residue responsible for the threading in the CbbX pore loop 1 segment is absent in the plant activases [158]. In the past several mutagenesis studies have been conducted towards understanding the mechanism of Rubisco regulation by Rca. Based on these studies some of the critical residues involved in ATP binding, hydrolysis and Rubisco activation were identified (reviewed by $[8,47]$.

The results from these studies are taken as basis in this section and discussed in terms of their locations/sub-domains in Rca structure. The mutational works performed on activases in the past were mostly the outcomes of sequence homology models of Rubisco activase to the other AAA+ proteins [8] due to non-availability of a model of Rubisco activase structure. Here, we have utilized both the knowledge from earlier mutagenesis studies and the newly gained structural information on Rubisco activases $[129,131]$ to demonstrate and compare the effect of already known mutants in rice. We have also introduced new structurally guided mutation to further probe their function in terms of ATP hydrolysis and Rubisco activation. Unfortunately, the three currently available published crystal structures of Rubisco activase are incomplete. Out of them the comprehensive structures of arabidopsis and tobacco Rca are lacking the N-terminal domain, the pore loop regions and the regulatory $\mathrm{C}$-terminus of the long isoforms [129-131]

Furthermore, the function of activase in terms of subunit-subunit interaction and cooperativity is also mostly elusive. Subunit mixing or mutant doping strategy is one of the promising ways to overcome this. A mutant doping strategy is often used to determine the relative contribution of the individual mutant subunits towards ATPase and remodelling activity in AAA+ proteins by biochemically analyzing the properties of mutant/wild-type subunit mixtures [193, 194, 235]. For example, the relative ability to tolerate ATPase inactive subunits reveals whether a concerted or stochastic 
mechanism is employed in function [236] as previously shown (section 3.5.1). Rubisco activase also demonstrates ability to rapidly exchange subunits as reported earlier $[129,163,164]$ and in the present study (section 3.5.1) and is amenable to the mutant doping strategy. In this section, we have employed this strategy to determine the contribution of individual subunit towards Rubisco activation and measure the relative importance of the tested residues.

In this strategy, usually ATP hydrolysis or substrate binding impaired mutant subunits are mixed with wild-type subunits in variable ratios, keeping the final protein concentration constant. Random mixing between WT and mutant subunits would lead to formation of heterogeneous population of hetero-oligomeric ensembles (Fig. 5.1A). This generates a variable probability distribution of incorporation of particular number of mutant subunit in hetero-oligomer as a function of fraction of mutant subunit present in the mix (WT: mutant) which can be modelled using a binomial distribution (Fig. 5.1A, also see 2.7.10.3). Therefore, for any specified percentage of mutant subunits the probability distribution of hexamers containing $0,1,2,3,4,5$ or 6 mutant subunits can be derived as shown in Fig. 5.1A.

This distribution is then used to predict how activity could be inhibited at different mutant-wild-type ratio. By plotting a curve between theoretical relative activities against \% of mutant, theoretical activities at a particular ratio of WT/mutant could be determined. Different curves are obtained, based on the specific number of mutant subunits (1-6) required to abolish activity of the whole hexamer (Fig. 5.1B) [193, 194, 236]. 
A
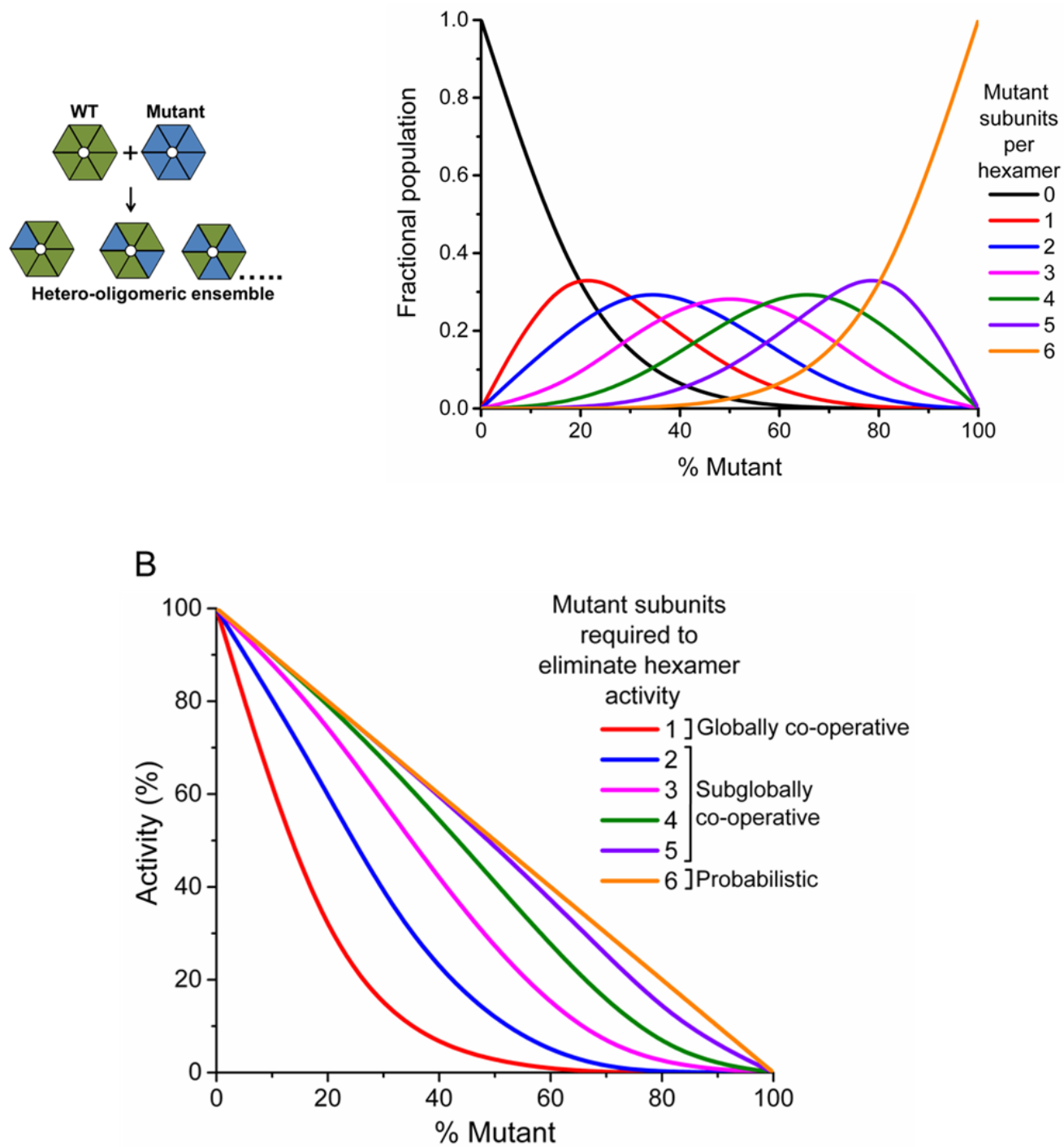

Fig. 5.1: Theoretical model to predict the effect of subunit doping. (A) Theoretical populations of Rubisco activase hetero oligomeric ensembles containing various numbers of mutant subunits (0-6) as function of the fraction of mutant subunit present in the mixture as represented by different colours. (B) Curves representing the theoretical activity against the \% of mutant subunits present, when a particular number of mutant Rca subunits (1-6) are required to abolish the activity of the Rca hexamer.

\subsection{Subunit doping experiment with Walker B mutants indicates strong cooperativity between subunits}

Using the above model, we can determine how Rubisco activation activity is inhibited at various WT to mutant ratios. Based on that analysis, we can state whether subunit coupling within activase oligomers follows a probabilistic (non-cooperative), sub- 
globally cooperative or globally cooperative model by comparing our data with the theoretical plots (Fig. 5.1B). These outcomes were based on the hexamer as the functional unit. Consequently, if subunits within the hexamer are independent and follow the probabilistic model, it means the cooperativity is dispensable (noncooperative effect) and only one WT subunit per hexamer will be sufficient for activity. In this case, activity should decline in a linear manner with increasing concentration of the mutant subunits in the mix. On the other hand, if subunits are coupled, then a specific number of mutant sub-units are required to abolish the activity of the hexamer, which would be consistent with a subglobally cooperative (if two to five mutant subunits per hexamer are required to eliminate activity) or a globally cooperative model (if only one mutant subunit per hexamer ablate activity). In a globally cooperative model there would be a rapid drop in activity and therefore a steep curve is expected, while in case of a subglobally cooperative behaviour an intermediate curve is anticipated (Fig. 5.1B) [193, 194].
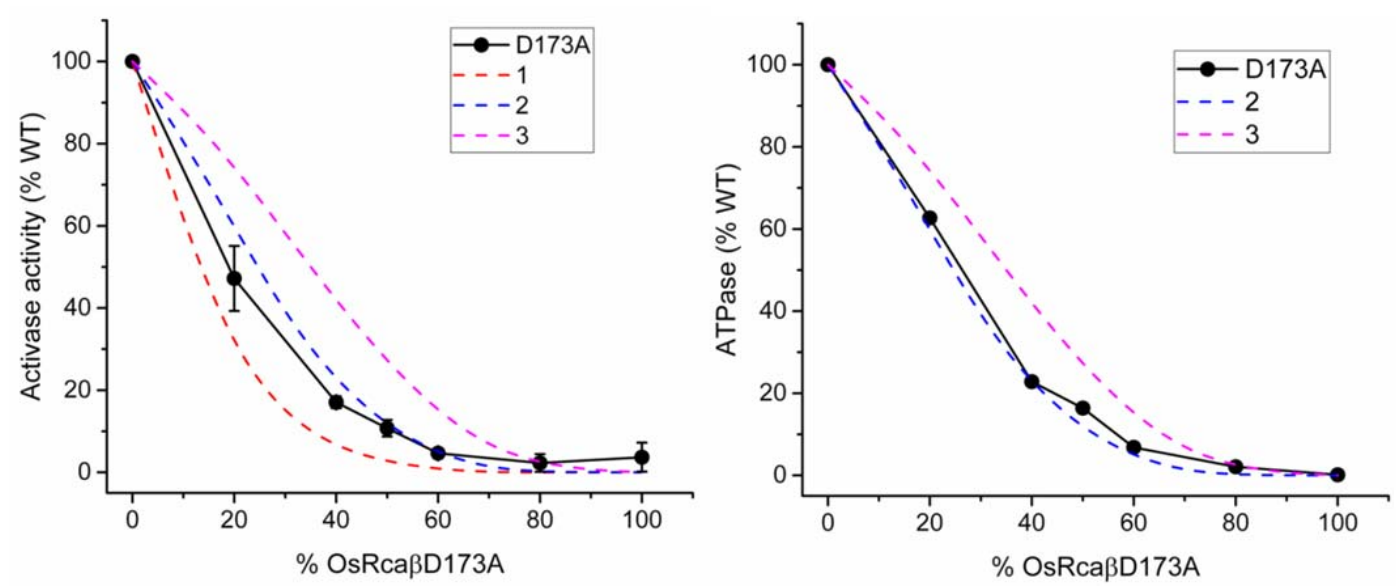

Fig. 5.2: Titration of WT $\beta$-Rca with increasing concentration of ATPase inactive Walker B (D173A) mutant. WT active $\beta$ isoform was pre-incubated with the OsRcaßD173A at 258-272 $\mu \mathrm{M}$ Rca protomer concentration range for $10 \mathrm{~min}$. Rubisco activation assay (left) and ATPase assay (right) were performed by maintaining $5 \mu \mathrm{M}$ activase protomer concentration in the final reaction mix at $25{ }^{\circ} \mathrm{C}$. Activase and ATPase activity was calculated considering WT activity as $100 \%$. Dotted lines indicate the theoretical activity curves if respective number of subunits abolishes hexamer activity. Error bars indicate the standard deviations of the mean values of three or more independent experiments. 
For instance if we take an example of ATPase inactive Walker B mutant (D173A), which was earlier shown to be completely ATPase inactive and followed a subglobally cooperative mechanism of ATP hydrolysis (section 3.5.1 and Fig. 5.2), was also found to be a strong mutant in terms of abolishing Rubisco activase activity in mixing with WT active subunits (Fig. 5.2). OsRcaßD173A itself was completely incapable of activating inhibited Rubisco by its own ( $>95 \%)$. The titration curve with increasing WT to mutant ratio revealed a significant drop of around 50\% of maximal activase activity even at very low proportion (20\%) of Walker B mutant. Subunit mixing at an equimolar ratio led to almost $80 \%$ loss of activity. Similarly a drop of $40 \%$ ATPase activity was observed at $20 \%$ mutant concentration (Fig. 5.2). This suggests strong inter-subunit coupling with respect to ATPase as well as Rubisco activation activity for Walker B mutant (D173A) of Rubisco activase in rice. Previously, a similar effect in terms of subunit interaction has been demonstrated for ATPase deficient mutants. For instance, addition of Walker B and D231R mutant with wild-type tobacco activase caused a reduction of $40 \%$ and $80 \%$ ATPase activity respectively whereas a drop of $75 \%$ activase activity has been reported in case of the K247R mutant $[163,164]$.

Mathematical analysis of our data using the theoretical curves (Fig. 5.1B) corresponds to 1-2 mutant subunits being sufficient to abolish the activase activity of whole hexamer, while 2-3 mutant subunits are enough to ablate ATPase function. Thus, in this case Rca hexamer must contain at least five ATPase functional subunits to catalyze Rubisco remodelling, while a minimal four subunits are necessary for ATPase function. It could also be stated that Rca subunits utilize a highly coupled sub-globally cooperative mechanism to hydrolyse ATP for substrate remodelling and multiple subunits within the hexamer should work together to carry out its function in an efficient manner.

In other homologous activase systems like CbbX and CbbQO [114, 115] also, Walker B mutants was found to be deficient in ATPase as well as activase function. However, the type of subunit cooperativity involved in their functioning was not analyzed in above studies. Walker B mutation in non activase AAA+ system such as ClpX, which follows probabilistic model for ATP hydrolysis displays complete loss of ATPase 
function and ability to unfold and translocate its substrate while allowing substrate binding $[237,238]$.

The double Walker B (DWB) mutation among class 1 AAA+ proteins such as ClpB and Hsp104 which possess two Walker B sites also abolished ATPase and luciferase reactivation function $[193,194,239,240]$. A strongly coupled mechanism in terms of ATPase and chaperone activity was also reported for the DWB mutant of ClpB, similar to our case $[193,194]$. On the other hand, Hsp104DWB follows a probabilistic mechanism for ATPase hydrolysis like ClpX but sub-globally cooperative model for luciferase reactivation activity [193]. However, variations in data have been reported by different groups, possibly implying that these proteins may exhibit operational plasticity in ATP utilization depending on the substrate and requirement of other assisting chaperones to determine the extent of cooperativity [240, 241].

\subsection{Validating the phenotype of key OsRea variants, studied previously in other plant Rca systems}

The effect of various mutations on ATPase and Rubisco activation activity has been investigated earlier for Rubisco activase of spinach and tobacco $[129,159,160]$. The

importance of the N-terminal domain has been reported in Rubisco activation as a putative substrate adaptor domain like other AAA+ proteins [128, 242, 243]. In agreement with the finding by van de Loo and Salvucci (1996), mutation of the conserved tryptophan 15 to alanine (W15A) in the N-terminal domain of rice Rca resulted in unaltered ATPase activity but a $>90 \%$ loss in Rubisco activation (Fig. 5.3). This indicates its involvement in engaging substrate through hydrophobic interactions with Rubisco. However, in contrast to the result reported for tobacco [129], the Nterminal truncation of 66 residues $(\Delta \mathrm{N} 67)$ resulted in a significantly elevated ATPase rate ( $\sim 1.7$ fold) and around $\sim 30 \%$ of residual Rubisco activation activity (Fig. 5.3). In tobacco, the equivalent truncation led to a normal ATPase hydrolysis rate and an almost complete loss of Rubisco activation [129]. 


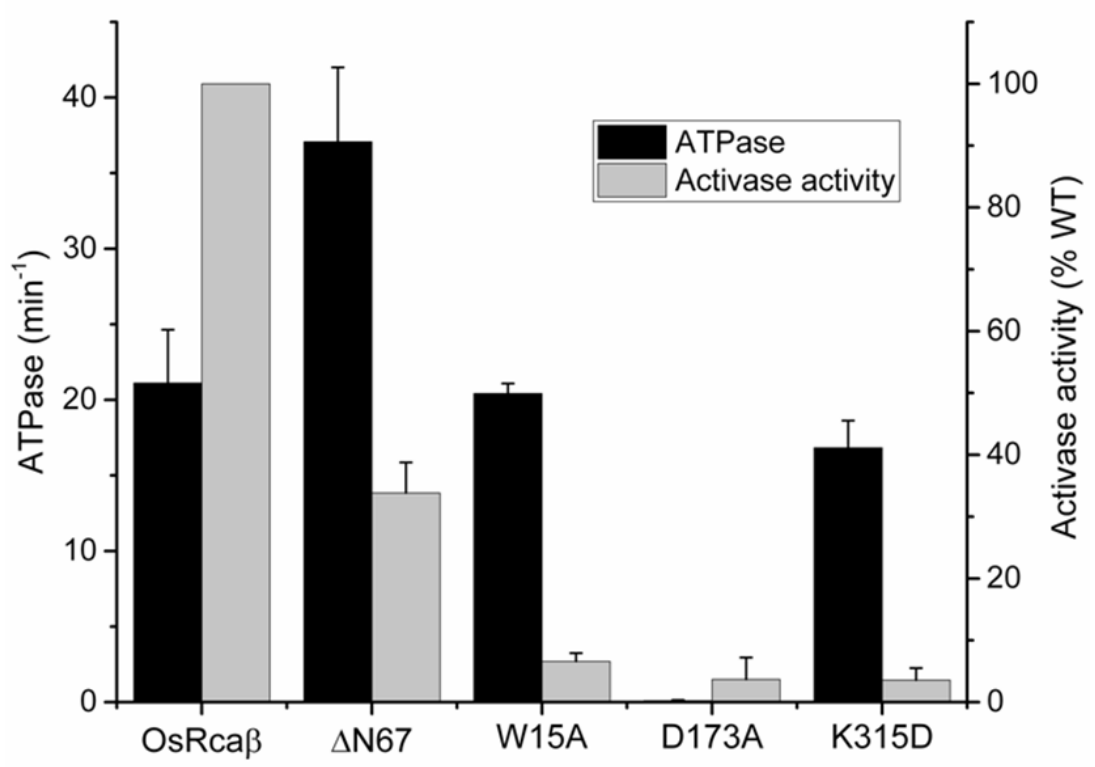

Fig. 5.3: Characterization of Rubisco activase mutants. ATPase and Rubisco activase activities of WT Rca ( $\beta$ isoform), N-terminal truncation $(\Delta \mathrm{N} 67)$, point mutations W15A (N-terminal), K315D (specificity helix), and D173A (Walker B) as indicated. Assays were performed at $25^{\circ} \mathrm{C}$ with $5 \mu \mathrm{M}$ activase protomer and $0.5 \mu \mathrm{M}$ inhibited Rubisco (ER). Error bars indicate the standard deviations of the mean values of three or more independent experiments.

A small helical insertion (5 residues) in the structure of Rca called helix $9(\alpha 9)$ is positioned on the periphery of the hexameric ring [129]. Mutation of the residues aspartate 316 and leucine 319 to lysine and valine respectively (tobacco) at helix 9 reversed the specificity of Rubisco activase from Solanaceae Rubisco to the Rubisco of non-solanaceous plants $[132,168]$. Consistently, we found that mutating the lysine at 315 to aspartate $(\mathrm{K} 315 \mathrm{D})$ in rice resulted in a slightly reduced ATPase rate $(\sim 80 \%)$, but almost no Rubisco activation activity (Fig. 5.3). Thus, this helix must play an important role in substrate recognition and was crucial for Rubisco activation in plants.

\subsubsection{Rubisco interaction site mutants display variable levels of importance in context of oligomer function}

The results in the previous section reconfirmed the important effect of a number of Rca features, previously reported for homologous systems. In order to determine the relative contribution of individual residues to activase function in context of the 
oligomer, we initiated systematic subunit-mixing/mutant doping experiments. These experiments are designed to further clarify how Rubisco activase subunits engage and remodel Rubisco, which is currently poorly understood.
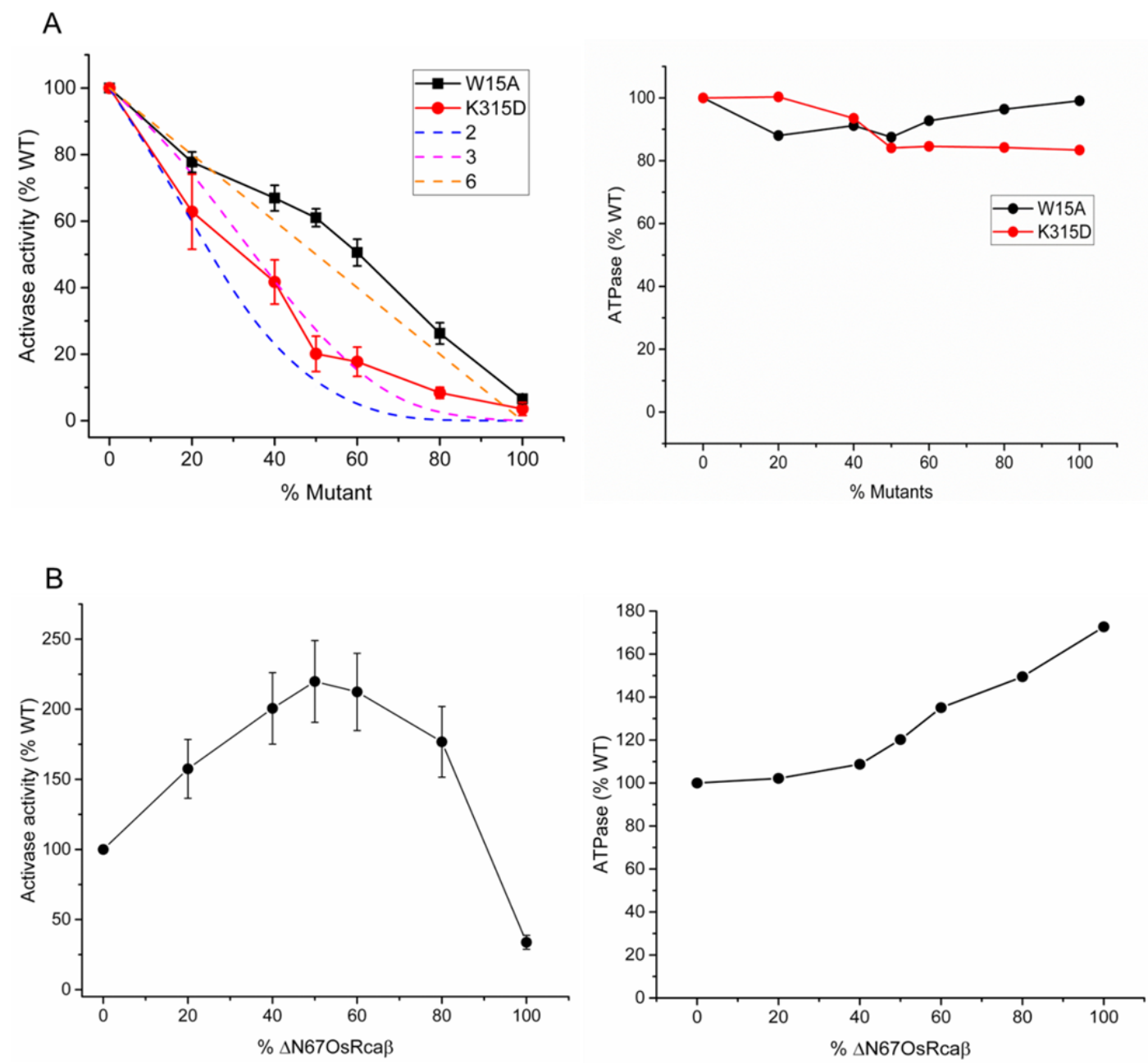

Fig. 5.4: Titration of WT $\beta$-Rca with increasing concentration of interaction site mutants. WT active $\beta$ isoform was pre-incubated with mutants: W15A, K315D (A) and $\Delta$ N67 (B) for 10 min to yield the desired proportion. Pre-incubation was done at 240-360 $\mu \mathrm{M}$ (W15A), 330-390 $\mu \mathrm{M}$ (K315D) and 250-360 $\mu \mathrm{M}(\Delta \mathrm{N} 67)$ Rca protomer concentration. Rubisco activation assay (left) and ATPase assay (right) were performed by maintaining $5 \mu \mathrm{M}$ activase protomer concentration in the final reaction mix at $25{ }^{\circ} \mathrm{C}$. Activase and ATPase activity was calculated considering WT activity as $100 \%$. Dotted lines indicate the theoretical activity curves if respective number of subunits abolishes hexamer activity. Error bars indicate the standard deviations of the mean values of three or more independent experiments.

Mixing of the W15A variant (N-terminal domain) with WT Rca in increasing proportions resulted in a roughly linear reduction in activase activity (Figure 5.4A). Assuming the hexamer as the functional unit, the data indicates that an activase 
hexamer can tolerate high number of inactive mutant subunits (W15A) and even a single WT subunit per hexamer is sufficient to remodel Rubisco. The high tolerance of WT Rca to W15A subunits suggests that the protein-protein interaction mediated by this residue need not to be present for all six subunits of activase (Fig. 5.1B and 5.4A). This supports a recruitment and/or orientation function of this residue, rather than an active mechanistic role in Rubisco remodelling. The W15A mutant has an approximately similar ATPase activity as WT and subunit mixing with WT almost has no effect on the ATPase activity of the heterocomplexes (Fig. 5.4A). This indicates that the observed pattern in case of activase activity is not affected by the ATPase rate during experiment.

In contrast, the specificity helix charge switch mutant (K315D) showed high cooperativity in terms of activase activity. The data corresponded to a situation where 2-3 mutant subunits abolished hexamer activity (Fig. 5.1B and 5.4A). If two mutant subunits can abolish the activity of Rca hexamer then four Rca subunits are not enough for activity, hence five are needed. Similarly if three mutant subunits can inactivate Rca hexamer then three subunits are not sufficient for activities, hence four are needed. Thus 4-5 WT subunits per hexamer are required for substrate engagement. This suggests that Rubisco engagement in plants via helix 9 utilises a sub-globally cooperative mechanism to remodel Rubisco by utilising multiple subunits within hexamer to be functional at a time. This might involve interaction with more than one recognition site on the surface of different Rubisco large subunit. Subunit doping between K315D and WT Rca did not alter the ATPase activity of the heterocomplexes (Fig. 5.4A). However, the problem here is that the charge switch used in this case is from a positively charged residue to a negatively charged one, which would cause electrostatic repulsion at the interaction site and thus complicates our analysis. Therefore, for a clearer answer, the K315A substitution mutant should be analyzed in future which might generate less drastic changes. In any case, the observed result suggests that multiple helix 9 moieties of the oligomer interact with Rubisco during remodelling.

Mixing experiments with the N-terminal domain (NTD) deletion mutant $(\Delta \mathrm{N} 67)$ resulted in a counterintuitive outcome. Earlier, we observed that mutation at Nterminal domain (W15A) resulted in a linear decline in activase activity of Rca 
hexamer. In contrast, activase activity of WT Rca was stimulated by the $\Delta$ N67 mutant (Fig. 5.4B). The $\Delta \mathrm{N} 67$ by itself possessed around $\sim 30 \%$ of activity, but led to an enhanced activase activity compared to WT, when incorporated at any proportion. A more than 2-fold increase in the activity was observed at an equimolar mixing concentration. Even at $80 \%$ of mutant concentration, the mix maintained an around 1.5-1.7 fold higher activation rate than wild-type subunits. This result suggests that, like for W15A, only 1 or two NTD subunits are required for interaction with Rubisco and the higher activation rate may be attributed to the higher ATPase rate of the NTD deletion subunits (Fig. 5.4B). This observation also compels us to hypothesize that the ATP hydrolysis rate may be the dominant determinant of activation rather than the Rubisco interaction in this case. Probably, this combination should be working in a complimentary way, where required interaction sites in the oligomeric mixture are provided by small number of WT Rca subunits but the overall high ATPase rate of the oligomer may be responsible for better activation. Similar to our observation, a NTD mutant of the yeast chaperone Hsp104 was also found to possess a higher ATPase rate but reduced disaggregase activity and exhibit a stimulatory effect with other mutants as found for Rca in our study [240, 244]. However the effect of mixing NTD mutant subunits with WT Hsp104 was not reported in these studies.

Next, we performed subunit mixing experiments between $\Delta \mathrm{N} 67$ and other mutants to determine whether the NTD mutant could also enhance the activity of other activase mutants when included in a hetero-oligomeric complex. These were 1) the Walker B mutant (D173A) which can provide a functional N-terminal domain but no ATPase activity, 2) the N-terminal domain mutant (W15A) which could provide ATPase function but lacks a functional N-terminal domain and, 3) the Helix 9 mutant K315D responsible for species specificity, which can offer both ATPase activity and required $\mathrm{N}$-terminal domain, but is impaired at another Rubisco interaction site (Fig 5.5A and B).

In the case of D173A and W15A, the stimulatory trend seen for the WT subunit was not observed (Fig. 5.5B). Adding the D173A mutant to $\triangle \mathrm{N} 67$ resulted in a drastic reduction of activase and ATPase function similar to that seen when mixing to wildtype. A drop of around $60 \%$ in ATPase and activase activity was observed compared to $\triangle \mathrm{N} 67$ in the presence of low concentration of D173A (20\%). In contrast, at low 
proportion of W15A, activase activity of the composite oligomer was reduced and almost resembled the function of the W15A variant alone (100\%) at all proportions. Since, the ATPase activity of this oligomeric mixture is not strongly perturbed (Fig. $5.5 \mathrm{~B}$ ), the effect should be the outcome of a missing interaction site due to unavailability of a functional $\mathrm{N}$-terminal domain.

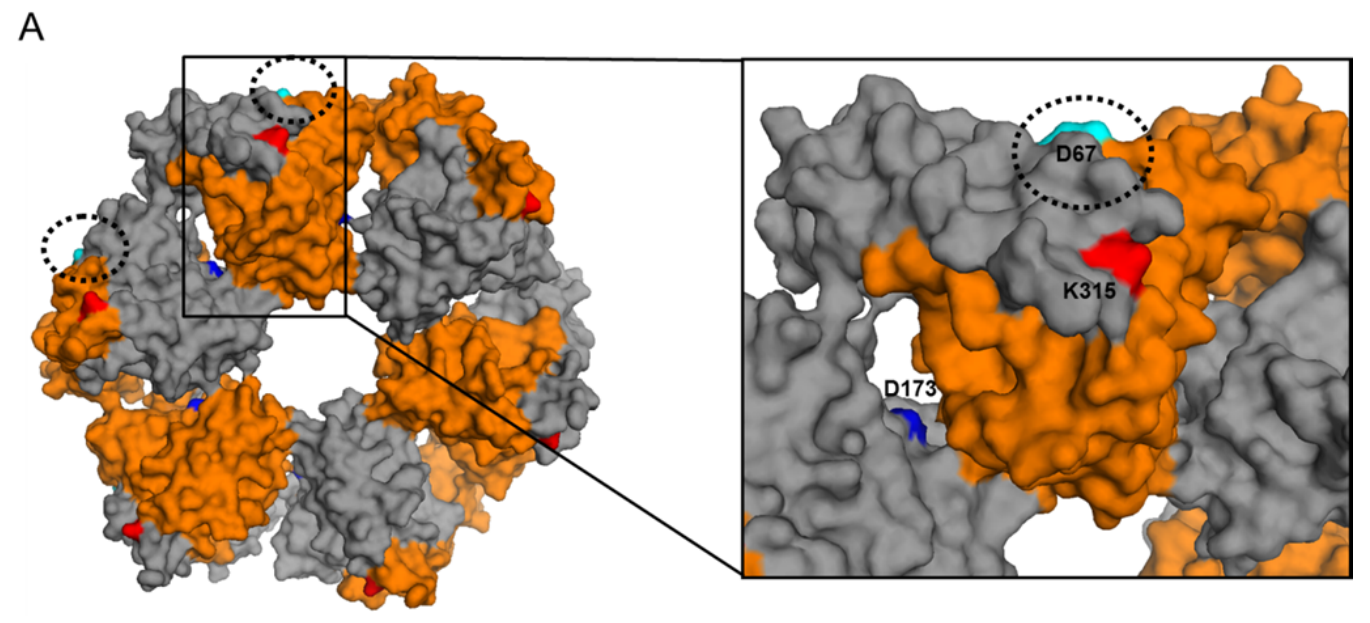

B
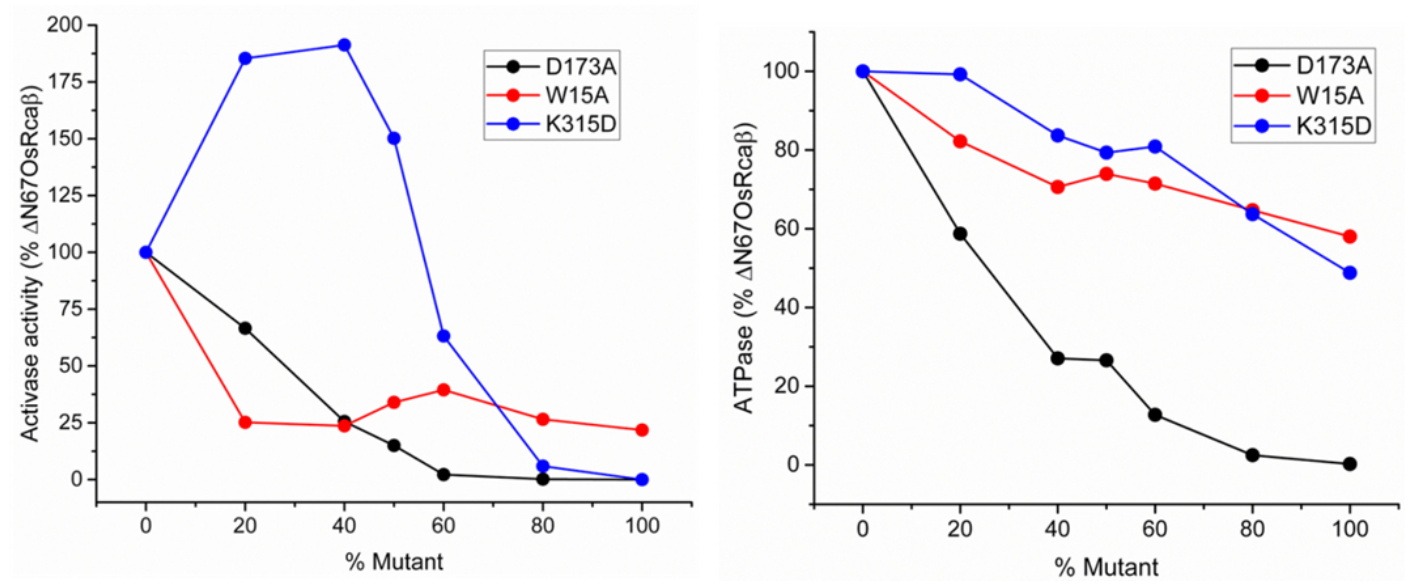
C

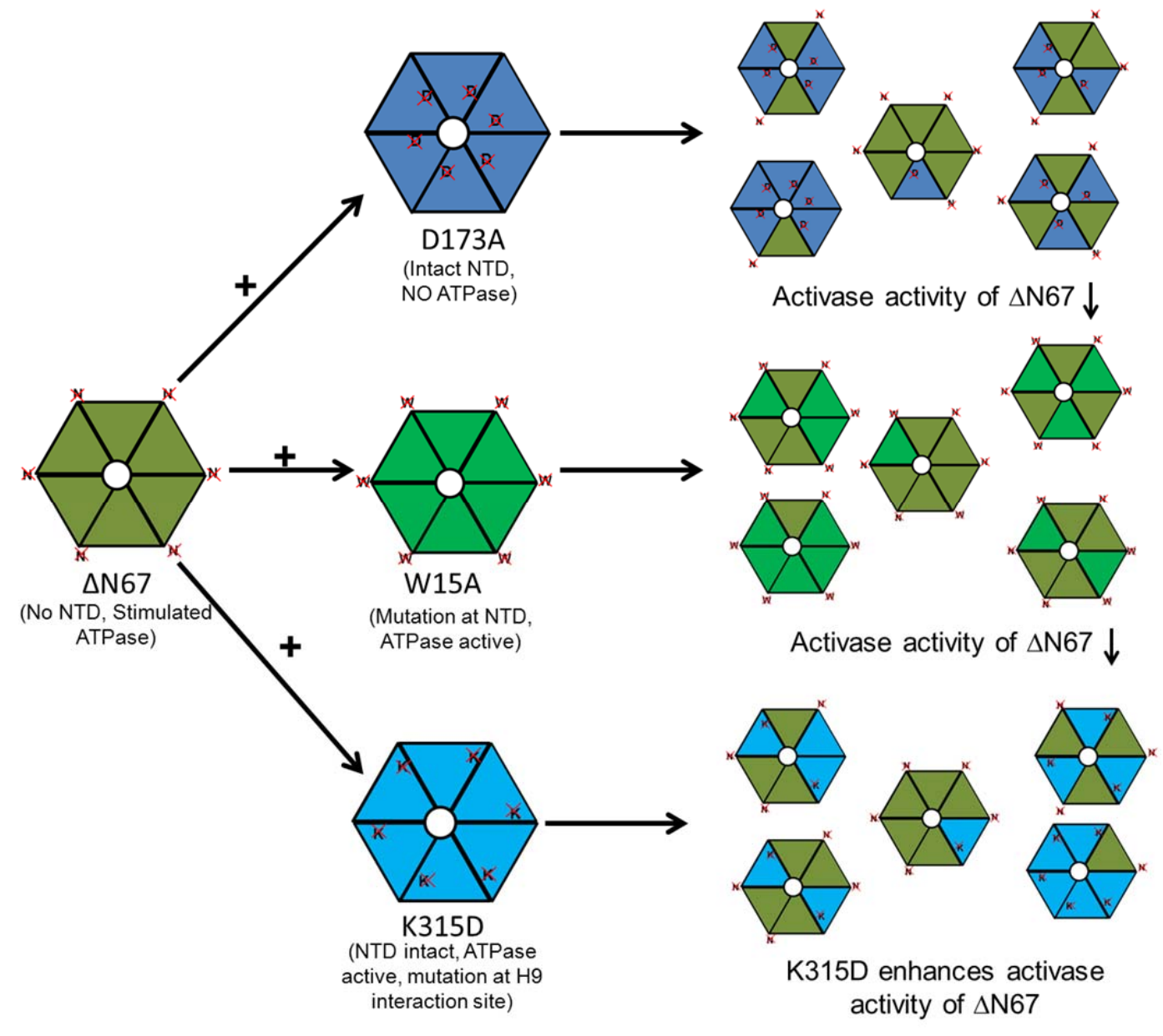

Fig. 5.5: Titration of $\triangle N 67$ Rca with increasing concentrations of different mutants. (A) Surface representation of the hexameric model of tobacco Rca (PDB id: 3ZW6) highlighting the locations of mutated residues. The N-terminal domain is not resolved in the structure. The putative position of the $\mathrm{N}$-terminal is indicated as a dotted circle around the first residue in the model (aspartate 67 in rice). (B) $\Delta$ N67 Rca mutant was pre-incubated with other mutants at 230-250 $\mu \mathrm{M}$ (D173A), 220-230 $\mu \mathrm{M}$ (W15A), 240-290 $\mu \mathrm{M}$ (K315D) Rca protomer concentrations for 10 min to yield a desired proportion. Rubisco activation assay (left) and ATPase assay (right) were performed by maintaining $5 \mu \mathrm{M}$ activase protomer concentration in the final reaction mix at $25{ }^{\circ} \mathrm{C}$. Activase and ATPase activity were calculated considering $\Delta \mathrm{N} 67$ activity as $100 \%$.(C) Experimental scheme with summarized outcomes.

In stark contrast, addition of K315D led to an enhanced activase activity (around 2fold) as compared to $\Delta \mathrm{N} 67$ at low proportions (50\% or less). Increasing the concentration of $\mathrm{K} 315 \mathrm{D}$ beyond $50 \%$ diminishes this stimulatory effect and a drastic reduction in activase activity was observed (Fig. 5.5B). This result could be explained by the fact that this combination contains both the required components for Rubisco remodelling. The necessary $\mathrm{N}$-terminal domain is provided by the helix 9 mutant, while the presence of NTD mutant increases the overall ATPase activity of the 
combined oligomer as compared to $\mathrm{K} 315 \mathrm{D}$ alone (Fig. 5.5B). Although the activase activity of this combination $(\Delta \mathrm{N} 67+\mathrm{K} 315 \mathrm{D})$ was higher than that of the individual components, the overall activity was still a fraction of WT (50-60\%) which does not lack any interaction site. Thus, even if these residues are not solely responsible for activase function, considering the result from Fig. 5.3, 5.4 and 5.5, we may suggest that all residues considered in this study are important for optimal activase functioning to different extents. Functionality of these mixtures indicates that the NTD and the helix 9 interaction site do not have to be localized on the same subunit to function productively.

\subsection{Structure based identification of novel Rubisco interacting residues}

Many AAA+ proteins utilize a partial or complete threading mechanism to remodel the conformation of their binding substrates through the central pore of the hexamer, which features a highly conserved aromatic-hydrophobic-glycine pore-loop 1 motif $[117,125,245]$. This typical conserved motif is absent in the pore loop 1 of plant Rca, although the sequences in the corresponding region are highly conserved in the plant Rca family (Fig. 5.6A). Unfortunately, all pore loops were not resolved in the available structures, presumably due to their flexible nature. As mentioned earlier, the bacterial red-type Rubisco activase CbbX appears to utilizes a classical pore loop threading mechanism [114], but an analogous mechanism for plant activase could not be established [129]. Nevertheless, extensive site-directed mutagenesis of the pore loop segments of the tobacco activase did implicate the pore in its function, possibly to engage larger structural elements of plant Rubisco [129]. As observed previously for NtRca [129], in OsRca the mutation of alanine 143 at the pore loop 1 to valine resulted in a slightly reduced ATPase rate but completely abolished Rubisco activation activity (Fig. 5.6C) suggesting its possible role in substrate remodelling.

The results regarding the pore loop 1 residue $(\mathrm{A} 143 \mathrm{~V})$ indicated that this residue is essential for Rubisco activation. Given that mutating adjacent residues in pore loop 1 did not result in significant phenotypes in the tobacco protein [129], the role of the pore loop 1 in activase function remains to be investigated further. Therefore, we performed a mutational study of the residues further downstream to A143 in rice 
(G144, E145), which had not yet been interrogated. Also, we perused the surface of the hexamer hypothesized to face the Rubisco holoenzyme [246] and identified a number of previously untested, highly conserved surface residues. They were the charged surface residues in helix 3 (K148, Q152, E156, D159) adjacent to pore loop 1 and a protruding surface residue near the specificity helix 9 (R321) (Fig. 5.6A and B).

A
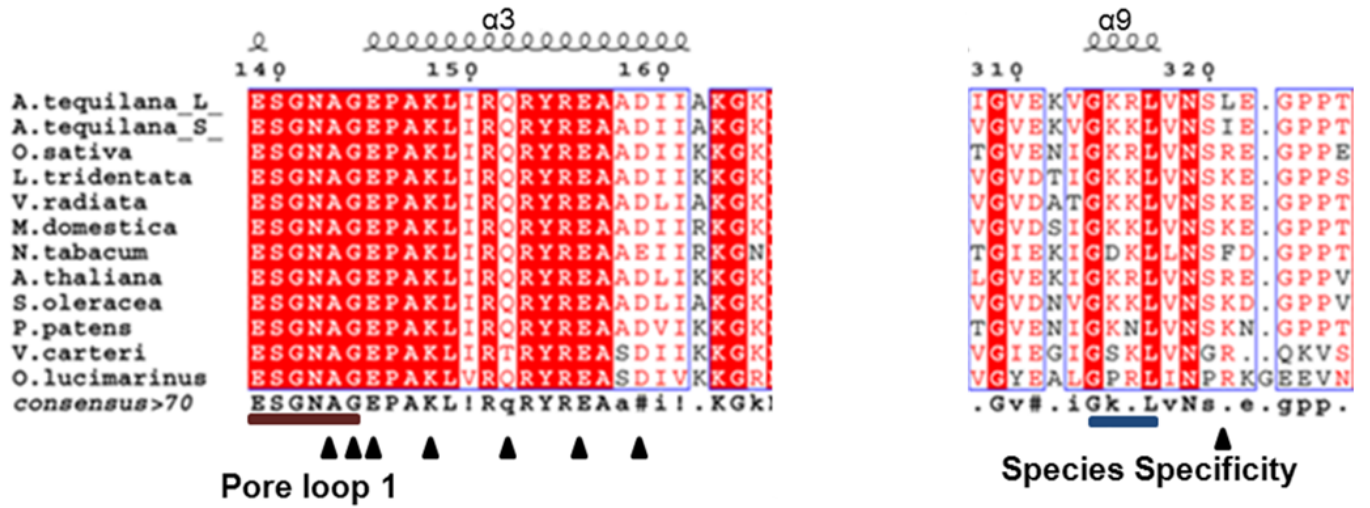

B
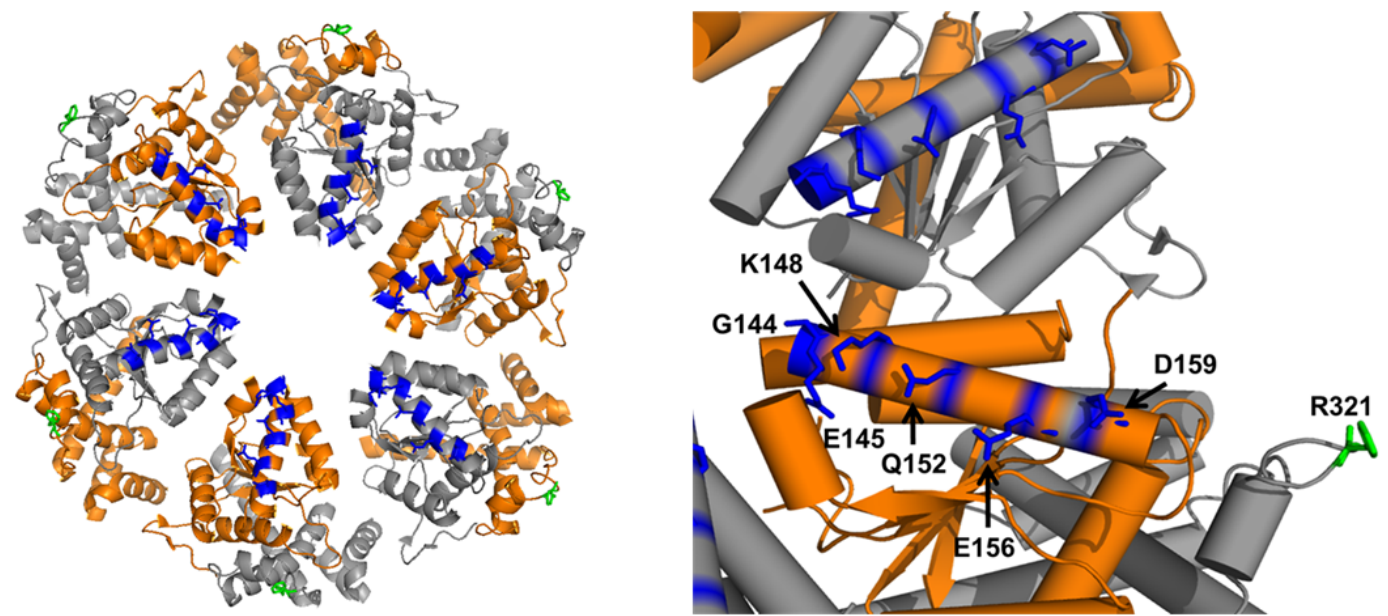


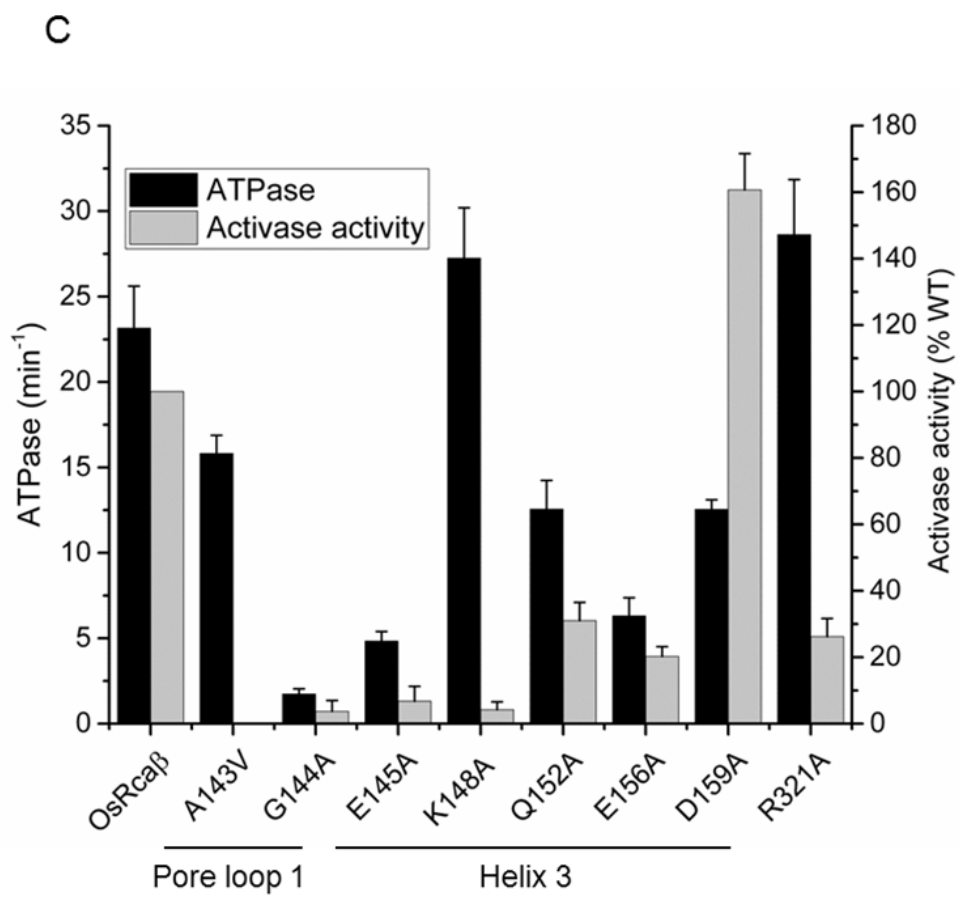

Fig. 5.6: Mutational analysis of pore loop 1, its extension and surface residues. (A) Sequence alignment of activase sequences from different species highlighting the targeted residues for mutation. (B) Ribbon representation of the locations of mutated residues in the hexameric model of tobacco Rca (PDB id: 3ZW6). The position of A143 is not resolved in the structure. (C) ATPase and Rubisco activase activities of WT Rca ( $\beta$ isoform), key pore loop 1 mutant (A143V) extended pore loop 1 mutants (G144A, E145A), surface mutants at helix 3 (K148A, Q152A, E156A, D159A) and protruding surface residue in the structure (R321A), as indicated. Assays were conducted at $25^{\circ} \mathrm{C}$ with $5 \mu \mathrm{M}$ Rca (Protomer), $0.5 \mu \mathrm{M}$ RuBP inhibited Rubisco (ER). Error bar indicates the standard deviations of the mean values of three or more independent experiments.

Mutation in the residues adjacent to alanine 143 (G144A, E145A) led to a $80-90 \%$ loss of ATPase activity with a concomitant $90-95 \%$ reduction of activase function (Fig. 5.6C). Mutational analysis of the surface residues downstream to pore loop 1 gave mixed results. Mutating lysine in this region (K148A) resulted in a slightly enhanced ATPase activity but almost complete loss of activase function. Similarly mutating the surface arginine (R321) near specificity helix resulted in increased ATPase with $\sim 75 \%$ loss of activase function. This implicates these two surface residues in Rubisco interaction. The corresponding residue for arginine in tobacco structure is phenylalanine as shown (Fig. 5.6B). Q152A and E156A resulted in reduction of both ATPase (around 30-50\%) as well Rubisco activation activity (around 20-30\%). Their role is difficult to interpret as the loss of activase function is likely to be the result of low ATPase rate of the mutants. Surprisingly, mutation of an 
aspartate residue in this region (D159A) led to a 40-45\% reduction in ATPase activity but stimulated activase activity ( 1.6 fold), which suggests a role of this residue in Rubisco remodelling. It is possible that either this residue, as a part of surface helix, interacts with Rubisco directly or may be responsible for the conformational changes of the interacting region due to charge differences. The observed behaviour of this mutant could also be the outcome of enhanced affinity for Rubisco and need to be examined using crowding approach in future.

\subsubsection{The key pore loop 1 mutant (A143V) reveals strong subunit coupling with respect to Rubisco activase activity}

Earlier reported data from the literature along with the results discussed in previous section argued for the importance of pore loop1 residue in Rubisco activation [129]. Thus, to further assess the role of the conserved alanine residue, we performed subunit doping experiment between WT and A143V.
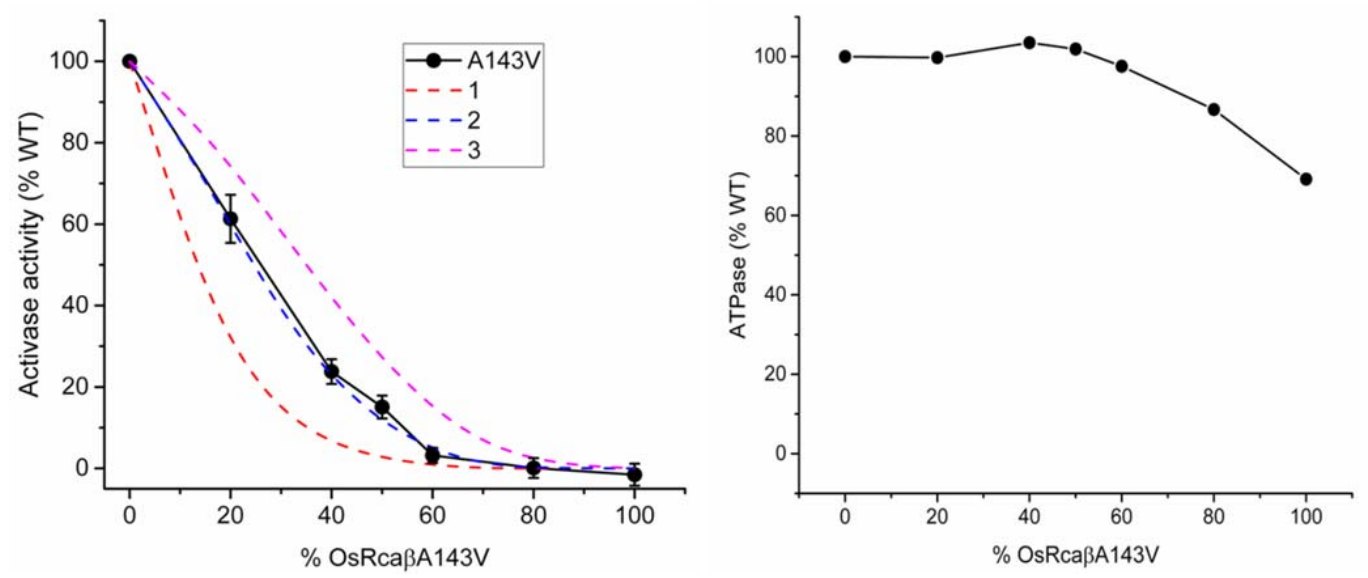

Fig. 5.7: Titration of WT $\beta$-Rca with increasing concentrations of pore loop 1 mutant (A143V). WT active $\beta$ isoform was pre-incubated with the pore loop 1 mutant (A143V) at 220-340 $\mu \mathrm{M}$ concentration range for $10 \mathrm{~min}$ to yield the desired proportion. Rubisco activation assay (left) and ATPase assay (right) were performed by maintaining $5 \mu \mathrm{M}$ activase protomer concentration in the final reaction mix at $25^{\circ} \mathrm{C}$. Activase and ATPase activity was calculated considering WT activity as $100 \%$. Dotted lines indicate the theoretical activity curves if respective number of subunits abolishes hexamer activity. Error bar indicates the standard deviations of the mean values of three or more independent experiments. 
OsRcaßA143V mutant was completely incapable of activating inhibited Rubisco by its own as shown previously (Fig. 5.6C). A sharp drop in activase activity was observed even at a low proportion of pore loop 1 mutant. Loss of $\sim 80 \%$ of maximum activity was observed at an equimolar concentration of WT and mutant (Fig. 5.7). Mathematical analysis of this data using theoretical plots suggests that incorporation of just one or two mutant subunits was sufficient to abolish the activity of the whole hexamer. At the same time, ATPase function was not perturbed (Fig. 5.7). Thus, an Rca hexamer in this case must contain all or at least five active subunits with intact pore loop 1 to remodel Rubisco and subunits are coupled in a globally or sub-globally cooperative manner to remodel Rubisco (Fig. 5.1B and 5.7) [193, 194]. Although, the A143V mutant has a very strong phenotype, the nature of the mutation is unusual, in that a small side chain is replaced with a bulkier one. To functionally impair the pore loop, a bulky aromatic residue like tyrosine is generally mutated [114, 117]. Collectively, these results suggest that pore loop 1 in plant Rca is vital for Rubisco remodelling and has to be intact in a majority of subunits per oligomer for unperturbed Rca function. Furthermore, we have identified key surface residues involved in Rubisco interaction which supports the hypothesized model of RcaRubisco interaction [246]. In future, the important residues found in this study (K148, D159 and R321) must also be analyzed using the mutant doping strategy to further define their role in Rubisco remodelling.

\subsection{Mutational analysis of phophorylating residue at the NTD indicates its role in Rubisco-Rca interaction and might be an alternate regulatory mechanism in plants under light dark transition}

Protein phosphorylation is an important type of post-translational modification which can modulate the activity of an enzyme or protein. Effect of protein phosphorylation has been studied for many AAA+ proteins such as the mitotic factor p60 and p97 [247-249] leading to inhibition of their activity. Recent phosphoproteomics studies have found Rca to be an abundantly phophorylated protein in photosynthetic tissues. Furthermore, it has been predicted that Rca activity could also be modulated by phosphorylation at a site critical for its activity like ATPase rate or its interaction with Rubisco $[250,251]$. Thus, protein phosphorylation could be another regulatory 
mechanism in plants in addition to redox regulation under light/dark cycle. However, recently redox changes in chloroplast stroma have been reported as the major trigger behind the phosphorylation of Rca, possibly due to oxidation of thioredoxins [252]. It was also suggested that phosphorylation may not be responsible for downregulating in vivo Rca activity in arabidopsis at least in the presence of redox regulation. Nevertheless, it was predicted that phosphorylation might regulate Rca activity when redox regulation is not operating or under other stress related conditions [252].

Rca in arabidopsis was found to be phosphorylated at threonine 78 and serine 172 (numbering includes transit peptide), which are located at the NTD and the Walker A region of Rca respectively $[250,253]$. However, only threonine 78 was responsive to dark/light response and found to be more phosphorylated in the dark, while serine 172 remained unmodulated. Thus, it was predicted that phosphorylation of threonine 78 could be associated with inhibition of Rca activity in the dark when the CalvinBenson cycle is inactive [250]. Since this residue is located at the NTD, it is conceivable that this modification modulates the Rubisco activation state by preventing the Rubisco-Rca interaction. However, this threonine 78 is not conserved and replaced by isoleucine in rice and maize. Also, no phosphorylation was found for Rca in maize but Rca was found to be phosphorylated at serine (S23 after removing transit peptide) adjacent to isoleucine in rice chloroplasts [251].

To study this phenomenon, we produced two mutants at this position, S23A and S23D. Substituting serine with alanine, tests for the function of hydroxyl group at this position. Aspartic acid is a commonly used phosphomimetic substitution similar to phosphoserine. Both variants were found to be fully ATPase active and retained the original activity of WT but lost almost $70-80 \%$ activase function (Fig. 5.8). Since NTD is responsible for Rca-Rubisco interaction and not for ATPase activity, it makes sense that the mutants retained full ATPase activity as observed for earlier NTD mutants such as W15A and $\Delta \mathrm{N} 67$ (section 5.2). It also indicates that the oligomeric structure of these Rca variants is not compromised. The results showed that serine 23 is likely involved in the Rca-Rubisco interaction. We believe protein phosphorylation could be a good target for studying Rubisco regulation in plants in future. Most of the aspects of protein phosphorylation pertaining to Rca are not well characterised. The nature of the kinase (plastid casein kinase 2) performing phosphorylating function has 
recently been reported [252] and could be a promising candidate for regulation and mutational study.

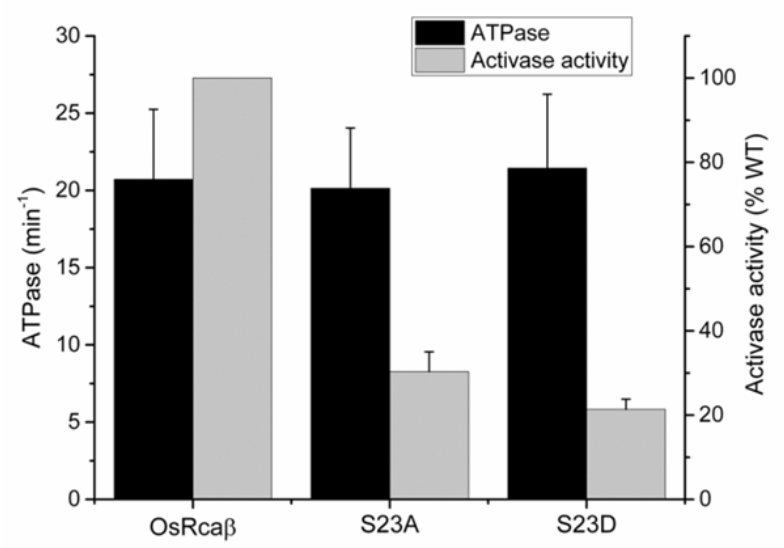

Fig. 5.8: Mutation of the phosphorylated residue at the N-terminal domain of rice Rca. ATPase and Rubisco activase activities of S23A and S23D variants along with WT Rca ( $\beta$ isoform). Assays were conducted at $25^{\circ} \mathrm{C}$ with $5 \mu \mathrm{M}$ Rca (Protomer). Error bars indicate the standard deviations of the mean values of three or more independent experiments.

\subsection{Mutation of conserved arginine (R293V) at the Rea subunit interface ablates its ability to exchange subunits}

It has been shown using tobacco activase that mutating the conserved arginine-294 (in tobacco) to valine or alanine resulted into formation of stable hexamer in the presence of ATP $\gamma$ S using techniques such as nanoESI mass spectrometry [140], negative stain electron microscopy [129] and analytical ultracentrifugation/SAXS [141]. Earlier mutagenesis study of this residue showed that mutating arginine 294 to alanine in tobacco led to a reduced ATPase rate and Rubisco activation, while mutating the same arginine to valine resulted in a WT-like phenotype $[129,165]$. This suggests that this substitution has locked the enzyme into a functional hexameric active form which should be an ideal candidate for structural studies.

Therefore, we targeted the corresponding residue in rice (R293) for mutagenesis, hoping to obtain a stable hexamer for rice Rca for both $\alpha$ and $\beta$ isoform. This mutant could have been utilised for comparative structural studies between rice and tobacco and for determining the overall structure of $\alpha$-Rca along with its C-tail extension, since tobacco only possesses the $\beta$ isoform. However, mutation of arginine to valine 
(R293V) in rice resulted in a completely non-functional phenotype devoid of ATPase as well Rubisco activation activity (Fig. 5.9A). This suggests that Rca in these two plant species could differ in intermolecular contacts due to structural variation in this region, which also led to different oligomerization pattern in the two species.

Analytical gel filtration chromatography of this variant indicated an elution pattern corresponding to the void volume of the column which was concentration independent (Fig. 5.9B). This indicates that either this variant might have formed soluble aggregates or acquired a spiral high molecular weight oligomeric form, which is concentration and nucleotide independent in rice. The secondary structural elements of this variant should be analyzed using $C D$ and if found intact, this variant would be a promising candidate for structural studies using cryo-electron microscopy. It was also observed that this mutant had lost its ability to hetero-oligomerize and exchange subunits with other Rca. The mixing experiment between WT Rca and R293V subunits did not result in subunit poisoning in terms of ATPase activity in our experiments (Fig. 5.9C).

A

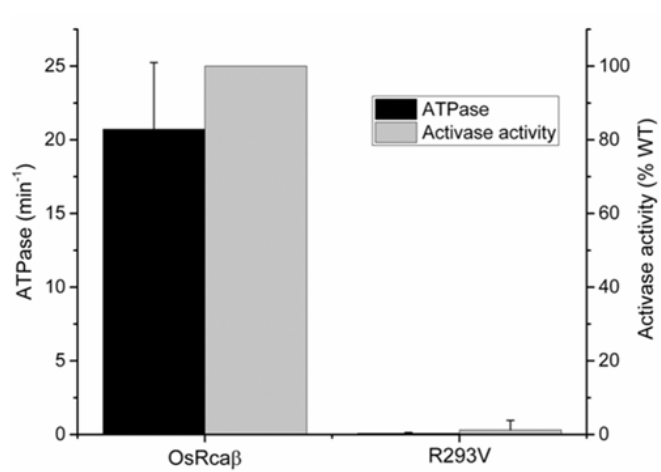

B

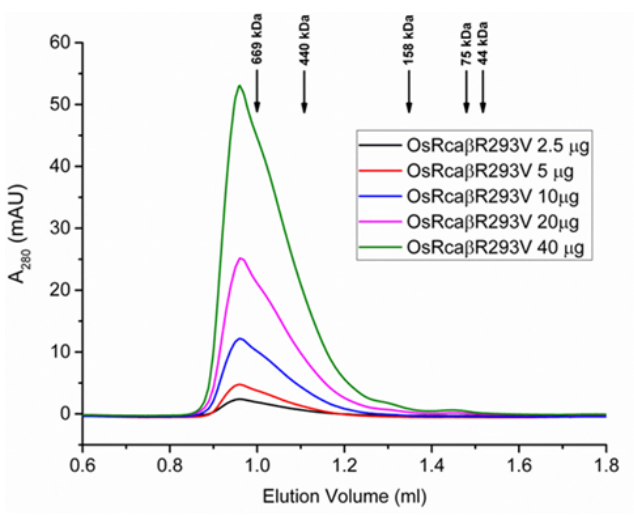

C

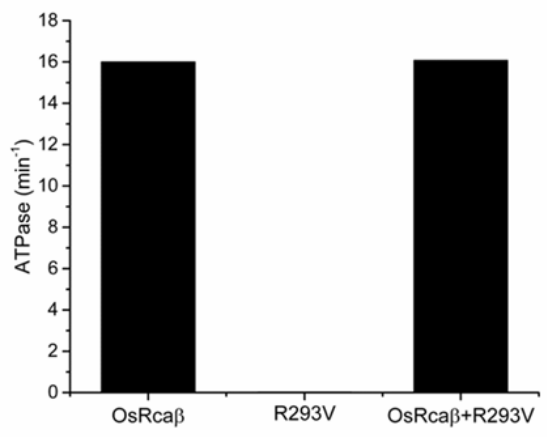


Fig. 5.9: Mutation of conserved arginine at Rca interface: (A) ATPase and Rubisco activase activities of conserved arginine at Rca interface (R293V) along with WT Rca ( $\beta$ isoform). Assays were performed at $25^{\circ} \mathrm{C}$ with $5 \mu \mathrm{M}$ Rca (Protomer). Error bars indicate the standard deviations of the mean values of three or more independent experiments. (B) Analytical gel filtration chromatography of OsRcaßR293V at different concentration performed using a Superdex 200 3.2/30 in $20 \mathrm{mM}$ Tris- $\mathrm{HCl}$ pH 8.0, $50 \mathrm{mM} \mathrm{NaCl}, 5 \mathrm{mM} \mathrm{MgCl} 2,0.5 \mathrm{mM}$ ATP. (C) Mixing experiment between OsRca $\beta$ and Rca interface mutant $(\mathrm{R} 293 \mathrm{~V})$ at an equimolar concentration in the final reaction mix $(5 \mu \mathrm{M}$ each). The two proteins (170 $\mu \mathrm{M}$ Rca protomer) were pre-incubated for $10 \mathrm{~min}$ prior to assay.

In this chapter we have carried out an extensive study of activase variant to validate the mutational phenotypes of various previously reported residues. Moreover, by applying a subunit doping strategy, we generated additional information about the relative contribution of the targeted residues to Rubisco interaction and remodelling. Furthermore, several novel structurally guided mutants were generated and biochemically characterized to add to our appreciation of activase function. Based on our observations, we have left many open questions, while generating a molecular toolbox to address them. In future, our library of mutants will allow the design of additional experiments that will permit inferences to be made about the precise mechanism of Rubisco activation and other functional aspects of the Rca enzyme. 


\section{A preliminary characterization of the putative Rubisco activase isoform (Rca2) of rice}


In chapter 3, we provided a characterization of the two abundant Rca isoforms found in rice. However, genomic studies suggest that these may not represent the entire Rubisco activation machinery in rice or plants in general. In particular, an uncharacterized putative Rubisco activase isoform sometimes designated as Rubisco activase 2 or Rca2 has been found to be encoded by the nuclear genome of almost all plants and algae [8].

The homologue of this protein in arabidopsis (uniprot id: Q9AST9) has been detected as a stromal chloroplast protein in many proteomics experiments [254](Plant Proteome Database, Cornell) [255]. The gene is mainly found to be transcribed in photosynthetic tissue, which seems consistent with its possible role in Rubisco activation. However, its expression level is much lower than that of the orthodox activase (appendix Fig. 1A and B) (arabidopsis eFP browser) [256]. The Rca2 (gene id: Atlg73110) shows negligible or almost no expression in non-photosynthetic tissues such as root. Peak expression levels were found in the mesophyll and guard cells after water spray. Under stress conditions such as ABA treatment, the expression level in these photosynthetic tissues was significantly reduced. The patterns of change in the expression levels were similar to the activase under identical conditions however, the expression signal intensity was much less for Rca2 (appendix Fig. 1A and B). This supports a possible functional association between Rca2 and Rca as genes with similar sequence and expression profiles are likely to exhibit a functional relationship [257, 258].

From the phylogenetic tree analysis (17 angiosperm, 1 bryophyte and 4 green algae) it is apparent that Rca2 and Rca diverged prior to the evolution of land plants, probably following a gene duplication event in the green algae (Fig. 6.1). The complete conservation of both isoforms in all examined higher plant and green algal genomes suggests that Rca2 evolved to play an important, albeit unknown role. The tree illustrates that Rca isoforms are in general more highly conserved than Rca2 isoforms, indicating that a more stringent selection pressure is acting on the former, canonical Rubisco activase sequences. 


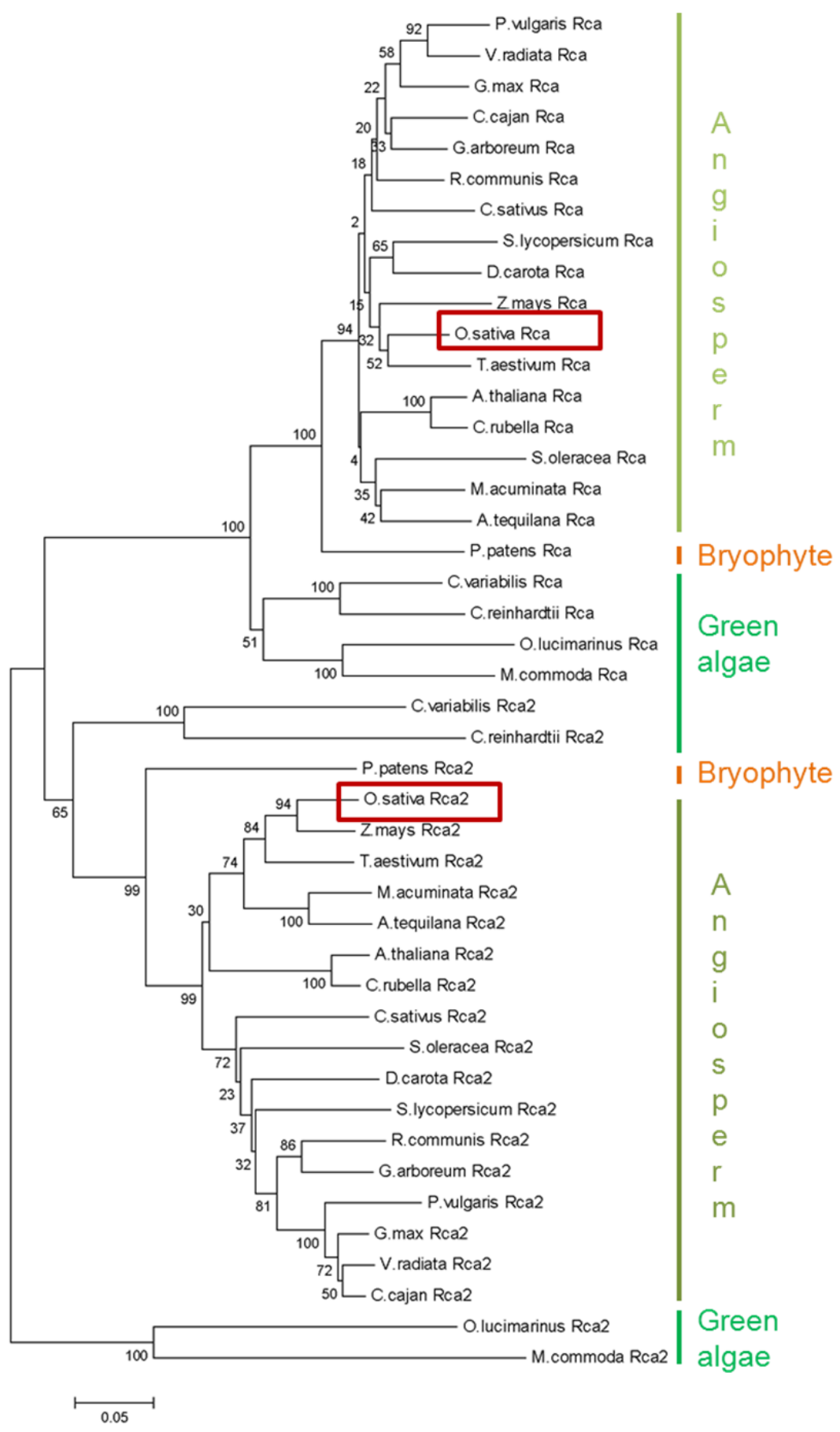

Fig. 6.1: Phylogenetic tree of plant and algal activase and putative Rca2 sequences. The sequences from seventeen higher plants, one bryophyte, and four green algae (appendix Table 1) were used to generate a phylogenetic tree by Neighbour joining using Mega 6 software [259] using the P-distance model and pair wise gap deletion. Bootstrap values are the percentage from 500 replicates where the given node was present. 
From a functional perspective, a sequence alignment of Rca2 homologue in rice with the Rubisco activase predicts intact ATPase active site motifs (Walker A and B motifs are intact) along with a conserved arginine finger (R243 in OsRca). However, the known protein-protein interaction site (specificity helix, $\alpha 9$ ) with Rubisco is not conserved (Fig. 6.2). Further comparative analysis of the sequences from different Rca2 and activase homologues in plants revealed conservation among some additional residues responsible for maintaining ATPase function (Fig. 6.2). Most of the residues shown to be important for activase activity were not conserved except the tryptophan (W15 in rice activase) at the N-terminal domain (NTD) and the alanine (A143) at pore loop 1. Also, the low sequence identity (48\%) between Rca and Rca2 (residues 19395 in the alignment for OsRca2) in rice suggests that Rca2 might not be a functional activase by itself. Nevertheless, the residues predicted to be important for subunitsubunit interactions in activase [129] were found to be conserved between Rca2 and Rca. Therefore, we hypothesized that Rca2 might somehow modulate the activity or function of Rca by forming a hetero-oligomeric complex with it. Interestingly, few regions at the NTD are distinctly conserved in Rca2. This suggests that the NTD of Rca2 may be involved in specific functions such as substrate anchoring as predicted for the NTD of Rca [59, 129].

In this section, we aimed to characterize this Rca2 isoform to elucidate its biochemical function. We were able to purify a truncated form of the protein using the heterologous E.coli expression system. Our initial results indicate that Rca2 can oligomerize with OsRca $\beta$ to form an ATPase functional complex. These results indicate that there is much more to be learnt about the complexity of plant Rca function. 


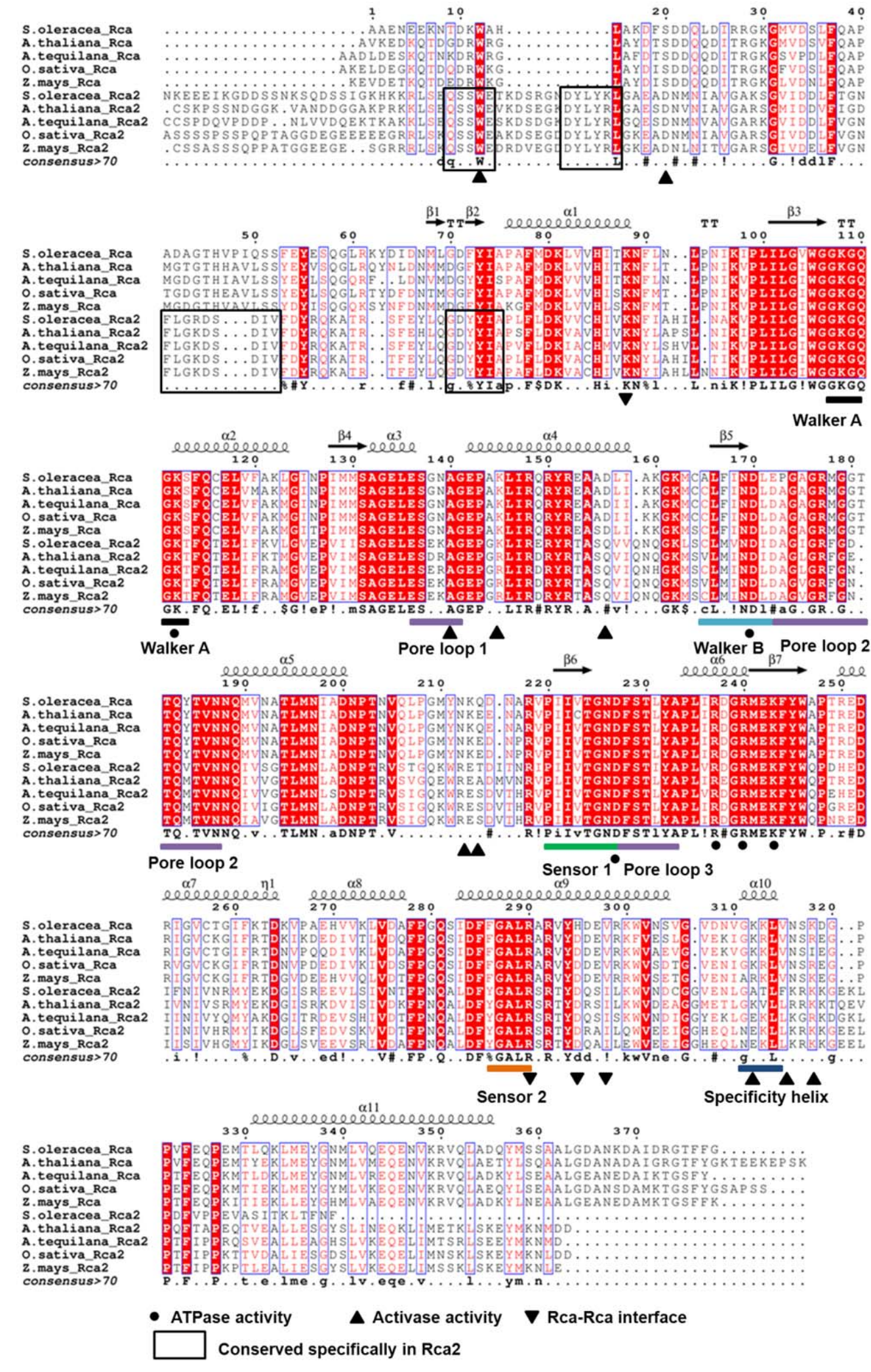


Fig. 6.2: Protein sequence alignment between plant Rca and Rca2 isoforms: Amino acid sequences of different Rubisco activase and putative Rca2 isoforms were aligned using ESPript [184] after removing the transit peptide as predicted by the ChloroP online server. The ATPase active sites in $\mathrm{AAA}+$ domain (Walker A and Walker B) were conserved between the two groups of proteins including other residues responsible for ATPase function. The interaction site with Rubisco (lysine 315 in OsRca $\beta$ ) was not conserved as marked in the alignment along with other interaction sites including the ones discovered in the present study. The Rca-Rca interface residues were also found to be conserved between Rca2 and Rca in plant. The N-terminal domain of contained some distinct region which are conserved in Rca2 but not in Rca. The uniprot and NCBI accession code of the proteins utilized for creating the sequence alignment has been tabulated in the appendix Table 1.

\subsection{Purification of OsRca2}

The gene encoding Rca2 of Oryza sativa (Uniprot accession code: Q7XN85, expressed amino acid 47-441 (after removing transit peptide using the ChloroP online server) was synthesized by GenScript (USA). The Rca2 was heterologously expressed in E.coli BL21 as a N-terminal fusion with $\mathrm{His}_{6} \mathrm{Ub}$ and purified exactly as Rca (section 3.1.1). However, the expressed protein was completely insoluble even after several attempts. The protein parameters analysis of full length Rca2 using ProtParam tool [186] revealed the protein as unstable based on the computed instability index parameter. Thus, we analyzed a sequential deletion of the $\mathrm{N}$-terminal residues and found that after removing the first 21 amino acids the instability index was within a stable range.

The corresponding construct named pHue $\Delta \mathrm{N} 21 \mathrm{OsRca} 2$ was cloned and the protein was purified using identical purification steps to other activases (section 3.1.1). Now the protein was found be soluble upon expression and could be easily purified using affinity chromatography and size exclusion chromatography (Fig. 6.3).

Initially, we suspected that probably these deleted residues at the $\mathrm{N}$-terminal region of Rca2 constitute a membrane anchor considering association of Rca2 with thylakoid membrane in the proteomics experiments [254]. Removing them would thus have led to solubilization of protein. However, analysis of the sequence using the TMHMM online server did not predict any transmembrane helices in the given region. Another possibility could be the erroneous prediction of the chloroplast transit peptide by the ChloroP server. 
A

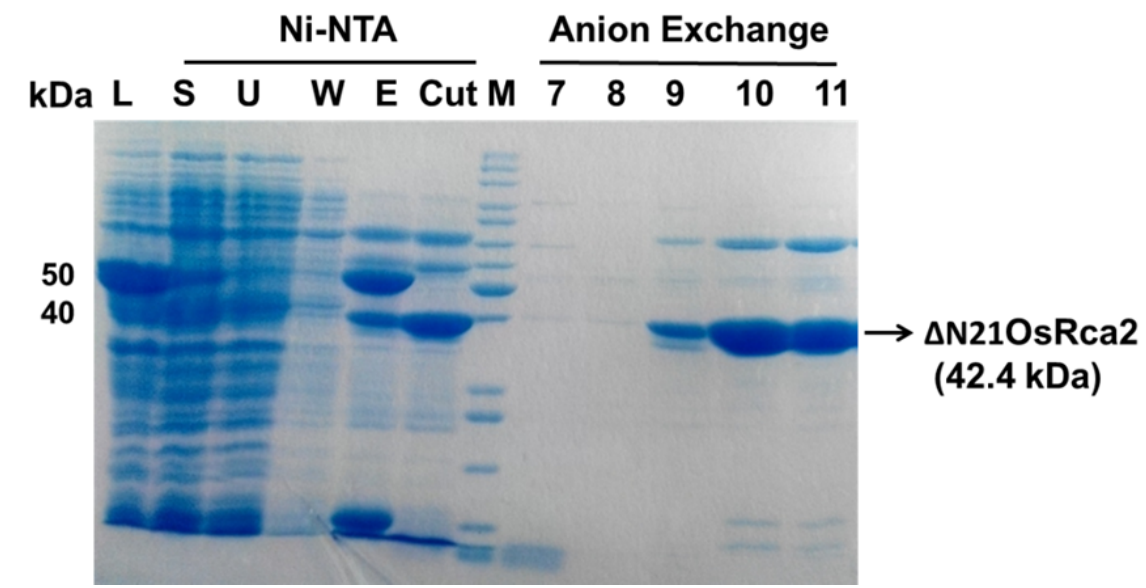

B
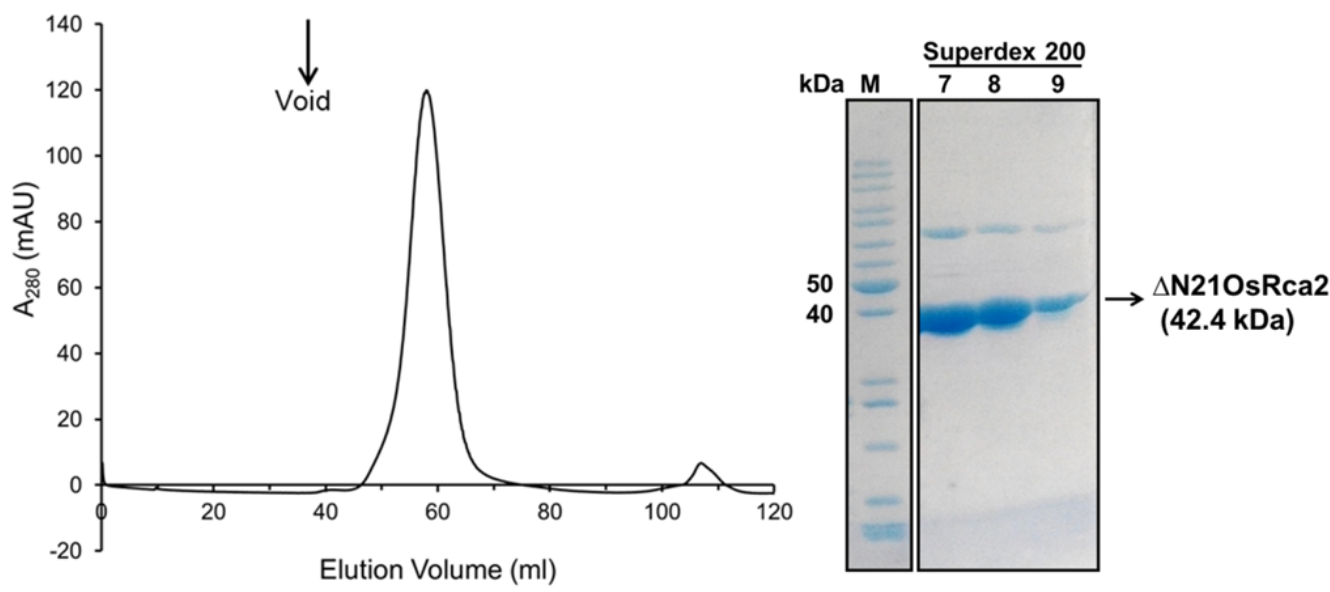

Fig. 6.3: Representative purification of $\Delta \mathbf{N 2 1 O s R c a 2 . ~ ( A ) ~} 12.5 \%$ SDS-PAGE analysis. Successive purification by IMAC (Ni-NTA), followed by Usp2 mediated cleavage of N-terminal tag and anion exchange chromatography (AIEC) yielded pure protein. (M: Molecular Weight Marker, L:Lysate, S:Soluble, Ni-NTA: Fractions after IMAC, U: Unbound, W:Wash,: E: Final eluate, Cut : eluate after USP2 mediated cleavage of Ubiquitin. (B) Size exclusion chromatogram of $\Delta$ N21OsRca2 showed a single peak of purified protein (left). Purified fractions from gel filtration column on a $12.5 \%$ SDSPAGE gel (right). 


\section{2 $\Delta$ N21OsRca2 migrates as a monodisperse species by gel filtration and native PAGE}

In order to assess the oligomeric assembly and elution pattern of the Rca2 at different concentration, we performed an analytical gel filtration analysis of $\Delta \mathrm{N} 21 \mathrm{OsRca} 2$ at different concentrations followed by native PAGE. We observed that the protein was eluted mostly as a single peak with a minor shoulder at all tested concentrations (Fig. 6.4A), which was further confirmed by native PAGE of the samples (Fig. 6.3B). $\Delta \mathrm{N} 21$ OsRca2 dominantly displayed monodispersity with the major peak eluted at $1.23 \mathrm{ml}$, which corresponds to a Stokes radius consistent with a AAA+ hexamer as recently reported by our group for the red-type activase from red algae [104]. These results were highly distinct to the canonical activase, which shows high polydispersity with a concentration-dependent size as described earlier (section 3.5.2). Therefore, the monodispersity of Rca2 makes it a promising candidate for structural studies in future. Although the majority of the protein eluted as a hexameric species, we also observed some smaller bands on native PAGE (Fig. 6.3B), which could be the dissociated oligomers as suggested by their cross-reaction to an anti-Rca antibody (Fig 6.9). These results suggest that the oligomeric assembly of Rca2 is intact after the $\mathrm{N}$-terminal truncation and this protein could be utilized for biochemical study.

A

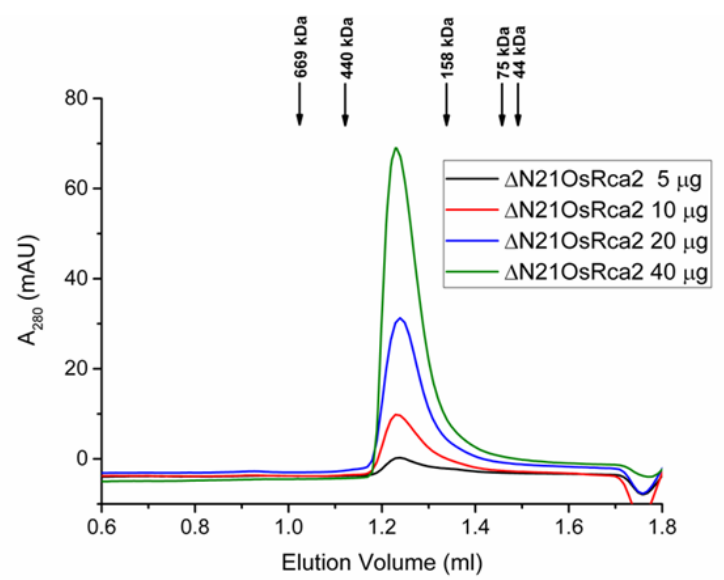


B
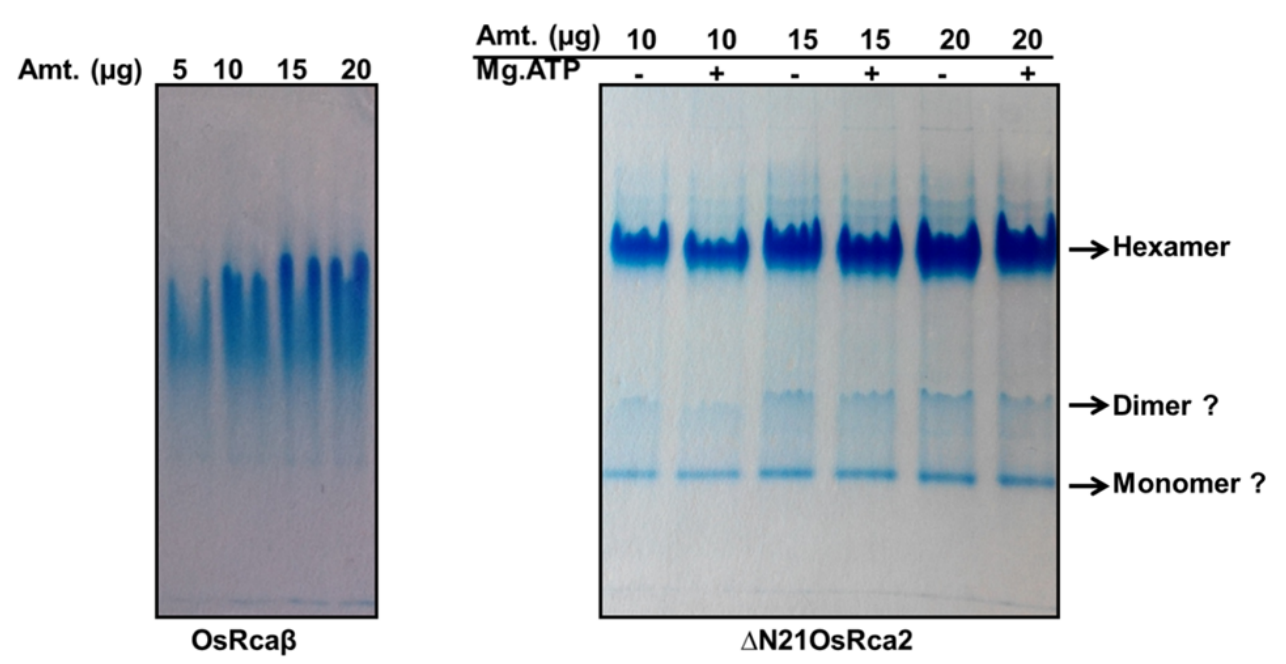

Fig. 6.4: Rea2 eluted as monodispersed species as analyzed by analytical gel filtration chromatography and native PAGE. (A) Analytical gel filtration chromatography of $\Delta \mathrm{N} 21 \mathrm{OsRca} 2$ at different concentrations performed using a Superdex $2003.2 / 30$ in $20 \mathrm{mM}$ Tris- $\mathrm{HCl} \mathrm{pH} 8.0,50 \mathrm{mM}$ $\mathrm{NaCl}, 5 \mathrm{mM} \mathrm{MgCl} 2,0.5 \mathrm{mM}$ ATP. (B) $6 \%$ Native PAGE analysis of $\Delta \mathrm{N} 21$ OsRca2 (right) compared to OsRca $\beta$ (left) at different concentrations. Amount of protein loaded has been indicated against each lane.

\section{3 $\Delta$ N21OsRca2 was ATPase inactive and unable to activate rice Rubisco but forms ATPase functional hetero-oligomers with OsRca $\beta$}

Biochemical characterization of the purified $\Delta \mathrm{N} 21$ OsRca2 revealed it to be nonfunctional in terms of ATPase as well as Rubisco activation (Fig. 6.5). However, as per our hypothesis, Rca2 may not be a functional activase by its own. Therefore, we performed subunit mixing experiments between $\Delta \mathrm{N} 21$ OsRca2 and OsRca $\beta$ to determine whether Rca2 can modulate the activity of Rca $\beta$ in terms of its ATPase or activase function. We observed no significant effect of Rca2 mixing on Rca $\beta$ when analyzed using ATPase and Rubisco activation assay (Fig. 6.5). Generally, ATPase inactive subunits poison the activity of functional subunits as demonstrated previously (section 3.5.1 and 5.1). Contrary to this, Rca2 did not poison the activity of Rca $\beta$, which indicates that Rca $\beta$ can tolerate the presence of Rca2 without being inhibited. Therefore, we again hypothesized that Rca2 may have some modulatory effect on Rca $\beta$ or form an functional active site only in presence of Rca $\beta$ but these small effects might not be detectable given the high activity of wild-type Rca $\beta$. 

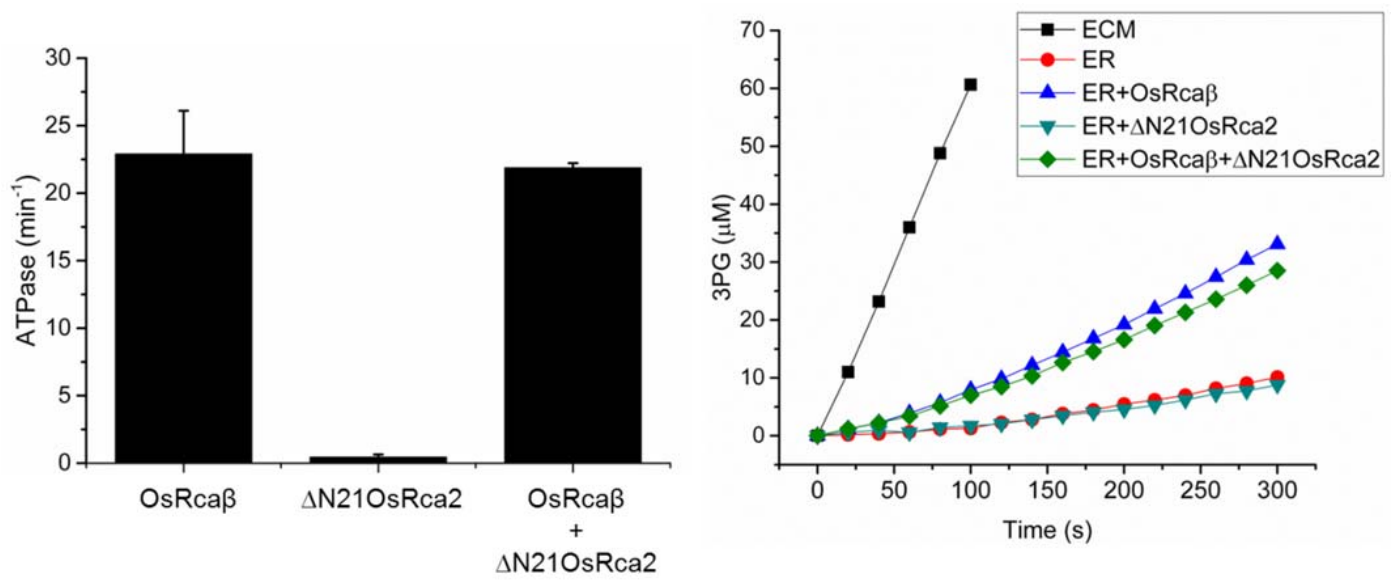

Fig. 6.5: Biochemical characterization of $\Delta \mathbf{N 2 1 O s R c a 2 . ~ ( A ) ~ A T P a s e ~ ( l e f t ) ~ a n d ~ R u b i s c o ~ a c t i v a t i o n ~}$ (right) assay of $\Delta \mathrm{N} 21 \mathrm{OsRca} 2$ along with control WT Rca ( $\beta$ isoform). Assays were performed at $25^{\circ} \mathrm{C}$ with $5 \mu \mathrm{M}$ Rca and $\Delta \mathrm{N} 21$ OsRca2 (Protomer). For mixing experiments two proteins (around $190 \mu \mathrm{M}$ together) were pre-incubated for $10 \mathrm{~min}$ to yield an equimolar concentration of $5 \mu \mathrm{M}$ each in the final reaction. ATPase activity of the mix sample was calculated in terms of functional Rca $\beta$ active site.

To resolve this issue and further test our hypothesis, we replicated the subunit mixing experiments using the ATPase inactive Walker B mutant (OsRcaßD173A). This experiment examined if Rca2 can form a functional hetero-oligomer with OsRcaßD173A. Thus, systematic subunit mixing experiments were performed with $\Delta \mathrm{N} 21$ OsRca2 and OsRcaßD173A by keeping the concentration of one as constant at $10 \mu \mathrm{M}$ in final reaction mix, while titrating up the concentration of other and viceversa (Fig. 6.6A). As hypothesized, we discovered that mixing the non-functional $\triangle \mathrm{N} 21$ OsRca2 and OsRcaßD173A resulted in detection of ATPase hydrolysis (Fig. 6.6A). ATP hydrolysis rate was dependent on the concentration of Rca2. Almost constant ATPase was observed, when Rca2 was kept constant and OsRcaßD173A was varied (Fig. 6.6A). However, the observed ATPase activity was relatively low and did not increase proportionally with the concentration of OsRca2. Therefore, data have been presented additionally in terms of ATPase activity per minute for total molar concentration of the OsRca2 present in the reaction as well as specific ATPase activity (Fig. 6.6A and B).

Ten $\mu \mathrm{M}$ of D173A possessed a basal ATPase activity of around $3.3 \mu \mathrm{M}$ ATP $\min ^{-1}$ $\left(0.33 \mathrm{~min}^{-1}\right)$, which increased to $14.4 \mu \mathrm{M} \mathrm{ATP} \min ^{-1}\left(1.44 \mathrm{~min}^{-1}\right)$, when mixed with an equimolar concentration of Rca2, which possessed a basal ATPase activity of 4.8 $\mu \mathrm{M}$ ATP $\min ^{-1}\left(0.48 \mathrm{~min}^{-1}\right)$. The basal activities of the individual isoforms could be the outcome of contaminant ATPases and their values were below the sensitivity of 
the method. When the Rca2 to OsRcaßD173A ratio was reduced to below 1 (Rca2: D173A), the ATPase activity was also reduced and minimum activity was detected at $2 \mu \mathrm{M}$ of Rca2 $\left(4.77 \mu \mathrm{M}\right.$ ATP $\left.\min ^{-1}\right)$, while maximum at $20 \mu \mathrm{M}$ Rca2 concentration (18.7 $\mu \mathrm{M}$ ATP $\mathrm{min}^{-1}$ ) (Fig. 6.6A). Contrary to this, when the concentration of Rca2 was kept constant at $10 \mu \mathrm{M}$ and concentration of OsRcaßD173A was varied, the ATPase activity almost reached its maximum value at $2 \mu \mathrm{M}$ Rca2 (12.26 $\mu \mathrm{M}$ ATP $\left.\min ^{-1}\right)$. Titrating up the concentration of OsRcaßD173A further even up to $20 \mu \mathrm{M}$ in this case did not increase the ATPase activity of the oligomeric mixture (Fig. 6.6A). We noticed that on one hand the ATPase activity of the hetero-oligomer kept increasing by titrating up the concentration of Rca2, whereas the ATPase activity per OsRca2 active site was reduced during the titration (Fig. 6.6A and B). This indicates that a small number of OsRca2 subunits are capable to restore functionality and more subunits led to a reduction in ATPase activity per active site. There is therefore an optimal ratio of OsRca2:OsaRcaßD173A subunits, and increasing the concentration of OsRca2 leads to less functional assemblies.

A
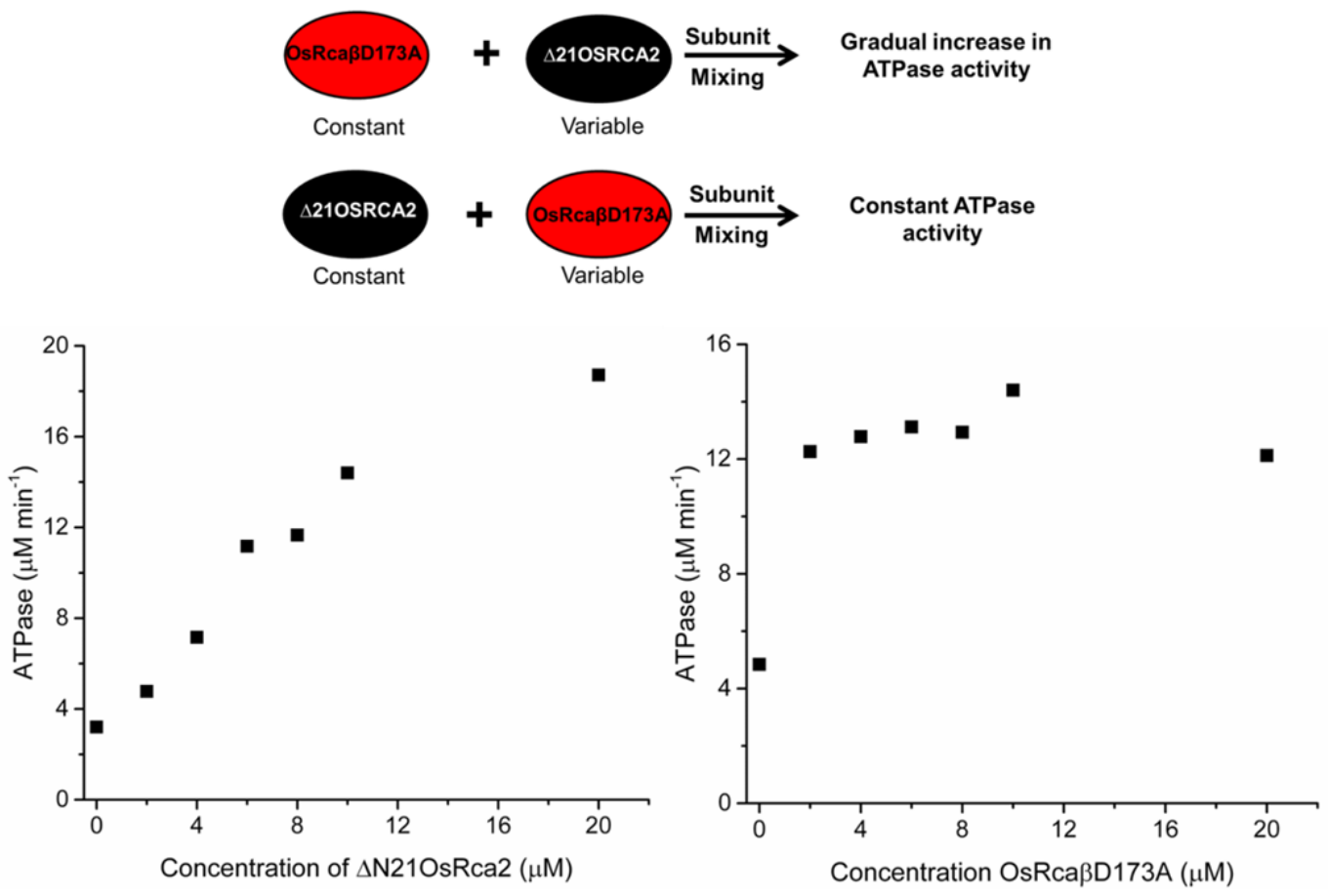
B
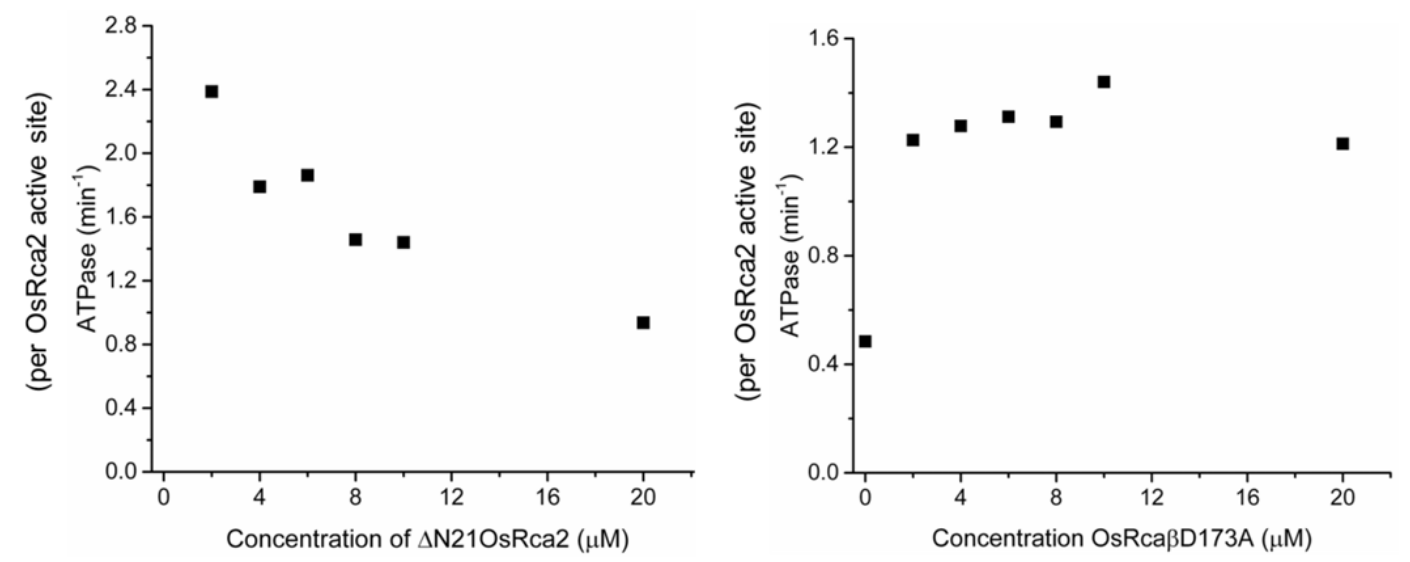

C

D
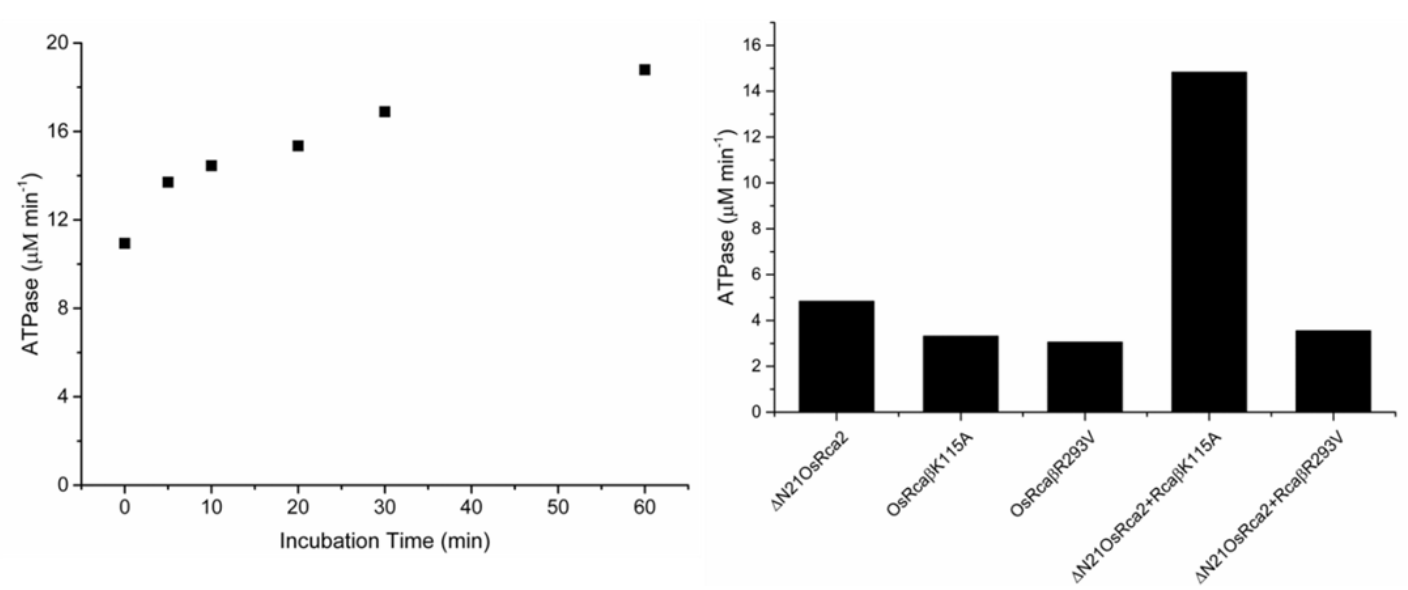

E

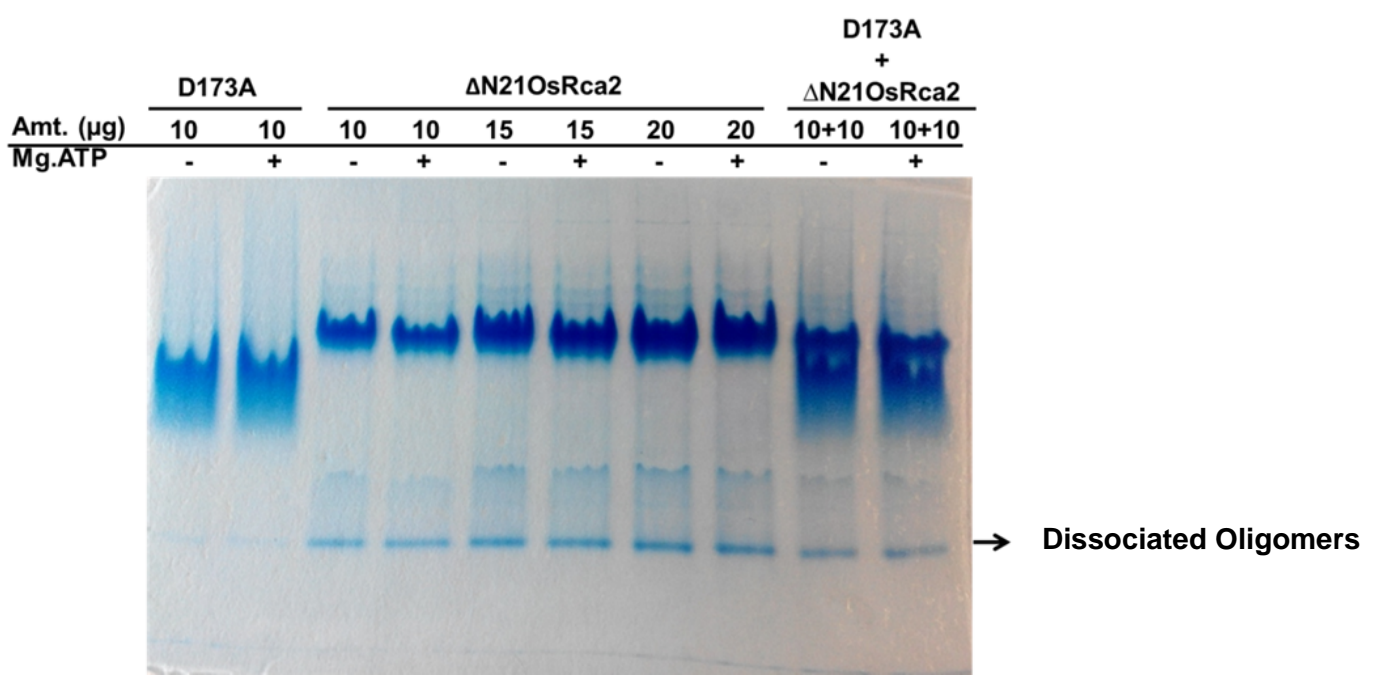


Fig. 6.6: Biochemical characterization of OsRca2. (A) Subunit mixing experiments between Walker B mutant of Rca $\beta$ (D173A) and $\Delta \mathrm{N} 21$ OsRca2 were performed such that in one experiment concentration of OsRca $\beta D 173 \mathrm{~A}$ was kept constant $(10 \mu \mathrm{M})$ in the final reaction mix and $\Delta \mathrm{N} 21$ OsRca2 was varied (left) while in another experiment the conditions were reversed (constant $\Delta \mathrm{N} 21 \mathrm{OsRca} 2$ and varied OsRca $\beta \mathrm{D} 173 \mathrm{~A}$ ) (right). (B) Representation of the data from A as activity per $\Delta \mathrm{N} 21 \mathrm{OsRca} 2$ active site. The two proteins were pre-incubated at 160-220 $\mu \mathrm{M}$ concentration range (protomer) for 10 min. (C) The subunit mixing experiment at an equimolar concentration of OsRcaßD173A and $\Delta \mathrm{N} 21$ OsRca2 $(10 \mu \mathrm{M}$ each in final reaction mix) with varying incubation time. (D) The subunit mixing experiment of $\Delta \mathrm{N} 21$ OsRca2 with OsRca $\beta \mathrm{K} 115 \mathrm{~A}$ and OsRca $\beta \mathrm{R} 293 \mathrm{~V}$ at an equimolar concentration (10 $\mu \mathrm{M}$ each in final reaction mix) along with control rates for $10 \mu \mathrm{M}$ protomer. ATPase assay were performed at $25{ }^{\circ} \mathrm{C}$. (E) $6 \%$ Native-PAGE gel of OsRcaßD173A and $\triangle 21$ NOsRca2 alone and after mixing. Loaded amounts are indicated against each lane.

This study also revealed that subunit mixing in this case was very slow and ATPase activity of the hetero-oligomeric complex kept increasing with incubation time (Fig. 6.6C). For instance, ATPase activity of $\sim 10.9 \mu \mathrm{M} \mathrm{ATP} \min ^{-1}$ was observed at an equimolar concentration (10 $\mu \mathrm{M}$ each) without any pre-incubation. After 10 minutes of incubation time, observed ATPase was $14.4 \mu \mathrm{M}$ ATP $\min ^{-1}$, which further increased to $18.7 \mu \mathrm{M}$ ATP $\min ^{-1}$ after 60 minutes of incubation (Fig. 6.6C). However, even under subunit mixing condition, no Rubisco activation was observed for the OsRcaßD173A/OsRca2 hetero-oligomer, which prevented us from drawing conclusions about its function. It is possible that the low ATPase activity of the hetero-oligomer was not enough to activate Rubisco or the functionality of Rca2 could only be confined to its ATPase function.

Repeating the mixing experiment between Rca2 and another ATPase inactive Walker A mutant (K115A) yielded identical result at an equimolar concentration (Fig. 6.6D). In contrast, OsRca2 did not form a functional hetero-oligomer with the Rca interface mutant (R293V), which appeared unable to exchange subunits in earlier experiments (section 5.5) (Fig. 6.6D). This suggests that the detection of ATPase activity in case of hetero-oligomer was the result of subunit interaction and not an artefact, where OsRca $\beta$ subunit is restoring the functionality of OsRca2. However, formation of complex between Rca 2 and Rca $\beta$ could not be demonstrated using native-PAGE (Fig. $6.6 \mathrm{E})$. It is possible that either the population of hetero-oligomers were too small to be detected or the hetero-oligomeric band could be masked by the overlapping bands of the individual protein. In future, an optimized study with titrated amount of loaded proteins could provide a clearer picture. 

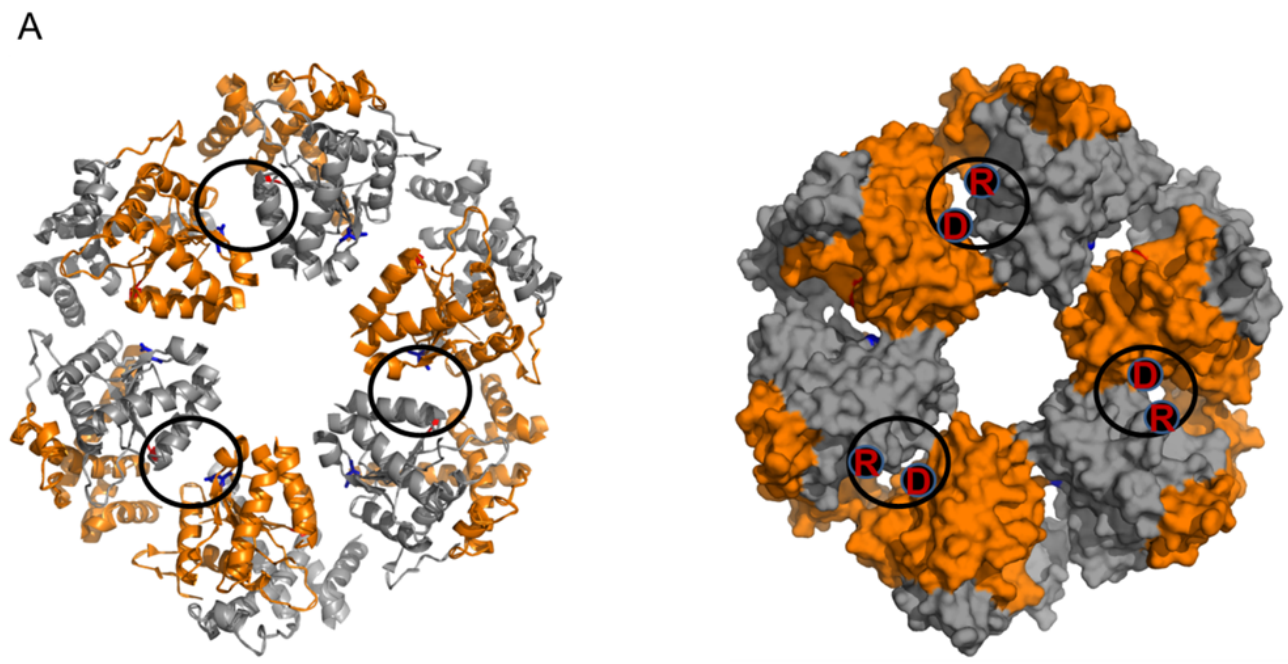

B
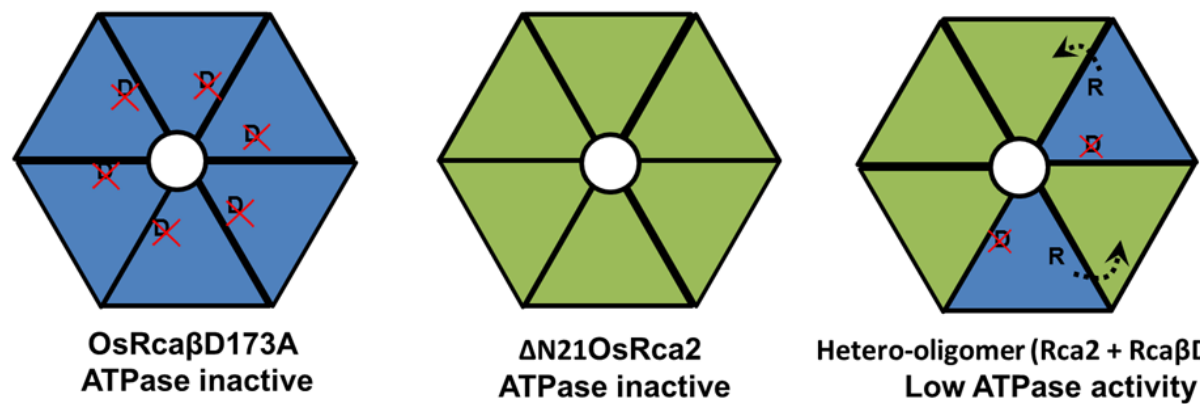

Hetero-oligomer (Rca2 + RcaßD173A) Low ATPase activity

Fig. 6.7: Hypothesized scheme for the formation of functional hetero-oligomer between OsRca2 and OsRcaßD173A. (A) Ribbon and surface representation of the tobacco Rca hexamer highlighting the location of D173 and arginine finger residue R243 (position as per OsRca). (B) OsRcaßD173A and $\Delta \mathrm{N} 21$ OsRca2 were non-functional by their own. The active site is formed by two subunits of the hetero-hexamer when arginine finger comes from OsRcaßD173A. Hexamers are for illustration purpose only.

The restoration of functionality of OsRca2 could be explained on the basis of formation of a hetero-oligomeric active site, where OsRcaßD173A provides its arginine finger (R243 in rice activase) to OsRca2 and makes it functional (Fig. 6.7A and B). The arginine finger in many AAA+ proteins has been implicated in sensing the nucleotide binding state of adjacent subunit and mediating inter-subunit communication $[121,122]$. In tobacco Rca structure, R243 is located adjacent to the Walker B site (D173A) of the neighbouring subunit (Fig. 6.7A) and substituting it with alanine in tobacco has been reported to abolish ATPase activity and nucleotide dependent conformational changes [165]. Therefore, we hypothesize that during 
hetero-oligomer formation; the arginine finger from OsRcaßD173A allows ATP dependent conformational changes in OsRca2, leading to formation of functional active site (Fig. 6.7B). This assumption could be verified in future by performing the subunit mixing with an arginine finger mutant (R243A) of OsRcaß, which should not permit rescue of the ATPase function of the hetero-oligomer. We also attempted to generate Walker $\mathrm{B}$ mutant of $\Delta \mathrm{N} 21$ OsRca2 (D178A) for biochemical study, hypothesising that mixing experiment in this case should eliminate the function of the hetero-oligomer. However, the protein co-eluted with lot of contaminating proteins, which could not be separated even after several chromatographic attempt.

\subsection{Demonstration of a novel complex between $\Delta \mathrm{N} 210$ sRca2 and OsRca $\beta$ when co-expressed in E.coli}

Attempts to demonstrate the functionally observed Rca2/Rca $\beta$ complex by gel filtration were inconclusive. Therefore, we performed a co-expression of $\Delta \mathrm{N} 21$ OsRca2 and its Walker B mutant (D178A) with OsRca $\beta$ to observe the interaction behaviour of Rca2 with Rca 3 . As hypothesized, $\Delta \mathrm{N} 210$ sRca2 was coeluted with activase after anion exchange chromatography, which was further confirmed using analytical gel filtration chromatography (Fig. 6.8A and B). Both Rca2 and Rcaß appeared in all eluted fractions and could not be separated from each other. However, two overlapping peaks were seen, where the peak of Rca2 eluted at similar volume, while the activase peak kept shifting with concentration (Fig. 6.8B). In spite of this, almost all the fractions contain both the proteins. On the other hand, $\Delta \mathrm{N} 21$ OsRca2D178A also co-eluted with activase; however analytical gel filtration chromatography showed that the mutant forms soluble higher ordered oligomers. Still, co-elution of both, mutant and activase was observed in all fractions here also (Fig 6.8B). 
A

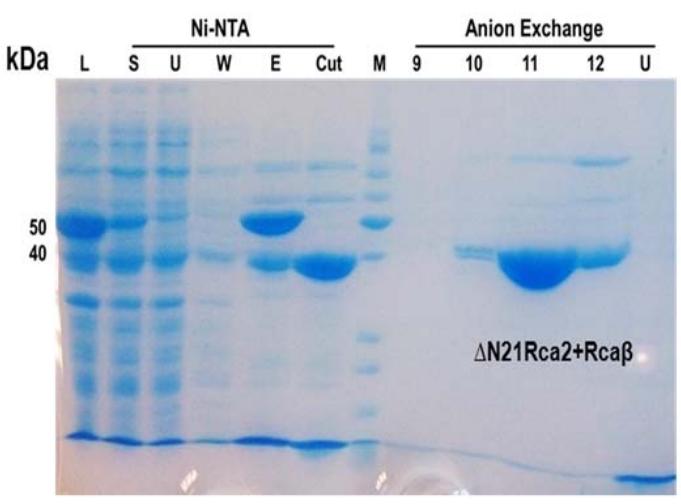

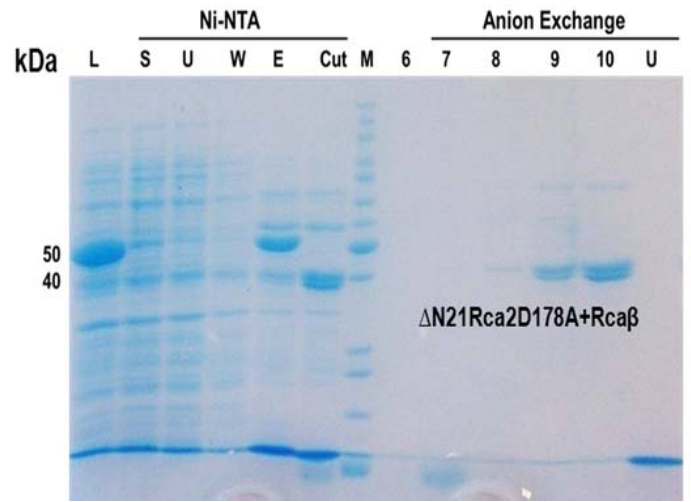

B
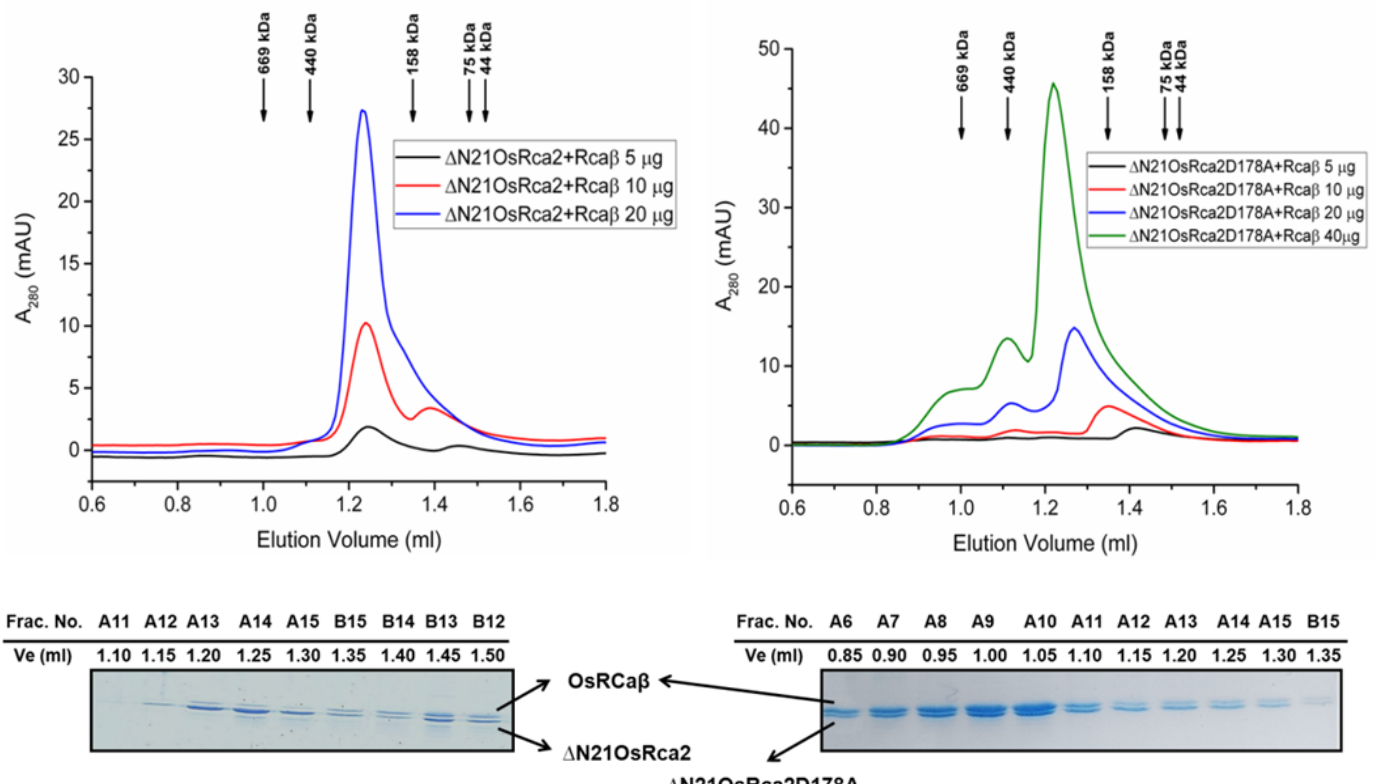

$\triangle \mathrm{N} 210$ sRca2D178A

Fig. 6.8: Co-expression studies between $\triangle \mathrm{N} 210$ sRca2 and Rcaß. (A) Representative purification of $\Delta \mathrm{N} 21$ OsRca2 (left) and its Walker B mutant $\Delta \mathrm{N} 21 \mathrm{OsRca} 2 \mathrm{D} 178 \mathrm{~A}$ (right) co-expressed with OsRca . $12.5 \%$ SDS-PAGE analysis of the co-expressed protein. Successive purification was performed using IMAC (Ni-NTA), followed by Usp2 mediated cleavage of N- terminal tag, dialysis and anion exchange chromatography (AIEC). (M: Molecular Weight Marker, L:Lysate, S:Soluble, Ni-NTA: fractions after IMAC, U: Unbound, W:Wash,: E: Final eluate, Cut : eluate after USP2 mediated cleavage of Ubiquitin. (B) Analytical gel filtration chromatography of $\Delta \mathrm{N} 21 \mathrm{OsRca} 2$ (left) and $\triangle \mathrm{N} 21$ OsRca2D178A (right) co-expressed with OsRcaß performed using a Superdex 200 3.2/30 in 20 $\mathrm{mM}$ Tris-HCl pH 8.0, $50 \mathrm{mM} \mathrm{NaCl}, 5 \mathrm{mM} \mathrm{MgCl} 2,0.5 \mathrm{mM}$ ATP. $12.5 \%$ SDS-PAGE analysis of the samples from the fractions obtained from (B) were shown below their respective chromatogram. Each fraction was $50 \mu \mathrm{l}$ in volume correspondingly.

The co-expressed $\Delta \mathrm{N} 21$ OsRca 2 and $\Delta \mathrm{N} 21$ OsRca2D178A with OsRca $\beta$ was also analyzed using native PAGE and western blot (Fig. 6.9A and B). Rca2 was detected using the anti-activase antibody; however the western blot signals for Rca2 were 
much weaker than the activase signal (Fig. 6.9A). In case of co-expressed protein, bands for Rca2 and Rca $\beta$ were clearly seen using the native PAGE and western blot, where the relative position of Rca2 bands does not changes, while a visible shift was observed for Rca $\beta$ with the concentration (Fig. 6.9B). In case of $\Delta \mathrm{N} 21$ OsRca2D178A, the mutant protein appeared to run higher on the gel and the Rca $\beta$ bands were seen as usual, suggesting that $\Delta \mathrm{N} 21$ OsRca2D178A was forming higher order oligomers even in presence of Rca $\beta$.

A

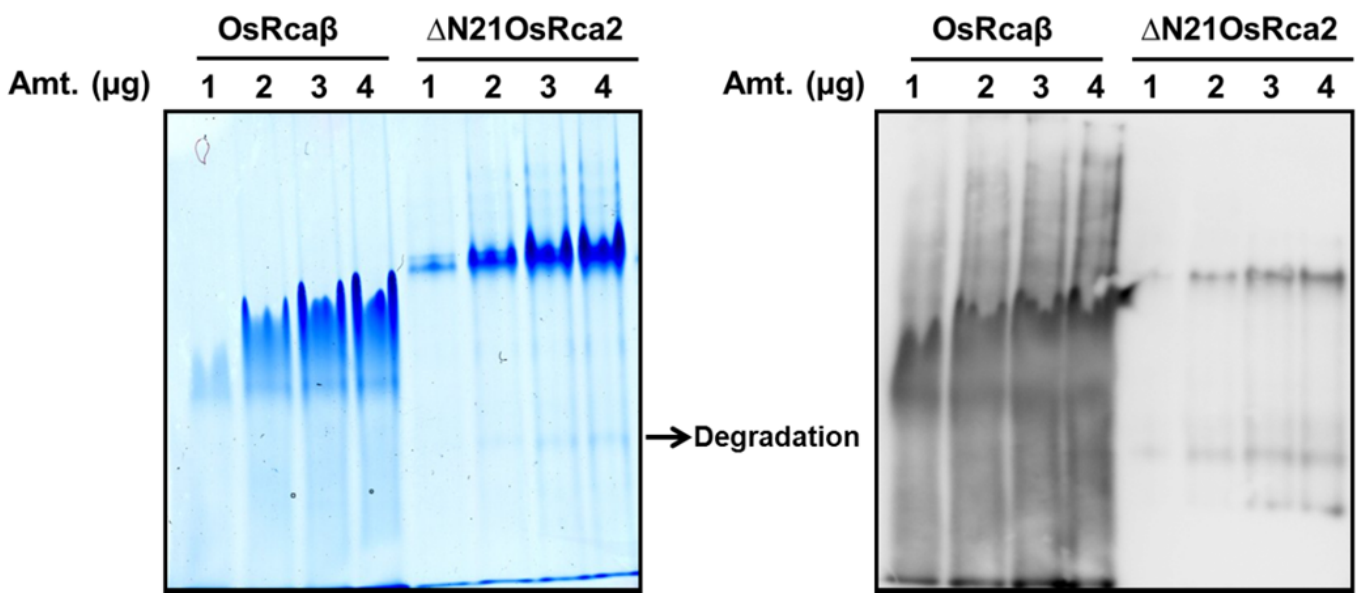

B

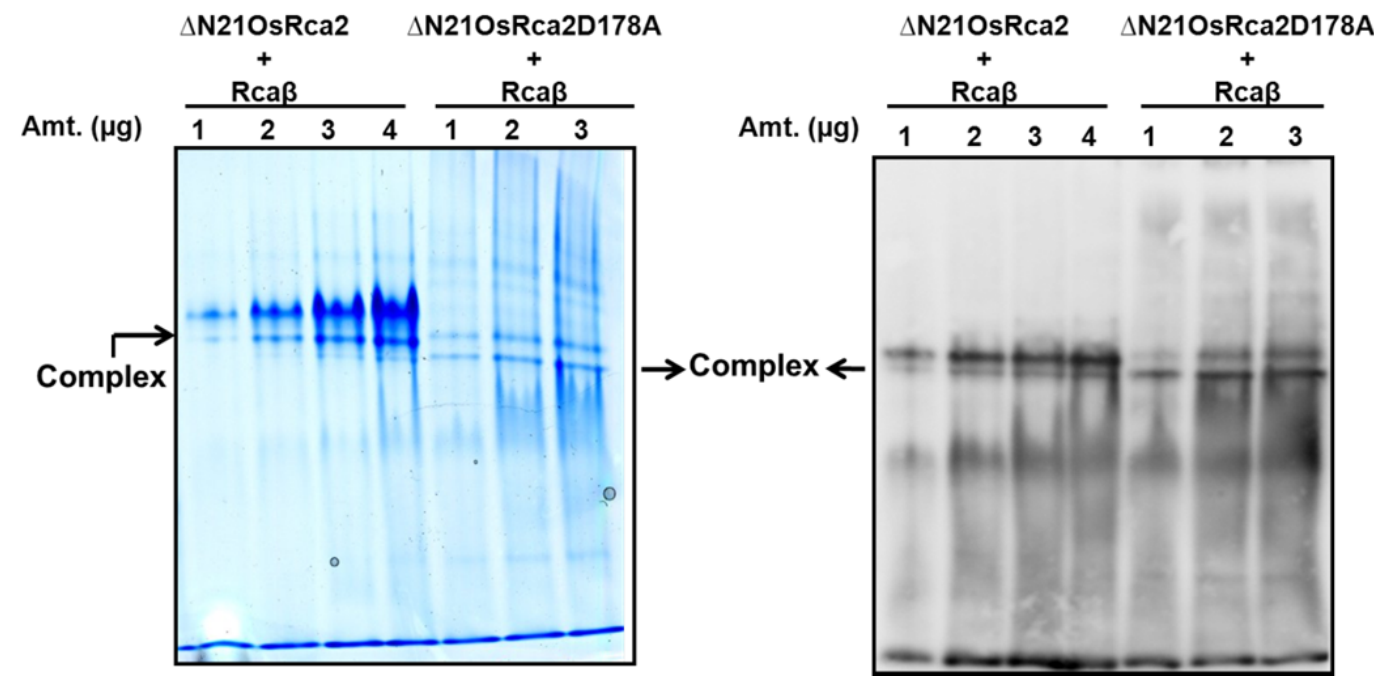

Fig. 6.9: Complex formation between $\Delta \mathrm{N} 210$ sRca 2 and Rca $\beta$ during co-expression. (A) Native PAGE (left) and western blot (right) analysis of OsRca $\beta$ and $\triangle$ N21OsRca2. (B) Native PAGE (left) and western blot (right) analysis of $\Delta \mathrm{N} 21 \mathrm{OsRca} 2$ and $\triangle \mathrm{N} 21 \mathrm{OsRca} 2 \mathrm{D} 178 \mathrm{~A}$ co-expressed with OsRca $\beta$ demonstrating formation of complex between OsRca $\beta$ and $\Delta \mathrm{N} 21$ OsRca2 through additional band. Loaded amount is indicated above each lane. 
Interestingly, in case of these co-expressed proteins, there was an additional band between the original Rca2 and Rca $\beta$ bands, which could correspond to a complex between Rca2 and Rca $\beta$. This band was not observed when Rca2 and activase were run in isolation (Fig. 6.9A). However, some experiments are needed to validate this outcome. Next steps will involve attempts to isolate the complex in a homogenous preparation.

In summary, this section demonstrated a preliminary attempt to characterize the putative Rubisco activase isoform, Rca2. So far, most studies concerning this isoforms were confined to detection in high-throughput approaches. To our knowledge, it was the first attempt where this protein was obtained in purified form and considered for characterization. The results presented here indicate some relationship between Rca2 and Rcaß. Although, it would be too early to give a conclusive statement on the function of Rca2, still this work generated a hint towards its functional characterization.

Although we have left many unanswered questions regarding Rca2 due to time constraints, the present study could be taken advantage for the future work in the laboratory to fill the gaps. For instance, further biochemical characterization of the Rca2 with other activase mutants such as interaction site mutants could be utilised to find if Rca2 is a component or modulator of plant Rubisco activase or not. Apart from that the monodispersity of Rca2 projects it as an ideal candidate for structural studies using cryo-electron microscopy. In future, attempts should be made to obtain a high resolution structure of Rca2 alone and also in complex with Rca2-Rca or Rca2Rubisco for its structural characterization. Also, in vivo approaches such as purifying activase from the plant source and analyze whether Rca2 co-purifies with activase using mass spectrometric techniques might provide evidence for in vivo interaction among Rca2 and activase. The Rca2 knockout seeds are available commercially and could be used for phenotypic study in plants. Collectively, positive outcomes glean through such approaches would endeavour to characterise Rca2 biochemically and unfold the mystery behind its in vivo function. 


\section{Discussion}


Rubisco activase (Rca) is a key photosynthetic enzyme as it maintains the CalvinBenson cycle flux in plants by remodelling of inhibited Rubisco, using energy from ATP hydrolysis. The thermolability of Rubisco activase appears to limit photosynthesis capacity of plants under moderate heat stress $[10,11]$. The increasing global temperature due to climate change will further exacerbate this problem [18]. A comprehensive understanding underlying the mechanistic basis of activase thermostability and function is much needed to counter the predicted adverse effects of global warming.

In this study, we have made an effort to improve our appreciation of plant Rubisco activase function from a biochemical perspective, using rice as a model system. The work presented here has focussed on a detailed characterization of rice Rubisco activase followed by a comparative study with thermostable Rca homologues from a CAM plant, Agave tequilana. Moreover, extensive mutagenesis based on previous studies and with aid from available structure were utilised to enhance our mechanistic appreciation of the Rubisco activation system in this economically important crop.

Here, we place our results in context with the newly published results on rice activase [177] and previous studies on other plant systems. Finally, we present an updated model for the mechanism of Rubisco activation .

\subsection{Rice activase isoforms exhibit typical regulatory behaviour and have similar thermostability to other characterized homologues}

The data generated here represent the detailed biochemical characterization of two Rubisco activase isoforms from the key crop species rice. The biochemical information regarding rice activase was completely unavailable when this study was initiated. However, recently, a biochemical and thermal characterization of two rice species (Oryza sativa and Oryza australiensis) was published [177]. This study mainly focussed on concentration and temperature dependent activity of Rca. Consistent with this study, we found that both activase isoforms are capable of hydrolysing ATP and remodelling Rubisco with differential ability in the oxidized state. OsRca $\beta$ was significantly more active than OsRca $\alpha$ in terms of both activities at tested concentration (Fig. 3.4). This trend was also reported in the recent study at 
similar concentration ranges [177] and also for arabidopsis in earlier studies [146], where the $\beta$ isoform possessed higher activities than the $\alpha$ isoform. However, for both rice (Fig. 3.5) and arabidopsis, the $\alpha$ isoform shows enhanced activity under the reducing effect of DTT and thioredoxin-f, while no effect was observed on the $\beta$ isoform [146]. Thus, the $\alpha$ isoform has the ability to modulate its activity under redox state via thioredoxin-f, providing a mechanism for the light regulation of Rubisco as first discovered in arabidopsis [146]. This property of the $\alpha$ isoform is due to the presence of two conserved cysteine residues at its $\mathrm{C}$-terminal extension, which are capable of forming a disulphide bond.

It has been reported that the thermal stability of Rca varies between different species, and there is a direct correlation between the heat tolerance of photosynthesis and the thermal stability [169]. Our data on the thermal characterization of Rubisco activases revealed that rice exhibits a very similar thermostability within its activase isoforms with the $\beta$ isoform being slightly more thermostable than the $\alpha$ isoform (Fig. 3.7). The $\alpha$ and $\beta$ isoforms lost half of their activity at $37^{\circ} \mathrm{C}$ and $40{ }^{\circ} \mathrm{C}$ respectively. In Scafaro et al's work, the loss of ATPase hydrolysis was also reported after temperature exceeded $36-40{ }^{\circ} \mathrm{C}[177]$.

In other species like cotton, the $\alpha$ isoform is found to be slightly more thermostable than its $\beta$ isoform but thermostability within its activase isoform does not differ greatly unlike spinach, where the $\alpha$ isoform is much more thermostable than its $\beta$ isoform $[141,147,178]$. These differences in thermostability in rice and other species seem interesting and suggested some physiological significance. The $\alpha$ isoform in rice was reported to be upregulated during heat stress at mRNA level and found to be most upregulated protein in the wild rice species Oryza meridionalis [196, 197]. However, similar effects were not observed in arabidopsis [212]. It was hence tempting to speculate that altered $\alpha$ and $\beta$ isoform abundance in rice with heat could be a mechanism to downregulate Calvin-Benson cycle in response to temperature and redox changes, where low activity OsRca $\alpha$ modulates the activity of OsRcaß. Heat stability of the $\alpha$-isoform did not alter with its redox state as shown in this study (section 3.6). Unlike in spinach, where Rca $\alpha$ confers thermostability to the $\beta$ isoform $[141,178]$, no such effect was observed in our study (section 3.6). Thus, the hypothesized role for the $\alpha$ isoform to transfer high thermostability to the $\beta$ isoform 
was not confirmed in rice and the primary role of the $\alpha$ isoform appears to be confined to redox regulation of activase. Similarly, in arabidopsis, the $\alpha$ isoform was not observed to be more thermostable than $\beta$ the isoform [166].

The structural integrity of Rca isoforms was also assessed by circular dichroism spectroscopy (CD). The $\mathrm{CD}$ analysis confirmed that the mid-point for thermal unfolding lies at about $35-40{ }^{\circ} \mathrm{C}$ for both Rubisco activase isoforms of rice (Fig. 3.8). The earlier CD studies also denoted the similar thermostability for the $\beta$ isoform of arabidopsis with mid-point for thermal unfolding falls around $40{ }^{\circ} \mathrm{C}[134,138]$. The thermal mid-point for protein denaturation was approximated at $45{ }^{\circ} \mathrm{C}$ for the $\beta$ isoform of cotton and tobacco, while $46-47^{\circ} \mathrm{C}$ for creosote Rca $\beta$ in the reported study [138]. All these CD studies were performed in presence of $\mathrm{Mg}^{2+}$ and ADP, while we have performed all our experiments in apo form.

It has been reported that nucleotide binding plays a role in Rca stability as ADP and ATP provide protection against heat inactivation to the $\beta$ isoform of Rca in arabidopsis [134] and cotton [138]. ADP was found to increase the thermal stability of spinach Rca isoforms, while ATP $\gamma \mathrm{S}$, a non-hydrolysable analogue of ATP, caused a substantial increase in the thermal stability of the $\alpha$ isoform of spinach $[141,178]$. Therefore, in future, a similar characterization of the effect of nucleotides and metal ions should be performed for rice. Nevertheless these results along with observations from the native PAGE analysis (Fig. 3.9A) in our study showed a clear relation between the structural instability of Rca at high temperature and its activity in rice.

\subsection{Subunit exchange and cooperativity between Rca isoforms}

Some AAA+ ATPases including Rca can exchange subunits in vitro and therefore subunit mixing experiments were carried out between the isoforms to observe the mutual regulatory effect of the isoforms and to probe the mechanism employed in their function. Consistent with the arabidopsis model [146], it was observed that the ATPase activity of OsRca $\beta$ was continuously reduced with increasing concentration of OsRcad (Fig. 3.10), clearly indicating the interaction between the two isoforms. However, to deconvolute the results, similar experiments were performed in the presence of ATPase inactive mutants of the both isoforms. Use of these mutants revealed that subunits interact irrespective of their types and follow a concerted or 
sub-globally cooperative mechanism of ATP hydrolysis (3.11C). The interaction between OsRca $\beta$ and OsRca $\alpha$ was further validated in the study using analytical gel filtration chromatography (Fig. 3.12). Previously, the evidence for interaction between Rca $\alpha$ and Rca $\beta$ has been presented for arabidopsis and spinach using subunit mixing $[141,146,155,178]$. Recently, interaction between the $\alpha$ and the $\beta$ isoforms subunits in sweet potato has also been suggested using yeast two hybrid system [260].

In vitro mixing experiment between $\mathrm{Rca} \beta$ and $\mathrm{Rca} \alpha$ in rice supports a regulatory influence of $\alpha$ isoform on the $\beta$ isoform. This provides a mechanism to modulate the ATPase activity of the $\beta$ isoform by the $\alpha$ isoform in vivo by rapidly forming the hetero-oligomeric mixture of low activity Rca $\alpha$ and high activity Rca $\beta$. Since in rice, Rca $\beta$ is much more abundant than Rca $\alpha$ under normal conditions [144], modulating the concentration Rca $\alpha$ allows activity of the hetero-oligomer to be regulated under different redox state and other stressful conditions. In Arabidopsis, two Rca isoforms occurs in 1:1 ratio, while tobacco possesses only the $\beta$ isoform. This differential stoichiometry of two isoform in plants could have different physiological significance. In arabidopsis, it has been demonstrated that the $\beta$ isoform gets completely inhibited in the presence of $\alpha$ isoform at the equimolar concentration at an ATP/ADP ratio of $1: 3$ [155] indicating that the $\alpha$ isoform allosterically regulates activity of the $\beta$ isoform. While in tobacco, which only expresses the $\beta$ isoform, ADP shows strong competition with ATP resulting in reduction of ATPase activity [154]. The unusual stoichiometry of rice Rca under normal conditions, and its altered abundance under heat stress reflects that rice might represent a phenotype which could be intermediate to arabidopsis and tobacco. It is possible that either rice Rca may not completely deactivate in vivo or both isoform might possess intermediate level of sensitivity towards ATP/ADP ratio.

In summary, contrary to our hypothesis rice isoforms do not exhibit significant difference in thermostability and the upregulation of the $\alpha$ isoform is most likely a mechanism to reduce the activity of the $\beta$ isoform under abiotic stress conditions. In future, the effect of nucleotides on subunit mixing and behaviour of different Rca under variable ATP/ADP ratio needs to be investigated further to perceive and compare the effects. Also, a high resolution structure of the $\alpha$ isoform alone and the 
Rca $\alpha$-Rca $\beta$ complex with intact C-tail extension in reduced and oxidized state would be extremely helpful in resolving these issues.

\subsection{Comparison between biochemical and thermal properties of rice (C3) and agave (CAM)}

This study includes the first characterization of the Rubisco activase system from a CAM plant. The physiological properties of CAM plants suggested the possibility of encountering natural thermostable activases as per our hypothesis. Agave tequilana possesses two Rca isoforms like many other plants. However unlike rice and arabidopsis, where two isoforms are the result of alternate splicing $[112,143]$ the two isoforms in agave are produced from different genes as described in cotton [147].

Studies on activase from spinach and arabidopsis reported that the $\beta$ isoform possesses a higher Rubisco activation rate than their respective $\alpha$ isoform $[146,148]$. Arabidopsis follows similar trend also in term of its ATPase activity, while both isoforms of spinach have almost similar ATPase rate [178]. This comparative study between rice and agave showed that the $\beta$ isoform of rice was significantly more active than the $\alpha$ isoform in the oxidized state, a phenotype more similar to arabidopsis. Interestingly, in agave both isoforms were almost equally active in terms of their ATPase as well as Rubisco activation activity (rice Rubisco). Agave exhibited higher Rubisco (rice) activation rates in vitro compared to the cognate OsRca (section $4.2)$.

The experiments testing the effects of molecular crowding performed in the study indicated a higher affinity between agave Rca and rice Rubisco as compared to rice Rca itself. Thus, introducing a high affinity activase from agave into rice could be a possible approach in future, as less activase may be required to activate the same amount of Rubisco. However, in the crowded stromal environment high affinity could result in reduced activase activity due to a slow off-rate and these issues therefore need to be carefully evaluated in future.

Subsequent thermal characterization of activases revealed that the $\beta$ isoform of agave maintained significant ATPase hydrolysis activity at $50{ }^{\circ} \mathrm{C}$, whereas most of the OsRca functionality was lost at $42{ }^{\circ} \mathrm{C}$ (section 4.3). Considering the problem 
associated with the thermolability of plant activases such outcomes are very promising. The thermostability observed for agave activases in our study was to date the highest for any reported plant activase system isoform in terms of their stability in the apo form (Table 7.1). Recently, a hetero-oligomeric red-type activase from the thermophilic red algae, Cyanidioschyzon merolae has been reported to be fully functional up to $55^{\circ} \mathrm{C}$, however it was unable to activate plant Rca [104].

The previous data in the literature on activase thermostability is quite complex and measured in various ways such as in terms of ATPase activity, CD, light scattering thermofluor shift assay etc. Furthermore, the experimental conditions employed in the studies were also variable and complicate comparative analysis. A summary of the information available on activase thermostability in various plant systems has been tabulated in Table 7.1.

Earlier studies reported that the $\alpha$ isoform of spinach, which is a temperate plant, possessed enhanced thermostability with a $\mathrm{T}_{\mathrm{m}}$ of $55^{\circ} \mathrm{C}$ in presence of $\mathrm{ATP} \gamma \mathrm{S}$, while the increase was not so significant for the $\beta$ isoform $\left(41{ }^{\circ} \mathrm{C}\right)$ [178]. Similar thermostability profiles were replicated for spinach using the thermofluor shift assay [141]. Thus, spinach Rca $\alpha$ demonstrates a unique property in thermostability among its Rca isoform in the presence of $\mathrm{Mg}-\mathrm{ATP} \gamma \mathrm{S}$, as such a substantial difference in the thermostability of the two isoforms has not been reported for any other plant Rca system. However, both the isoforms in spinach were extremely sensitive to a moderate increase in temperature in their apo form with a $\mathrm{T}_{50}$ of $30^{\circ} \mathrm{C}$, much lower than agave and other plants species isoforms (Table 7.1). It is interesting to note that the second most thermostable activase so far was found in creosote bush, which is a desert plant, followed by tobacco, the tropical plant, while temperate plants, like arabidopsis possess lower thermostability. Taken together our study on agave, which is a CAM plant, further supported the reasonable assumption that the thermostability of Rca varies from warmer to cooler climatic region $[15,169]$. 


\begin{tabular}{|c|c|c|c|c|c|c|}
\hline Organism & State & $\begin{array}{c}\text { Full } \\
\text { ATPase } \\
\text { activity } \\
\left({ }^{\circ} \mathrm{C}\right) \\
\end{array}$ & $\begin{array}{c}\mathrm{T}_{50} \\
\left({ }^{\circ} \mathrm{C}\right)\end{array}$ & $\begin{array}{c}\text { Onset of } \\
\text { protein } \\
\text { denaturation } \\
\left({ }^{\circ} \mathrm{C}\right) \\
\end{array}$ & $\begin{array}{c}\text { Apparent } \mathrm{T}_{\mathrm{m}} \\
\left({ }^{\circ} \mathrm{C}\right) \\
(\mathrm{CD} / \mathrm{TF})\end{array}$ & Citation \\
\hline Agave $(\alpha)$ & Apo & 40 & 45 & ND & ND & This study \\
\hline Agave $(\beta)$ & Apo & 45 & 50 & ND & ND & This study \\
\hline $\begin{array}{l}\text { Arabidopsis } \\
(\beta)\end{array}$ & Apo & 25 & 30 & $\begin{array}{c}\sim 32 \\
\text { (Mg-ADP) }\end{array}$ & $\begin{array}{c}\sim 40 \\
\text { (Mg-ADP) }\end{array}$ & {$[12,134,138]$} \\
\hline Cotton $(\beta)$ & Apo & 33 & 40 & $\begin{array}{c}\sim 40 \\
\text { (Mg-ADP) }\end{array}$ & $\begin{array}{c}\sim 45 \\
\text { (Mg-ADP) }\end{array}$ & {$[138,169]$} \\
\hline $\begin{array}{l}\text { Creosote } \\
\text { bush }(\beta)\end{array}$ & $\mathrm{ATP} \gamma \mathrm{S}$ & 41 & 43 & $\begin{array}{c}\sim 40 \\
\text { (Mg-ADP) }\end{array}$ & $\begin{array}{r}\sim 46-47 \\
(\mathrm{Mg}-\mathrm{ADP})\end{array}$ & {$[138,169]$} \\
\hline $\begin{array}{l}\text { Antarctic } \\
\text { hairgrass }\end{array}$ & $\mathrm{ATP} \gamma \mathrm{S}$ & 35 & 38.5 & ND & ND & [169] \\
\hline $\operatorname{Rice}(\alpha)$ & Apo & 35 & 37 & $\sim 35$ & $\sim 40$ & This study \\
\hline Rice $(\beta)$ & Apo & 35 & 40 & $\sim 35$ & $\sim 40$ & This study \\
\hline $\operatorname{Spinach}(\alpha)$ & $\begin{array}{c}\text { Apo/Mg- } \\
\text { ATP } \gamma \mathrm{S}\end{array}$ & $25 / 40$ & $30 / 55$ & ND & $\begin{array}{c}\text { 25/30/55 } \\
\text { (apo/Mg- } \\
\text { ADP/Mg- } \\
\text { ATP } \gamma \text { S) }\end{array}$ & {$[141,178]$} \\
\hline Spinach $(\beta)$ & $\begin{array}{c}\text { Apo/Mg- } \\
\text { ATP } \gamma \mathrm{S}\end{array}$ & $25 / 40$ & $30 / 41$ & ND & $\begin{array}{c}25 / 40 / 40 \\
\text { (apo/Mg- } \\
\text { ADP/Mg- } \\
\text { ATP } \gamma \text { S })\end{array}$ & {$[141,178]$} \\
\hline Tobacco & Apo & 35 & $40-42$ & $\begin{array}{c}\sim 40 \\
(\mathrm{Mg}-\mathrm{ADP})\end{array}$ & $\begin{array}{c}\sim 45 \\
(\mathrm{Mg}-\mathrm{ADP})\end{array}$ & {$[138,169]$} \\
\hline
\end{tabular}

Table 7.1: Comparison of the in vitro data on thermostability of Rca among higher plants. *ND = not determined, $\mathrm{CD}=\mathrm{Circular}$ dichroism, $\mathbf{T F}=$ thermofluor assay 
It was hypothesized that conformational flexibility of Rca is required for it to remodel Rubisco conformation and this is likely be the reason why Rca cannot be thermostable [15]. Research comparing a warm-season plant (cotton) and a cool-season plant (Camelina sativa) also showed that there was a trade-off between Rca thermostability and its activity at low temperature $[15,229]$. The recent data published on rice further reported similar compromise between thermostability and activity among its two species [177]. However, this is not true for agave activase and no trade-off was observed in terms of their Rubisco activation activities as well as ATPase activity at low temperature compared to rice (section 4.3).

Our results thus contradict a long standing argument pertaining to the thermostability of Rca and engendered a hope that it is possible to find both thermostable and functionally efficient Rca at the same time in the natural environment. The high functionality of agave activases at both low and high temperature could be related to the North American desert environment, where temperature in the light can fluctuate between broader ranges in summer $\left(20-45^{\circ} \mathrm{C}\right)$ and in winter $\left(2-25^{\circ} \mathrm{C}\right)$ [261]. It is possible that in similar species exposed to such a wide alteration in temperature regime as seasonal and diurnal variation, a strong selection pressure has been acting to maintain activase functionality at both high and low temperatures.

Our work was motivated to explore the future possibility of heterologously expressing more thermostable activases in rice. In earlier work, overexpression of Rca in rice resulted in improved activation state of Rubisco and rate of carbon dioxide assimilation with respect to wild type at fluctuating light conditions [230, 262]. Overexpression of barley Rca in rice plants led to reduced activation state under ideal conditions [262]. On the contrary, overexpression of maize Rca resulted in better activation state and $\mathrm{CO}_{2}$ assimilation at variable light intensity [230]. This suggests, a more effective and compatible Rca could provide benefit to the host plants.

In our study, we have observed that although rice and agave Rca readily formed hetero-oligomeric complexes, activase functionality was not perturbed in terms of both ATPase rate as well as Rubisco action activity. Upon heating the heterooligomer, agave subunits remained in solution and functional while rice Rca formed inactive higher order aggregates (section 4.4). Therefore, we hypothesize that agave 
Rca overexpressed in rice plants in vivo might replicate similar effects and could result in plants with enhanced photosynthetic performance at elevated temperatures.

There has been a controversy regarding the factors limiting photosynthesis at high temperature. However, thermolability of Rca and limitations of electron transport chain are considered as most debatable [10, 16-18, 169, 171, 263]. Expressing thermostable variants of activases have demonstrated improved growth and photosynthetic potential at supraoptimal temperature $[12,13]$. However, it could be argued that in normal $\mathrm{C}_{3}$ plants like rice, having a more thermostable Rca could actually decrease the efficiency of photosynthesis. This is because as temperature rises, the rate of photorespiration increases faster than the rate of photosynthesis [16]. Unlike $\mathrm{C}_{4}$ and $\mathrm{CAM}$ plants, $\mathrm{C}_{3}$ plants do not have a carbon-concentrating mechanism (CCM). Thus, when they close their stomata at high temperature to prevent water loss; there will be insufficient $\mathrm{CO}_{2}$ entering the leaves. Moreover, the solubility of $\mathrm{CO}_{2}$ and Rubisco specificity $\left(\mathrm{CO}_{2} / \mathrm{O}_{2}\right)$ decreases with high temperature favouring production of misfired products [99, 103]. In such a situation, having a functional thermostable activase will result in increased photorespiration which is a wasteful process. Therefore, having a thermolabile Rca may serve as a regulatory mechanism to stop Rubisco from functioning at high temperature $[16,17]$, especially when the rate of photorespiration exceeds the rate of photosynthesis.

However, under agricultural care, plants can overcome the problem of photorespiration if enough water is provided as they would be able to keep their stomata open at high temperature without being dehydrated. In this situation, the thermostability of Rca could become a limiting factor. Since the problem of photorespiration is ameliorated, having a more thermostable Rca should allow Rubisco to stay active at higher temperature, increasing the efficiency of photosynthesis.

In earlier studies, accumulation of glycinebetaine in chloroplasts and overexpression of the enzyme SBPase have been associated with increased Rca stability in tobacco and rice respectively [264, 265]. Another genetic engineering approach utilised silencing of a specific $\omega-3$ fatty acid desaturase gene, which caused reduction of thylakoid membrane lipid unsaturation. This strategy has also proved to be effective for the host plant by improving its growth and photosynthetic at elevated temperature 
$[263,266]$. Taken together, our study along with previously existing ones suggested possible solution pertaining to the thermosensitivity of photosynthesis. Expressing agave Rca in rice could provide a good experimental system to further evaluate the in vivo impact of such strategies and interrogate whether photosynthesis is more limited by the thermolability of Rca or other factors. In fact a collaboration has been initiated to probe these issues

\subsection{Insight into thermostability and Rubisco activase mechanism gleaned through bioprospecting}

In an attempt to locate specific regions/residues that confer thermostability, chimeric mutants were generated by interchanging the domains of Rubisco activase between rice and agave (section 4.5). Domain swapping experiments localized thermostability to the nucleotide binding domain (NBD) of AtRca. The chimeric mutant possessing the NBD of agave Rca not only exhibits $5-7^{\circ} \mathrm{C}$ higher thermostability but also $\sim 4$ fold higher Rubisco activation in vitro for rice activase compared to OsRca.

An earlier study generated a chimeric activase by replacing the specificity domain of tobacco with arabidopsis [13]. The chimeric mutant (Tob-Arab) in that study acquired higher thermostability of tobacco, while the rate of Rubisco activation was similar to wild type arabidopsis Rca under standard in vitro assay conditions. Thus our strategies have observed to confer dual benefit in terms of thermostability and Rubisco activation rate (in vitro). Also the results from the previous study further supported our assumption that the NBD contributes significantly to the thermostable properties of activase. It was interesting to note that one of the residues from the sensor-2 region (C-terminal domain) was observed to affect thermostability in the gene shuffling strategy employed previously [12]. However, these variants were generated as second round mutant, also consisting an amino acid substitution from NBD out of total three residues. The in vitro phenotype of variant consisting single residue substitution (T274R in arabidopsis) did not yield a significant result at high temperature compared to WT in the same study. Similarly, no tested single residue substitutions could be identified to be responsible for thermostability in the NBD region in our study (section 4.7). This indicates that thermostability might be the outcome of tighter packing of structural elements and employed larger segments of 
protein such as domain or motif rather than a single amino acid difference. This postulate is supported by our study taking together previous studies mentioned in this section.

Interestingly, in such attempts we have found that a single glutamic acid (E217) to glutamine (E-Q) substitution localized at a newly identified surface loop of the activase conferred AtRca's high activation rate to OsRca (section 4.7). Examination of the structure of Rca from tobacco [129] revealed that this loop is not resolved but present on the surface at close proximity of helix 9 (specificity region) of the adjacent subunit. Further mutational analysis implicated this loop in Rubisco-Rca proteinprotein interactions and revealed a key lysine residue (K216) adjacent to glutamate (E217), which might be involve in Rubisco-Rca interactions (section 4.8) along with residues from helix 9 as the primary site of interaction. Further bioprospecting and careful biochemical characterization of Rca homologues will lead to both mechanistic insights and identification of candidate proteins for engineering enhanced thermotolerance in rice and other important crops.

\subsection{New insights into the Rubisco activation mechanism}

Our mechanistic understanding of the green-type Rca function is still poorly defined. Various models pertaining to Rubisco-Rca interaction have been proposed in the past based on structural and mutational studies. When the structure of the activase was unknown, a model was proposed where large oligomers of the activase surround the equator of the Rubisco holoenzyme [8]. This early model was superseded by a more detailed structural and biochemical study which proposed the transient hexamer as the functional unit for activase functioning [129]. Based on the structural considerations and mutational data obtained, a model for Rubisco activation was proposed, where the N-terminal domain and helix 9 (specificity helix) are involved in initial recognition of Rubisco as primary site of interactions [59, 129]. Furthermore, partial threading of larger Rubisco segments through central pore was proposed. This model was further supported by another study performed on interspecies-hybrid Rubisco containing large subunit from Chlamydomonas and small subunits from tobacco, spinach and arabidopsis [246]. Based on the biochemical characterization of the chimeras, two hypothetical models were proposed, where a side on model of the Rubisco-Rca interaction was preferred over the top-on geometry as proposed earlier [59, 129]. 
In this work, we have performed a reasonably extensive mutational study to validate previously reported mutational phenotypes in rice, and identified some novel residues that are likely involved in the Rubisco-Rca interaction. The sequence alignment between activases from different plants has been displayed in Fig. 7.1 highlighting the residues mutated in this study. Table 7.2 compiled the list of mutations performed in the present study along with the observed outcome on the functionalities.

We have observed that mutation of residues in the N-terminal domain (W15A, S23A and S23D) and helix 9 (K315D) eliminate activase function supporting the previously proposed mechanism where these domains mediate initial interaction with Rubisco $[59,129]$. Mutations in pore loop $1(\mathrm{~A} 143 \mathrm{~V})$ completely abolished activase function further strengthening the role of pore loop 1 in Rubisco remodelling. We have identified new residues (K148, D159) located further downstream to pore 1 at the surface helix 3, involved in the Rubisco-Rca interaction (section 5.3) along with the surface arginine (321) located at the periphery of the hexamer. Characterization of the newly identified surface loop implicates a key lysine (216) residue in activase/Rubisco interaction (section 4.8).

We have also attempted to rank the relative importance of the key residues by cooperativity analysis using the mutant doping strategy (chapter 5). Our results are consistent with the notion that mutations in the residues or domain thought to be involved in activase-Rubisco interaction can be tolerated more easily (W15, $\Delta$ N67) compared to the mutant subunits containing lesions at mechanistically important sites such as pore loop 1 motif (A143). In the near future, similar mixing experiment will be performed with all newly identified residues to generate a more detailed picture. 

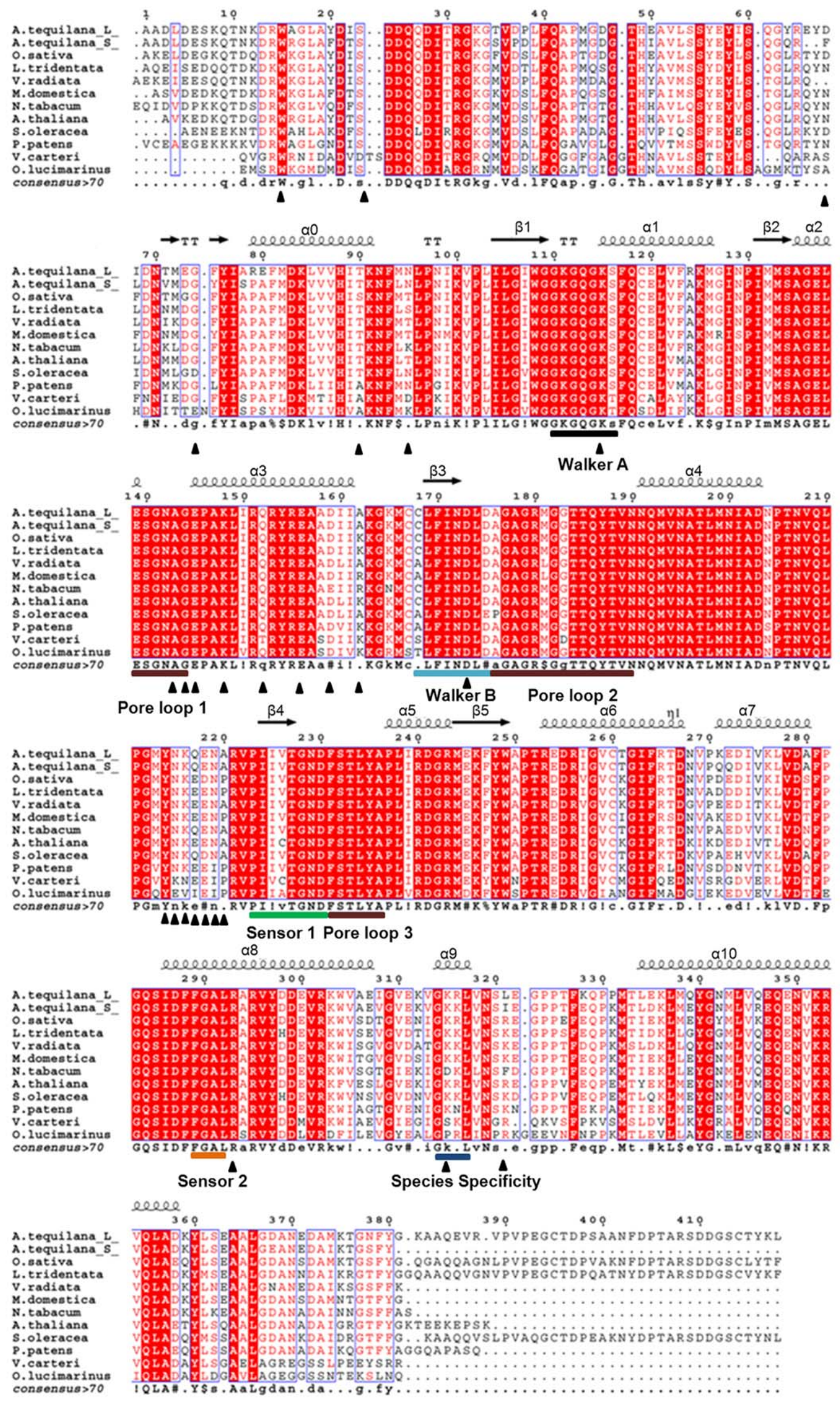
Fig. 7.1: Protein sequence alignment of Rubisco activase from selected species. Amino acid sequences of selected Rubisco activases (Rca) from plants and green algae were aligned using ESPript [184]. The functional motifs and residues analyzed using mutagenesis are indicated using coloured bar and triangles respectively. The secondary structure elements of the tobacco activase (PDB id: 3 T15) has been displayed above the sequence.

\begin{tabular}{|c|c|c|c|}
\hline \multirow{2}{*}{ Mutant (OsRcaß) } & \multicolumn{2}{|c|}{ Inference } & \multirow{2}{*}{ Location } \\
\hline & ATPase activity & Activase activity & \\
\hline W15A & $V$ & $\downarrow$ & $\mathrm{N}$-terminal domain \\
\hline S23A/S23D & $V v$ & $\downarrow \downarrow$ & $\mathrm{N}$-terminal domain \\
\hline F68L & $\downarrow$ & $\downarrow$ & $\mathrm{N}$-terminal domain \\
\hline$\Delta \mathrm{N} 67$ & $\uparrow$ & $\downarrow$ & $\mathrm{N}$-terminal domain \\
\hline G73D & $V$ & $V$ & $\mathrm{~N}$-terminal domain \\
\hline S90T & 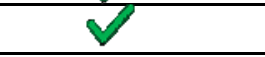 & $V$ & Helix $0(\alpha 0)$ \\
\hline T95N & $V$ & 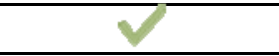 & Between $\alpha 0-\beta 1$ \\
\hline K115A & $\downarrow$ & $\downarrow$ & Walker A \\
\hline $\mathrm{A} 143 \mathrm{~V}$ & $V$ & $\downarrow$ & Pore loop 1 \\
\hline G144A & $\downarrow$ & $\downarrow$ & Pore loop 1 \\
\hline E145A & $\downarrow$ & $\downarrow$ & Helix $3(\alpha 3)$ \\
\hline K148A & $\uparrow$ & $\downarrow$ & Helix $3(\alpha 3)$ \\
\hline Q152A & $\downarrow$ & $\downarrow$ & Helix $3(\alpha 3)$ \\
\hline E156A & $\downarrow$ & $\downarrow$ & Helix $3(\alpha 3)$ \\
\hline D159A & $\downarrow$ & $T$ & Helix $3(\alpha 3)$ \\
\hline K162A & $V$ & $\downarrow$ & Between $\alpha 3-\beta 3$ \\
\hline D173A & $\downarrow$ & $\downarrow$ & Walker B \\
\hline Y214A & $\downarrow$ & $\downarrow$ & Unresolved loop \\
\hline $\mathrm{N} 215 \mathrm{~A}$ & $\downarrow$ & $\downarrow$ & Unresolved loop \\
\hline $\mathrm{K} 216 \mathrm{~A} / \mathrm{K} 216 \mathrm{E}$ & $V$ & $\downarrow \downarrow$ & Unresolved loop \\
\hline E217Q/E217K/E217A & $V \sqrt{V}$ & $\uparrow \uparrow \uparrow$ & Unresolved loop \\
\hline $\mathrm{D} 218 \mathrm{~A}$ & $V$ & $V$ & Near $\beta 4$ \\
\hline N219A & $\downarrow$ & $\checkmark$ & Near $\beta 4$ \\
\hline P220A & $\nabla$ & $\downarrow$ & $\beta 4$ \\
\hline R293V & $\downarrow$ & $\downarrow$ & Rca-Rca interface \\
\hline K315D & 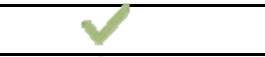 & $\downarrow$ & Helix $9(\alpha 3)$ \\
\hline $\mathrm{R} 321 \mathrm{~A}$ & $\uparrow$ & $\downarrow$ & Surface near $(\alpha 9)$ \\
\hline
\end{tabular}

\begin{tabular}{|c|c|}
\hline & \\
\hline Normal & Slightly reduced \\
\hline & \\
\hline Highly stimulated & Slightly stimulated \\
\hline$\downarrow$ & \\
\hline Strongly reduced & Moderately reduced \\
\hline
\end{tabular}

Table 7.2: Summary of rice activase mutants evaluated in this study 
Taken together the information from the previous study and the data generated in this study, a modified model for Rubisco activation is proposed (Fig. 7.2B). We would like to present a hypothesis, where the newly identified flexible surface loop (red) might directly be involved in Rubisco-Rca interaction as primary site of contact as proposed for helix $9(\alpha 9)$. This loop is also positioned close to the rim of the pore ( 17-22 $\AA$ ), however the mechanism of C-tail pulling through the pore as described for the proteobacterial red-type Rca appears unlikely for plant activase (see 5.3). Therefore, another possibility might be that the new surface loop in conjunction with other surface residues (helix 3) should play an important role in conformational remodelling of the active site by positioning the Rubisco towards central pore for partial threading, leading to release of inhibitor. The distribution of the newly discovered interaction residues suggests that many more protein-protein interactions between Rubisco and activase exist than described so far. The engaged AAA+ ring could be anticipated to bury a significant area of the surface of the Rubisco holoenzyme. 


\section{A}

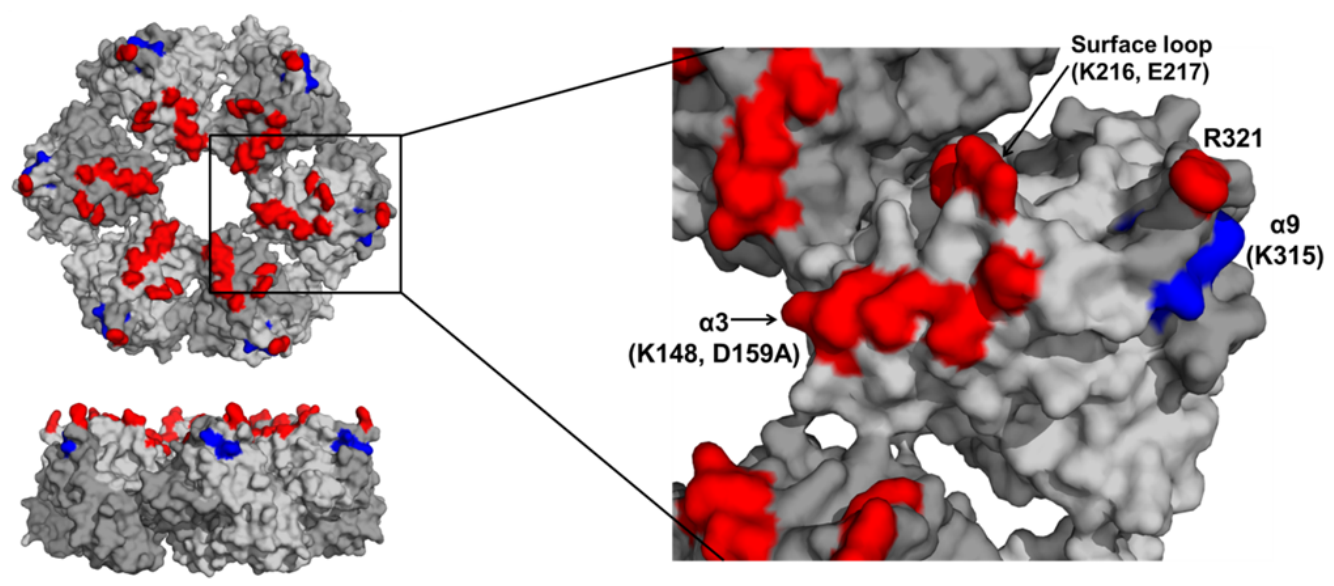

B

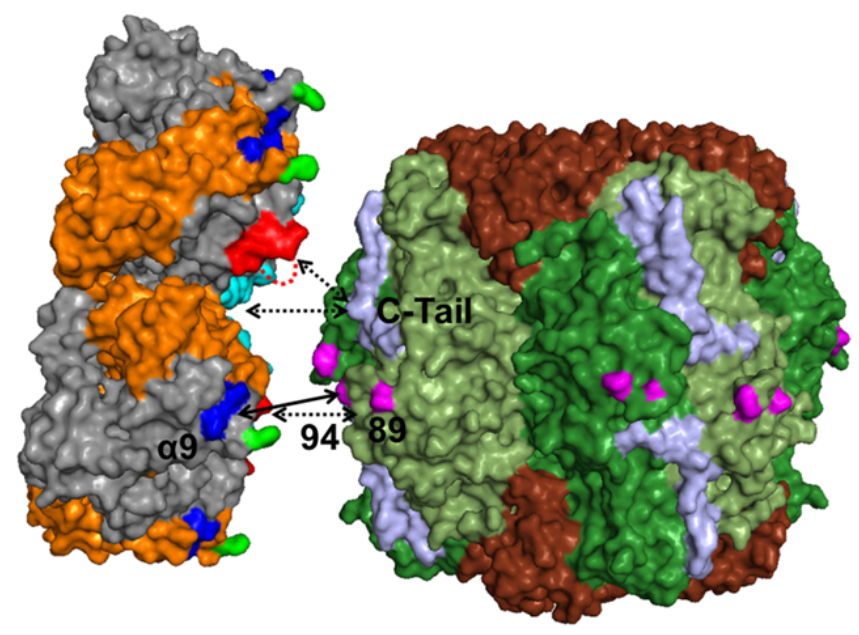

Fig. 7.2: Mechanistic model for Rubisco activation in plant. (A) Surface representation of the six fold symmetry of the Rubisco activase highlighting previously described helix 9 (blue), important for Rubisco interaction. The regions containing newly discovered residues in this study are highlighted in red. (B) Helix $9(\alpha 9$, in blue) and N-terminal domain (not resolved in the crystal structure) might form the initial site of interaction as hypothesised earlier [59, 129]. Residues critical for recognition by Rca are indicated in magenta on Rubisco surface [145]. The flexible surface loop (red) identified in the study might either be involved in Rubisco-Rca interaction as proposed for helix 9 ( $\alpha 9)$ or in remodelling of the active site by affecting the conformation of the C-tail. Helix 3 (cyan) along the pore axis and protruding surface residue (green) might form additional site of contacts for positioning the Rubisco towards central pore for partial threading or bringing about conformational change in Rubisco active site leading to release of inhibitors. This model suggests that Rca involved larger section of the surface element of the Rubisco holoenzyme rather than just C-tail unlike found for RsCbbX in proteobacteria [114]. Surface representation of Rubisco and Rubisco activase from spinach were shown (PDB id: 1RCX and 3ZW6). Rubisco's large subunits were shown in dark and olive green while small subunits were shown in brown. C-tail was displayed in light blue. Rca subunits were shown in grey and orange colour. The solid arrow represents the known Rubisco-Rca interaction site whereas the dotted arrows indicate the hypothesized Rubisco-Rca interaction site. 


\subsection{Biochemical insights into the regulation of Rea by phosphorylation}

Redox regulation by the ferrodoxin-thioredoxin system has already been well established as the mechanism for regulating the activity of Rca under dark/light cycle and variable ADP/ATP ratio [146, 152, 155, 156]. Recently published proteomics data have indicated that in the dark Rca activity could also be regulated by phosphorylation of some residues situated at regions important for Rca function [250, 251].

Mutating the phosphorylated serine to its phosphomimetic counterpart, aspartate, in our study led to normal ATPase rate, while resulting in almost a $80 \%$ loss of Rubisco activation (section 5.4). Thus, this residue appears to be important for Rubisco-Rca interaction. However, its role in inhibiting Rca activity under dark is not conclusive [252]. This is because the phosphorylation pattern is poorly conserved in higher plants. For instance, the phosphorylated threonine in arabidopsis is replaced by isoleucine in rice and maize. On the other hand, in rice, the adjacent serine is phosphorylated, while in maize no phosphorylation was observed [251]. These facts still argue the role for phosphorylation for downregulating photosynthesis by inactivating Rubisco activase in the dark. Conversely, the phosphorylated residue might differ in different species as observed earlier at position, as well in terms of the number of phosphorylation sites. Therefore, it is possible that Rca in different species exhibit different phosphorylating response to modulate its activity under limiting light conditions. Also, unlike redox regulation, the residues for phosphorylation are common in both Rca isoforms and it was found that both the isoforms were evenly phosphorylated at nights [252]. However, any attempt to demonstrate an effect of phosphorylation on Rca activity in the study was not conclusive.

Identifying the phosphorylating targets in other Rca homologues followed by mutagenesis study should provide better insight into regulation process. It is plausible that phosphorylation might possess as important a role as redox regulation in regulating Rca activity. Also, other post translational modifications could be anticipated to be involved in Rubisco regulation and need to be investigated in future. 


\subsection{Distant classes of Rubisco activase have convergently evolved to remodel their respective Rubisco}

Three classes of Rubisco activases have been described so far. The green-type Rca found in higher plants/green algae [9, 129], a red-type Rca (Cbbx) for $\alpha$ proteobacteria/red lineage phytoplankton $[104,114]$ and the recently discovered CbbQO (purple-type Rca) in chemoautotrophic bacteria [115]. All three activases belong to the superfamily of AAA+ proteins and utilise energy of ATP hydrolysis to remodel Rubisco. However, they have poor sequence homology among them and employ different mechanisms of Rubisco activation.

CbbX functions by transiently threading the C-tail peptide of Rubisco large subunits through the central pore of the hexameric ring to remodel Rubisco, similar to widely studied AAA+ homologues including the Hsp100 proteins, ClpB and ClpX [267, 268]. A conserved key tyrosine residue in the pore loop was found to be indispensable in CbbX function, while the pore loop 1 in plant Rca was different to classical pore loop found in $\mathrm{AAA}+$ proteins and shows an absence of tyrosine or aromatic residues. Also, the C-terminus of plant Rca was shorter than that of red-type Rubisco and extending the length of $\mathrm{C}$-terminus in plant does not alter the ability of the enzyme to be activated $[129,269]$. On the other hand, plant Rca interacts with two surface residues on the large subunit of Rubisco via helix 9 residues, additionally involving $\mathrm{N}$-terminal domain as described earlier $[129,145,167,168]$. However, mutational analysis from the previous study [129] and the present study also supports the role of pore loop and surface residues lining the pore loop 1 in Rca remodelling. Additionally, the newly identified surface loop in our study might also implicate itself in the mechanistic function as described in the previous section.

Apart from this, CbbX only forms active hexameric complex in presence of ATP and RuBP and shows stimulatory ATPase rate in presence of substrate Rubisco [114]. Plant Rca was found to be formed hexamer in presence of Mg-ATP or Mg-ATP $\gamma \mathrm{s}$ $[129,141]$ and is constitutively ATPase active without RuBP. Stimulation in presence of its substrate does not occur for plant Rca unlike CbbX [109] and its activity is regulated by $\mathrm{ADP} / \mathrm{ATP}$ ratio and redox state of $\alpha$ isoform. Also, a recently discovered $\mathrm{XuBP}$ phosphatase, called CbbY also functions in cooperation with $\mathrm{CbbX}$ to degrade 
$\mathrm{XuBP}$, arresting its rebinding to Rubisco. The homologous CbbY gene is encoded by all photosynthetic organisms [108].

A

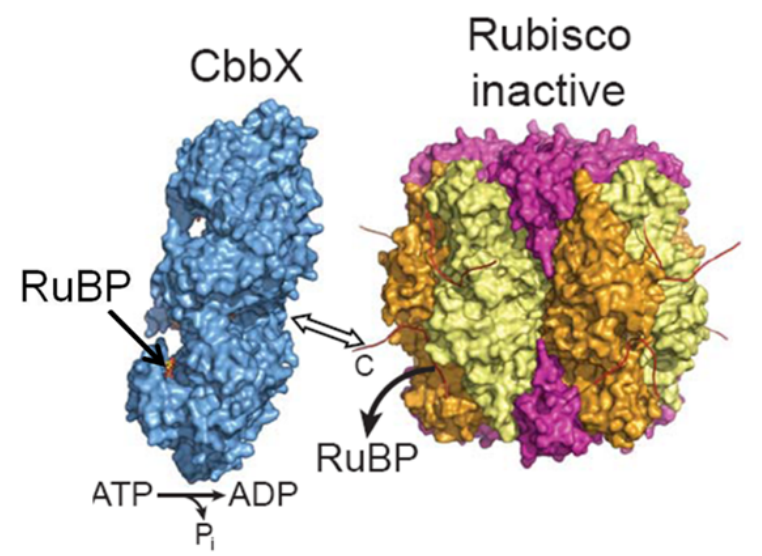

B

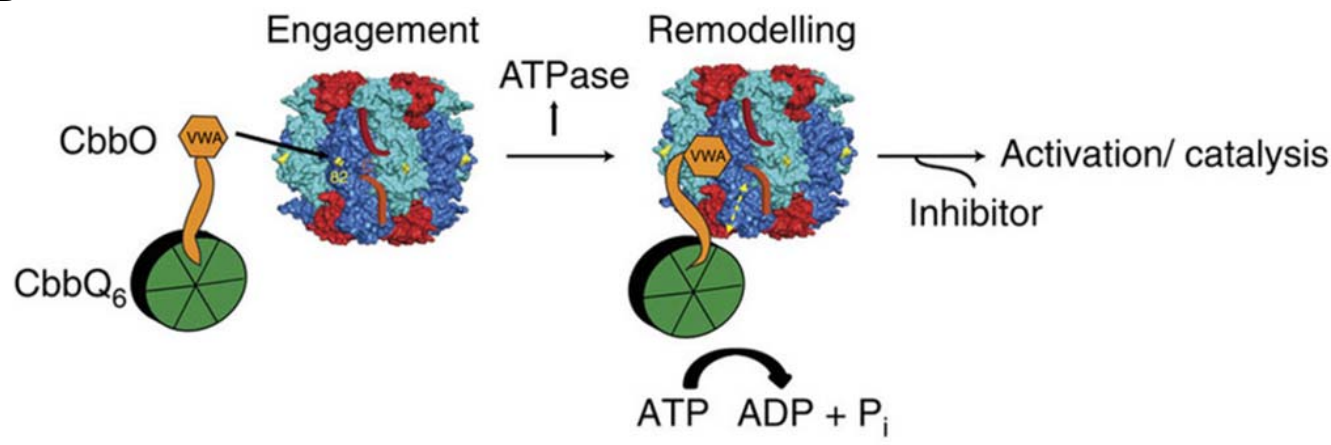

Fig. 7.3: Mechanism of Rubisco activation in different system. (A) Model of Rubisco activation by the Red type Rca, CbbX. The CbbX hexamer docks onto inactive Rubisco and transiently pulls the extended C-terminal sequence of red-type RbcL into the central pore of the hexamer. This action is mediated by the stimulated CbbX ATPase and destabilizes the Rubisco active site, releasing the inhibitory sugar. (B) Model of Rubisco activation mechanism by CbbQO (purple-type) for form I Rubisco. CbbQO binds to inhibited rubisco complexes via the acidic surface residue (Asp 82, coloured yellow) of the rubisco large subunit using the MIDAS binding site located on CbbO. The AAA+ hexamer formed by $\mathrm{CbbQ}$ may also interact with rubisco directly, possibly via the $\mathrm{C}$ terminus of the large subunit (indicated by yellow dashed double arrow). Productive binding, which involves both Asp 82 and the $\mathrm{C}$ terminus, results in a stimulation of ATPase activity. This provides the energy used to remodel the inhibited rubisco active site allowing release of the inhibitor. Adopted from [114, 115]

The newly discovered third type of Rubisco activase system, CbbQO, in chemoautotrophic bacteria deploys a mechanism, whereby the hexameric AAA+ ATPase CbbQ forms a complex with a large protein $\mathrm{CbbO}\left(\mathrm{CbbQ}_{6} \mathrm{O}_{1}\right)$. The later acts as a substrate adaptor for inhibited Rubisco via its von Willebrand Factor A (VWA) domain $[115,270]$. The MIDAS motif of the VWA domain of CbbO interacts with 
the acidic surface residue of the Rubisco (Asp 82). The corresponding surface residue (Pro 89) was found to be crucial for activation of plant Rubisco by Rca [167]. The activation process required necessary involvement of the C-terminus of Rubisco large subunits like CbbX, however, the role of the pore has not been established [115]. The interaction with Rubisco leads to a stimulation of ATPase activity for CbbQ as found for CbbX. The energy produced from the hydrolysis of ATP may be transmitted directionally from CbbQ to the VWA domain through the CbbO linker to disrupt the active site of Rubisco. CbbQO class of Rca does not respond to RuBP, similar to plant Rca, and contrary to CbbX. Thus, the regulation of ATPase by the Calvin-Benson cycle metabolite RuBP appears specific to the red-type activase system for now. Stimulation of ATPase by inhibited substrate Rubisco is observed for the chemoautotrophic CbbQO activases and CbbX but is absent in plant activase systems $[104,109,114,115]$.

Comparing the three activase systems, it could be concluded that the contact points between activase classes are surface residue and the $\mathrm{C}$-terminus, which remain conserved. The secondary structure elements of large subunits of Rubisco are highly homologous [5, 53], however their respective activases demonstrate poor sequence homology among them except the AAA+ core motifs. This suggests that during the course of evolution the activases were recruited from the general AAA+ molecular chaperone machinery in different lineages and are hence a product of convergent evolution. It appears likely that additional convergently evolved Rca systems are yet to be discovered.

\subsection{Conclusions and future perspectives}

Considering the problem of population explosion and climate change there is an obligate need to take measures to ensure food security for the coming generations. Optimization of photosynthetic carbon fixation has been the current focus, since the benefits gained from the previous approaches have already reached their saturation limit $[2,15]$. Rubisco being the key enzyme of photosynthesis has always been a potential target for improvement in terms of its catalytic efficiency. However, this approach suffers from a variety of limitations owing to complexities involved in recombinant expression of more efficient algal Rubisco in the higher plant chloroplast due to chaperone compatibility issues $[49,271]$. However, success is anticipated in 
resolving these issues due to rapid advancements in understanding the factors involved in assembly and biogenesis of green-type Rubisco [59, 63, 272, 273].

Improving just Rubisco might not solve the problem as Rubisco is highly prone to inhibition by sugar phosphates and requires its helper protein, Rubisco activase, to dissociate them from the active site. Although Rubisco is quite thermostable at higher temperature, photosynthesis ceases at moderately high temperatures [174]. This thermal intolerance of photosynthesis is believed to be attributed to the thermolability of plant Rubisco activase which gets denatured and fails to maintain the activation state of Rubisco under heat stress $[10,11,169]$. To extract full benefits for enhancing photosynthetic efficiency, Rubisco manipulation strategies should be co-integrated with activase engineering strategies. Addressing the issue pertaining to thermal stability of activase should provide preparatory measures to deal with the problem associated with the future climatic changes $[12,18]$.

Our work focussed on the biochemical characterization of the rice Rubisco activase to develop a more detailed mechanistic appreciation of the Rubisco activation system. In parallel, we have also discovered and presented a detailed characterization of the highly thermostable and effective Rca from the CAM plant, Agave tequilana. This system was later utilised to engineer thermostable rice-agave activase chimeras. The in vitro biochemical data suggests that such thermostable activases could be introduced into host plants in vivo directly or as chimera without any compatibility issue. If so, this might address the problem of thermolability associated with most of the plant activases and improve functionality at elevated temperature.

Attempts to exactly locate residues or motifs responsible for thermostability in the study were not successful, as the point mutations tested in this study did not yield informative results. A future approach should be to target small domains or motifs for mutagenesis. Nevertheless, the knowledge obtain through our result might be crucial for engineering not only thermostable but also functionally more effective activase variants of rice and other crops.

The mutational studies performed in this thesis have provided an additional layer of information towards the mechanistic understanding of the Rubisco activation process. The interesting behaviour of some mutants at different locations in the structure 
requires further follow up and will serve as framework for future studies. A major milestone in plant Rubisco activase research would be the establishment of conditions that favour a stable Rca-Rubisco complex. Structural and biochemical characterization of such a complex would then allow rapid progress to be made in our understanding of the activation process. There is some evidence for trap-mutants in other AAA+ systems [239] and a complex between Rubisco and its assembly chaperone $\mathrm{RbcX}$ has been obtained [274]. Also the crosslinking experiments with the red-type Rca of Rhodobacter sphaeroides have been successful (unpublished results from Oliver Mueller-Cajar), but as of now there is no solid evidence of a stable plant Rca-Rubisco complex, likely due to the transient nature of the interaction between them. Discovery of high affinity activase-Rubisco combination as shown here for agave-rice will be useful in this context. Additionally, other biophysical techniques such as H/D exchange by mass spectrometry should be attempted to identify residues involved in Rubisco-Rca interaction. Furthermore, structural characterization of the intact N-terminal domain which is presumed to be an adaptor for recruitment of Rubisco would be useful to validate its role.

We have successfully demonstrated the formation of covalent complex between the $\alpha$ Rca of rice and a thioredoxin-f mutant. Attempt should be made to purify such a complex to homogeneity for biochemical and structural studies. In the absence of structural information the mechanism of redox regulation is not well understood. Obtaining a structure of Rca in oxidised and reduced state would aid in a detailed understanding of activase regulation.

A major obstacle in interrogating protein-protein interactions between Rubisco and Rubisco activase is our inability to produce recombinant plant Rubisco in E.coli or other microbial hosts $[275,276]$. This limits our ability to generate Rubisco mutants, which could allow us to test hypotheses on activase function by modifying substrate protein. The prokaryotic Rubiscos that are closely related to plants are recombinantly produced as part of parallel projects in our lab and are amenable to extensive mutagenesis to make them compatible. A favourable outcome would be if such Rubiscos could be activated by plant activases. The directed evolution study using artificial evolution systems should facilitate this outcome in future [276] (unpublished data of Zhijun Guo). Successful advances in such an approach would greatly expand 
our ability to further test our hypothesis concerning the mechanism of activation as successfully done in other activase classes [114, 115]. Beside this, a co-variation study of multiple Rca and Rubisco sequences should be performed using coevolution-based computational tools [277-279] to predict contact points between the two proteins, which could be further verified experimentally.

In summary, this study generated a comprehensive molecular platform to address pertinent questions in Rubisco regulation and maintenance from biochemical angle. Our results indicate that further bioprospecting and careful biochemical characterization of Rca homologues will lead to both mechanistic insights and identification of candidate proteins for engineering enhanced thermotolerance in rice and other crops. Such genetically modified variants of crops are expected to show better adoptability in the predicted future climatic conditions, eventually leading to enhanced agricultural productivity. The importance of such work is difficult to overstate, considering the significant sustainability challenges encroaching on our species in the coming decades. We hope our work will inform that of others who are similarly approaching the same problem from multiple angles [280-282]. 


\section{Appendix}


A

Atig73110262377_at

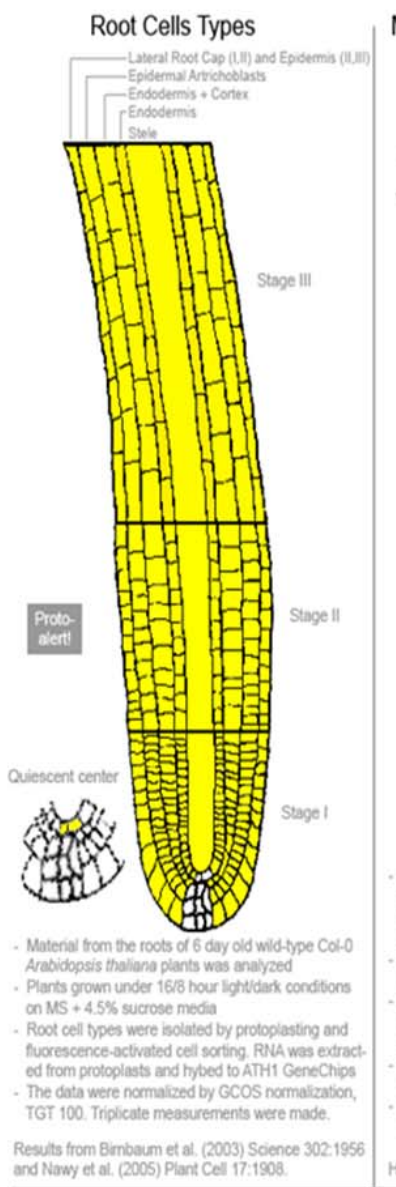

Microgametogenesis (Pollen Development)
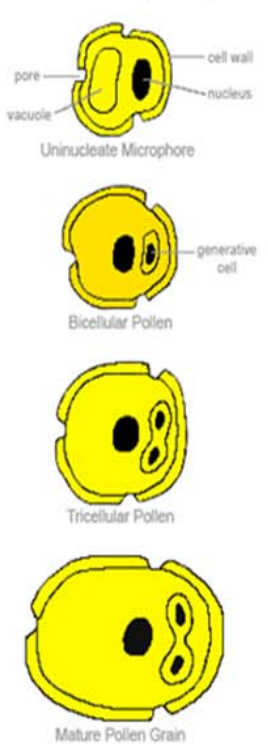

Plant material from the polien of 5.10 growth stage wid-type Arabicopsis thaliana plants of Ler-S ecotype was anayzed Plants gromn under 1618 hou Ilitudark condisons at $21^{\circ} \mathrm{C}$ doplone the swere of duplcates - the average of Mich is shown

The ATH Cenechip hybridzed to Re Aithi Genechip The diata were namalsed by GCOS
Embryo Development
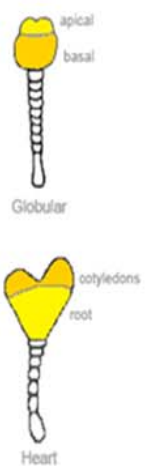

$\cos$

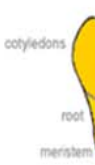

Arabidopsis eFP Browser at bar.utoronto.ca Winter et al. 2007. PLoS One 2(8): e718 Xylem and Cork

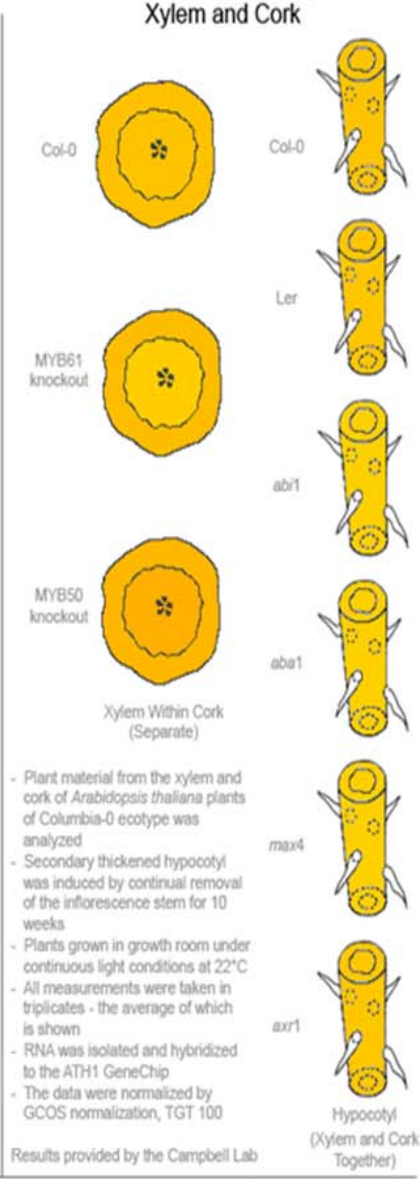


B

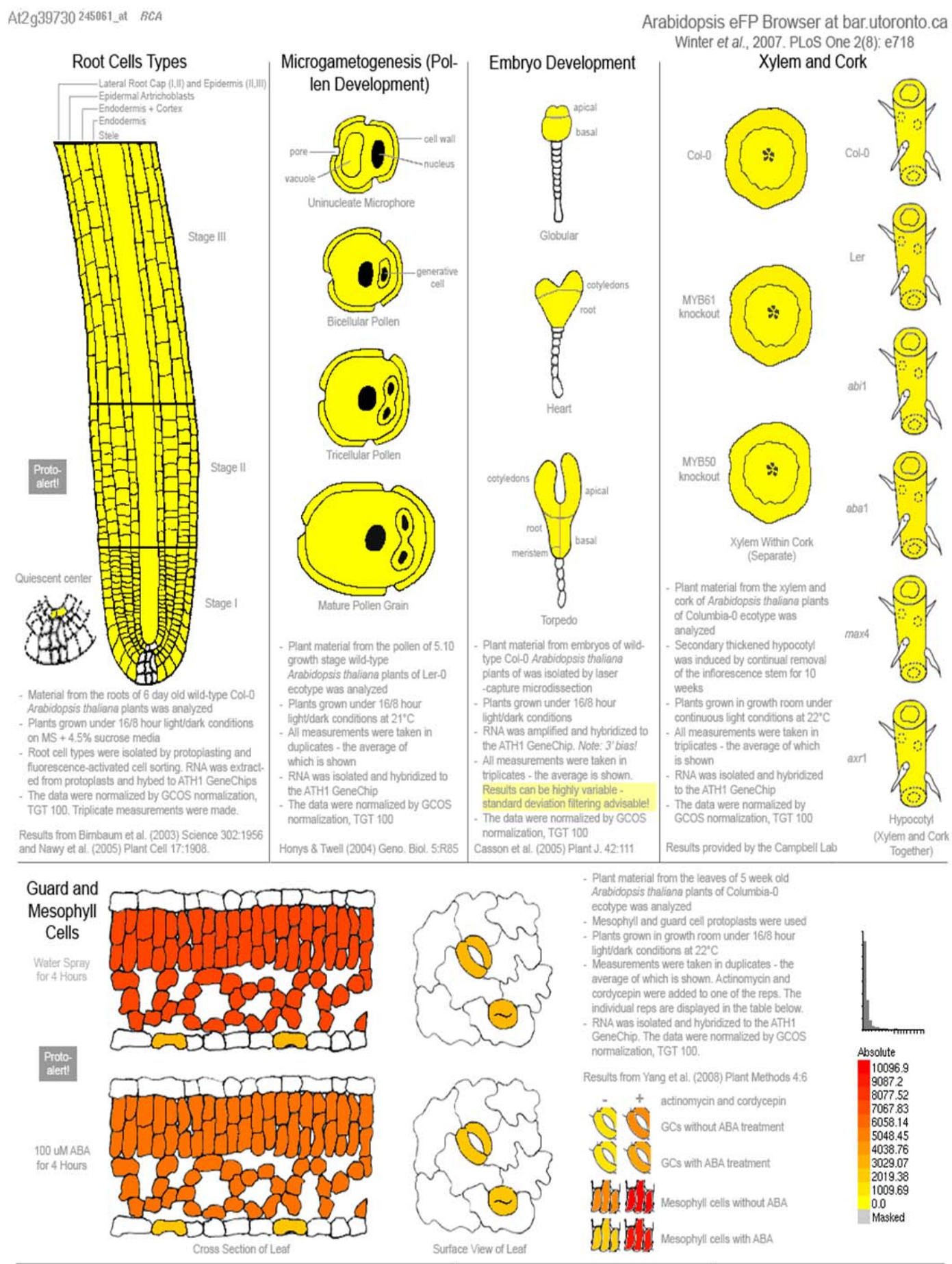

Fig. 8.1: Tissue specific expression level of Rca2 (A) and Rubisco activase (B) as obtained from The arabidopsis eFP browser [256]. 


\begin{tabular}{|l|l|l|}
\hline Organism & Rca2 & Rca \\
\hline Agave tequilana & Locus 30142 & Locus 27298 \\
\hline Arabidopsis thaliana & Q9AST9 & P10896 \\
\hline Cajanus cajan & A0A151S6K1 & A0A151R088 \\
\hline Capsella rubella & R0IAN8 & R0HC86 \\
\hline Chlamydomonas reinhardtii & A8JJ0 & P23489 \\
\hline Chlorella variabilis & E1ZCY8 & E1Z378 \\
\hline Cucumis sativus & A0A0A0K6Q9 & Q01587 \\
\hline Daucus carota & A0A164U7N2 & A0A166DCY4 \\
\hline Glycine max & I1M608 & D4N5G0 \\
\hline Gossypium arboreum & XP_017603330.1 & A0A0B0Q068 \\
\hline Micromonas commoda & C1EGP0 & C1E4S4 \\
\hline Musa acuminata & M0RMV9 & M0RV12 \\
\hline Oryza sativa & Q7XN85 & P93431 \\
\hline Ostreococcus lucimarinus & A4RW51 & A4RW20 \\
\hline Phaseolus vulgaris & V7BY25 & O64981 \\
\hline Physcomitrella patens & A9TYZ9 & A9TBP0 \\
\hline Ricinus communis & B9RGR9 & B9SDY7 \\
\hline Solanum lycopersicum & K4BLT6 & K4D489 \\
\hline Spinacia oleracea & A0A0K9QK09 & P10871 \\
\hline Triticum aestivum & W5BC60 & Q9M4V3 \\
\hline Vigna radiata & XP_014501031.1 & O98997 \\
\hline Zea mays & A0A144KR40 & COP5X1 \\
\hline
\end{tabular}

Table 8.1: List of species and accession numbers for Rca2 and Rca sequences from plants and green algae used for the phylogenetic analysis. 


\section{References}


1. Gornall, J., et al., Implications of climate change for agricultural productivity in the early twenty-first century. Philosophical Transactions of the Royal Society of London B: Biological Sciences, 2010. 365(1554): p. 2973-2989.

2. Zhu, X.G., S.P. Long, and D.R. Ort, Improving photosynthetic efficiency for greater yield. Annual Review of Plant Biology, 2010. 61: p. 235-61.

3. Ort, D.R., et al., Redesigning photosynthesis to sustainably meet global food and bioenergy demand. Proceedings of the National Academy of Sciences of the United States of America, 2015. 112(28): p. 8529-8536.

4. Whitney, S.M., R.L. Houtz, and H. Alonso, Advancing our understanding and capacity to engineer nature's CO2-sequestering enzyme, Rubisco. Plant Physiology, 2011. 155(1): p. $27-$ 35.

5. Andersson, I. and A. Backlund, Structure and function of Rubisco. Plant Physiology and Biochemistry, 2008. 46(3): p. 275-291.

6. Bauwe, H., M. Hagemann, and A.R. Fernie, Photorespiration: players, partners and origin. Trends in Plant Science, 2010. 15(6): p. 330-336.

7. Parry, M.A., et al., Rubisco regulation: a role for inhibitors. Journal of Experimental Botany, 2008. 59(7): p. 1569-80.

8. Portis, A.R., Rubisco activase - Rubisco's catalytic chaperone. Photosynthesis Research, 2003. 75(1): p. 11-27.

9. Salvucci, M.E., A.R. Portis, Jr., and W.L. Ogren, A soluble chloroplast protein catalyzes ribulosebisphosphate carboxylase/oxygenase activation in vivo. Photosynthesis Research, 1985. 7(2): p. 193-201.

10. Salvucci, M.E. and S.J. Crafts-Brandner, Inhibition of photosynthesis by heat stress: the activation state of Rubisco as a limiting factor in photosynthesis. Physiologia Plantarum, 2004. 120(2): p. 179-186.

11. Crafts-Brandner, S.J. and M.E. Salvucci, Rubisco activase constrains the photosynthetic potential of leaves at high temperature and CO2. Proceedings of the National Academy of Sciences of the United States of America, 2000. 97(24): p. 13430-13435.

12. Kurek, I., et al., Enhanced thermostability of arabidopsis rubisco activase improves photosynthesis and growth rates under moderate heat stress. The Plant Cell, 2007. 19(10): p. $3230-41$

13. Kumar, A., C. Li, and A.R. Portis, Jr., Arabidopsis thaliana expressing a thermostable chimeric Rubisco activase exhibits enhanced growth and higher rates of photosynthesis at moderately high temperatures. Photosynthesis Research, 2009. 100(3): p. 143-53.

14. Carmo-Silva, E., et al., Optimizing Rubisco and its regulation for greater resource use efficiency. Plant, Cell \& Environment, 2015. 38(9): p. 1817-1832.

15. Parry, M.A., et al., Rubisco activity and regulation as targets for crop improvement. Journal of Experimental Botany, 2013. 64(3): p. 717-30.

16. Sharkey, T.D., Effects of moderate heat stress on photosynthesis: importance of thylakoid reactions, rubisco deactivation, reactive oxygen species, and thermotolerance provided by isoprene. Plant, Cell \& Environment, 2005. 28(3): p. 269-277.

17. Sage, R.F. and D.S. Kubien, The temperature response of C(3) and C(4) photosynthesis. Plant, Cell \& Environment, 2007. 30(9): p. 1086-106. 
18. Sage, R.F., D.A. Way, and D.S. Kubien, Rubisco, Rubisco activase, and global climate change. Journal of Experimental Botany, 2008. 59(7): p. 1581-95.

19. Nelson, D.L., A.L. Lehninger, and M.M. Cox, Lehninger Principles of Biochemistry. 2008: Macmillan.

20. Soll, J. and E. Schleiff, Protein import into chloroplasts. Nature Reviews Molecular Cell Biology, 2004. 5(3): p. 198-208.

21. Campbell, N. and J. Reece, Biology. 7th. Ed Pearson Benjamin Cummings. Cape Town, 2005.

22. Merchant, S. and M.R. Sawaya, The light reactions: a guide to recent acquisitions for the picture gallery. The Plant Cell, 2005. 17(3): p. 648-63.

23. Cruz, J.A., et al., Plasticity in light reactions of photosynthesis for energy production and photoprotection. Journal of Experimental Botany, 2005. 56(411): p. 395-406.

24. Leslie, M., On the origin of photosynthesis. Science, 2009. 323(5919): p. 1286-1287.

25. Benson, A.A., Following the path of carbon in photosynthesis: a personal story. Photosynthesis Research, 2002. 73(1): p. 29.

26. Ellis, R.J., The most abundant protein in the world. Trends in Biochemical Sciences, 1979. 4(11): p. 241-244.

27. Raven, J.A., Rubisco: still the most abundant protein of Earth? New Phytologist, 2013. 198(1): p. 1-3.

28. Shively, J.M., G. van Keulen, and W.G. Meijer, Something from almost nothing: carbon dioxide fixation in chemoautotrophs. Annual Review of Microbiology, 1998. 52: p. 191-230.

29. Spreitzer, R.J. and M.E. Salvucci, Rubisco: structure, regulatory interactions, and possibilities for a better enzyme. Annual Review of Plant Biology, 2002. 53: p. 449-75.

30. Linster, C.L., E. Van Schaftingen, and A.D. Hanson, Metabolite damage and its repair or preemption. Nature Chemical Biology, 2013. 9(2): p. 72-80.

31. Ainsworth, E.A. and S.P. Long, What have we learned from 15 years of free-air $\mathrm{CO}_{2}$ enrichment (FACE)? A meta-analytic review of the responses of photosynthesis, canopy properties and plant production to rising $\mathrm{CO}_{2}$. New Phytologist, 2005. 165(2): p. 351-372.

32. Drake, B.G., M.A. Gonzalez-Meler, and S.P. Long, More efficient plants: A consequence of rising atmospheric $\mathrm{CO}_{2}$ ? Annual Review of Plant Physiology and Plant Molecular Biology, 1997. 48: p. 609-639.

33. Long, S.P., et al., Food for thought: Lower-than-expected crop yield stimulation with rising $\mathrm{CO}_{2}$ concentrations. Science, 2006. 312(5782): p. 1918-1921.

34. Kebeish, R., et al., Chloroplastic photorespiratory bypass increases photosynthesis and biomass production in Arabidopsis thaliana. Nature Biotechnology, 2007. 25(5): p. 593-9.

35. Maier, A., et al., Transgenic introduction of a glycolate oxidative cycle into A. thaliana chloroplasts leads to growth improvement. Frontiers in Plant Science, 2012. 3: p. 38.

36. Taler, D., et al., Plant eR genes that encode photorespiratory enzymes confer resistance against disease. The Plant Cell, 2004. 16(1): p. 172-84.

37. Bloom, A.J., et al., Carbon dioxide enrichment inhibits nitrate assimilation in wheat and Arabidopsis. Science, 2010. 328(5980): p. 899-903.

38. Price, G.D., et al., Advances in understanding the cyanobacterial CO2-concentratingmechanism (CCM): functional components, Ci transporters, diversity, genetic regulation and 
prospects for engineering into plants. Journal of Experimental Botany, 2008. 59(7): p. 144161.

39. Bonacci, W., et al., Modularity of a carbon-fixing protein organelle. Proceedings of the National Academy of Sciences of the United States of America, 2012. 109(2): p. 478-83.

40. Moroney, J.V. and R.A. Ynalvez, Proposed carbon dioxide concentrating mechanism in Chlamydomonas reinhardtii. Eukaryotic Cell, 2007. 6(8): p. 1251-9.

41. Badger, M.R., et al., The diversity and coevolution of Rubisco, plastids, pyrenoids, and chloroplast-based $\mathrm{CO}_{2}$-concentrating mechanisms in algae. Canadian Journal of Botany, 1998. 76(6): p. 1052-1071.

42. Keeley, J.E. and P.W. Rundel, Evolution of CAM and C4 carbon-concentrating mechanisms. International Journal of Plant Sciences, 2003. 164(S3): p. S55-S77.

43. Kajala, K., et al., Strategies for engineering a two-celled C(4) photosynthetic pathway into rice. Journal of Experimental Botany, 2011. 62(9): p. 3001-10.

44. von Caemmerer, S., W.P. Quick, and R.T. Furbank, The development of C(4)rice: current progress and future challenges. Science, 2012. 336(6089): p. 1671-2.

45. Dodd, A.N., et al., Crassulacean acid metabolism: plastic, fantastic. Journal of Experimental Botany, 2002. 53(369): p. 569-80.

46. Edmondson, D.L., H.J. Kane, and T.J. Andrews, Substrate isomerization inhibits ribulosebisphospate carboxylase-oxygenase during catalysis. FEBS Letters, 1990. 260(1): p. 62-66.

47. Mueller-Cajar, O., M. Stotz, and A. Bracher, Maintaining photosynthetic $\mathrm{CO}_{2}$ fixation via protein remodelling: the Rubisco activases. Photosynthesis Research, 2014. 119(1): p. 191201.

48. Parry, M., et al., Prospects for increasing photosynthesis by overcoming the limitations of Rubisco. Journal of Agricultural Science-Cambridge-, 2007. 145(1): p. 31.

49. Whitney, S.M., et al., Form I Rubiscos from non-green algae are expressed abundantly but not assembled in tobacco chloroplasts. Plant Journal, 2001. 26(5): p. 535-547.

50. Uemura, K., S. Miyachi, and A. Yokota, Ribulose-1, 5-bisphosphate carboxylase/oxygenase from thermophilic red algae with a strong specificity for $\mathrm{CO}_{2}$ fixation. Biochemical and Biophysical Research Communications, 1997. 233(2): p. 568-571.

51. Read, B.A. and F.R. Tabita, High substrate specificity factor ribulose bisphosphate carboxylase/oxygenase from eukaryotic marine algae and properties of recombinant cyanobacterial RubiSCO containing "algal" residue modifications. Archives of Biochemistry and Biophysics, 1994. 312(1): p. 210-8.

52. Tabita, F.R., Microbial ribulose 1,5-bisphosphate carboxylaseloxygenase: A different perspective. Photosynthesis Research, 1999. 60(1): p. 1-28.

53. Tabita, F.R., et al., Distinct form I, II, III, and IV Rubisco proteins from the three kingdoms of life provide clues about Rubisco evolution and structure/function relationships. Journal of Experimental Botany, 2008. 59(7): p. 1515-1524.

54. Badger, M.R. and E.J. Bek, Multiple Rubisco forms in proteobacteria: their functional significance in relation to $\mathrm{CO}_{2}$ acquisition by the $\mathrm{CBB}$ cycle. Journal of Experimental Botany, 2008. 59(7): p. 1525-1541.

55. Tabita, F.R., et al., Function, structure, and evolution of the RubisCO-like proteins and their RubisCO homologs. Microbiology and Molecular Biology Reviews, 2007. 71(4): p. 576-599. 
56. Li, H., et al., Crystal structure of a RuBisCO-like protein from the green sulfur bacterium Chlorobium tepidum. Structure, 2005. 13(5): p. 779-89.

57. Blair, G.E. and R.J. Ellis, Protein-synthesis in chloroplasts .1. Light-driven synthesis of large subunit of fraction I protein by isolated pea chloroplasts. Biochimica et Biophysica Acta, 1973. 319(2): p. 223-234.

58. Hartman, F.C. and M.R. Harpel, Structure, function, regulation, and assembly of D-ribulose1,5-bisphosphatecarboxylase oxygenase. Annual Review of Biochemistry, 1994. 63: p. 197234.

59. Hauser, T., et al., Role of auxiliary proteins in Rubisco biogenesis and function. Nature Plants, 2015. 1(6).

60. Spreitzer, R.J., S.R. Peddi, and S. Satagopan, Phylogenetic engineering at an interface between large and small subunits imparts land-plant kinetic properties to algal Rubisco. Proceedings of the National Academy of Sciences of the United States of America, 2005. 102(47): p. 17225-17230.

61. Spreitzer, R.J., Role of the small subunit in ribulose-1,5-bisphosphate carboxylase/oxygenase. Archives of Biochemistry and Biophysics, 2003. 414(2): p. 141-149.

62. Ishikawa, C., et al., Functional incorporation of sorghum small subunit increases the catalytic turnover rate of Rubisco in transgenic rice. Plant Physiology, 2011. 156(3): p. 1603-1611.

63. Joshi, J., et al., Role of small subunit in mediating assembly of red-type form I rubisco. Journal of Biological Chemistry, 2015. 290(2): p. 1066-1074.

64. Tabita, F.R., et al., Phylogenetic and evolutionary relationships of RubisCO and the RubisCOlike proteins and the functional lessons provided by diverse molecular forms. Philosophical Transactions of the Royal Society B: Biological Sciences, 2008. 363(1504): p. 2629.

65. Sato, T., H. Atomi, and T. Imanaka, Archaeal type III RuBisCOs function in a pathway for AMP metabolism. Science, 2007. 315(5814): p. 1003-1006.

66. Kitano, K., et al., Crystal structure of a novel-type archaeal Rubisco with pentagonal symmetry. Structure, 2001. 9(6): p. 473-481.

67. Maeda, N., et al., Ribulose bisphosphate carboxylase/oxygenase from the hyperthermophilic archaeon Pyrococcus kodakaraensis KOD1 is composed solely of large subunits and forms a pentagonal structure. Journal of Molecular Biology, 1999. 293(1): p. 57-66.

68. Ezaki, S., et al., Presence of a structurally novel type ribulose-bisphosphate carboxylase/oxygenase in the hyperthermophilic archaeon, Pyrococcus kodakaraensis KOD1. Journal of Biological Chemistry, 1999. 274(8): p. 5078-5082.

69. Watson, G.M.F., J.P. Yu, and F.R. Tabita, Unusual ribulose 1,5-bisphosphate carboxylase/oxygenase of anoxic Archaea. Journal of Bacteriology, 1999. 181(5): p. 15691575 .

70. Alonso, H., et al., Substrate-induced assembly of Methanococcoides burtonii D-ribulose-1,5bisphosphate carboxylase/oxygenase dimers into decamers. Journal of Biological Chemistry, 2009. 284(49): p. 33876-82.

71. Imker, H.J., et al., Mechanistic diversity in the RuBisCO superfamily: The "Enolase" in the methionine salvage pathway in Geobacillus kaustophilus. Biochemistry, 2007. 46(13): p. 4077-4089.

72. Hanson, T.E. and F.R. Tabita, A ribulose-1, 5-bisphosphate carboxylase/oxygenase (RubisCO)-like protein from Chlorobium tepidum that is involved with sulfur metabolism and the response to oxidative stress. Proceedings of the National Academy of Sciences of the United States of America, 2001. 98(8): p. 4397-4402. 
73. Ashida, H., et al., A functional link between RuBisCO-like protein of Bacillus and photosynthetic RuBisCO. Science, 2003. 302(5643): p. 286-290.

74. Lorimer, G.H., M.R. Badger, and T.J. Andrews, The activation of ribulose-1, 5-bisphosphate carboxylase by carbon dioxide and magnesium ions. Equilibria, kinetics, a suggested mechanism, and physiological implications. Biochemistry, 1976. 15(3): p. 529-536.

75. Schneider, G., et al., 3-Dimensional Structure of Ribulose-1,5-Bisphosphate CarboxylaseOxygenase from Rhodospirillum-Rubrum at 2.9 a Resolution. The EMBO Journal, 1986. 5(13): p. 3409-3415.

76. Cleland, W.W., et al., Mechanism of Rubisco: The carbamate as general base. Chemical Reviews, 1998. 98(2): p. 549-561.

77. Schreuder, H.A., et al., Crystal structure of activated tobacco rubisco complexed with the reaction-intermediate analogue 2-carboxy-arabinitol 1, 5-bisphosphate. Protein Science, 1993. 2(7): p. 1136-1146.

78. Taylor, T.C. and I. Andersson, Structural transitions during activation and ligand binding in hexadecameric Rubisco inferred from the crystal structure of the activated unliganded spinach enzyme. Nature Structural \& Molecular Biology, 1996. 3(1): p. 95-101.

79. Duff, A.P., T.J. Andrews, and P.M.G. Curmi, The transition between the open and closed states of rubisco is triggered by the inter-phosphate distance of the bound bisphosphate. Journal of Molecular Biology, 2000. 298(5): p. 903-916.

80. Andrews, T.J. and G.H. Lorimer, Rubisco: structure, mechanisms and prospects for improvement. The Biochemistry of Plants, 1987. 10: p. 131-218.

81. Andrews, T.J., The bait in the Rubisco mousetrap. Nature Structural \& Molecular Biology, 1996. 3(1): p. 3-7.

82. Pearce, F.G., Catalytic by-product formation and ligand binding by ribulose bisphosphate carboxylases from different phylogenies. Biochemical Journal, 2006. 399(3): p. 525-534.

83. Jordan, D.B. and R. Chollet, Inhibition of ribulose bisphosphate carboxylase by substrate ribulose 1, 5-bisphosphate. Journal of Biological Chemistry, 1983. 258(22): p. 13752-13758.

84. Berry, J.A., et al., Isolation, identification, and synthesis of 2-carboxyarabinitol 1-phosphate, a diurnal regulator of ribulose-bisphosphate carboxylase activity. Proceedings of the National Academy of Sciences of the United States of America, 1987. 84(3): p. 734-738.

85. Andralojc, P.J., et al., 2-Carboxy-D-arabinitol 1-phosphate (CA1P) phosphatase: evidence for a wider role in plant Rubisco regulation. Biochemical Journal, 2012. 442: p. 733-742.

86. McNevin, D., S. Von Caemmerer, and G. Farquhar, Determining RuBisCO activation kinetics and other rate and equilibrium constants by simultaneous multiple non-linear regression of a kinetic model. Journal of Experimental Botany, 2006. 57(14): p. 3883-3900.

87. Jordan, D.B., R. Chollet, and W.L. Ogren, Binding of phosphorylated effectors by active and inactive forms of ribulose 1, 5-bisphosphate carboxylase. Biochemistry, 1983. 22(14): p. 3410-3418.

88. Pierce, J., N. Tolbert, and R. Barker, Interaction of ribulosebisphosphate carboxylase/oxygenase with transition-state analogs. Biochemistry, 1980. 19(5): p. 934-942.

89. Seemann, J.R., et al., Regulation of ribulose bisphosphate carboxylase activity in vivo by a light-modulated inhibitor of catalysis. Proceedings of the National Academy of Sciences of the United States of America, 1985. 82(23): p. 8024-8028. 
90. Knight, S., I. Andersson, and C.-I. Brändén, Crystallographic analysis of ribulose 1, 5bisphosphate carboxylase from spinach at 2.4 A resolution: Subunit interactions and active site. Journal of Molecular Biology, 1990. 215(1): p. 113-160.

91. Newman, J. and S. Gutteridge, Structure of an effector-induced inactivated state of ribulose 1, 5-bisphosphate carboxylase/oxygenase: the binary complex between enzyme and xylulose 1, 5-bisphosphate. Structure, 1994. 2(6): p. 495-502.

92. Schloss, J., Comparative affinities of the epimeric reaction-intermediate analogs 2-and 4carboxy-D-arabinitol 1, 5-bisphosphate for spinach ribulose 1, 5-bisphosphate carboxylase. Journal of Biological Chemistry, 1988. 263(9): p. 4145-4150.

93. Andrews, T. and M. Hatch, Activity and properties of ribulosediphosphate carboxylase from plants with the C 4-dicarboxylic acid pathway of photosynthesis. Phytochemistry, 1971. 10(1): p. 9-15.

94. Edmondson, D.L., M.R. Badger, and T.J. Andrews, Slow inactivation of ribulosebisphosphate carboxylase during catalysis is caused by accumulation of a slow, tight-binding inhibitor at the catalytic site. Plant Physiology, 1990. 93(4): p. 1390-1397.

95. Robinson, S.P. and A.R. Portis, Ribulose-1, 5-bisphosphate carboxylase/oxygenase activase protein prevents the in vitro decline in activity of ribulose-1, 5-bisphosphate carboxylase/oxygenase. Plant Physiology, 1989. 90(3): p. 968-971.

96. Zhu, G. and R.G. Jensen, Xylulose 1, 5-bisphosphate synthesized by ribulose 1, 5bisphosphate carboxylase/oxygenase during catalysis binds to decarbamylated enzyme. Plant Physiology, 1991. 97(4): p. 1348-1353.

97. Paech, C., et al., Inhibition of ribulose-1, 5-bisphosphate carboxylase/oxygenase by ribulose1, 5-bisphosphate epimerization and degradation products. Biochemical and Biophysical Research Communications, 1978. 83(3): p. 1084-1092.

98. Zhu, G., et al., Formation of the tight-binding inhibitor, 3-ketoarabinitol-1, 5-bisphosphate by ribulose-1, 5-bisphosphate carboxylase/oxygenase is $\mathrm{O}_{2}$-dependent. Photosynthesis Research, 1998. 55(1): p. 67-74.

99. Schrader, S.M., et al., High temperature enhances inhibitor production but reduces fallover in tobacco Rubisco. Functional Plant Biology, 2006. 33(10): p. 921-929.

100. Pearce, F.G. and T.J. Andrews, The relationship between side reactions and slow inhibition of ribulose-bisphosphate carboxylase revealed by a loop 6 mutant of the tobacco enzyme. Journal of Biological Chemistry, 2003. 278(35): p. 32526-32536.

101. Uemura, K., et al., Distribution of fallover in the carboxylase reaction and fallover-inducible sites among ribulose 1, 5-bisphosphate carboxylase/oxygenases of photosynthetic organisms. Plant and Cell Physiology, 1998. 39(2): p. 212-219.

102. Zhu, X.G., A. Portis, and S. Long, Would transformation of C3 crop plants with foreign Rubisco increase productivity? A computational analysis extrapolating from kinetic properties to canopy photosynthesis. Plant, Cell \& Environment, 2004. 27(2): p. 155-165.

103. Tcherkez, G.G., G.D. Farquhar, and T.J. Andrews, Despite slow catalysis and confused substrate specificity, all ribulose bisphosphate carboxylases may be nearly perfectly optimized. Proceedings of the National Academy of Sciences of the United States of America, 2006. 103(19): p. 7246-7251.

104. Loganathan, N., Y.-C.C. Tsai, and O. Mueller-Cajar, Characterization of the heterooligomeric red-type rubisco activase from red algae. Proceedings of the National Academy of Sciences of the United States of America, 2016. 113(49): p. 14019-14024. 
105. Andrews, T.J. and K.M. Abel, Kinetics and subunit interactions of ribulose bisphosphate carboxylase-oxygenase from the cyanobacterium, Synechococcus sp. Journal of Biological Chemistry, 1981. 256(16): p. 8445-51.

106. Lee, B.G., B.A. Read, and F.R. Tabita, Catalytic properties of recombinant octameric, hexadecameric, and heterologous cyanobacterial/bacterial ribulose- 1,5-bisphosphate carboxylase/oxygenase. Archives of Biochemistry and Biophysics, 1991. 291(2): p. 263-9.

107. Robinson, S.P., et al., Purification and assay of Rubisco activase from leaves. Plant Physiology, 1988. 88(4): p. 1008-1014.

108. Bracher, A., et al., Degradation of potent Rubisco inhibitor by selective sugar phosphatase. Nature Plants, 2015. 1(1).

109. Robinson, S.P. and A.R. Portis, Jr., Adenosine triphosphate hydrolysis by purified rubisco activase. Archives of Biochemistry and Biophysics, 1989. 268(1): p. 93-9.

110. Somerville, C.R., A.R. Portis, and W.L. Ogren, A mutant of Arabidopsis thaliana which lacks activation of RuBP carboxylase invivo. Plant Physiology, 1982. 70(2): p. 381-387.

111. Portis Jr, A.R. and M.E. Salvucci, The discovery of Rubisco activase - yet another story of serendipity. Photosynthesis Research, 2002. 73(1-3): p. 257-64.

112. Salvucci, M.E., et al., purification and species distribution of Rubisco activase. Plant Physiology, 1987. 84(3): p. 930-936.

113. Streusand, V.J. and A.R. Portis, Rubisco activase mediates ATP-dependent activation of ribulose bisphosphate carboxylase. Plant Physiology, 1987. 85(1): p. 152-154.

114. Mueller-Cajar, O., et al., Structure and function of the AAA(+) protein CbbX, a red-type Rubisco activase. Nature, 2011. 479(7372): p. 194-U66.

115. Tsai, Y.-C.C., et al., Identification and characterization of multiple rubisco activases in chemoautotrophic bacteria. Nature Communications, 2015. 6.

116. Neuwald, A.F., et al., AAA(+): A class of chaperone-like ATPases associated with the assembly, operation, and disassembly of protein complexes. Genome Research, 1999. 9(1): p. $27-43$.

117. Hanson, P.I. and S.W. Whiteheart, AAA+ proteins: have engine, will work. Nature Reviews Molecular Cell Biology, 2005. 6(7): p. 519-29.

118. Vale, R.D., AAA proteins. Lords of the ring. Journal of Cell Biology, 2000. 150(1): p. F13-9.

119. Ogura, T. and A.J. Wilkinson, AAA+ superfamily ATPases: common structure-diverse function. Genes to Cells, 2001. 6(7): p. 575-597.

120. Erzberger, J.P. and J.M. Berger, Evolutionary relationships and structural mechanisms of $A A A+$ proteins. Annual Review of Biophysics and Biomolecular Structure, 2006. 35: p. 93114.

121. Wendler, P., et al., Structure and function of the AAA+ nucleotide binding pocket. Biochimica et Biophysica Acta, 2012. 1823(1): p. 2-14.

122. Ogura, T., S.W. Whiteheart, and A.J. Wilkinson, Conserved arginine residues implicated in ATP hydrolysis, nucleotide-sensing, and inter-subunit interactions in $A A A$ and $A A A+$ ATPases. Journal of Structural Biology, 2004. 146(1-2): p. 106-12.

123. Yamada-Inagawa, T., et al., Conserved pore residues in the AAA protease FtsH are important for proteolysis and its coupling to ATP hydrolysis. Journal of Biological Chemistry, 2003. 278(50): p. 50182-50187. 
124. Siddiqui, S.M., R.T. Sauer, and T.A. Baker, Role of the processing pore of the ClpX AAA+ ATPase in the recognition and engagement of specific protein substrates. Genes \& Development, 2004. 18(4): p. 369-374.

125. Martin, A., T.A. Baker, and R.T. Sauer, Pore loops of the AAA+ClpX machine grip substrates to drive translocation and unfolding. Nature Structural \& Molecular Biology, 2008a. 15(11): p. 1147-1151.

126. Davies, J.M., A.T. Brunger, and W.I. Weis, Improved structures of full-length p97, an AAA ATPase: implications for mechanisms of nucleotide-dependent conformational change. Structure, 2008. 16(5): p. 715-26.

127. Wang, F., et al., Structure and mechanism of the hexameric MecA-ClpC molecular machine. Nature, 2011. 471(7338): p. 331-335.

128. Roll-Mecak, A. and R.D. Vale, Structural basis of microtubule severing by the hereditary spastic paraplegia protein spastin. Nature, 2008. 451(7176): p. 363-7.

129. Stotz, M., et al., Structure of green-type Rubisco activase from tobacco. Nature Structural \& Molecular Biology, 2011. 18(12): p. 1366-U78.

130. Henderson, J.N., et al., Atomic resolution X-ray structure of the substrate recognition domain of higher plant ribulose-bisphosphate carboxylaseloxygenase (Rubisco) activase. Journal of Biological Chemistry, 2011. 286(41): p. 35683-35688.

131. Hasse, D., A.M. Larsson, and I. Andersson, Structure of Arabidopsis thaliana Rubisco activase. Acta Crystallographica Section D: Biological Crystallography, 2015. 71(4): p. 800808.

132. Li, C., M.E. Salvucci, and A.R. Portis, Jr., Two residues of rubisco activase involved in recognition of the Rubisco substrate. Journal of Biological Chemistry, 2005. 280(26): p. 24864-9.

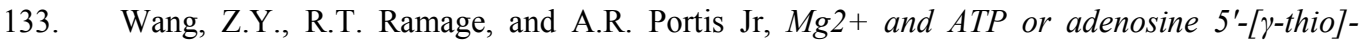
triphosphate $(A T P \gamma S)$ enhances intrinsic fluorescence and induces aggregation which increases the activity of spinach Rubisco activase. Biochimica et Biophysica Acta (BBA) Protein Structure and Molecular Enzymology, 1993. 1202(1): p. 47-55.

134. Barta, C., et al., Structural changes associated with the acute thermal instability of Rubisco activase. Archives of Biochemistry and Biophysics, 2010. 499(1-2): p. 17-25.

135. Keown, J.R., et al., Small oligomers of ribulose-bisphosphate carboxylaseloxygenase (Rubisco) activase are required for biological activity. Journal of Biological Chemistry, 2013. 288(28): p. 20607-15.

136. Salvucci, M.E., Subunit interactions of Rubisco activase: polyethylene glycol promotes selfassociation, stimulates ATPase and activation activities, and enhances interactions with Rubisco. Archives of Biochemistry and Biophysics, 1992. 298(2): p. 688-96.

137. Chakraborty, M., et al., Protein oligomerization monitored by fluorescence fluctuation spectroscopy: self-assembly of rubisco activase. Biophysical Journal 2012. 103(5): p. 949-58.

138. Henderson, J.N., et al., Biophysical characterization of higher plant Rubisco activase. Biochimica et Biophysica Acta, 2013. 1834(1): p. 87-97.

139. Buchen-Osmond, C., A. Portis, and J. Andrews. Rubisco activase modifies the appearance of rubisco in the electron microscope. in Research in photosynthesis: proceedings of the IXth International Congres. 1992.

140. Blayney, M.J., S.M. Whitney, and J.L. Beck, NanoESI mass spectrometry of Rubisco and Rubisco activase structures and their interactions with nucleotides and sugar phosphates. Journal of the American Society for Mass Spectrometry, 2011. 22(9): p. 1588-601. 
141. Keown, J.R. and F.G. Pearce, Characterization of spinach Ribulose-1, 5-bisphosphate carboxylase/oxygenase activase isoforms reveals hexameric assemblies with increased thermal stability. Biochemical Journal, 2014. 464(3): p. 413-423.

142. Werneke, J.M., R.E. Zielinski, and W.L. Ogren, Structure and expression of spinach leaf cDNA encoding ribulosebisphosphate carboxylase/oxygenase activase. Proceedings of the National Academy of Sciences of the United States of America, 1988. 85(3): p. 787-91.

143. Werneke, J.M., J.M. Chatfield, and W.L. Ogren, Alternative mRNA splicing generates the two ribulosebisphosphate carboxylase/oxygenase activase polypeptides in spinach and Arabidopsis. The Plant Cell, 1989. 1(8): p. 815-25.

144. To, K.Y., D.F. Suen, and S.C.G. Chen, Molecular characterization of ribulose-1,5bisphosphate carboxylase/oxygenase activase in rice leaves. Planta, 1999. 209(1): p. 66-76.

145. Portis, A.R., Jr., et al., Regulation of Rubisco activase and its interaction with Rubisco. Journal of Experimental Botany, 2008. 59(7): p. 1597-604.

146. Zhang, N. and A.R. Portis, Mechanism of light regulation of Rubisco: A specific role for the larger Rubisco activase isoform involving reductive activation by thioredoxin-f. Proceedings of the National Academy of Sciences of the United States of America, 1999. 96(16): p. 94389443.

147. Salvucci, M.E., F.J. van de Loo, and D. Stecher, Two isoforms of Rubisco activase in cotton, the products of separate genes not alternative splicing. Planta, 2003. 216(5): p. 736-44.

148. Shen, J.B., E.M. Orozco, and W.L. Ogren, Expression of the two isoforms of spinach ribulose 1,5-bisphosphate carboxylase activase and essentiality of the conserved lysine in the consensus nucleotide-binding domain. Journal of Biological Chemistry, 1991. 266(14): p. 8963-8968.

149. Schurmann, P. and J.P. Jacquot, Plant thioredoxin systems revisited. Annual Review of Plant Physiology and Plant Molecular Biology, 2000. 51: p. 371-400.

150. Balmer, Y. and B.B. Buchanan, Yet another plant thioredoxin. Trends in Plant Science, 2002. 7(5): p. 191-3.

151. Dai, S., et al., Structural snapshots along the reaction pathway of ferredoxin-thioredoxin reductase. Nature, 2007. 448(7149): p. 92-6.

152. Zhang, N., et al., Light modulation of Rubisco in Arabidopsis requires a capacity for redox regulation of the larger Rubisco activase isoform. Proceedings of the National Academy of Sciences of the United States of America, 2002. 99(5): p. 3330-4.

153. Taiz, L. and E. Zeiger, Plant Physiology. 2002: Sinauer Associates.

154. Carmo-Silva, A.E. and M.E. Salvucci, The regulatory properties of rubisco activase differ among species and affect photosynthetic induction during light transitions. Plant Physiology, 2013. 161(4): p. 1645-1655.

155. Zhang, N., P. Schürmann, and A. Portis, Jr., Characterization of the regulatory function of the 46-kDa isoform of Rubisco activase from Arabidopsis. Photosynthesis Research, 2001. 68(1): p. 29-37.

156. Wang, D. and A.R. Portis, Jr., Increased sensitivity of oxidized large isoform of ribulose-1,5bisphosphate carboxylase/oxygenase (rubisco) activase to ADP inhibition is due to an interaction between its carboxyl extension and nucleotide-binding pocket. Journal of Biological Chemistry, 2006. 281(35): p. 25241-9.

157. Rundle, S.J. and R.E. Zielinski, Alterations in barley ribulose-1,5-bisphosphate carboxylase oxygenase activase gene-expression during development and in response to illumination. Journal of Biological Chemistry, 1991. 266(22): p. 14802-14807. 
158. Stotz, M., Structure of green type Rubisco activase from Nicotiana tabacum (PhD thesis). Retreived from https://edoc.ub.uni-muenchen.de/14494/1/Stotz_Mathias_M.pdf, 2012.

159. van de Loo, F.J. and M.E. Salvucci, Activation of ribulose-1,5-biphosphate carboxylase/oxygenase (Rubisco) involves Rubisco activase Trp16. Biochemistry, 1996. 35(25): p. $8143-8$.

160. Esau, B.D., G.W. Snyder, and A.R. Portis, Differential effects of N-and C-terminal deletions on the two activities of rubisco activase. Archives of Biochemistry and Biophysics, 1996. 326(1): p. 100-105.

161. Salvucci, M.E., et al., Photoaffinity labeling of ribulose-1,5-bisphosphate carboxylase/oxygenase activase with ATP gamma-benzophenone. Identification of the ATP gamma-phosphate binding domain. Journal of Biological Chemistry, 1993. 268(19): p. 1423944.

162. Shen, J.B. and W.L. Ogren, Alteration of spinach ribulose-1,5-bisphosphate carboxylase oxygenase activase activities by site-directed mutagenesis. Plant Physiology, 1992. 99(3): p. 1201-1207.

163. van de Loo, F.J. and M.E. Salvucci, Involvement of two aspartate residues of Rubisco activase in coordination of the ATP gamma-phosphate and subunit cooperativity. Biochemistry, 1998. 37(13): p. 4621-5

164. Salvucci, M.E. and R.R. Klein, Site-directed mutagenesis of a reactive lysyl residue (Lys-247) of Rubisco activase. Archives of Biochemistry and Biophysics, 1994. 314(1): p. 178-85.

165. Li, C.S., D.F. Wang, and A.R. Portis, Identification of critical arginine residues in the functioning of Rubisco activase. Archives of Biochemistry and Biophysics, 2006. 450(2): p. 176-182.

166. Kallis, R.P., R.G. Ewy, and A.R. Portis, Alteration of the adenine nucleotide response and increased Rubisco activation activity of Arabidopsis Rubisco activase by site-directed mutagenesis. Plant Physiology, 2000. 123(3): p. 1077-1086.

167. Larson, E.M., et al., Specificity for activase is changed by a Pro-89 to Arg substitution in the large subunit of ribulose-1,5-bisphosphate carboxylase/oxygenase. Journal of Biological Chemistry, 1997. 272(27): p. 17033-17037.

168. Ott, C.M., et al., Activase region on chloroplast ribulose-1,5-bisphosphate carboxylase/oxygenase - Nonconservative substitution in the large subunit alters species specificity of protein interaction. Journal of Biological Chemistry, 2000. 275(34): p. 2624126244.

169. Salvucci, M.E. and S.J. Crafts-Brandner, Relationship between the heat tolerance of photosynthesis and the thermal stability of rubisco activase in plants from contrasting thermal environments. Plant Physiology, 2004. 134(4): p. 1460-70.

170. Cen, Y.-P. and R.F. Sage, The Regulation of Rubisco activity in response to variation in temperature and atmospheric $\mathrm{CO}_{2}$ partial pressure in sweet potato. Plant Physiology, 2005. 139(2): p. 979-990.

171. Berry, a. and O. Bjorkman, Photosynthetic response and adaptation to temperature in higher plants. Annual Review of Plant Physiology, 1980. 31(1): p. 491-543.

172. Law, R.D. and S.J. Crafts-Brandner, Inhibition and acclimation of photosynthesis to heat stress is closely correlated with activation of ribulose-1,5-bisphosphate carboxylase/oxygenase. Plant Physiology, 1999. 120(1): p. 173-182.

173. Havaux, M. and F. Tardy, Temperature-dependent adjustment of the thermal stability of photosystem II in vivo: possible involvement of xanthophyll-cycle pigments. Planta, 1996. 198(3): p. 324-333. 
174. Yamori, W., K. Hikosaka, and D.A. Way, Temperature response of photosynthesis in C3, C4, and CAM plants: temperature acclimation and temperature adaptation. Photosynthesis Research, 2014. 119(1): p. 101-117.

175. Wise, R.R., et al., Electron transport is the functional limitation of photosynthesis in fieldgrown Pima cotton plants at high temperature. Plant, Cell \& Environment, 2004. 27(6): p. 717-724.

176. Jordan, D.B. and W.L. Ogren, The $\mathrm{CO}_{2} / \mathrm{O}_{2}$ specificity of ribulose 1,5-bisphosphate carboxylase/oxygenase. Planta, 1984. 161(4): p. 308-313.

177. Scafaro, A.P., et al., Heat tolerance in a wild Oryza species is attributed to maintenance of Rubisco activation by a thermally stable Rubisco activase ortholog. New Phytologist, 2016. 211(3): p. 899-911.

178. Crafts-Brandner, S.J., F.J. Van De Loo, and M.E. Salvucci, The two forms of ribulose-1,5bisphosphate carboxylase/oxygenase activase differ in sensitivity to elevated temperature. Plant Physiology, 1997. 114(2): p. 439-444.

179. Sambrook, J. and D.W. Russell, Molecular Cloning: A Laboratory Manual. 2001: Cold Spring Harbor Laboratory Press.

180. Catanzariti, A.M., et al., An efficient system for high-level expression and easy purification of authentic recombinant proteins. Protein Science, 2004. 13(5): p. 1331-1339.

181. Baker, R.T., et al., Using Deubiquitylating Enzymes as Research Tools. Methods in Enzymology, 2005. 398: p. 540-554.

182. Gross, S.M., et al., De novo transcriptome assembly of drought tolerant CAM plants, Agave deserti and Agave tequilana. BMC Genomics, 2013. 14: p. 563.

183. Corpet, F., Multiple sequence alignment with hierarchical-clustering. Nucleic Acids Research, 1988. 16(22): p. 10881-10890.

184. Gouet, P., et al., ESPript: analysis of multiple sequence alignments in PostScript. Bioinformatics, 1999. 15(4): p. 305-308.

185. DeLano, W.L., The PyMOL molecular graphics system. 2002.

186. Gasteiger, E., et al., Protein identification and analysis tools on the ExPASy server. 2005: Springer.

187. Laemmli, U.K., Cleavage of structural proteins during assembly of head of bacteriophage-T4. Nature, 1970. 227(5259): p. 680-\&.

188. Makino, A., T. Mae, and K. Ohira, Purification and storage of ribulose 1,5-bisphosphate carboxylase from rice leaves. Plant and Cell Physiology, 1983. 24(6): p. 1169-1173.

189. Kreuzer, K.N. and C.V. Jongeneel, Escherichia coli phage T4 topoisomerase. Methods in enzymology, 1983. 100: p. 144-60.

190. Kubien, D.S., C.M. Brown, and H.J. Kane, Quantifying the amount and activity of Rubisco in leaves. Methods in Molecular Biology, 2011. 684: p. 349-62.

191. Horecker, B.L., J. Hurwitz, and A. Weissbach, Ribulose diphosphate. Biochem. Prep, 1958. 6: p. 83-90.

192. Kane, H.J., et al., Potent inhibition of ribulose-bisphosphate carboxylase by an oxidized impurity in ribulose-1,5-bisphosphate. Plant physiology, 1998. 117(3): p. 1059-1069.

193. DeSantis, M.E., et al., Operational plasticity enables Hsp104 to disaggregate diverse amyloid and nonamyloid clients. Cell, 2012. 151(4): p. 778-93. 
194. Werbeck, N.D., S. Schlee, and J. Reinstein, Coupling and dynamics of subunits in the hexameric AAA+ chaperone ClpB. J Mol Biol, 2008. 378(1): p. 178-90.

195. Jackrel, M.E., et al., Potentiated Hsp104 variants antagonize diverse proteotoxic misfolding events. Cell, 2014. 156(1-2): p. 170-82.

196. Wang, D., et al., Two Rubisco activase isoforms may play different roles in photosynthetic heat acclimation in the rice plant. Physiologia Plantarum, 2010. 139(1): p. 55-67.

197. Scafaro, A.P., P.A. Haynes, and B.J. Atwell, Physiological and molecular changes in Oryza meridionalis Ng., a heat-tolerant species of wild rice. Journal of Experimental Botany, 2010. 61(1): p. 191-202.

198. Lan, Y. and K.A. Mott, Determination of apparent Km values for ribulose 1,5-bisphosphate carboxylase oxygenase (Rubisco) activase using the spectrophotometric assay of Rubisco activity. Plant Physiology, 1991. 95(2): p. 604-609.

199. Schurmann, P. and B.B. Buchanan, The ferredoxin/thioredoxin system of oxygenic photosynthesis. Antioxid Redox Signal, 2008. 10(7): p. 1235-74.

200. Dai, S., et al., How does light regulate chloroplast enzymes? Structure-function studies of the ferredoxin/thioredoxin system. Quarterly Reviews of Biophysics, 2000. 33(1): p. 67-108.

201. Holmgren, A., Thioredoxin structure and mechanism - Conformational-changes on oxidation of the active-site sulfhydryls to a disulfide. Structure, 1995. 3(3): p. 239-243.

202. Brandes, H.K., et al., Direct identification of the primary nucleophile of thioredoxin-f. Journal of Biological Chemistry, 1993. 268(25): p. 18411-18414.

203. Goyer, A., et al., The internal Cys-207 of sorghum leaf NADP-malate dehydrogenase can form mixed disulphides with thioredoxin. FEBS Letters, 1999. 444(2-3): p. 165-169.

204. Balmer, Y. and P. Schurmann, Heterodimer formation between thioredoxin $f$ and fructose 1,6bisphosphatase from spinach chloroplasts. FEBS Letters, 2001. 492(1-2): p. 58-+.

205. Sanz-Barrio, R., et al., Chaperone-like properties of tobacco plastid thioredoxins $f$ and $\mathrm{m}$. Journal of Experimental Botany, 2012. 63(1): p. 365-379.

206. del Val, G., et al., Modification of the reactivity of spinach chloroplast thioredoxin $f$ by sitedirected mutagenesis. Plant Science, 1999. 149(2): p. 183-190.

207. Soulie, J.-M., et al., Molecular properties of chloroplastic thioredoxin $f$ and the photoregulation of the activity of fructose 1,6-bisphosphatase. European Journal of Biochemistry, 1981. 119(3): p. 497-502.

208. Capitani, G., et al., Crystal structures of two functionally different thioredoxins in spinach chloroplasts1. Journal of Molecular Biology, 2000. 302(1): p. 135-154.

209. Soulie, J.-M., et al., Equilibrium binding of thioredoxin FB to chloroplastic fructose bisphosphatase. European Journal of Biochemistry, 1985. 152(3): p. 565-568.

210. Nuruzzaman, M., et al., Sequence and expression analysis of the thioredoxin protein gene family in rice. Molecular Genetics and Genomics, 2008. 280(2): p. 139.

211. Collin, V., et al., The Arabidopsis plastidial thioredoxins: new functions and new insights into specificity. J Biol Chem, 2003. 278(26): p. 23747-52.

212. DeRidder, B.P., et al., Changes at the 3 '-untranslated region stabilize Rubisco activase transcript levels during heat stress in Arabidopsis. Planta, 2012. 236(2): p. 463-476.

213. Greenfield, N.J., Using circular dichroism spectra to estimate protein secondary structure. Nat Protoc, 2006. 1(6): p. 2876-90. 
214. Perez-Iratxeta, C. and M.A. Andrade-Navarro, K2D2: Estimation of protein secondary structure from circular dichroism spectra. BMC Structural Biology, 2008. 8: p. 25-25.

215. Salvucci, M.E., et al., Exceptional sensitivity of Rubisco activase to thermal denaturation in vitro and in vivo. Plant Physiology, 2001. 127(3): p. 1053-64.

216. Feller, U., S.J. Crafts-Brandner, and M.E. Salvucci, moderately high temperatures inhibit ribulose-1,5-bisphosphate carboxylase/oxygenase (Rubisco) activase-mediated activation of Rubisco. Plant Physiology, 1998. 116(2): p. 539-46.

217. Ades, S.E., AAA+ molecular machines: firing on all cylinders. Current Biology, 2006. 16(2): p. R46-8.

218. IPCC, Climate Change 2007: The physical science basis. Contribution of working group I to the fourth assessment report of the intergovernmental panel on climate change, S. Solomon, et al., Editors. 2007: United Kingdom and New York, NY, USA: Cambridge University Press, Cambridge.

219. Lobell, D.B. and G.P. Asner, Climate and management contributions to recent trends in US agricultural yields. Science, 2003. 299(5609): p. 1032-1032.

220. Carmo-Silva, A.E. and M.E. Salvucci, The temperature response of $\mathrm{CO}_{2}$ assimilation, photochemical activities and Rubisco activation in Camelina sativa, a potential bioenergy crop with limited capacity for acclimation to heat stress. Planta, 2012. 236(5): p. 1433-45.

221. Carmo-Silva, A.E., et al., Decreased $\mathrm{CO}_{2}$ availability and inactivation of Rubisco limit photosynthesis in cotton plants under heat and drought stress in the field. Environmental and Experimental Botany, 2012. 83: p. 1-11.

222. Sage, R.F., Photorespiratory compensation: a driver for biological diversity. Plant Biology, 2013. 15(4): p. 624-638.

223. Borland, A.M., et al., Exploiting the potential of plants with crassulacean acid metabolism for bioenergy production on marginal lands. Journal of Experimental Botany, 2009. 60(10): p. 2879-96.

224. Lüttge, U., Ecophysiology of crassulacean acid metabolism (CAM). Annals of Botany, 2004. 93(6): p. 629-652.

225. Brandon, P.C., Temperature features of enzymes affecting crassulacean acid metabolism. Plant Physiology, 1967. 42(7): p. 977-984.

226. Wang, Z.-Y., et al., Species-dependent variation in the interaction of substrate-bound ribulose-1,5-bisphosphate carboxylase/oxygenase (Rubisco) and Rubisco activase. Plant Physiology, 1992. 100(4): p. 1858-1862.

227. Ellis, R.J., Macromolecular crowding: obvious but underappreciated. Trends in Biochemical Sciences, 2001. 26(10): p. 597-604.

228. Zavodszky, P., et al., Adjustment of conformational flexibility is a key event in the thermal adaptation of proteins. Proceedings of the National Academy of Sciences of the United States of America, 1998. 95(13): p. 7406-7411.

229. Carmo-Silva, A.E. and M.E. Salvucci, The activity of Rubisco's molecular chaperone, Rubisco activase, in leaf extracts. Photosynthesis Research, 2011. 108(2-3): p. 143-55.

230. Yamori, W., et al., Rubisco activase is a key regulator of non-steady-state photosynthesis at any leaf temperature and, to a lesser extent, of steady-state photosynthesis at high temperature. Plant Journal, 2012. 71(6): p. 871-880. 
231. Esau, B.D., G.W. Snyder, and A.R. Portis Jr, Activation of ribulose-1, 5-bisphosphate carboxylase/oxygenase (Rubisco) with chimeric activase proteins. Photosynthesis Research, 1998. 58(2): p. 175-181.

232. Crooks, G.E., et al., WebLogo: A sequence logo generator. Genome Research, 2004. 14(6): p. 1188-1190.

233. Crayn, D.M., K. Winter, and J.A.C. Smith, Multiple origins of crassulacean acid metabolism and the epiphytic habit in the Neotropical family Bromeliaceae. Proceedings of the National Academy of Sciences of the United States of America, 2004. 101(10): p. 3703-3708.

234. Lüttge, U., Ability of crassulacean acid metabolism plants to overcome interacting stresses in tropical environments. AoB Plants, 2010. 2010: p. plq005.

235. Joly, N., N. Zhang, and M. Buck, ATPase site architecture is required for self-assembly and remodeling activity of a hexameric AAA plus transcriptional activator. Molecular Cell, 2012. 47(3): p. 484-490.

236. Moreau, M.J., et al., ATPase site architecture and helicase mechanism of an archaeal MCM. Molecular Cell, 2007. 28(2): p. 304-14.

237. Hersch, G.L., et al., Asymmetric interactions of ATP with the AAA+ClpX 6 unfoldase: allosteric control of a protein machine. Cell, 2005. 121(7): p. 1017-1027.

238. Martin, A., T.A. Baker, and R.T. Sauer, Rebuilt AAA + motors reveal operating principles for ATP-fuelled machines. Nature, 2005. 437(7062): p. 1115-20.

239. Weibezahn, J., et al., Characterization of a trap mutant of the AAA+ chaperone ClpB. Journal of Biological Chemistry, 2003. 278(35): p. 32608-17.

240. Kummer, E., et al., Bacterial and yeast AAA+ disaggregases ClpB and Hsp104 operate through conserved mechanism involving cooperation with Hsp70. Journal of Molecular Biology, 2016. 428(21): p. 4378-4391.

241. Hoskins, J.R., S.M. Doyle, and S. Wickner, Coupling ATP utilization to protein remodeling by $C l p B$, a hexameric $A A A+$ protein. Proceedings of the National Academy of Sciences of the United States of America, 2009. 106(52): p. 22233-22238.

242. Djuranovic, S., et al., Structure and activity of the N-terminal substrate recognition domains in proteasomal ATPases. Molecular Cell, 2009. 34(5): p. 580-590.

243. Scott, A., et al., Structure and ESCRT-III protein interactions of the MIT domain of human $V P S 4 A$. Proceedings of the National Academy of Sciences of the United States of America, 2005. 102(39): p. 13813-13818.

244. Sweeny, E.A., et al., The Hsp104 N-terminal domain enables disaggregase plasticity and potentiation. Molecular Cell, 2015. 57(5): p. 836-849.

245. Sauer, R.T., et al., Sculpting the proteome with AAA+ proteases and disassembly machines. Cell, 2004. 119(1): p. 9-18.

246. Wachter, R.M., et al., Activation of interspecies-hybrid Rubisco enzymes to assess different models for the Rubisco-Rubisco activase interaction. Photosynthesis Research, 2013. 117(13): p. 557-66.

247. Lavoie, C., et al., Tyrosine phosphorylation of p97 regulates transitional endoplasmic reticulum assembly in vitro. Proceedings of the National Academy of Sciences of the United States of America, 2000. 97(25): p. 13637-13642.

248. Whitehead, E., R. Heald, and J.D. Wilbur, N-terminal phosphorylation of p60 katanin directly regulates microtubule severing. Journal of Molecular Biology, 2013. 425(2): p. 214-221. 
249. Ewens, C.A., et al., Structural and functional implications of phosphorylation and acetylation in the regulation of the AAA+ protein $p 97$. Biochemistry and Cell Biology-Biochimie Et Biologie Cellulaire, 2010. 88(1): p. 41-48.

250. Boex-Fontvieille, E., et al., Phosphorylation pattern of Rubisco activase in Arabidopsis leaves. Plant Biology 2014. 16(3): p. 550-7.

251. Baginsky, S., Protein phosphorylation in chloroplasts - a survey of phosphorylation targets. Journal of Experimental Botany, 2016. 67(13): p. 3873-3882.

252. Kim, S.Y., et al., The plastid casein kinase 2 phosphorylates Rubisco activase at the Thr-78 site but is not essential for regulation of Rubisco activation state. Frontiers in Plant Science, 2016. 7.

253. Reiland, S., et al., Large-scale Arabidopsis phosphoproteome profiling reveals novel chloroplast kinase substrates and phosphorylation networks. Plant Physiology, 2009. 150(2): p. 889-903.

254. Peltier, J.-B., et al., New functions of the thylakoid membrane proteome of Arabidopsis thaliana revealed by a simple, fast, and versatile fractionation strategy. Journal of Biological Chemistry, 2004. 279(47): p. 49367-49383.

255. Sun, Q., et al., PPDB, the plant proteomics database at Cornell. Nucleic Acids Research, 2009. 37: p. D969-D974.

256. Winter, D., et al., An "Electronic fluorescent pictograph" browser for exploring and analyzing large-scale biological data sets. PLOS ONE, 2007. 2(8).

257. Hansen, B.O., et al., Elucidating gene function and function evolution through comparison of co-expression networks of plants. Frontiers in Plant Science, 2014. 5(394).

258. Ruprecht, C., et al., Large-scale co-expression approach to dissect secondary cell wall formation across plant species. Frontiers in Plant Science, 2011. 2(23).

259. Tamura, K., et al., MEGA6: molecular evolutionary genetics analysis version 6.0. Molecular Biology and Evolution, 2013: p. mst197.

260. Jiang, Y., et al., Characterization and expression of Rubisco activase genes in Ipomoea batatas. Molecular Biology Reports, 2013. 40(11): p. 6309-21.

261. NOAA, "NowData - NOAA Online Weather Data". National oceanic and atmospheric administration. 2016.

262. Fukayama, H., et al., Overexpression of Rubisco activase decreases the photosynthetic $\mathrm{CO}_{2}$ assimilation rate by reducing Rubisco content in rice leaves. Plant and Cell Physiology, 2012. 53(6): p. 976-986.

263. Sharkey, T.D., Some like it hot. Science, 2000. 287(5452): p. 435.

264. Yang, X.H., Z. Liang, and C.M. Lu, Genetic engineering of the biosynthesis of glycinebetaine enhances photosynthesis against high temperature stress in transgenic tobacco plants. Plant Physiology, 2005. 138(4): p. 2299-2309.

265. Feng, L.L., et al., Overexpression of SBPase enhances photosynthesis against high temperature stress in transgenic rice plants. Plant Cell Reports, 2007. 26(9): p. 1635-1646.

266. Murakami, Y., et al., Trienoic fatty acids and plant tolerance of high temperature. Science, 2000. 287(5452): p. 476-479.

267. Sauer, R.T. and T.A. Baker, AAA+ proteases: ATP-fueled machines of protein destruction. Annual Review of Biochemistry, 2011. 80: p. 587-612. 
268. Weibezahn, J., et al., Thermotolerance requires refolding of aggregated proteins by substrate translocation through the central pore of ClpB. Cell, 2004. 119(5): p. 653-665.

269. Scales, J.C., M.A. Parry, and M.E. Salvucci, A non-radioactive method for measuring Rubisco activase activity in the presence of variable ATP: ADP ratios, including modifications for measuring the activity and activation state of Rubisco. Photosynthesis Research, 2014. 119(3): p. 355-65.

270. Sutter, M., et al., Structural characterization of a newly identified component of $\alpha$ carboxysomes: The AAA+ domain protein CsoCbbQ. Scientific Reports, 2015. 5: p. 16243.

271. Andrews, T.J. and S.M. Whitney, Manipulating ribulose bisphosphate carboxylase/oxygenase in the chloroplasts of higher plants. Archives of Biochemistry and Biophysics, 2003. 414(2): p. 159-169.

272. Liu, C., et al., Coupled chaperone action in folding and assembly of hexadecameric Rubisco. Nature, 2010. 463(7278): p. 197-202.

273. Saschenbrecker, S., et al., Structure and function of RbcX, an assembly chaperone for hexadecameric Rubisco. Cell, 2007. 129(6): p. 1189-200.

274. Bracher, A., et al., Crystal structure of a chaperone-bound assembly intermediate of form I Rubisco. Nature Structural \& Molecular Biology, 2011. 18(8): p. 875-80.

275. Cloney, L.P., D.R. Bekkaoui, and S.M. Hemmingsen, Coexpression of plastid chaperonin genes and a synthetic plant rubisco operon in Escherichia coli. Plant Molecular Biology, 1993. 23(6): p. 1285-1290.

276. Mueller-Cajar, O. and S.M. Whitney, Directing the evolution of Rubisco and Rubisco activase: first impressions of a new tool for photosynthesis research. Photosynthesis Research, 2008. 98(1-3): p. 667-675.

277. Ochoa, D. and F. Pazos, Studying the co-evolution of protein families with the Mirrortree web server. Bioinformatics, 2010. 26(10): p. 1370-1.

278. Ovchinnikov, S., H. Kamisetty, and D. Baker, Robust and accurate prediction of residueresidue interactions across protein interfaces using evolutionary information. eLife, 2014. 3 : p. $\mathrm{e} 02030$.

279. de Juan, D., F. Pazos, and A. Valencia, Emerging methods in protein co-evolution. Nature Reviews Genetics, 2013. 14(4): p. 249-261.

280. Kromdijk, J., et al., Improving photosynthesis and crop productivity by accelerating recovery from photoprotection. Science, 2016. 354(6314): p. 857-861.

281. Lin, M.T., et al., A faster Rubisco with potential to increase photosynthesis in crops. Nature, 2014. 513(7519): p. 547-550.

282. Schwander, T., et al., A synthetic pathway for the fixation of carbon dioxide in vitro. Science, 2016. 354(6314): p. 900. 